\title{
Soft Landing of Size Selected Nanoparticles Produced by Magnetron Sputtering
}

\author{
Dissertation \\ for the award of the degree \\ of "Doctor of Philosophy" (Ph.D.) \\ Division of Mathematics and Natural Sciences \\ of Georg-August-Universität Göttingen
}

submitted by

Christopher Scott Larson

from the United States of America

Göttingen 2012 
D7

Referent: Alec Wodtke

Korreferent: Dirk Schwarzer

Date of Defense: November 23, 2012 


\section{Contents}

Foreword 3

I Photofragment Translational Spectroscopy of Methyl Azide 5

1 Introduction $\quad 7$

2 Previous Work and Literature Review 11

2.1 First Discovery of Azides and Structural Prediction . . . . . . . . . . . . . 11

2.2 First Experimental Evidence of Cyclic Isomer . . . . . . . . . . . . . . . 11

2.3 Theoretical Studies of Cyclic Isomer . . . . . . . . . . . . . . . . . 13

2.4 More Experimental Observations . . . . . . . . . . . . . . . . . . . 14

2.4.1 Ring Closing Dynamics of Hydrazoic Acid . . . . . . . . . . . . . . 14

2.4.2 Photofragment Translational Spectroscopy . . . . . . . . . . . . . . 14

2.4.3 More Velocity Map Imaging Experiments . . . . . . . . . . . . . . . 16

2.4.4 Exclusive Production of Cyclic Isomer . . . . . . . . . . . . . 16

3 Fundamentals 19

3.1 Photofragment Translational Spectroscopy . . . . . . . . . . . . . . . . 19

3.1 .1 Important Concepts . . . . . . . . . . . . . . . . . . . . . . . 19

3.1 .2 Typical Experimental Procedure . . . . . . . . . . . . . . . 22

3.2 Synchrotron Radiation . . . . . . . . . . . . . . . . . . . . . . . . . . . . . . . . . . . . . .

3.2.1 Theoretical Description and Discovery . . . . . . . . . . . . 23

3.2.2 Essential Components of a Synchrotron . . . . . . . . . . . . . . 24

3.2.3 Advantages of Synchrotron Light Sources . . . . . . . . . . . . . . . 25

4 Methods $\quad 27$

4.1 Synthesis and Characterization of Samples . . . . . . . . . . . . . . . . 27

4.2 Experimental Instrumentation . . . . . . . . . . . . . . . . . . . . . . . . . . . . . . . . . . . . . . . . .

4.3 Data Analysis . . . . . . . . . . . . . . . . . . . . . . . . . . . . . . . . . . . . . . . . . . . . .

4.3 .1 Pre-Processing . . . . . . . . . . . . . . . . 30

4.3.2 Principles of Forward Convolution Fitting . . . . . . . . . . . . . . 30

4.3.3 Computer Software for Forward Convolution Fitting . . . . . . . . . 31

5 Results and Discussion 33

5.1 Possible Dissociation Pathways . . . . . . . . . . . . . . . 33 
Contents

5.1.1 Primary Dissociation Pathways . . . . . . . . . . . . . . . 33

5.1 .2 Secondary Dissociation Pathways . . . . . . . . . . . . . . . . 35

$5.2248 \mathrm{~nm}$ Photolysis of Methyl Azide . . . . . . . . . . . . . . . . . . . . . . . . . . . . . . . . . .

5.2.1 Observed Photofragments . . . . . . . . . . . . 36

5.2 .2 Methyl Iodide Contamination . . . . . . . . . . . . . . 36

5.2.3 Overview of Thermodynamics of $248 \mathrm{~nm}$ Photolysis . . . . . . . . . . 38

5.2.4 Assignment of Primary Dissociation Channel . . . . . . . . . . . . . . . 38

5.2.5 Assignment of Secondary Dissociation Channels . . . . . . . . . . . . . 41

5.2 .6 Evidence of Singlet Methylnitrene . . . . . . . . . . . . . . . . 44

5.2.7 Comment on Role of Triplet Potential Energy Surface . . . . . . . . 45

$5.3193 \mathrm{~nm}$ Photolysis of Methyl Azide . . . . . . . . . . . . . . . . . 46

5.3.1 Observed Photofragments . . . . . . . . . . . . . 46

5.3.2 Overview of Thermodynamics of $193 \mathrm{~nm}$ Photolysis . . . . . . . . . . 48

5.3.3 Assignment of Primary Dissociation Channels . . . . . . . . . . . . 49

5.3.4 Assignment of Secondary Dissociation Channels . . . . . . . . . . 51

5.3 .5 Photoionization Threshold ................... 53

5.3.6 Evidence for Linear vs. Cyclic States of the Azide Radical . . . . . 54

5.3.7 Comment on Branching Ratio of Primary Dissociation Channels . . 56

6 Summary and Outlook 57

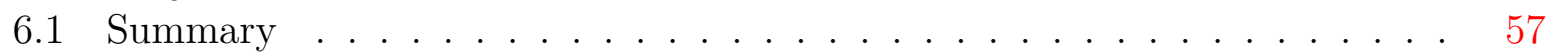

6.2 Further Work . . . . . . . . . . . . . . . . . . . . . . 59

6.3 Experimental Outlook ............................ 60

$\begin{array}{ll}\text { Bibliography } & 61\end{array}$

II Design and Construction of a Size Selected Nanoparticle Depo$\begin{array}{ll}\text { sition System } & 67\end{array}$

$\begin{array}{llr}7 & \text { Introduction } & 69\end{array}$

8 Fundamentals $\quad 71$

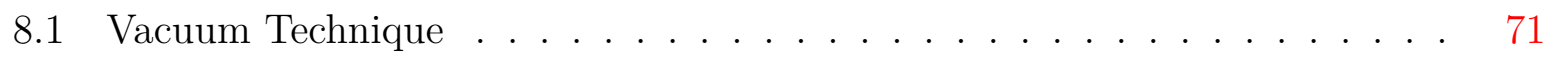

8.1 Mean Free Path . . . . . . . . . . . . . . . . . . . . 71

8.1 .2 Surface Coverage . . . . . . . . . . . . . . . . . . . . . . . . 72

8.1.3 Generating and Measuring Vacuum . . . . . . . . . . . . . . . . 73

8.1.4 Summary of Vacuum in Relation to Nanoparticle Deposition System 74

8.2 Magnetron Sputtering . . . . . . . . . . . . . . . . . 74

8.2.1 Development of the Magnetron Sputtering Source . . . . . . . . . . 74

8.2.2 Operating Principles . . . . . . . . . . . . . . . . . 75

8.2 .3 Cluster Growth Mechanisms . . . . . . . . . . . . . . . . 76

8.2 .4 Ion Formation Mechanisms . . . . . . . . . . . . . . . 76 
8.2.5 Shape of Mass Distribution . . . . . . . . . . . . . . . . 78

8.2.6 Calculating Nanoparticle Size from Mass . . . . . . . . . . . . . . . 78

8.2.7 Advantages and Disadvantages of Magnetron Sputtering . . . . . . 79

8.3 Quadrupole Mass Spectrometry . . . . . . . . . . . . . . . . . 80

8.3.1 Development of Quadrupole Mass Spectrometers . . . . . . . . . . . 80

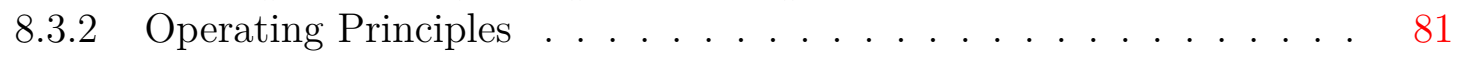

8.3.3 Mathieu's Equation and the Quadrupole Stability Region . . . . . . . 82

8.3.4 Equations of Transmitted Mass and Mass Resolution . . . . . . . . . 84

8.3.5 Use of Quadrupoles for Filtering High Masses . . . . . . . . . . . . 84

8.3.6 Advantages and Disadvantages of Mass Filtering with the QMF . . 85

8.4 Time of Flight Mass Spectrometry . . . . . . . . . . . . . . . . 85

8.4.1 Overview of Time of Flight Mass Spectrometry . . . . . . . . . . 86

8.4 .2 Fundamental Physics and Equations . . . . . . . . . . . . . 86

8.4.3 Further Time of Flight Techniques . . . . . . . . . . . . . . 88

8.5 Surface Analysis Techniques . . . . . . . . . . . . . . . . . . . . . . . . . . . . . . . . . . . . 88

8.5.1 Atomic Force Microscopy . . . . . . . . . . . . . . . . . . . . . 88

8.5.2 Transmission Electron Microscopy . . . . . . . . . . . . . . . 91

9 Experimental Setup and Instrumentation $\quad 95$

9.1 Instrument Overview . . . . . . . . . . . . . . . . . . . . . . . 95

9.2 Magnetron Sputtering Nanoparticle Source . . . . . . . . . . . . . . . . . . 96

9.3 Quadrupole Mass Filter . . . . . . . . . . . . . . . . . . . . . . . . . 98

9.4 Time of Flight Mass Spectrometer . . . . . . . . . . . . . . . . . . 100

9.5 Surface Deposition Chamber . . . . . . . . . . . . . 106

10 Characterization and Preliminary Results 111

10.1 Magnetron Sputtering Ion Source . . . . . . . . . . . . . . . . . . . . . . . 111

10.1.1 Trends in Cluster Size Distribution . . . . . . . . . . . . . . . 111

10.1 .2 Effect of Exit Aperture Size . . . . . . . . . . . . . . . . . 117

10.2 Time of Flight Mass Spectrometer . . . . . . . . . . . . . . . . . . . . . . . . . . . . . . .

10.2.1 Calibrating the Time-to-Mass Conversion . . . . . . . . . . . 118

10.2.2 Mass Resolution . . . . . . . . . . . . . . . . . . . . . . . . 122

10.2.3 Comparison of Experimental Results to Simulations . . . . . . . . . 123

10.3 Quadrupole Mass Filter . . . . . . . . . . . . . . . . 127

10.3.1 Quadrupole Mass Filter's Transmitted Mass Resolution . . . . . . . 127

10.3.2 Comparison of Time of Flight Results to Quadrupole Performance . 128

10.3.3 Calibration of the Quadrupole Mass Filter . . . . . . . . . . . . . 129

10.4 Characterization of Ion Beam . . . . . . . . . . . . . . . . . 131

10.5 Characterization of the Cluster Ion Energy . . . . . . . . . . . . . . . 132

10.5.1 Design and Construction of the Retarding Field Energy Analyzer . 132

10.5.2 Operation and Data Analysis . . . . . . . . . . . . . . 133

10.5.3 Ion Beam Kinetic Energy Measurements . . . . . . . . . . . . . . . 134

10.5.4 Model for Ion Beam Velocity . . . . . . . . . . . . . . . . . . . 139 
Contents

10.5.5 Considerations for Controlling Ion Energy . . . . . . . . . . . . . . 140

10.6 Microscopy of Surface Deposited Size Selected Clusters . . . . . . . . . . . 141

10.6.1 Atomic Force Microscopy . . . . . . . . . . . . . . . . . . . . . . . . 141

10.6.2 Transmission Electron Microscopy . . . . . . . . . . . . . . . . . . . 152

11 Summary and Outlook 163

11.1 Summary of Results . . . . . . . . . . . . . . . . . . . . . 163

11.2 Experimental Outlook . . . . . . . . . . . . . . 165

11.3 Outlook for Instrument Modifications and Improvements . . . . . . . . . . 167

$\begin{array}{ll}\text { Bibliography } & 169\end{array}$

$\begin{array}{lll}\text { Appendix A Quadrupole Mass Filter Calibration Data } & 173\end{array}$

Appendix B SIMION Geometry and Lua Program Files $\quad 175$

B.0.1 Geometry . . . . . . . . . . . . . . . . . 175

B.0.2 Lua Program . . . . . . . . . . . . . . . 178

$\begin{array}{ll}\text { Acknowledgements } & 181\end{array}$

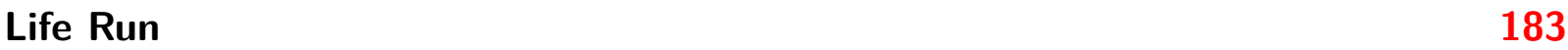




\section{List of Figures}

3.1 Newton diagram in photofragment translational spectroscopy . . . . . . . . 20

4.1 Instrument schematic drawing for azide experiment . . . . . . . . . . . . 29

5.1 Representative TOF spectra for all observed photofragments in $248 \mathrm{~nm}$ pho-

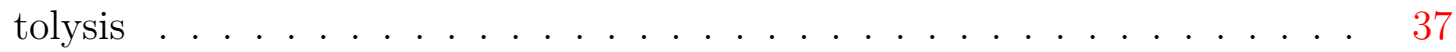

$5.2 \mathrm{~m} / z=14$ and 15 TOF spectra and forward convolution fitting for $248 \mathrm{~nm}$

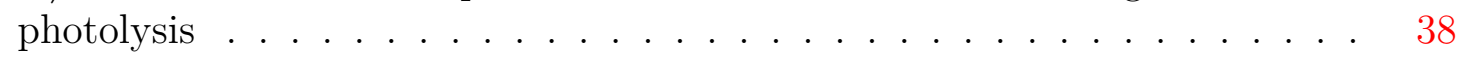

$5.3248 \mathrm{~nm}$ photolysis energy diagram . . . . . . . . . . . . . . . 39

$5.4 \mathrm{~m} / z=29$ and $28 \mathrm{TOF}$ spectra and forward convolution fitting for $248 \mathrm{~nm}$

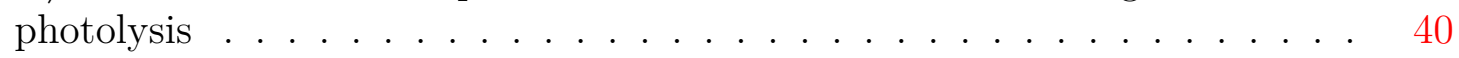

5.5 $\mathrm{P}\left(\mathrm{E}_{\mathrm{T}}\right)$ for primary dissociation channel in $248 \mathrm{~nm}$ photolysis . . . . . . . 41

$5.6 \mathrm{~m} / z=27$ TOF spectra and forward convolution fitting for $248 \mathrm{~nm}$ photolysis 42

$5.7 P\left(E_{\mathrm{T}}\right)$ for secondary dissociation channel in $248 \mathrm{~nm}$ photolysis . . . . . . 43

5.8 Low IE $m / z=28$ TOF spectra for $248 \mathrm{~nm}$ photolysis . . . . . . . . . . . . 44

5.9 Representative TOF spectra for all observed photofragments in $193 \mathrm{~nm}$ photolysis . . . . . . . . . . . . . . . . . . 47

$5.10 \mathrm{~m} / z=15$ TOF spectra with fit to $\mathrm{CH}_{3} \mathrm{I}$ contribution $\ldots \ldots \ldots . . . . \quad 48$

$5.11193 \mathrm{~nm}$ photolysis energy diagram . . . . . . . . . . . . . . . 49

$5.12 \mathrm{~m} / z=15$ and 42 TOF spectra and forward convolution fitting for $193 \mathrm{~nm}$

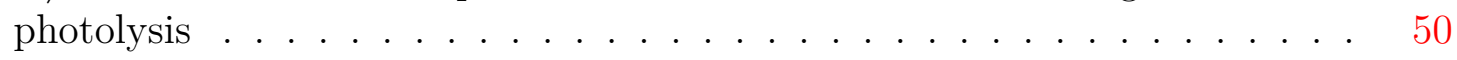

5.13 Translational energy release probability distributions for the radical dissociation channel in $193 \mathrm{~nm}$ photolysis . . . . . . . . . . . . . 51

$5.14 \mathrm{~m} / z=29$ TOF spectra and $P\left(E_{T}\right)$ for $193 \mathrm{~nm}$ photolysis . . . . . . . . 52

$5.15 \mathrm{~m} / z=14$ TOF spectra and $P\left(E_{T}\right)$ for $193 \mathrm{~nm}$ photolysis . . . . . . . 53

$5.16 \mathrm{~m} / z=28$ and 27 TOF spectra and forward convolution fitting for $193 \mathrm{~nm}$ photolysis ............................... 54

5.17 Photoionization threshold of $m / z=42$ product fragment . . . . . . . . 55

8.1 Magnetron head schematic diagram . . . . . . . . . . . . . . . . 75

8.2 Mathieu stability diagram . . . . . . . . . . . . . . . . . . . . . . . . . . . . . . . 83

8.3 First stability region of Mathieu diagram . . . . . . . . . . . . . . . . . 83

8.4 Time of flight drawing . . . . . . . . . . . . . . . . . . 86

8.5 Drawing of typical AFM . . . . . . . . . . . . . . . . . . . . . . . . . . . . . . . . .

8.6 Drawing of typical TEM . . . . . . . . . . . . . . . . 92 
List of Figures

9.1 Schematic diagram overview of the fully assembled size selected deposition

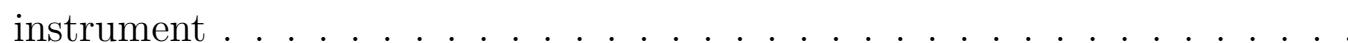

9.2 Schematic diagram overview of the time of flight analyzer configuration of the instrument . . . . . . . . . . . . . . . . 97

9.3 Schematic drawing of magnetron source chamber . . . . . . . . . . . . 98

9.4 Schematic drawing of quadrupole mass filter chamber . . . . . . . . . . . . . . 99

9.5 Schematic drawing of time of flight mass analyzer chamber . . . . . . . . . 101

9.6 Schematic of home built TOF analyzer . . . . . . . . . . . . . . . . . 102

9.7 Sample SIMION simulation screenshot . . . . . . . . . . . . . . . . 103

9.8 Simulated TOF resolution . . . . . . . . . . . . . . . . . . . 104

9.9 Schematic drawing of the surface deposition chamber . . . . . . . . . . . 107

9.10 Photograph of sample holder . . . . . . . . . . . . . . . . . . . 109

10.1 Effect of sputtering gas flow on cluster size distribution . . . . . . . . . . . 112

10.2 Effect of aggregation distance on cluster size distribution . . . . . . . . . . 114

10.3 Effect of sputtering power on cluster size distribution . . . . . . . . . . . 115

10.4 Effect of aggregation gas flow on cluster size distribution . . . . . . . . 116

10.5 Example of large and small size distributions . . . . . . . . . . . . . . . 117

$10.6 \mathrm{Ta}^{+}$cluster calibration of the TOF-to-mass conversion . . . . . . . . . . 119

10.7 Cluster mass vs TOF plot . . . . . . . . . . . . . . . . . . . . . 119

10.8 Experimental TOF mass resolution . . . . . . . . . . . . . . . . . . 122

10.9 Comparison of experimental TOF to simulation . . . . . . . . . . . 125

10.10 Effect of quadrupole resolution on mass distribution . . . . . . . . . . . . . . 127

10.11 Quadrupole mass offset . . . . . . . . . . . . . . . . 128

10.12 Quadrupole mass filter calibration plot . . . . . . . . . . . . . 130

10.13 Measured ion beam width . . . . . . . . . . . . . . . . . . . 131

10.14 Representative example of retarding field energy analyzer data . . . . . . 134

10.15 Effect of argon flow rate on cluster kinetic energy and velocity . . . . . . . 136

10.16 Ion beam energy distribution as function of quadrupole resolution . . . . . 137

10.17 Ion beam energy distribution as function of helium flow . . . . . . . . . . 138

10.18 Cluster velocity model . . . . . . . . . . . . . . . . . . . . 140

10.19 AFM image of 171,188 amu tantalum nanoparticles on silica substrate . . 145

10.20 AFM image of 237,000 amu tantalum nanoparticles on strontium titanate substrate . . . . . . . . . . . . . . . . 146

10.21 AFM image of 171,188 amu tantalum nanoparticles on mica substrate . . 147

10.22 AFM image of 49,129 amu tantalum nanoparticles on mica substrate . . . 148

10.23 AFM image of $61,628 \mathrm{amu}$ tantalum nanoparticles on strontium titanate substrate . . . . . . . . . . . . . . . . . . . . . 149

10.24 AFM image of 10\% Resolution 196,000 amu tantalum nanoparticles on mica substrate . . . . . . . . . . . . . . . . . 149

10.25 AFM image of $50 \%$ resolution 196,000 amu tantalum nanoparticles on mica substrate . . . . . . . . . . . . . . . . 150 
10.26 AFM images of 200,000 amu tantalum nanoparticle on mica in a temperature series . . . . . . . . . . . . . . . . . 151

10.27 TEM image of 200,000 amu tantalum nanoparticles . . . . . . . . . . 153 10.28 TEM histogram of 200,000 amu tantalum nanoparticles . . . . . . . . . . 154 10.29 TEM image of 800,000 amu tantalum nanoparticles . . . . . . . . . . . . . 155

10.30 TEM image of 50,000 amu tantalum nanoparticles . . . . . . . . . . . . 155

10.31 TEM image of 300,000 amu tantalum nanoparticles . . . . . . . . . . . . . 156

10.32 TEM histogram of 300,000 amu tantalum nanoparticles . . . . . . . . . 156

10.33 EDX point scans of tantalum nanoparticles . . . . . . . . . . . . . 159

10.34 EDX line scan of tantalum nanoparticles . . . . . . . . . . . . . . . . 160

10.35 TEM of 600,000 amu tantalum nanoparticle on graphene . . . . . . . . . . 161

10.36 High magnification TEM image of 600,000 amu tantalum nanoparticles on graphene . . . . . . . . . . . . . . . . . 161 
List of Figures 


\section{List of Tables}

8.1 Mean free path of $\mathrm{Ar}$ at $20^{\circ} \mathrm{C} \ldots \ldots \ldots \ldots$. . . . . . . . . . . . . . . . . . . . 72

8.2 Time to surface monolayer coverage for $\mathrm{H}_{2} \mathrm{O}$ at $20^{\circ}$ C . . . . . . . . 72

10.1 Calibration time of flight data. . . . . . . . . . . . . . . 120

10.2 Best fit parameters to experimental data. . . . . . . . . . . . . . . . . . . . . . . . . . . . . 121

10.3 Time-to-mass conversion equation coefficients. . . . . . . . . . . . . . 121

10.4 Comparison of experimental flight times to simulation. . . . . . . . . . . . 124

10.5 Comparison of experimental flight times to theoretically calculated flight times. . . . . . . . . . . . . . . . . . 126

10.6 Retarding field energy analyzer extracted fit for the effect of argon flow rate on cluster energy. . . . . . . . . . . . . . . . . . 135

10.7 Retarding field energy analyzer extracted fit for the effect of quadrupole mass resolution on cluster energy. . . . . . . . . . . . . . 138

10.8 Retarding field energy analyzer extracted fit for the effect of helium flow rate on cluster energy. . . . . . . . . . . . . . . . . . . . . . 139

10.9 Table of relevant X-ray energies adapted from Reference [53] . . . . . . . 158

A.1 Quadrupole mass filter calibration data. . . . . . . . . . . . 173 
List of Tables 


\section{Foreword}

A brief introduction regarding the structure of this dissertation and the time frame of experiments performed. My dissertation is divided into two parts which cover two distinct and separate research projects that I worked on over the course of my PhD work. The first project concerns a photofragment translational spectroscopy study of the organic molecule methyl azide, $\mathrm{CH}_{3} \mathrm{~N}_{3}$. This work was done at the beginning of my graduate studies at the University of California, Santa Barbara (UCSB) in 2005 and the results were published in two research papers in 2006 and 2008. As this work was completed several years ago, a colleague has continued this line of research and some of the newly understood information from his work will be presented in the conclusion chapter for this part. Following my work on methyl azide, I was presented with the opportunity to be involved in a new project to build an instrument for the size selected soft landing deposition of nanoparticles. The second part of my dissertation covers the design and characterization work I did for this instrument. In 2010, this project was moved from UCSB to Georg-August-Universität Göttingen as part of the Department of Dynamics at Surfaces, where I rebuilt the instrument and completed the characterization as the conclusion of my dissertation work. 


\section{Part I}

\section{Photofragment Translational Spectroscopy of Methyl Azide}





\section{Chapter 1}

\section{Introduction}

Homonuclear polynitrogen molecules, as the name suggests, are molecules composed solely of nitrogen atoms. Despite the large abundance of nitrogen in the universe, only a handful of homonuclear polynitrogen molecules are known to exist [53]. The first known polynitrogen molecule, $\mathrm{N}_{2}$, is also the only one which occurs naturally. The $\mathrm{N}_{2}$ molecule is extremely stable due to the strong triple bond between nitrogen atoms. Due to the strength and stability of this bond, many metastable homonuclear polynitrogen molecules are theoretically predicted to release large amounts of energy when they decompose into the more stable $\mathrm{N}_{2}$ molecules allowing for the storage of a large amount of energy per molecule. They belong to a class of compounds known as High Energy Density Materials (HEDM). Furthermore, since the only byproduct of decomposition of these molecules is $\mathrm{N}_{2}$, they are environmentally friendly. These properties make homonuclear polynitrogen molecules extremely interesting as a fuel source, since they contain a large amount of energy per molecule and leave no harmful byproducts.

Despite nitrogen's abundance and theoretical calculations showing that a number of these compounds are potentially stable, only a handful (including $\mathrm{N}_{2}$ ) have been experimentally proven to exist. There is a large barrier to producing these compounds due to the aforementioned stability of the $\mathrm{N}_{2}$ molecule.

Azides are a class of compounds containing three bonded nitrogen atoms. A number of collision-free studies of azide photochemistry have been performed in recent years [28-31, $52,55,56]$ and have provided new insights into the world of homonuclear polynitrogen chemistry. Two primary dissociation pathways from azide compound photolysis have been identified:

$$
\begin{aligned}
& \mathrm{X}-\mathrm{N}_{3} \stackrel{h \nu}{\longrightarrow} \mathrm{X}+\mathrm{N}_{3} \\
& \mathrm{X}-\mathrm{N}_{3} \stackrel{h \nu}{\longrightarrow} \mathrm{XN}+\mathrm{N}_{2}
\end{aligned}
$$

(Radical Channel)

(Molecular Elimination Channel)

In particular, the radical producing channel has been found to be more important than previously expected [31, 63]. A great deal of evidence has emerged that the $\mathrm{N}_{3}$ radical can appear in two different conformations - the well-known linear azide, and a recently discovered cyclic form: 
Chapter 1 Introduction

$$
\mathrm{N}=\mathrm{N}=\mathrm{N}^{-}
$$

Linear

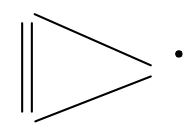

Cyclic

The cyclic form is of particular interest. Theoreticians have predicted the cyclic $\mathrm{N}_{3}$ radical to react with a $\mathrm{N}\left({ }^{2} \mathrm{D}\right)$ atom to produce tetraazatetrahedrane, a tetragonal configuration of $\mathrm{N}_{4}[6,68]$. Furthermore, tetragonal $\mathrm{N}_{4}$ is predicted to be metastable and a high energy density material. Understanding of the chemistry of the cyclic $\mathrm{N}_{3}$ radical can provide a new gateway into the world of homonuclear polynitrogen chemistry, the production of new stable nitrogen allotropes, and the creation of new high energy density materials.

Despite the rather compelling evidence for the existence of the cyclic $\mathrm{N}_{3}$ radical which has come to light, much about it is still not understood. There is a lack of reliable methods to produce large quantities of this conformation, making it difficult to examine with spectroscopic methods which could further elucidate its properties. With the goal in mind to advance the understanding of the properties of the cyclic $\mathrm{N}_{3}$ radical and the conditions by which it can be produced, I developed the experiment described in this thesis. The starting point for this experiment comes from the first experimental observations of cyclic $\mathrm{N}_{3}$ in the work of Nils Hansen et al. [30] where cyclic $\mathrm{N}_{3}$ was determined to be produced in the $193 \mathrm{~nm}$ photolysis of chlorine azide, $\mathrm{ClN}_{3}$. While the authors were able to detect cyclic $\mathrm{N}_{3}$ as a photofragment in this process, a large portion of the molecules had sufficient internal energy to undergo unimolecular dissociation into $\mathrm{N}+\mathrm{N}_{2}$. Several follow-up experiments to provide further evidence for the cyclic $\mathrm{N}_{3}$ isomer were performed by both Nils Hansen and Peter Samartzis [28, 29, 31, 52, 55, 56].

A photodissociation process leading to an internally cold cyclic $\mathrm{N}_{3}$ radical and a longer lifetime would allow for more detailed characterization in spectroscopic studies and use in further reactions. In order to improve the molecule's stability, photolytically producing the cyclic $\mathrm{N}_{3}$ radical with a lower amount of internal energy will reduce the likelihood of further unimolecular decomposition processes. Improvements in this area could be approached in two ways. First, the photolysis could be done using longer wavelengths with lower energy photons for dissociation. This would, in effect, lower the total amount of energy available in the dissociation, perhaps leading to less energetic distribution of product fragments. There are some fundamental limitations to taking this approach, however. There still needs to be enough energy in the photon to dissociate the parent molecule, which places a limit on how effective this method can be. Another troublesome point is that changing the absorption wavelength can completely change the photodissociation dynamics of the parent molecule, which may lead to no formation of the desired cyclic conformation. Another, more flexible approach, is to change the functional group bonded to the azide to one which has similar electronic structure, but both a stronger $\mathrm{X}-\mathrm{N}_{3}$ bond and making $\mathrm{X}$ a polyatomic functional group capable of carrying more energy in its internal degrees of freedom. With more of 
the photon energy going into breaking of the $\mathrm{X}-\mathrm{N}_{3}$ bond, less energy is available to excite the product fragments.

From these standpoints, methyl azide, $\mathrm{CH}_{3} \mathrm{~N}_{3}$, is a strong candidate for improving upon the previous chlorine azide work. The C-N bond energy in methyl azide is around $3.2 \mathrm{eV}$, which is almost twice as high as the $\mathrm{Cl}-\mathrm{N}$ bond in chlorine azide [26]. Furthermore, it moves from the atomic functional group in chlorine to a polyatomic functional group with the methyl group which has vibrational modes and more rotational degrees of freedom. If the electronic structure is similar enough, we may expect to see cyclic $\mathrm{N}_{3}$ produced under similar conditions with a lower internal energy and longer lifetimes than in the photolysis of $\mathrm{ClN}_{3}$.

With this in mind, I performed an experiment similar to the aforementioned ones on $\mathrm{ClN}_{3}$ performed by Hansen and Samartzis - a photofragment translational spectroscopic study of the collision-free photochemistry of methyl azide at $248 \mathrm{~nm}$ and $193 \mathrm{~nm}$ photolysis wavelengths. Photofragment Translational Spectroscopy (PTS) is a powerful technique, well-suited to examining the dissociation dynamics of a photolysis process. Expanding upon this line of work is a reasonable next step in the understanding of azide photochemistry.

The experimental work in this part of my dissertation will be presented as follows:

Chapter 2 will present a literature review and summary of the previous work leading up to this experiment, focusing on an overview of the most important theoretical and experimental papers and results which directly influence this dissertation work. In Chapter 3, an overview of the fundamental principles necessary to understanding the experimental apparatus and methods will be presented. Chapter 4 will cover details of the methods used including the synthesis of methyl azide samples, the experimental instrumentation, and the methods of analyzing data. The experimental results and discussion will be combined in Chapter 5 which is sub-divided into $248 \mathrm{~nm}$ photolysis and $193 \mathrm{~nm}$ photolysis sections. This division is meant to keep the two sets of results separate from each other, and the important data and figures related to each section fresh in the reader's mind during both the analysis and discussion. Finally, the conclusion in Chapter 6 summarizes the important results, presents ideas for potential future experiments which could derive from this work, and provides an overview of newly published experimental results by other researchers on this topic. 
Chapter 1 Introduction 


\section{Chapter 2}

\section{Previous Work and Literature Review}

This chapter provides the historical context leading to the conception of my dissertation work. It presents a review of relevant previous experiments, papers and results in a roughly chronological order of their occurrence. An excellent source of information on this topic has already been published in a review article on azide photochemistry by Peter Samartzis and Alec Wodtke [54]. I will cover many of the same topics and literature sources, but with the viewpoint centered on how those experiments built the foundations of this dissertation work. Brief overviews of experimental techniques needed to understand the significant milestones in cyclic $\mathrm{N}_{3}$ research will be presented as well when necessary.

\subsection{First Discovery of Azides and Structural Prediction}

The story of azide $\left(\mathrm{N}_{3}\right)$ research begins in 1864 when the first known azide containing molecule, phenyl azide, was synthesized by Peter Greiss [27]. Not much excitement was generated by this discovery at the time. In 1890, the simplest azide molecule, hydrazodic acid, $\mathrm{HN}_{3}$ was discovered by T. Curtius [17]. Curtius' paper presents the first guess as to the possible structure of an azide containing species. Interestingly, his only structural guesses were of cyclic complexes. The primary molecule of interest to this dissertation, methyl azide, $\mathrm{CH}_{3} \mathrm{~N}_{3}$ was first synthesized in 1905 by Otto Dimroth and Wilhelm Wislicenus [19].

Many years after the discovery of these azide compounds, when the first spectroscopic studies were finally performed, it became firmly established that $\mathrm{N}_{3}$ has a linear structure [20]. The evidence for a linear $\mathrm{N}_{3}$ structure was overwhelming, and Curtius' first speculations about a cyclic $\mathrm{N}_{3}$ structure were soon forgotten. It was not until decades later that the idea of a stable cyclic azide isomer began to take shape after a surreptitious observation of a high energy fragment in chlorine azide photodissociation which matched no known state of the linear azide radical.

\subsection{First Experimental Evidence of Cyclic Isomer}

This first experimental observation of what is likely a cyclic isomer of the $\mathrm{N}_{3}$ radical came in 2003 from a series of velocity map imaging (VMI) studies of the photolysis of $\mathrm{ClN}_{3}$ by Nils Hansen and Alec M. Wodtke [28-30]. 
A brief overview of the velocity map imaging technique is necessary to fully understand the significance of the observed results of this and other similar experiments. Velocity map imaging is a method for imaging which can be applied to photofragment ions in a photolysis process. A molecular beam of the molecule of interest is formed and subsequently photodissociated. The product fragments are ionized, often through some resonance enhanced multi photon ionization (REMPI) method. The ions can be focused onto a position sensitive detector with electrostatic lenses. These ion optics ensure that all ions with the same velocity are focused to the same radial position on the detector. The recorded image compiles the results of many thousands of photolysis events. The final captured image is a two dimensional projection of the three dimensional packet of dissociated ions. To reconstruct the three dimensional image, a technique known as the inverse Abel transform is used [21]. The final results provide information about the velocities of the recoiling fragments in the center of mass frame. Fragments with higher velocity move further from the center in the time it takes to reach the detector than low velocity fragments. The law of conservation of energy plays an important role in the analysis of these results. As the parent molecule has a fixed amount of energy available following the absorption of a single photon, detected product fragments with higher translational energy will produce a counterpart fragment with lower translational energy. This can directly lead to information about the internal states of the product fragments.

Returning to the experiments of Hansen et al., in the first paper $\mathrm{ClN}_{3}$ was photodissociated at near $203 \mathrm{~nm}$ dissociation wavelength and the resulting state-selected $\mathrm{N}_{2}\left(\mathrm{X}^{1} \Sigma_{g}^{+}\right.$, $\mathrm{v}=0, \mathrm{~J}=68)$ fragments were ionized with $2+1$ REMPI [30]. The experiment was able to clearly identify the state-selected $\mathrm{N}_{2}$ as coming from two separate dissociation channels:

$$
\begin{aligned}
& \mathrm{ClN}_{3} \stackrel{h \nu}{\longrightarrow} \mathrm{N}_{2}\left(\mathrm{X}^{1} \Sigma_{g}^{+}\right)+\mathrm{NCl}\left(\mathrm{a}^{1} \Delta\right) \\
& \mathrm{ClN}_{3} \stackrel{h \nu}{\longrightarrow} \mathrm{N}_{2}\left(\mathrm{X}^{1} \Sigma_{g}^{+}\right)+\mathrm{NCl}\left(\mathrm{X}^{3} \Sigma^{-}\right)
\end{aligned}
$$

$\mathrm{NCl}\left(\mathrm{a}^{1} \Delta\right)$ production was believed at the time to be the primary dissociation channel in $\mathrm{ClN}_{3}$ photodissociation [37] and this experiment seemed to confirm that belief by determining the branching ratio between these two channels to be 0.78 to 0.22 in favor of $\mathrm{NCl}$ $\left(a^{1} \Delta\right)$. The authors concluded their analysis with this interesting statement:

The branching fraction for production of atomic products has not been characterized, but indirect evidence indicates that production of diatomic fragments dominate for 193 and $248 \mathrm{~nm}$ photolysis.

Shortly after this paper, in the same year, another paper was published using the velocity map imaging technique, this time imaging recoiling $\mathrm{Cl}$ fragments [28]. The $\mathrm{Cl}\left({ }^{2} \mathrm{P}_{j}\right)$ atoms were also detected using a $2+1$ REMPI method, with the photolysis laser at near $235 \mathrm{~nm}$. The authors detected two states of $\mathrm{Cl}: \mathrm{Cl}^{*}\left({ }^{2} \mathrm{P}_{1 / 2}\right)$ and $\mathrm{Cl}\left({ }^{2} \mathrm{P}_{3 / 2}\right)$. The authors determined the branching ratio of $\mathrm{Cl}^{*} / \mathrm{Cl}$ to be $0.8 / 0.2$. Both $\mathrm{Cl}$ and $\mathrm{Cl}^{*}$ displayed bimodal energy distributions with the authors referring to the two peaks as "fast" and "slow" components. These bimodal energy distributions appear as rings of different diameter in 
velocity map images. The $\mathrm{Cl}^{*}$ had around $9 \%$ of the distribution in the "slow" channel, while $\mathrm{Cl}$ had nearly equal amounts of the fast and slow components (0.56 to 0.44 respectively). The properties of the "fast" channel agreed nicely with the known energetics of the well-studied ground state linear $\mathrm{N}_{3}\left(X^{2} \Pi\right)$ molecule. The "slow" component in the $\mathrm{Cl}$ distribution should correlate with a more energetic form of the $\mathrm{N}_{3}$ partner fragment, but the lowest level excited state of linear $\mathrm{N}_{3}$ was energetically out of reach by more than $2 \mathrm{eV}$. Another explanation had to be sought, and was found in a theory paper published by around the same time by M. Bittererova et al. [6]. The ultimate conclusion was that the "slow" component in the distribution was due to a previously unobserved excited state $\mathrm{N}_{3}\left({ }^{2} \mathrm{~B}_{1}\right)$, possessing a ring structure. Over the next five years, a great deal of evidence, both theoretical and experimental, has been found supporting the conclusion that this high energy form of $\mathrm{N}_{3}$ is, in fact, cyclic $\mathrm{N}_{3}$.

\subsection{Theoretical Studies of Cyclic Isomer}

The theoretical work published by M. Bittererova et al. in 2002 provides some interesting details about the possible properties of a cyclic $\mathrm{N}_{3}$ isomer [6]. The authors performed their calculations seeking to find a method to produce tetraazatetrahedrane $\left(\mathrm{N}_{4}\right.$ with a tetrahedral structure) for use as a high energy density material. Energetically, simply combining two $\mathrm{N}_{2}$ molecules is highly unfavored, so their focus turned to $\mathrm{N}_{3}+\mathrm{N}$ pathways. First they examined the stability of known $\mathrm{N}_{3}$ states and those not experimentally reported, but which may also be stable. They conclude that $\mathrm{N}_{3}$ in a ${ }^{2} \mathrm{~B}_{1}$ state possessing a ring geometry should be the lowest stable excited state of the $\mathrm{N}_{3}$ radical, located $29.9 \mathrm{kcal} / \mathrm{mol}$ above ground state linear $\mathrm{N}_{3}$. The ring structure calculated resembles an isosceles triangle with one short 1.218 angstrom bond and two 1.45 angstrom bonds. Their next calculations focused on whether any of the states determined to be stable could work as a viable precursor to $\mathrm{N}_{4}$. The calculations showed $\mathrm{N}\left({ }^{2} \mathrm{D}\right)+\mathrm{N}_{3}\left(\mathrm{C}_{2 v},{ }^{2} \mathrm{~B}_{1}\right) \rightarrow \mathrm{N}_{4}\left(\mathrm{~T}_{d},{ }^{1} \mathrm{~A}_{1}\right)$ to be a viable reaction pathway to produce $\mathrm{N}_{4}$. They also suggest that $\mathrm{N}_{2}+\mathrm{N}\left({ }^{2} \mathrm{D}\right)$ could be a reaction producing cyclic $\mathrm{N}_{3}$, proposing that a mixture of $\mathrm{N}_{2}$ and $\mathrm{N}\left({ }^{2} \mathrm{D}\right)$ will first react to produce $\mathrm{N}_{3}$ which then reacts with excess $\mathrm{N}\left({ }^{2} \mathrm{D}\right)$ to form $\mathrm{N}_{4}$.

Following the possible experimental observation of the cyclic state of the $\mathrm{N}_{3}$ radical by Hansen and Wodtke, K. Morokuma et al. did extensive further theoretical calculations focused on improving the quality of theoretical information about this system [68]. According to Morokuma:

Despite the large amount of theoretical work, there are still many unclear or misunderstood aspects of the electronic structure of the $\mathrm{N}_{3}$ radical, resulting from the spin- or symmetry-restricted nature of the previous calculations.

They calculated the potential energy surfaces of the ground doublet and quartet states and provide several important conclusions. First, they find their numerical results to be closer to the experimentally observed values, suggesting their calculation results are better than previously reported results. They report the cyclic $\mathrm{N}_{3}$ state to lie $30.3 \mathrm{kcal} / \mathrm{mol}$ 
above the ground linear state, with a barrier to isomerization between the two states at $32.1 \mathrm{kcal} / \mathrm{mol}$ above the cyclic state. Their work also casts doubt on Bittererova's assertion that $\mathrm{N}_{2}+\mathrm{N}\left({ }^{2} \mathrm{D}\right)$ is a viable pathway to forming $\mathrm{N}_{3}$. Finally, and in their own words "perhaps the most important outcome of this work", they find the barriers to dissociation and isomerization to lead to the conclusion that the cyclic $\mathrm{N}_{3}$ state "is quite stable and longlived under collision free conditions, even if formed with substantial internal energy." This conclusion supports the earlier experimental observations made by Hansen and Wodtke.

\subsection{More Experimental Observations}

\subsubsection{Ring Closing Dynamics of Hydrazoic Acid}

One year later, a collaborative paper between a number of researchers including the previously mentioned A.M. Wodtke and K. Morokuma was published combining high level quantum mechanical theory with a detailed experiment on the ring closing dynamics of $\mathrm{HN}_{3}$ [67]. In this work, the authors used hydrogen Rydberg atom time of flight (HRTOF) to analyze the energy of $\mathrm{H}$ atom product fragments in $\mathrm{HN}_{3}$ photodissociation across the 225-199 nm photolysis wavelength range. In this range, the authors note "a marked transition in the photochemical dynamics". The key observation of their experiment is a growing emphasis on a "slow" $\mathrm{H}$ atom channel as the available energy increases above $5.6 \mathrm{eV}$. This is analogous to the "slow" chlorine channel observed in the previous VMI $\mathrm{ClN}_{3}$ experiments, where the slow channel atoms are produced in conjunction with a high energy form of $\mathrm{N}_{3}$. The authors combined this result with high level quantum mechanical calculations of the $\mathrm{HN}_{3}$ potential energy surface. Interestingly, the onset of the appearance of the low translational energy $\mathrm{H}$ atom channel (and therefore the high energy $\mathrm{N}_{3}$ channel) corresponds with an $\mathrm{N}_{3}$ ring closing channel on the $\mathrm{S}_{1}$ potential energy surface. From this state, there is only a small barrier to direct dissociation to cyclic $\mathrm{N}_{3}+\mathrm{H}$.

The combination of high level theory with experimental results provided some of the best evidence yet as to the existence of a cyclic $\mathrm{N}_{3}$ species which had not been previously observed, even in a molecule as highly studied in photolysis experiments as $\mathrm{HN}_{3}[15,66]$.

\subsubsection{Photofragment Translational Spectroscopy}

Expanding upon their previous experimental work, Wodtke and co-workers performed several experiments to examine $\mathrm{ClN}_{3}$ photolysis in the context of photofragment translational spectroscopy (PTS). In many ways, the PTS technique provides a great deal of complimentary information to the VMI technique, making it a logical next step in further exploring this photolysis channel. The principles and experimental details of PTS will be covered in detail in Chapter 3.1 as they also form the basis of this dissertation work. An introduction is presented here to interpret the results of the $\mathrm{ClN}_{3}$ experiment described here and how these results lead into the exploration of $\mathrm{CH}_{3} \mathrm{~N}_{3}$ photochemistry.

Photofragment translational spectroscopy, like VMI, starts with a collision free molecular 
beam of the molecule of interest. The beam is again photolyzed and fragments ionized. The ionization, however, is not normally done through a REMPI scheme, as those are specific and selective to individual fragments. Instead, all products are ionized using techniques like electron bombardment ionization or intense light sources such as that found in synchrotron radiation. The ions are then filtered by mass to charge ratio $(\mathrm{m} / z)$ before being detected. Time of flight (TOF) spectra can be recorded for each $m / z$ for which ions are detected. These TOF spectra can be analyzed to determine the translational energy distribution of recoiling product fragments through methods which will be described in detail in Chapter 3 and 4 .

The advantage to this method is that all product fragments in the photolysis process can be observed, not just the $\mathrm{Cl}$ atom. In addition, momentum conservation laws allow unambiguous determination as to whether two observed fragments were created through the same photolysis pathway. This allowed for the recording of both the $\mathrm{Cl}$ and $\mathrm{N}_{3}$ fragment's TOF spectra, and thus observation and analysis of both product fragments in the same experiment.

The first experimental results from PTS on $\mathrm{ClN}_{3}$ were published in a letter in 2004 and focused on the branching ratio between the radical channel and the molecular channel [63]. The photolysis was carried out at $248 \mathrm{~nm}$ and fragments were observed at mass to charge ratios matching $\mathrm{N}^{+}, \mathrm{N}_{2}^{+}, \mathrm{Cl}^{+}, \mathrm{N}_{3}^{+}$and $\mathrm{NCl}^{+}$product fragments. Since this experimental technique is able to observe all fragments and ionization efficiency for each fragment in the electron impact ionization technique used can be calculated with good accuracy, it is possible to compare the total yield for each channel. Surprisingly, against all previous expectations, they found $95 \% \pm 3 \%$ of the photofragments came from the radical $\mathrm{N}_{3}+$ $\mathrm{Cl}$ channel (the remaining $5 \% \pm 3 \%$ belonged to the molecular elimination channel). It was noted in this paper that the $\mathrm{N}_{3}$ fragment was observed to have a bimodal energy distribution, but no significant analysis of this observation was included at the time.

Two more publications on PTS of $\mathrm{ClN}_{3}$ photolysis shortly followed in early 2005 [31, 56]. The first paper focused on determining the full primary and secondary dissociation pathways in $248 \mathrm{~nm} \mathrm{ClN}_{3}$ photolysis. Both the radical and molecular pathways were observed. Consistent with their previous observations, the $m / z=35$ fragment $\left(\mathrm{Cl}^{+}\right)$was observed to have a bimodal distribution. In addition, the momentum matched partner fragment at $m / z=42\left(\mathrm{~N}_{3}^{+}\right)$was also observed with a bimodal distribution. While the cyclic $\mathrm{N}_{3}$ state was the best explanation of the high energy $\mathrm{N}_{3}$ fragment, it had not been identified conclusively, so the authors refer to the higher energy fragment as the High Energy Form of $\mathrm{N}_{3}$ or $\mathrm{HEF}-\mathrm{N}_{3}$. They conclude that the $\mathrm{HEF}-\mathrm{N}_{3}$ shares many expected characteristics with the theory predictions for cyclic $\mathrm{N}_{3}$. For instance, the energy difference determined between linear and $\mathrm{HEF}-\mathrm{N}_{3}$ was $30.1 \pm 3 \mathrm{kcal} / \mathrm{mol}$, which is in excellent agreement with Bittererova's previous calculation of $29.9 \mathrm{kcal} / \mathrm{mol}$ and Morokuma's calculation of $30.3 \mathrm{kcal} / \mathrm{mol}$. It should be pointed out that the $\mathrm{HEF}-\mathrm{N}_{3}$ was stable enough to be detected by electron impact ionization on a time scale of more than 100 psec after its formation. Secondary decomposition channels were also observed coming from both $\mathrm{NCl}$ and $\mathrm{N}_{3}$. The amount of detected $\mathrm{N}_{3}$ strongly deviated from the expectation provided by the momentum matched $\mathrm{Cl}$ partner fragment when the $\mathrm{N}_{3}$ fragment's internal energy was 
$11 \pm 2 \mathrm{kcal} / \mathrm{mol}$ above the cyclic $\mathrm{N}_{3}$ minimum. The explanation for this observation is that $\mathrm{N}_{3}$ fragments above this energy undergo unimolecular decomposition into $\mathrm{N}+\mathrm{N}_{2}$. The observed $m / z=14$ and 28 fragment's TOF spectra were able to be fit to a variety of secondary decomposition mechanisms including this unimolecular dissociation of $\mathrm{HEF}-\mathrm{N}_{3}$.

The second full paper stemming from the PTS of $248 \mathrm{~nm}$ photolysis of $\mathrm{ClN}_{3}$ focused specifically on the radical channel and, in addition to the derived bimodal translational energy release, presented data on near threshold photoionization of the $\mathrm{N}_{3}$ fragment. The instrument used in this experiment differed slightly from the one used in the previous paper. Most significantly, this experiment used tunable synchrotron radiation to ionize fragments as opposed to the electron impact ionization used previously. Aside from the already excellent agreement of the energetics to the predicted thermodynamic characteristics of cyclic $\mathrm{N}_{3}$, the authors determined the ionization threshold of the $\mathrm{HEF}-\mathrm{N}_{3}$ to be $10.6 \mathrm{eV}$, which is also in good agreement with theoretical predictions for the ionization potential of cyclic $\mathrm{N}_{3}$ from the ground state neutral to the lowest cationic state [3]. The observed threshold for ground state linear $\mathrm{N}_{3}$ was $11.06 \mathrm{eV}$ which is also in good agreement with the known ionization potential [22].

\subsubsection{More Velocity Map Imaging Experiments}

The results of another VMI experiment were published in 2006 by Samartzis and Wodtke, once again with $\mathrm{ClN}_{3}$ photolysis [52]. Here several different photoionization wavelengths were used in the 234 to $280 \mathrm{~nm}$ range - 234, 240, 266, and $280 \mathrm{~nm}$. The experimental details are similar to the previously reported VMI experiments. The key observation made in this paper was an apparent appearance potential for $\mathrm{HEF}-\mathrm{N}_{3}$ in $\mathrm{ClN}_{3}$ photolysis between $248 \mathrm{~nm}$ and $260 \mathrm{~nm}$. Their results showed the same clear bimodal distribution of $\mathrm{Cl}$ as previous work in the 234 and $240 \mathrm{~nm}$ velocity map images. However the bimodal distribution was not present in the 266 and $280 \mathrm{~nm}$ images, only a single peak whose energy matched well with the linear state of $\mathrm{N}_{3}$ was observed. These results alone imply a threshold for the appearance of HEF- $\mathrm{N}_{3}$ between 240 and $260 \mathrm{~nm}$. However, they previously observed the $\mathrm{HEF}-\mathrm{M}_{3}$ at $248 \mathrm{~nm}$ in the PTS experiments, thereby narrowing the appearance range further. They use this information to set the appearance threshold at $4.83 \pm 0.17 \mathrm{eV}$ photolysis energy.

\subsubsection{Exclusive Production of Cyclic Isomer}

The final publication produced prior to the performance of this dissertation work, observing $\mathrm{HEF}-\mathrm{N}_{3}$ in the photolysis of $\mathrm{ClN}_{3}$, was published by Samartzis and Wodtke in 2007 [55]. This time they studied the $157 \mathrm{~nm}$ photolysis of $\mathrm{ClN}_{3}$ by PTS under collision free conditions. The analysis showed it was likely that photolysis of $\mathrm{ClN}_{3}$ at this wavelength exclusively produces the HEF-N ${ }_{3}$. Significantly, the authors more firmly begin to refer to the $\mathrm{HEF}$ of $\mathrm{N}_{3}$ as cyclic $\mathrm{N}_{3}$ in this paper, as they now have published the results of four experiments, performed at different photolysis wavelengths, which all display a high energy 


\subsection{More Experimental Observations}

$\mathrm{N}_{3}$ species with a defined heat of formation which matches well with several theoretical predictions for the cyclic $\mathrm{N}_{3}$ isomer. 
Chapter 2 Previous Work and Literature Review 


\section{Chapter 3}

\section{Fundamentals}

The chapter formulates the fundamental description of the experimental techniques used in this part of my dissertation. It begins by covering the principles and physics related to the techniques of photofragment translational spectroscopy and continues with an overview of the generation and use of synchrotron radiation light sources.

\subsection{Photofragment Translational Spectroscopy}

Photofragment translational spectroscopy is a useful technique for studying the dynamics of a photochemical process in a collision free environment. The term refers to the process of experimentally measuring the translational energy probability distribution of product fragments in a photodissociation process on a cold molecular beam. The technique and instrumentation was first described by Kent Wilson and co-workers in 1970 [10] and has been used to study the photochemical dynamics of a wide variety of molecules since then.

\subsubsection{Important Concepts}

\section{Laboratory to Center of Mass Frame Conversion}

Photofragment translational spectroscopy relies on measuring the flight times of known mass photodissociation product fragments in the laboratory frame of reference and extracting information about the center of mass energy distribution. Figure 3.1 illustrates a general Newton diagram of the relevant velocity vectors in a PTS experiment for the dissociation process $\mathrm{AB} \stackrel{h \nu}{\longrightarrow} \mathrm{A}+\mathrm{B}$. $v_{A B}$ is the laboratory velocity of the parent fragment in the molecular beam. $v_{A}$ is the velocity of the product fragment $\mathrm{A}$ in the laboratory frame, which is derived from the time of flight measurement. $u_{A}$ is the center of mass velocity of the product fragment. The angle $\Theta$ is the angle between the molecular beam and the detector and is referred to as the lab angle. From these quantities and the masses of product fragments $\mathrm{A}$ and $\mathrm{B}$, a wide range of information about the dynamics of the dissociation process can be extracted.

The conversion between the measured lab velocity to the center of mass velocity of the products can be done from simple vector math. From the illustration in Figure 3.1, it is clear that the $x$-component of the product fragment's velocity is the same in both the 


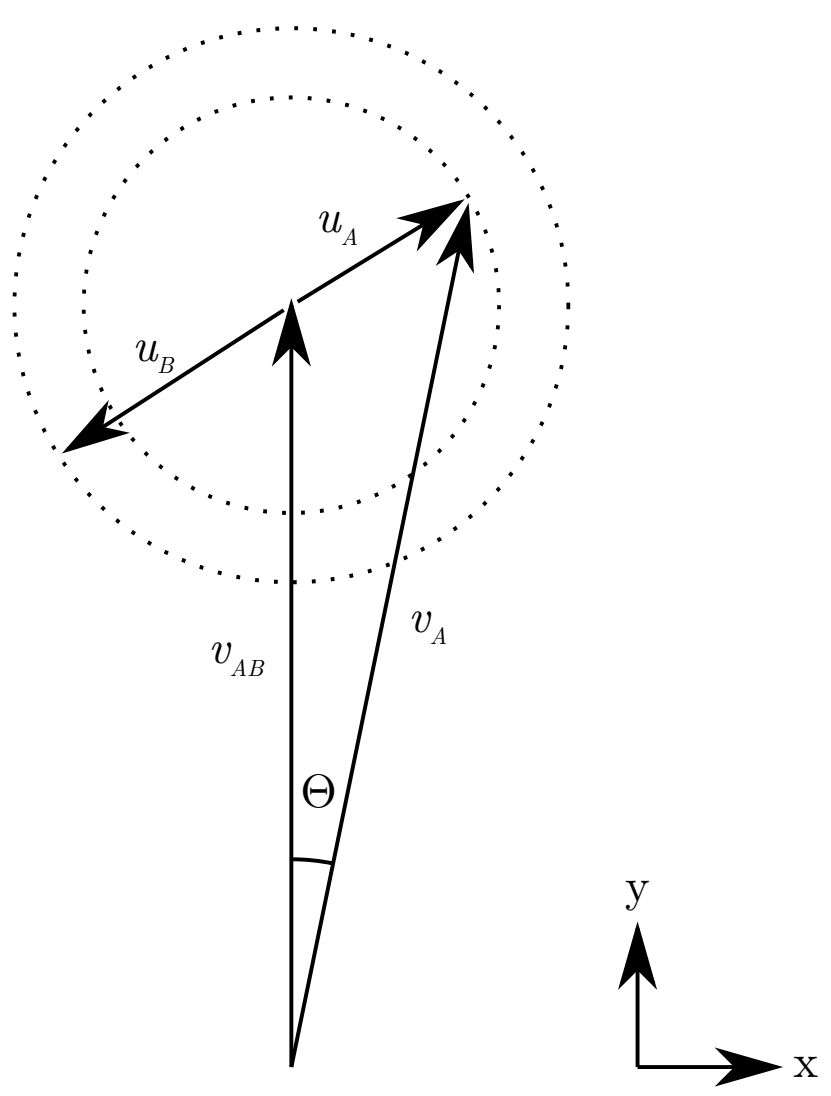

Figure 3.1: Newton Diagram showing the relationship between the lab and center of mass velocity frames of reference for the photodissociation process $\mathrm{AB} \stackrel{h \nu}{\longrightarrow} \mathrm{A}+\mathrm{B}$ in photofragment translational spectroscopy.

center of mass and lab frames:

$$
v_{A x}=u_{A x}
$$

In this particular diagram, the $y$-component of the lab velocity has a slight forward acceleration compared to the parent molecule and can always be described as the sum of the $y$-component of the center of mass velocity and the lab velocity of the parent fragment:

$$
v_{A y}=u_{A y}+v_{A B}
$$

The $x$ - and $y$-components of the lab velocity of the product fragment can also be related to the measured velocity by trigonometry using the detector angle, $\Theta$ :

$$
\begin{aligned}
& v_{A x}=v_{A} \sin \Theta \\
& v_{A y}=v_{A} \cos \Theta
\end{aligned}
$$


Finally, the center of mass velocity of fragment A can be determined by:

$$
\begin{aligned}
u_{A} & =\sqrt{u_{A x}^{2}+u_{A y}^{2}} \\
& =\sqrt{\left(v_{A} \sin \Theta\right)^{2}+\left(v_{A} \cos \Theta-v_{A B}\right)^{2}}
\end{aligned}
$$

In summary, by measuring the time of flight of fragment $\mathrm{A}$ over a known distance to a detector at known lab angle $\Theta$, the lab velocity, $v_{A}$, is known and the center of mass velocity of the recoiling fragment, $u_{A}$, can be calculated. These velocity distributions can then be converted into translational energy distributions as described in the following section.

\section{Momentum Matching}

An essential concept in analyzing photofragment translational spectroscopy data is the "momentum matching" principle. In the dissociation process, the two product fragments $\mathrm{A}$ and $\mathrm{B}$ must abide by the law of conservation of momentum, and:

$$
\begin{aligned}
p_{A} & =-p_{B} \\
m_{A} u_{A} & =-m_{B} u_{B}
\end{aligned}
$$

As a consequence, once the velocity distribution of one fragment is known, the velocity of the other fragment is known as well. This leads directly to knowledge about the energetics of the dissociation process as follows.

$$
E_{T}=\frac{1}{2} m_{A} u_{A}^{2}+\frac{1}{2} m_{B} u_{B}^{2}
$$

From Equation 3.6:

$$
\begin{aligned}
& u_{A}=-\frac{m_{B} u_{B}}{m_{A}} \\
& u_{B}=-\frac{m_{A} u_{A}}{m_{B}}
\end{aligned}
$$

Substituting either of these into Equation 3.7 gives:

$$
\begin{aligned}
& E_{T}=\frac{1}{2} m_{A} u_{A}^{2}\left(\frac{m_{A}+m_{B}}{m_{B}}\right) \\
& E_{T}=\frac{1}{2} m_{B} u_{B}^{2}\left(\frac{m_{A}+m_{B}}{m_{A}}\right)
\end{aligned}
$$

As the product masses, $m_{A}$ and $m_{B}$, are known, once the center of mass velocity, $u$, of either fragment is determined, the translational energy can be derived from either Equation 3.11 or 3.12 . In a real dissociation process, the product fragments typically do not have one single velocity, but some statistical distribution of velocities. As a result, the real quantity derived from a PTS experiment is actually a probability distribution function of 


\section{Chapter 3 Fundamentals}

translational energies, $P\left(E_{T}\right)$.

The actual analysis is done in reverse, due to the challenges of direct mathematical inversion on noisy real world experimental data. Instead, a $P\left(E_{T}\right)$ is first "guessed" and the time of flight distribution which would result from that energy distribution with the instrumental parameters is calculated and compared with the experimental results. The energy distribution is iterated until it produces a TOF spectra which matches the experimental results. The procedure is known as "forward convolution fitting" and will be discussed in more detail in Chapter 4.3.2.

A further consequence of the momentum matching principle is that if a single translational energy distribution derived in this way can be used to fit the time of flight spectra of two product fragments, then those two product fragments must have originated in the same dissociation process. This is an essential point and will be relied upon heavily in the analysis of results in Chapter 5.

Much more information can be extracted from this principle, for example, from the angular distributions of product fragments. However this goes beyond the scope of information necessary for interpreting the results of this dissertation.

\subsubsection{Typical Experimental Procedure}

The technique of photofragment translational spectroscopy has been widely used for decades and is now a well-established technique. While there can be some variation in the instrumental setups, I will focus on the configuration which was used for my experiment. The typical methodology of a PTS experiment is as follows:

- A molecular beam of the molecule of interest is produced by skimming a pulsed valve source, providing a cold beam of molecules in a collision free environment. The molecule of interest is diluted in an inert carrier gas, such as helium or argon, which has the effect of cooling the internal degrees of freedom and producing a more consistent molecular beam velocity distribution. This is important as having a consistent velocity distribution is necessary for the forward convolution fitting.

- The molecular beam is photolyzed by a pulsed laser source. The resulting product fragments recoil away from each other with an unknown center of mass velocity. The velocities of these fragments are related to each other through conservation of momentum as describe in the previous section.

- The product fragments travel over a calibrated flight distance, allowing different velocity fragments to spread out in the spatial dimension. After this free flight region, fragments are ionized. In the research presented here, synchrotron light was used as the ionization source. There are several advantages to using a synchrotron light source as the ionization method. These will be discussed in detail in the next section. 
- Once ionized, the products can be filtered by mass to charge ratio using traditional mass spectroscopy methods. In this work, a differentially pumped "universal detector" based on quadrupole mass spectrometry is utilized. This type of detector was introduced by Y.T. Lee and was a significant contribution to the field [40]. By scanning the mass filter, it is possible to determine the $m / z$ of all products which are present in the dissociation. Once the product $m / z$ values are known, the filter can be tuned to transmit a single $m / z$ to the detector. The time of flight spectra are recorded for each fragment, leading to information about the center of mass velocity distribution of each of the products.

- The angle of the detector relative to the molecular beam can be changed, allowing the angular distribution of products to be measured as well. Depending on the velocities of the recoiling fragments, some angles may make it easier to observe a particular energy state of the product fragments. From Figure 3.1, it is clear that the smaller a center of mass velocity for a product fragment is, the smaller the lab angle needed to detect that fragment is.

\subsection{Synchrotron Radiation}

In my experiment, synchrotron radiation was used to ionize the product fragments of the photodissociation. Some understanding of the origin of synchrotron radiation is necessary to appreciate how its properties are advantageous in this type of experiment. A brief historical and theoretical description will be presented, followed by a description of the essential components to generate and control synchrotron radiation and, finally, how its properties contributed to the experiment.

\subsubsection{Theoretical Description and Discovery}

Synchrotron radiation is composed of high energy photons which are radiated from electrons traveling near the speed of light. Though not named synchrotron radiation at the time, this phenomenon was theoretically predicted as early as 1898 by A. Lienard [42]. Several others contributed to the theory of how electrons moving in a circular path should radiate energy before the first experimental observations. One of the last theoretical descriptions before its direct observation was made by D. Ivanenko and I. Pomeranchuk in 1944 [36]. In this letter, the two authors made the following statement:

Electrons moving in a magnetic field will be accelerated and must radiate in accordance with classical electrodynamics.

The formula derived for this radiated energy is:

$$
-\left(\frac{\partial E}{\partial X}\right)=\frac{2}{3}\left(\frac{e^{2}}{m c^{2}}\right)^{2}\left(\frac{E}{m c^{2}}\right)^{2}\left(\frac{\mathbf{V} \times \mathbf{H}}{c}\right)^{2}
$$




\section{Chapter 3 Fundamentals}

where $E$ is the energy of the electron, $\mathbf{V}$ is the velocity, $m$ the mass and $\mathbf{H}$ the magnetic field.

When the electrons are traveling near the speed of light $(|\mathbf{V}| \rightarrow c)$ and the magnetic field is perpendicular to the direction of the velocity, the formula simplifies to

$$
-\left(\frac{\partial E}{\partial X}\right)=\frac{2}{3}\left(\frac{e^{2}}{m c^{2}}\right)^{2}\left(\frac{E H}{m c^{2}}\right)^{2}
$$

Note that the intensity of the radiation produced in this formula should depend on the electron energy, $E$, and the magnitude of the magnetic field, $H$.

This phenomenon was soon after surreptitiously observed and reported by F. Elder, A. Gurewitsch, R. Langmuir, and H. Pollock working in a General Electric lab in the late 1940s [23]. The effect was noticed when working with GE's $70 \mathrm{MeV}$ synchrotron which was built in 1946 (hence the origin of the name). The early studies of synchrotron radiation saw it as an unwanted side effect of particle acceleration, as the loss of energy through synchrotron radiation makes it more and more difficult to accelerate particles to increasingly higher velocities. Years later, the beneficial properties of synchrotron radiation were proposed and facilities began opening to exploit this phenomenon for new kinds of research.

\subsubsection{Essential Components of a Synchrotron}

Synchrotron radiation is generated by an electron beam being bent in a magnetic field while traveling near the speed of light. While there are some variations in equipment amongst individual facilities, what follows is a general overview of components essential to the production and use of synchrotron radiation in modern $3^{\text {rd }}$ generation light sources (which includes the National Synchrotron Radiation Research Center in Hsinchu, Taiwan, where this experiment was conducted).

- An electron beam is created and injected into in a linear accelerator where it accelerates to a high energy, and then continues into a booster ring where it can reach an even higher energy. Most modern facilities produce an electron beam in this stage with energies between 1 and $3 \mathrm{GeV}$.

- The high energy electron beam is transported into a storage ring. The storage ring consists of a number of components which improve the characteristics of the radiation and prolong its lifetime. This so-called "ring" is actually a many-sided polygon consisting of linear regions connected via a bending magnet located at each vertex. The electrons are bent at these vertices producing the synchrotron radiation which continues tangentially from the curved path of the electron beam. The linear portions of the storage ring include quadrupole and sextupole magnets for collimating the electron beam, radio-frequency ( $\mathrm{RF}$ ) cavities for re-accelerating the beam as it will continually lose energy to emission of synchrotron radiation, and insertion devices designed to boost the spectral brightness and characteristic energy of the synchrotron radiation. 
- The RF cavities give a pulsed time structure to the synchrotron radiation. These cavities form potential wells to accelerate the electrons inside. The width of these potential wells leads to "bunches" of electrons being accelerated simultaneously. This bunching leads to the time structuring of the emitted radiation. Typically, the pulses are very short - giving another unique characteristic which can be advantageous in certain experiments.

- Typically, the energy spectrum of the synchrotron radiation is fixed for a given facility. Recall from Equation 3.14 that the energy of the emitted radiation depends on the energy of the electrons (a property which is limited by the facility's accelerator and booster) and the strength of the bending magnetic field (limited by the geometry of the storage ring). $3^{\text {rd }}$ generation synchrotron light sources circumvent this limitation by the use of insertion devices. An undulator is one type of insertion device consisting of a series of alternating polarity magnets spaced by some user adjustable narrow gap. The electron beam obtains some periodic oscillation in this field, resulting in an interference pattern in the radiation. This effect produces wavelengths of radiation with greatly increased spectral brightness. By adjusting the gap in the undulator, the magnetic field strength changes, changing the energy of the resulting synchrotron radiation. This device makes it possible to tune the energy of the ionizing radiation over a wide range during the experiment.

\subsubsection{Advantages of Synchrotron Light Sources}

Synchrotron radiation has a number of unique and interesting properties. Among them are a wide range, continuous spectrum which can span from the far infrared to near gamma ray energies with high brightness, stability and a pulsed time structure. For this experiment, synchrotron radiation is used as the ionization source for the product fragments formed in the photodissociation process. There are several advantages to using synchrotron light as an ionization source:

- This method provides an intense source of single photons with high enough energy to ionize product fragments. Compared to resonantly enhanced multi-photon ionization, this has the advantage of being applicable to any product fragments formed.

- This is a "softer" ionization method than electron impact ionization. Electron bombardment can often produce secondary dissociation events which are unrelated to the photodissociation dynamics. By using the synchrotron, it is more likely that observed dynamics are the result of the photodissociation and not the ionization process.

- The photoionization energy can be tuned simply by adjusting the gap in the undulator at the beamline. This can have several applications in the experiment. First, it can allow fragments with the same mass, but different ionization potentials to be distinguished by adjusting the energy of the ionizing radiation. Second, it can allow for photoionization threshold measurements to be performed. By increasing 
Chapter 3 Fundamentals

the photoionization energy in small steps and measuring time of flight spectra, the appearance threshold for a particular fragment can be determined. This has applications in the identification of the structure of the $\mathrm{N}_{3}$ fragment presented in Chapter 5 . 


\section{Chapter 4}

\section{Methods}

This chapter explains specific experimental details and the analytical methods related to the experiment. I start with the synthesis and characterization methods of the molecule of interest, methyl azide, $\mathrm{CH}_{3} \mathrm{~N}_{3}$. Then I will cover the details of the experimental apparatus used to conduct the PTS experiment and the synchrotron facility where the research was performed. Finally, I will cover the forward convolution fitting method used to analyze the results obtained in the experiment.

\subsection{Synthesis and Characterization of Samples}

The methyl azide samples used in this experiment were synthesized using a simple substitution reaction between sodium azide, $\mathrm{NaN}_{3}$, and methyl iodide, $\mathrm{CH}_{3} \mathrm{I}$.

$$
\mathrm{NaN}_{3}+\mathrm{CH}_{3} \mathrm{I} \stackrel{90^{\circ} \mathrm{C}}{\longrightarrow} \mathrm{CH}_{3} \mathrm{~N}_{3}+\mathrm{NaI}
$$

Small quantities (1 to $2 \mathrm{~mL}$ ) were produced at a time to minimize the explosive risk of azide compounds and to use freshly synthesized samples as much as possible. I have previously seen methanol contamination in larger methyl azide samples (identified by NMR). Methanol can be formed through the reaction of condensed water vapor into the $<0^{\circ} \mathrm{C}$ sample flask whenever it is exposed to atmosphere. For these reasons, several smaller samples were produced as needed, rather than one large batch to cover the entire experiment.

The sodium azide (4.9 grams) and methyl iodide $(4.7 \mathrm{~mL})$ were mixed in a $4: 1$ ratio mixture of dimethyl formamide (DMF) and water $(60 \mathrm{~mL}$ total). This method is adapted from the method of Bock and Dammel for synthesizing alkyl azides via simple substitution reactions [7]. Methyl iodide was chosen as the alkyl source over the more common dimethyl sulfate due to safety considerations. A number of explosions have been reported during methyl azide synthesis [59]. The mixture was heated to $90^{\circ} \mathrm{C}$ for several hours in a low-flow nitrogen environment while being stirred with a magnetic stir bar. The flow of nitrogen gas through the reactant flask was essential to obtain any product yield. The methyl azide product has a boiling point around $20^{\circ} \mathrm{C}$ [9] and therefore is expected to be gaseous under these conditions. Methyl iodide has a boiling point of around $42^{\circ} \mathrm{C}$ [8], so it is also possible under these conditions to have some unreacted $\mathrm{CH}_{3} \mathrm{I}$ vapor make it into the product flask. The gaseous product was cooled in a water-jacketed condenser, and collected in a flask 
submerged in a dry ice/acetone bath at a temperature of $-70^{\circ} \mathrm{C}$. The final product was immediately transferred into the glass bubbler used in the experiment and stored in a refrigerator at $0^{\circ} \mathrm{C}$ until used, typically less than 24 hours after synthesis. This procedure was repeated several times as needed to produce enough product to carry out the complete experiment.

A portion of a representative sample of methyl azide produced with this method was placed in an NMR tube for composition analysis. Methyl azide has an expected ${ }^{1} \mathrm{H}$ NMR peak at $2.98 \mathrm{ppm}$ [33]. Methyl iodide has an expected peak at $2.19 \mathrm{ppm}$. Both of these peaks were observed in the sample's NMR spectra. The calculated ratio between the integrated area of the $\mathrm{H}$ peaks suggests greater than $90 \%$ of the sample was methyl azide with the remainder being methyl iodide. There was no observation of the DMF solvent in the product NMR spectra and no methanol was detected in the freshly synthesized samples which were not exposed to atmospheric gases.

\subsection{Experimental Instrumentation}

The National Synchrotron Radiation Research Center (NSRRC), located in Hsinchu, Taiwan, is a $3^{\text {rd }}$ generation synchrotron light source which began operation in 1993.

The injector is the source of the high energy electrons which produce the synchrotron light. The NSRRC's injector consists of a linear accelerator which accelerates the electrons to $50 \mathrm{MeV}$ leading into a booster ring with a circumference of $72 \mathrm{~m}$ which further accelerates the electrons to $1.5 \mathrm{GeV}$ - the maximum operating energy for this facility. These high energy electrons are injected into the storage ring via a transport line, where they produce synchrotron light for use by users at the more than 50 experimental end-stations on 28 beamlines the facility currently has available.

Beamline 21A, where these experiments were performed, is known as the Chemical Dynamics beamline. It has synchrotron light available in an energy range from 4 to $500 \mathrm{eV}$ with a resolution $(E / \Delta E)$ of 50 . The energy is user adjusted by changing the gap of an undulator as explained in Chapter 3.

The instrument at the beamline itself has been described in detail elsewhere [44, 64] and the experimental method mostly follows the methods for PTS described in Chapter 3, but an overview of the more important features and components relevant to understanding the results will be covered again. A schematic diagram of the instrument can be found in Figure 4.1. The instrument is a crossed molecular beam source with two pulsed molecular beam sources arranged at a $90^{\circ}$ angle to each other. Only one source was necessary to perform this experiment. The beam source can be rotated with respect to a fixed detector allowing measurements of product fragments at different lab angles. The majority of the data in this series of experiments was obtained at a $30^{\circ}$ angle between source and detector with some data acquired at $60^{\circ}$. The methyl azide sample bubbler was kept refrigerated at a temperature of $-15^{\circ} \mathrm{C}$, which produced a corresponding vapor pressure for methyl azide measured to be around 150 torr. Helium was used as a carrier gas and was passed through the sample bubbler with the total backing pressure of gas entering the source chamber 


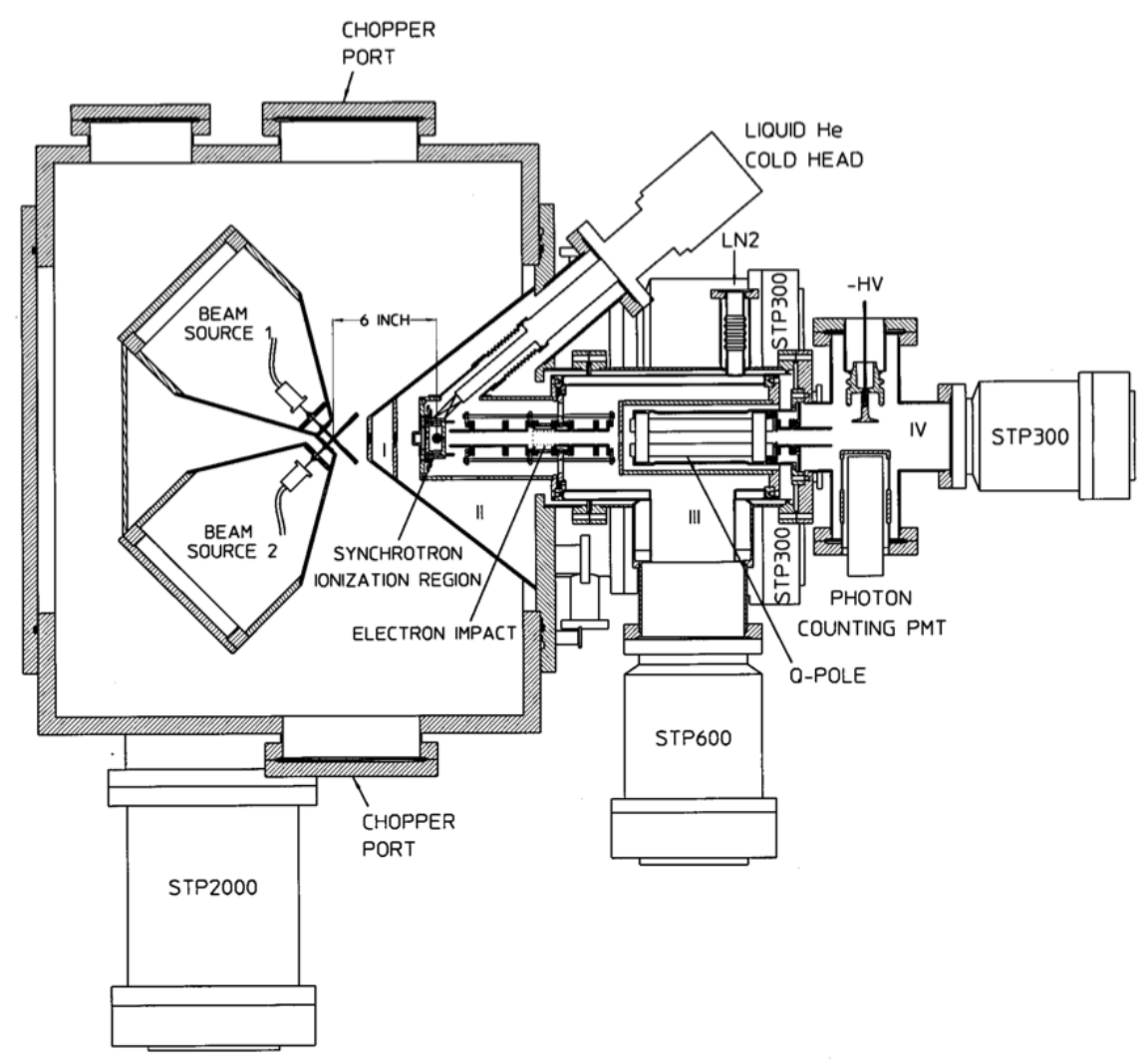

Figure 4.1: Schematic drawing representing the instrument used at the Chemical Dynamics Beamline 21A at the NSRRC in Hsinchu, Taiwan. Reproduced from Reference [64].

averaging around 260 torr throughout the experiment.

The molecular beam was generated using a solenoid pulse valve (a low repetition rate Even-Lavie (EL) valve [24] for the $248 \mathrm{~nm}$ photolysis experiment, and a commercial General valve for the $193 \mathrm{~nm}$ photolysis experiment). The EL valve was set to a voltage of $1.25 \mathrm{~V}$, an open time of $25 \mu \mathrm{sec}$ and a repetition rate of $50 \mathrm{~Hz}$. The General valve had a voltage of $200 \mathrm{~V}$, open time of $90 \mu \mathrm{sec}$, and a $50 \mathrm{~Hz}$ repetition rate. There was no difference observed between the two valves. Initially the EL valve was used to minimize the production of methyl azide clusters. However, significant clustering was not a problem and the subsequent $193 \mathrm{~nm}$ photolysis experiment was done with the General valve.

The methyl azide beam was intersected at a $90^{\circ}$ angle by an unpolarized, focused, pulsed excimer laser. $\mathrm{KrF}$ was used to generate a $248 \mathrm{~nm}$ photolysis wavelength and ArF to generate a $193 \mathrm{~nm}$ photolysis wavelength. The laser spot size was $8.5 \mathrm{~mm}$ by $2.5 \mathrm{~mm}$ for the $248 \mathrm{~nm}$ photolysis and $8 \mathrm{~mm}$ by $3.5 \mathrm{~mm}$ for the $193 \mathrm{~nm}$ photolysis with the beam spot being narrower in the TOF direction in both cases.

The product fragments resulting from the photolysis travel a $10.05 \mathrm{~cm}$ flight distance 
where they are ionized by a focused, tunable beam of synchrotron radiation. The fragment ions are then filtered according to their mass to charge ratio (referred to as $\mathrm{m} / \mathrm{z}$ ) in a quadrupole mass filter (QMF) before reaching a Daly style detector for ion counting [18]. When an ion strikes the detector, high energy electrons are emitted from the surface toward a phosphor screen. The phosphor screen is covered by a thin sheet of metal to reduce background noise. The high energy electrons are easily able to penetrate the thin metal sheet and strike the phosphor screen, producing photons. These photons are amplified into a measurable electric signal through a photomultiplier tube. The data was averaged, typically over 20,000 to 80,000 laser shots, using a multi-channel scaler (MCS).

The ionizing synchrotron radiation could be tuned by varying the gap in the beamline's undulator. This allows a wide range of photoionization energies to be used, which is useful for distinguishing fragments with the same masses but different ionization potentials. The experimental results reported here were carried out for photoionization energies of 11.9, $12.83,13.3,15.9$ and $17.05 \mathrm{eV}$. The energy resolution of this radiation was $3 \%$. High energy photons which are produced from higher order harmonics of the beamline undulator are suppressed by use of a gas cell filled with inert gas which can pass the desired ionization energy, but absorbs higher energy photons. The gases used in this cell were krypton, argon or neon, depending upon the energy of the ionizing radiation being used.

\subsection{Data Analysis}

\subsubsection{Pre-Processing}

All the acquired data was pre-processed in the same way. First, the average background signal was subtracted from all points. In the case of some $m / z$ which are expected to have a high presence in the residual gas of the instrument, such as 28 from CO outgassing, a background spectrum with no molecular beam photolysis was recorded for subtraction purposes. The intensity (number of counts recorded in each channel) of the data was normalized based on the laser power, synchrotron radiation intensity and the total number of laser shots used to acquire the spectra. Finally, a time offset in the triggering of the laser pulses relative to the pulse valve opening was taken into account and the times of flight modified to correct for this.

\subsubsection{Principles of Forward Convolution Fitting}

The time of flight data was analyzed by the forward convolution fitting method standardly employed for data analysis in this type of experiment. This method takes known information about the instrumental parameters in the experiment and simulates time of flight results with a high degree of accuracy. The only unknown parameter needed to simulate an accurate time of flight is the translational energy release probability, $P\left(E_{T}\right)$, for a given dissociation pathway. A $P\left(E_{T}\right)$ can be guessed and a TOF spectrum simulated. The $P\left(E_{T}\right)$ is incrementally adjusted until the simulated spectrum matches the observed experimental 
time of flight spectrum. The known input parameters used to simulate the experiment include the molecular beam's velocity and angular distribution, the flight distance to the detector, the laboratory angle between molecular beam and detector, the size and intensity of dissociation laser and ionizing synchrotron radiation, and the detector's angular acceptance width. The simulated time of flight function is convoluted with functions derived from these parameters, in effect accurately broadening the simulated peaks in the TOF spectrum in a way consistent with the experimental conditions.

A pair of ions with different masses originating from the same dissociation pathway are expected to have the same momentum due to conservation laws (see Chapter 3). For this momentum matched pair of products, one $P\left(E_{T}\right)$ will correctly simulate the time of flight spectrum of both. In this situation, it can then be concluded that the two product fragments originated from the same dissociation pathway and information can be obtained about which dissociation pathways occur in the photolysis.

For this experiment, an anisotropy parameter $\beta=0$ was used in the analysis, corresponding to an isotropic distribution, due to the use of unpolarized lasers in the photolysis.

The direct result of the forward convolution fitting is the center of mass translational energy probability distribution, $P\left(E_{T}\right)$, of the dissociation channel. This result by itself does not have much meaning - it must be analyzed in the context of the possible dynamics of the reaction channel. Interpretation of the derived $P\left(E_{T}\right)$ for each set of time of flight data makes up the bulk of the discussion and analysis of results in Chapter 5 .

\subsubsection{Computer Software for Forward Convolution Fitting}

The primary software used to perform the forward convolution fitting is PHOTRAN, written by Stephen Harich [32]. This Windows based program performs forward convolution fitting using the previously described parameters as inputs with a graphical user interface (GUI) for iterating the $P\left(E_{T}\right)$ points.

PHOTRAN is only capable of modeling a primary dissociation event. It is possible that following the initial photolysis, one or both of the resulting fragments can further dissociate into additional products. For the secondary product fragments which originate in these events, the time of flight data is analyzed by ANALMAX, a Fortran compiled program written by Alec M. Wodtke [61]. ANALMAX uses an algorithm similar to PHOTRAN with similar input parameters describing the experiment to simulate the primary dissociation event. However, it can go a step further and simulate a secondary dissociation process using an additional $P\left(E_{T}\right)$ for the secondary process along with the $P\left(E_{T}\right)$ for the primary dissociation step, selecting the mass of the parent ion, and the expected masses of the secondary dissociation products. This fitting is done using a more complicated algorithm involving several additional calculation steps which also leads to a more time and memory consuming process for each small change to the secondary $P\left(E_{T}\right)$. It is less user friendly, lacking a GUI and using plain text files as the input and output. For these reasons, while ANALMAX is capable of determining the primary dissociation with similar accuracy, PHOTRAN was kept as the method of choice for modeling primary distributions and ANALMAX was only used in events which could not be applied to PHOTRAN. 
Chapter 4 Methods 


\section{Chapter 5}

\section{Results and Discussion}

In this section I will present the results of the experiment and my interpretation of these observations. The data and analysis for each photolysis wavelength will be combined and will be presented in two sections - the first covering $248 \mathrm{~nm}$ photolysis and the second covering $193 \mathrm{~nm}$ photolysis. These results have been published in two papers which can be found in References [38, 39].

\subsection{Possible Dissociation Pathways}

Several experiments have been performed previously examining methyl azide decomposition, though under different conditions from this work. These experiments can provide a basis for what mechanisms may be expected to be observed in my work. Methyl azide pyrolysis was first reported in 1933 by John Leermakers [41]. A more recent pyrolysis experiment was done by Bock and Dammel in 1987 [7]. In pyrolysis, the authors found the thermal decomposition of methyl azide to begin around $720 \mathrm{~K}$. At this point, they observe evidence of methanimine, $\mathrm{CH}_{2} \mathrm{NH}$. Further decomposition of methanimine into $\mathrm{H}_{2}$ and $\mathrm{HCN}$ also becomes possible at this point. Methyl azide photolysis has been experimentally performed as well, though in an environment which allows for molecular collisions [16]. The photolysis wavelengths that have been used are between $292 \mathrm{~nm}$ and $325 \mathrm{~nm}$. The authors found it likely that methanimine was produced by a concerted $\mathrm{N}_{2}$ elimination with a $1,2 \mathrm{H}$ atom shift, bypassing singlet methylnitrene, ${ }^{1} \mathrm{CH}_{3} \mathrm{~N}$, as an intermediate. They also find evidence for the spin-forbidden triplet methylnitrene radical, ${ }^{3} \mathrm{CH}_{3} \mathrm{~N}$. From the previous work on $\mathrm{ClN}_{3}$ presented in Chapter 2, I expect the $\mathrm{C}-\mathrm{N}$ bond rupture channel producing the $\mathrm{N}_{3}$ radical to be possible as well.

\subsubsection{Primary Dissociation Pathways}

From these previous observations, there are several possible reaction channels which can be reasonably expected to occur in methyl azide decomposition. Under collision-free conditions, photolysis of methyl azide has two likely primary photodissociation pathways corresponding to either $\mathrm{C}-\mathrm{N}$ bond breakage or $\mathrm{N}-\mathrm{N}$ bond breakage. In addition, it is unclear from the previous studies whether the spin-allowed molecular elimination channel will produce singlet methylnitrene as an intermediate or directly produce methanimine through a 
concerted 1,2 $\mathrm{H}$ atom shift isomerization. These mechanisms can be summarized as follows:

$$
\begin{aligned}
& \mathrm{CH}_{3} \mathrm{~N}_{3} \stackrel{h \nu}{\longrightarrow} \mathrm{CH}_{3}+\mathrm{N}_{3} \\
& \mathrm{CH}_{3} \mathrm{~N}_{3} \stackrel{h \nu}{\longrightarrow} \mathrm{CH}_{3} \mathrm{~N}+\mathrm{N}_{2} \\
& \mathrm{CH}_{3} \mathrm{~N}_{3} \stackrel{h \nu}{\longrightarrow} \mathrm{CH}_{2} \mathrm{NH}+\mathrm{N}_{2}
\end{aligned}
$$

These mechanisms will be referred to often during the analysis.

Reaction R1 will result in product fragments having $\mathrm{m} / z$ values of 15 and 42 . Both Reaction R2 and R3 will produce fragments with $\mathrm{m} / z$ values of 28 and 29 , so other characteristics will be required to distinguish between these mechanisms.

Some other primary dissociation pathways could be conceived of, though there is no prior or current experimental evidence of their existence. These would involve elimination of $\mathrm{N}, \mathrm{NH}$, or $\mathrm{NH}_{2}$ fragments. All of these channels are unlikely from an energetic stand point, and no direct evidence for these channels is observed.

\section{Singlet Methylnitrene Versus Methanimine}

Although the primary goal of this experiment was the identification of the radical channel R1 in the photodissociation of methyl azide, there is the opportunity to obtain additional information regarding a subject with conflicting opinions in the literature. Singlet methylnitrene would be the expected reaction product of the molecular elimination reaction R2. However, experimentally, this molecule has never been isolated and only the 1,2 $\mathrm{H}$ atom shift isomer methanimine has been observed. In the literature, several theorist have performed calculations to determine whether the singlet methylnitrene truly exists or if the dissociation and isomerization are a concerted process directly producing methanimine (i.e. Reaction R3). Several experimentalists have also used this mechanism to rationalize their observations in methyl azide decomposition.

In favor of the concerted reaction, Bock and Dammel in 1987 [7] and Shang et al. in 1995 [57] performed experiments which observed methanimine as a product of methyl azide decomposition. Their interpretation of the data left no evidence for an isolatable singlet nitrene intermediate. In 1999, Travers et al. [58] presented an electron photodetachment spectra which they claim show the singlet methylnitrene. Following up on this, Kemnitz et al. performed a calculation which they conclude "unequivocally predicts that singlet methylnitrene is an energy minimum with a barrier to rearrangement to methanimine that we estimate to be in the range of $2.5 \pm 1.0 \mathrm{kcal} / \mathrm{mol} . "$

Amid these conflicting reports, Arenas et al. also published the results of a theoretical calculation on this system (for thermal decomposition) in 1999 [1]. Despite finding that all the calculations converged on a direct formation of methanimine on the singlet surface as others have claimed, they profess a belief that "the concerted step is an artifact of the calculation." Furthermore, they state:

There is no other possible reaction path for this process than a nonconcerted mechanism, it being demonstrated from both the experimental and theoretical 
points of view that there is an exit barrier on the singlet surface which implies a transition state exists for such a reaction.

With these conflicting studies in the literature, my photofragment translational spectroscopy study can provide new experimental evidence in a collision free environment which may be able to differentiate between the concerted rearrangement (Reaction R3) or two step $\mathrm{N}_{2}$ elimination and subsequent isomerization of singlet methylnitrene (Reaction R2).

\subsubsection{Secondary Dissociation Pathways}

Secondary decomposition channels are also possible from energetically excited primary dissociation products. First, from previous $\mathrm{N}_{3}$ radical observations, it is possible for an internally excited $\mathrm{N}_{3}$ molecule to decompose into $\mathrm{N}$ and $\mathrm{N}_{2}$. Avoiding this secondary decomposition of cyclic $\mathrm{N}_{3}$ is one of the main reasons $\mathrm{CH}_{3} \mathrm{~N}_{3}$ was chosen for this experiment. From previous experiments, $\mathrm{CH}_{2} \mathrm{NH}$ has been observed to undergo secondary decomposition to produce HCN. Secondary decomposition of methanimine is interesting, as there is more than one mechanism which it can proceed by, which will produce slightly different observable intermediates and reaction products. First, $\mathrm{CH}_{2} \mathrm{NH}$ could undergo two subsequent $\mathrm{H}$-atom elimination processes. In this process, it is most likely that the $\mathrm{H}$ atom bonded to the nitrogen would be eliminated first, as it is a weaker bond than a $\mathrm{C}-\mathrm{H}$ bond. After losing a second $\mathrm{H}$ atom, hydrogen cyanide, $\mathrm{HCN}$, would be the resulting product. The other mechanistic possibility is a $1,1 \mathrm{H}_{2}$ elimination process where both of the carbon bonded hydrogen atoms form their own bond - producing an $\mathrm{H}_{2}$ molecule and leaving a hydrogen isocyanide, $\mathrm{CNH}$, fragment. These secondary channels can be summarized as follows:

$$
\begin{aligned}
\mathrm{N}_{3}^{*} & \longrightarrow \mathrm{N}+\mathrm{N}_{2} \\
\mathrm{CH}_{2} \mathrm{NH}^{*} & \longrightarrow \mathrm{HNC}+\mathrm{H}_{2} \\
\mathrm{CH}_{2} \mathrm{NH}^{*} & \longrightarrow \mathrm{CH}_{2} \mathrm{~N}+\mathrm{H} \longrightarrow \mathrm{HCN}+\mathrm{H}+\mathrm{H}
\end{aligned}
$$

It should be noted that the final end products of S3 are technically tertiary decomposition products, and that will have some bearing on the analysis. These secondary reactions produce some fragments which have the same mass as primary dissociation products $(m / z=28)$ which will provide some complication to the analysis. HCN and HNC are the only possible products which have a $m / z$ value of 27 , so observation of this mass will immediately imply Reactions S2 or S3 are occurring. Distinguishing between HNC and $\mathrm{HCN}$ will require some additional analysis. 


\section{$5.2248 \mathrm{~nm}$ Photolysis of Methyl Azide}

\subsubsection{Observed Photofragments}

In the $248 \mathrm{~nm}$ photolysis of methyl azide, fragments were observed and time of flight spectra recorded for the following $m / z$ values: 14, 15, 27, 28, and 29. Figure 5.1 shows representative time of flight spectra for each of these observed product fragments. The $m / z=29$ data has been scaled by 100 to make it more visible. No doubly charged ions were observed in the experiment, so no further mention of this possibility will be addressed. The possible fragments relevant to the expected dissociation channels corresponding to each of these masses are as follows:

$$
\begin{aligned}
& \boldsymbol{m} / \boldsymbol{z}=\mathbf{1 4}: \mathrm{N}, \mathrm{CH}_{2} \\
& \boldsymbol{m} / \boldsymbol{z}=\mathbf{1 5}: \mathrm{CH}_{3} \\
& \boldsymbol{m} / \boldsymbol{z}=\mathbf{2 7}: \mathrm{HCN}, \mathrm{HNC} \\
& \boldsymbol{m} / \boldsymbol{z}=\mathbf{2 8}: \mathrm{N}_{2}, \mathrm{CH}_{2} \mathrm{~N} \\
& \boldsymbol{m} / \boldsymbol{z}=\mathbf{2 9}: \mathrm{CH}_{2} \mathrm{NH}, \mathrm{CH}_{3} \mathrm{~N}
\end{aligned}
$$

\subsubsection{Methyl lodide Contamination}

I will begin the analysis with the $m / z=14$ and 15 photofragments as these are determined to be anomalous, and not involved in methyl azide photolysis. The first characteristic of these fragments is that the TOF spectra are nearly identical aside from different intensity. I examined whether the $m / z=15$ product fragment was formed from photolysis of a methyl iodide contaminant in the sample. This theory was tested by taking a previously reported center of mass translational energy distribution from the reported work by Barry and Gorry [4] for $248 \mathrm{~nm}$ photolysis of methyl iodide and using it with this instrument's experimental parameters to generate a forward convolution fit.

Figure 5.2 indicates the forward convolution fit generated from Barry and Gorry's $P\left(E_{T}\right)$ for the $m / z=14$ and 15 spectra as a red line. The spectrum has two narrow peaks, consistent with methyl fragments formed with momentum matched iodine atoms in two spin states $\left({ }^{2} \mathrm{P}_{3 / 2}\right.$ and $\left.{ }^{2} \mathrm{P}_{1 / 2}\right)$. The shape of the $m / z=14$ TOF spectrum is virtually identical to the $m / z=15$ including the two peaks, with lower intensity, and could be fit with the same $P\left(E_{T}\right)$ with a $m / z$ assumption of 15 . I therefore assigned this to be a $\mathrm{CH}_{2}$ fragment, likely formed by dissociative ionization of the $\mathrm{CH}_{3}$ fragment from methyl iodide, as the photon energy of the synchrotron radiation in these spectra is high enough $(17.05 \mathrm{eV})$ to exceed the dissociation threshold for $\mathrm{CH}_{3}$ (15.09 eV [11]). Therefore, I conclusively rule out the $m / z=14$ and 15 fragments as having any significance in the analysis of the methyl azide photolysis process. This leaves $\mathrm{m} / z$ values of 27,28 and 29 as the only product fragments from the photodissociation of methyl azide at $248 \mathrm{~nm}$. 

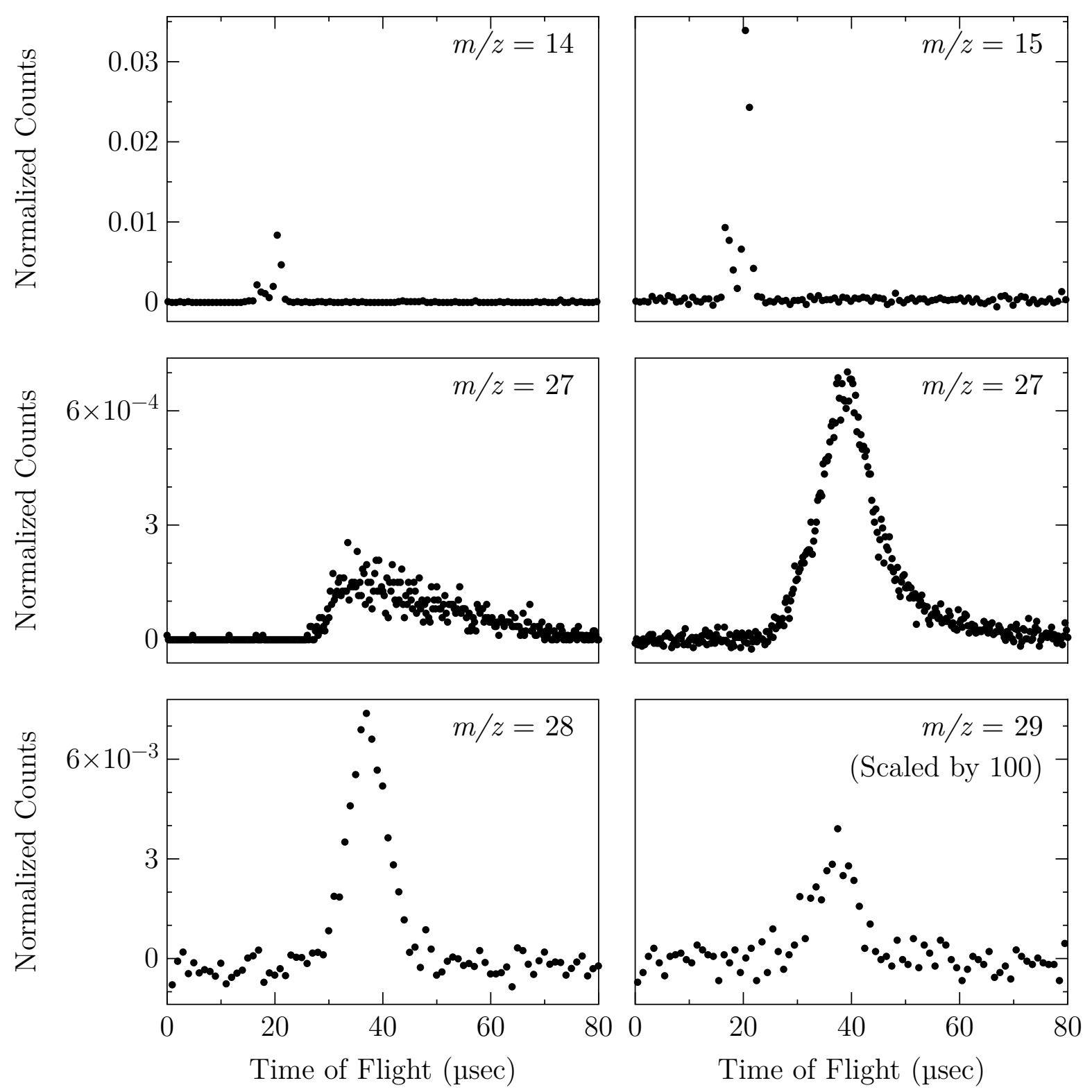

Figure 5.1: Representative time of flight spectra for each observed mass-to-charge ratio in the $248 \mathrm{~nm}$ photolysis of methyl azide. $\mathrm{m} / z=29$ has been scaled by 100 to be more visible. All spectra have been normalized to laser power, number of laser shots, and synchrotron radiation intensity. The detector angle was $30^{\circ}$ from the molecular beam in all displayed spectra. The photoionization energy used to detect each fragment is discussed in the analysis of each individual spectrum. 

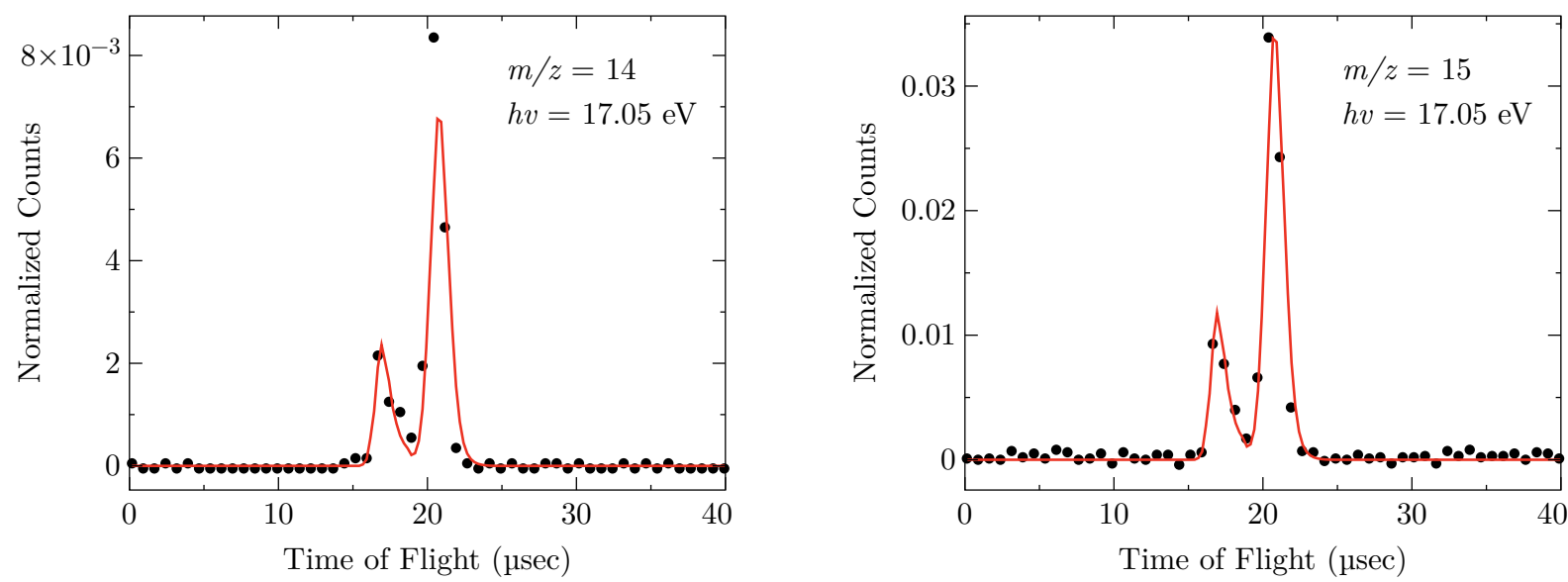

Figure 5.2: Time of flight spectra for $\mathrm{m} / z=14$ and 15 in the $248 \mathrm{~nm}$ photolysis of methyl azide. The solid black points are the experimental data and the red line is the result of the forward convolution fitting described in the text. Spectra have been normalized for laser power, number of laser shots, and synchrotron radiation intensity. The laboratory angle was $30^{\circ}$ and the photoionization energy was $17.05 \mathrm{eV}$ in both spectra. Left: $m / z=14$. Right: $m / z=15$.

\subsubsection{Overview of Thermodynamics of $248 \mathrm{~nm}$ Photolysis}

A complete understanding and assignment which distinguishes between Reactions R2 and $\mathrm{R} 3$ requires an analysis of the relevant energetics of these dissociation pathways. Figure 5.3 shows select points relevant to the product fragments observed on the lowest singlet and triplet potential energy surfaces of methyl azide using ab initio calculation results from M. Nguyen et al. for the heats of formations and exit barriers for each of these processes [47].

The singlet potential energy surface is connected by a dashed line and the triplet surface is connected by a dotted line. The maximum available energy after photolysis by a single $248 \mathrm{~nm}$ photon is represented by the horizontal dashed line at $187.5 \mathrm{kcal} / \mathrm{mol}$ and the energy of a single photon is represented by the upward pointing arrow labeled $h \nu$. Additionally, there is a point which represents the maximum translational energy release determined by forward convolution fitting for the $m / z=28$ and 29 reaction channel, with the full $P\left(E_{T}\right)$ plotted beside it for reference. I will return to this figure as necessary throughout the analysis.

\subsubsection{Assignment of Primary Dissociation Channel}

Having ruled out any observation of $m / z=15$ from the methyl azide photolysis and no observable signal at $m / z=42$ (despite considerable effort searching for it), I find no evidence of the radical channel, Reaction R1, occurring in the $248 \mathrm{~nm}$ photolysis process.

The molecular elimination channels are expected to produce fragments at $m / z$ values of 29 and 28, and also 27 through secondary dissociation processes. The forward convolution fitted time of flight spectra for fragments of $m / z=29$ and 28 can be seen in Figure 5.4. 


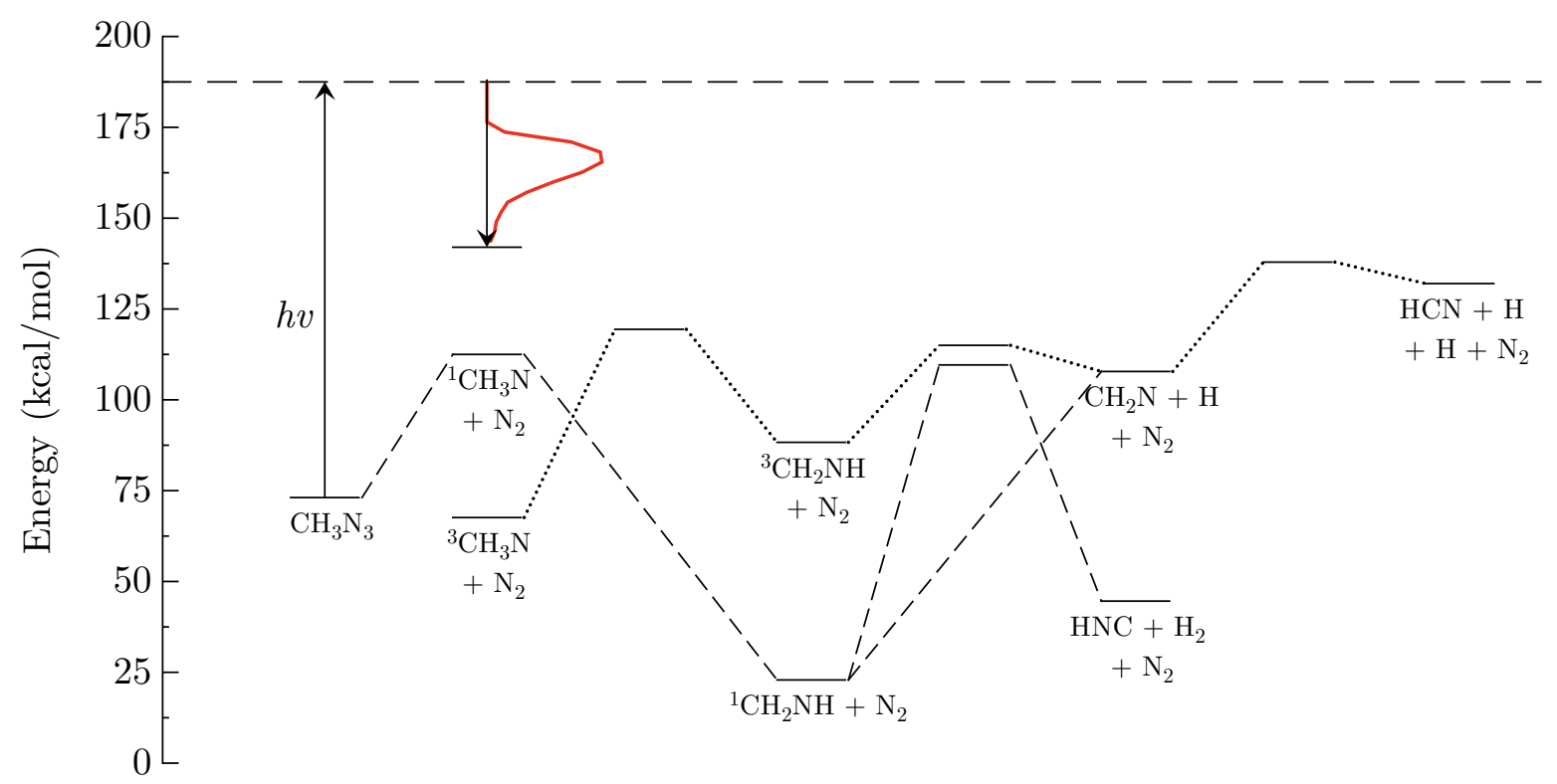

Figure 5.3: Energy diagram relevant to the observed $248 \mathrm{~nm}$ photolysis channels. Energy values for each point taken from M. Nguyen in Reference [47]. The singlet potential energy surface is connected by dashed lines. The triplet potential energy surface is connected by dotted lines. The center of mass translational energy release probability for the primary dissociation from Figure 5.5 is shown by a solid red line.

The photoionization energy for these spectra is $17.05 \mathrm{eV}$, high enough to ionize both $\mathrm{N}_{2}$ (15.58 eV [35]) and $\mathrm{CH}_{2} \mathrm{NH}(9.97 \mathrm{eV}[7])$.

The intensity has been normalized for laser power, number of laser shots, and synchrotron radiation intensity. A constant background signal has been subtracted from each data point as well. As explained in Chapter 3, two fragments whose time of flight spectra can be fit through forward convolution fitting using one $P\left(E_{T}\right)$ are momentum matched and must originate from the same dissociation pathway. The solid red line shown in Figure 5.4 indicates the calculated forward convolution fitting for the $P\left(E_{T}\right)$ in Figure 5.5.

These two fragments are momentum matched partners and therefore these products should be assigned to either the reaction channel R2 or R3. The $P\left(E_{T}\right)$ in Figure 5.5 shows an average translational energy of around $25 \mathrm{kcal} / \mathrm{mol}$. The maximum possible translational energy determined by the heats of formation of each product for the two reaction channels R2 and R3 from Figure 5.3 is indicated as well. If R3 is a significant reaction pathway, one would have to rationalize why only around $12 \%$ of the available energy appears in translation. A more likely explanation is that the initial photodissociation proceeds through pathway $\mathrm{R} 2$ forming ${ }^{1} \mathrm{CH}_{3} \mathrm{~N}$. This fragment then has enough internal energy to later isomerize to $\mathrm{CH}_{2} \mathrm{NH}$ on a short time scale, which will result in a fragment with around $120 \mathrm{kcal} / \mathrm{mol}$ of internal energy. This highly internally excited molecule can then undergo further unimolecular dissociation reactions, producing the $\mathrm{m} / z=27$ frag- 

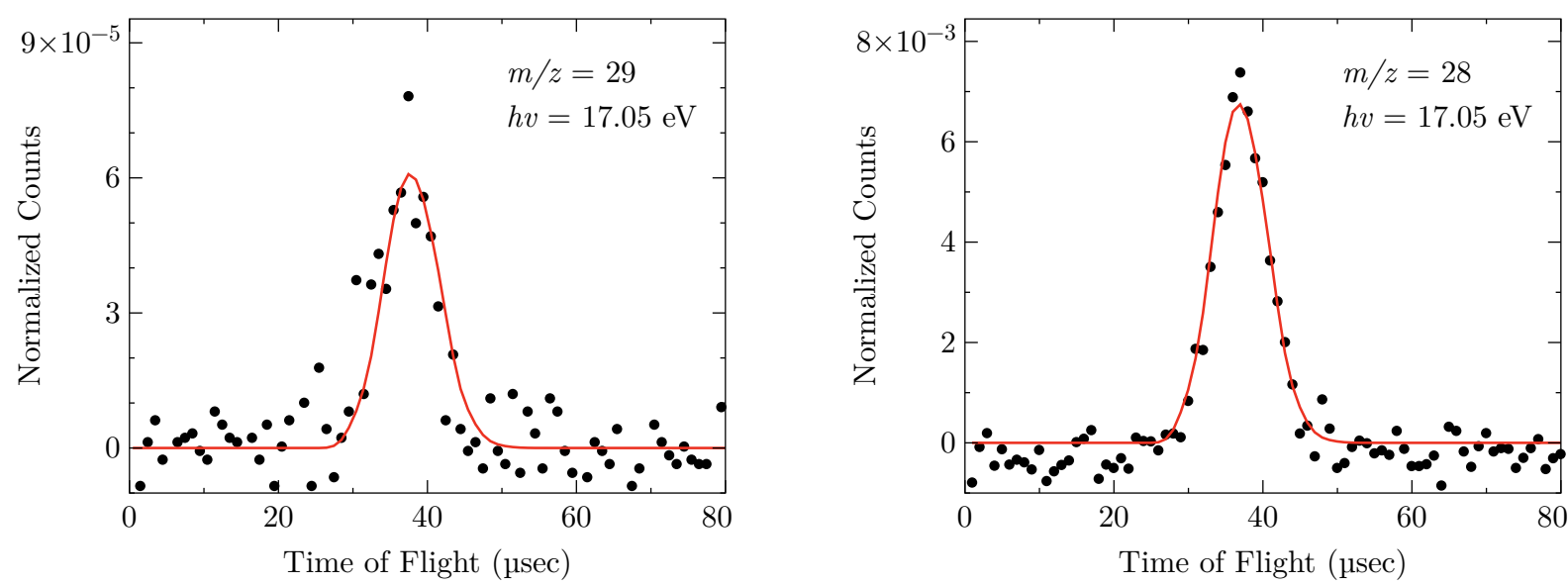

Figure 5.4: Time of flight spectra for $\mathrm{m} / z=29$ and 28 in the $248 \mathrm{~nm}$ photolysis of methyl azide. The solid black points are the experimental data and the red line is the result of the forward convolution fit for the $P\left(E_{\mathrm{T}}\right)$ in Figure 5.5. Spectra have been normalized for laser power, number of laser shots, and synchrotron radiation intensity. The laboratory angle was $30^{\circ}$ and the photoionization energy was $17.05 \mathrm{eV}$ in both spectra. Left: $m / z=29$. Right: $m / z=28$.

ments observed. This hypothesis is supported by the extremely low signal of the $\mathrm{m} / z=29$ fragment relative to the partner $\mathrm{N}_{2}$ fragment. The observation that the product fragment peaks appear momentum matched despite significant unimolecular dissociation of one product suggests that the dissociation probability is independent of the translational energy for the $m / z=29$ fragment. In other words, all $m / z=29$ fragments have sufficient internal energy to undergo further dissociation.

Other possibilities which could lead to similar results should also be addressed. The similar shape of the peaks along with the large difference in the intensity of the spectra may suggest the observed $m / z=29$ fragment is due to the ${ }^{15} \mathrm{~N}-{ }^{14} \mathrm{~N}$ molecular nitrogen isotope. This theory can effectively be ruled out by the photoionization range for the $m / z=29$ fragment. This fragment can be observed as much as $3.5 \mathrm{eV}$ below the ionization potential of molecular nitrogen.

Another reaction channel that could conceivably occur is a $\mathrm{N}_{2} \mathrm{H}$ elimination mechanism, which would also produce $m / z=28$ and 29 as the primary reaction product fragments. I rule out this reaction pathway for several reasons. First, $\mathrm{N}_{2} \mathrm{H}$ is a highly unstable species with a very short lifetime (less than 6 nsec) and would therefore not even be detectable in the time frame of the experiment [60]. The $\mathrm{N}_{2} \mathrm{H}$ molecule also has a very low ionization potential of $7.8 \mathrm{eV}$. When the photoionization energy of the synchrotron is tuned above $7.8 \mathrm{eV}$ but below the ionization potential of $\mathrm{CH}_{2} \mathrm{NH}(9.97 \mathrm{eV}[7])$ the $m / z=29$ signal is not observed. There is then no experimental evidence that the $m / z=29$ fragment should be attributed to $\mathrm{N}_{2} \mathrm{H}$. Finally, it should be noted that $\mathrm{N}_{2}$ cannot be formed in an excited state in this experiment. There is not enough energy available in a single $248 \mathrm{~nm}$ photon to produce the lowest level excited electronic state of $\mathrm{N}_{2}$ in the dissociation. 


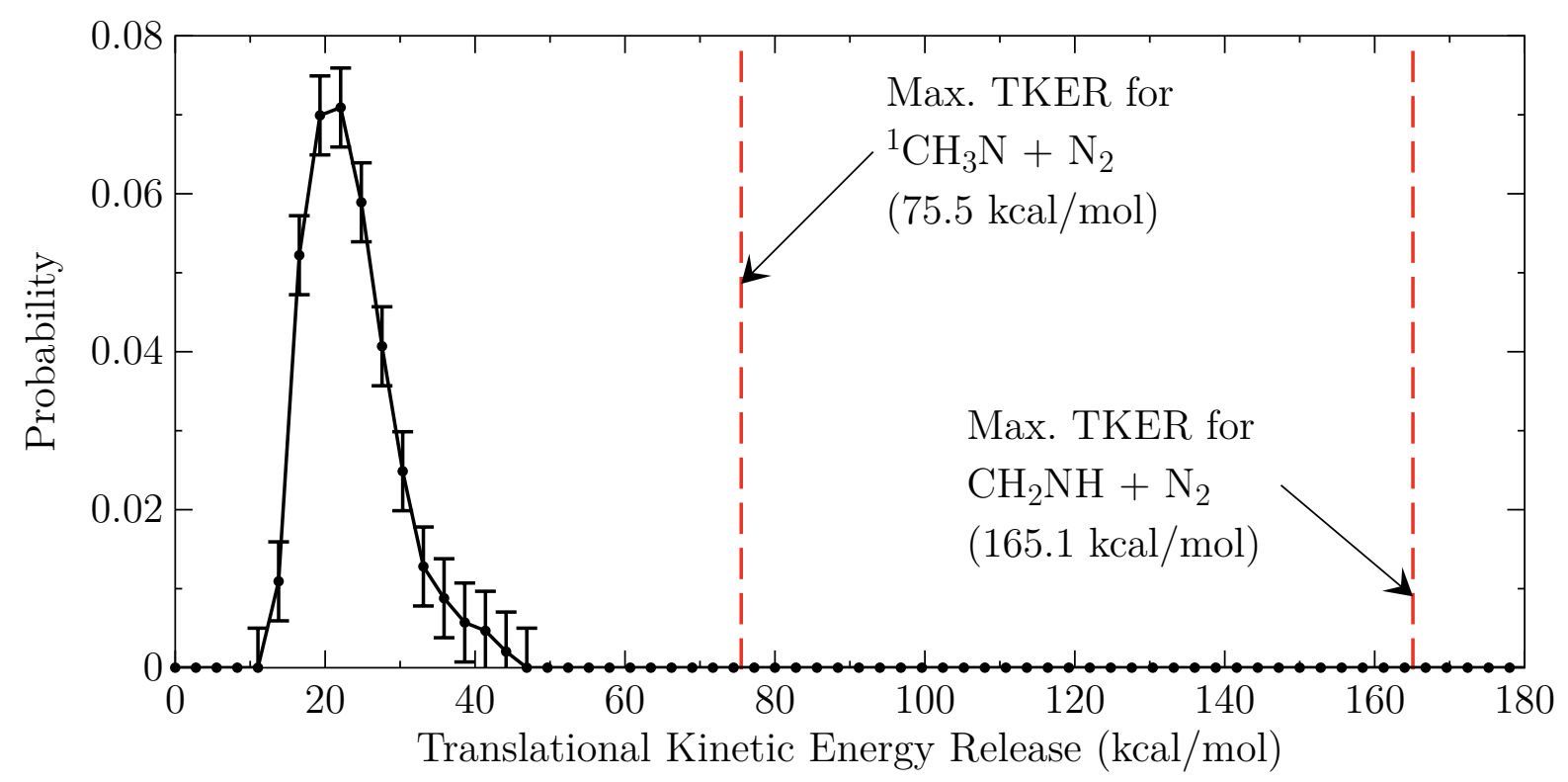

Figure 5.5: Center of mass translational energy release probability, $P\left(E_{\mathrm{T}}\right)$, used to fit $m / z=28$ and 29 fragments from the primary dissociation process in $248 \mathrm{~nm}$ photolysis. The labeled dotted lines represent the maximum energy release allowed as determined from the heats of formation and energy barriers in reference [47] and shown in Figure 5.3.

To summarize the assignment of the primary dissociation channels: Reaction R2 is the primary dissociation pathway, but the ${ }^{1} \mathrm{CH}_{3} \mathrm{~N}$ fragment produced rapidly isomerizes to $\mathrm{CH}_{2} \mathrm{NH}$. The $m / z=28$ and 29 spectra then belong to the product fragments $\mathrm{N}_{2}$ and $\mathrm{CH}_{2} \mathrm{NH}$ respectively. The $\mathrm{CH}_{2} \mathrm{NH}$ fragment contains a significant amount of internal energy in this mechanism and undergoes further secondary unimolecular dissociation into additional products accounting for the very low observed signal of this molecule.

\subsubsection{Assignment of Secondary Dissociation Channels}

As demonstrated in the previous section, the $\mathrm{CH}_{2} \mathrm{NH}$ fragment produced in this experiment has a large amount of internal energy from the isomerization and a low observed signal, leading to the conclusion that unimolecular dissociation is likely occurring. This leads to the topic of the observed $m / z=27$ fragment. No primary dissociation pathway can produce a fragment with that $m / z$ value, so it must be produced in a secondary dissociation process. The highly excited $\mathrm{CH}_{2} \mathrm{NH}$ fragment must lose two $\mathrm{H}$ atoms to produce the $m / z=27$ fragment which then can be assigned to either HCN or HNC via secondary reaction channels $\mathrm{S} 2$ or $\mathrm{S} 3$. The ability to tune the synchrotron radiation photoionization energy is key to distinguishing between these two possibilities.

The TOF spectra for $m / z=27$ taken with a high and low photoionization energy are shown in Figure 5.6. The right panel shows the TOF with a high photoionization energy 

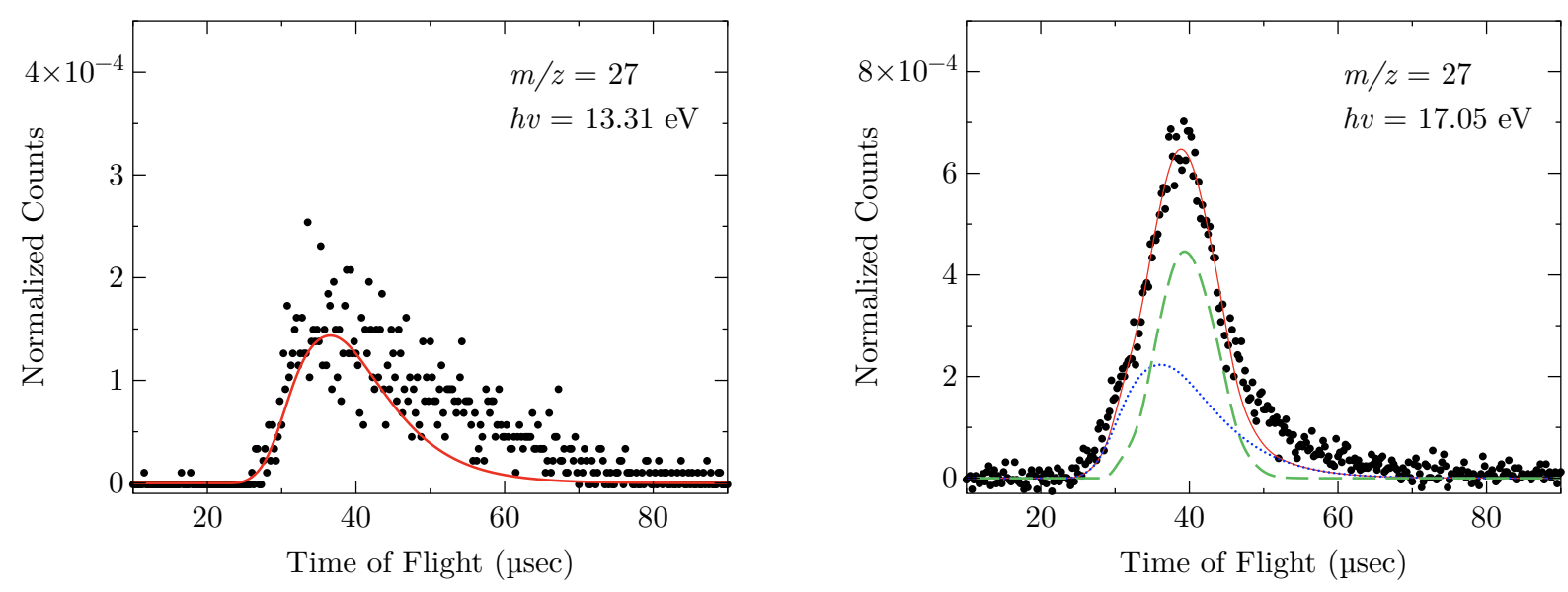

Figure 5.6: Time of flight spectra for $m / z=27$ in the $248 \mathrm{~nm}$ photolysis of methyl azide. The left panel shows data taken with photoionization energy of $13.31 \mathrm{eV}$. The right panel shows data taken with photoionization energy of $17.05 \mathrm{eV}$. The solid black points are the experimental data. The red line in the left panel is the result of a secondary dissociation process forward convolution fit for the $P\left(E_{\mathrm{T}}\right)$ in Figure 5.7. In the right panel, the blue curve is the same fit as the red line in the left panel. The green curve is the result of a secondary dissociation fit whose translational energy peaks at near zero. The red line in the right panel is the sum of the blue and green curves. Spectra have been normalized for laser power, number of laser shots, and synchrotron radiation intensity. The laboratory angle was $30^{\circ}$ in both spectra.

$(17.05 \mathrm{eV})$ and the left panel shows the TOF with a low photoionization energy $(13.31 \mathrm{eV})$. There appear to be two components to the high photoionization energy spectrum - a slower, more intense peak in the middle of a less intense, broader, fast peak. This broad, fast peak is the only component of the low ionization energy spectra. HCN has an ionization potential of $13.6 \mathrm{eV}$ [25], so it should not be observable in the left panel. HNC, on the other hand, has a lower ionization potential of $12.5 \mathrm{eV} \mathrm{[5]} \mathrm{and} \mathrm{could} \mathrm{be} \mathrm{observed} \mathrm{at} \mathrm{this} \mathrm{photoionization}$ energy. From these characteristics, the fast component should be HNC produced through secondary reaction S2 and the slower peak, observable at high ionization energies, should be assigned to HCN produced through S3.

The forward convolution fits to the data and the resulting center of mass translational energy release probabilities give more confidence that this assignment is correct. As these are secondary dissociation processes (technically tertiary in the case of the final HCN fragment) I use ANALMAX for the analysis. To fit the single component in the left panel, the primary $P\left(E_{T}\right)$ shown in Figure 5.5 was used along with a secondary $P\left(E_{T}\right)$ shown in Figure 5.7.

The resulting secondary $P\left(E_{T}\right)$ shows a rather large translational energy release for this process (up to $30 \mathrm{kcal} / \mathrm{mol}$ ). This result is consistent with the scenario proposed by reaction S2 where a closed shell reactant forms two closed shell products in close proximity 


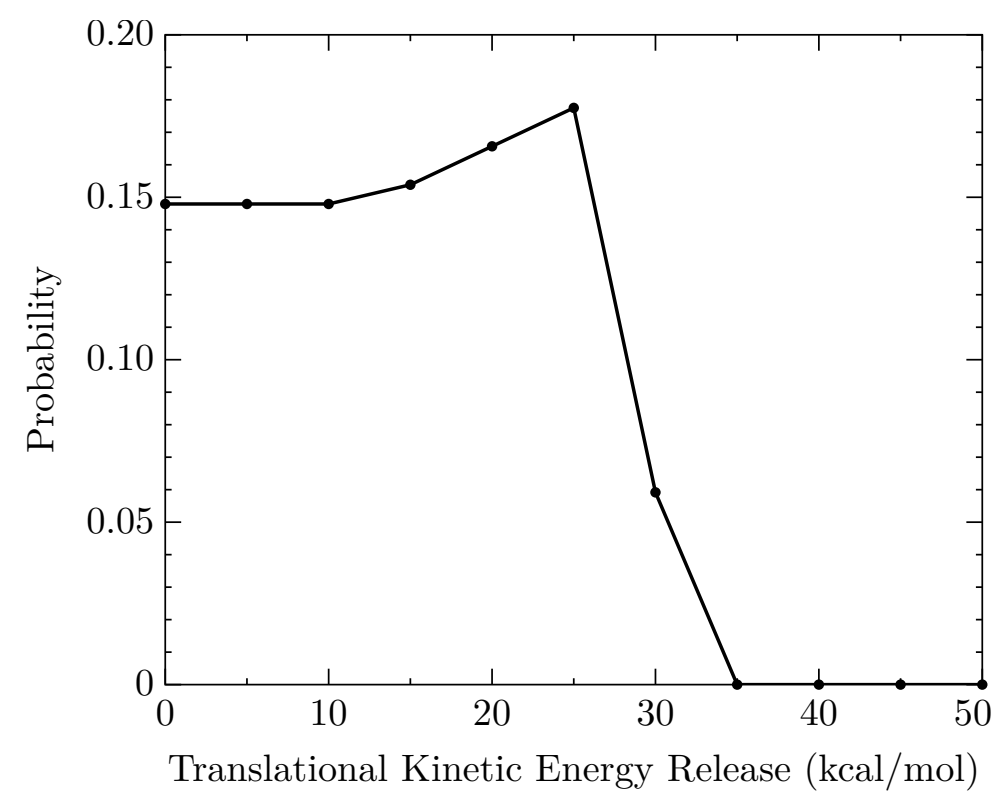

Figure 5.7: Center of mass translational energy release probability, $P\left(E_{\mathrm{T}}\right)$, used to fit the low ionization energy component of the $m / z=27$ fragment from secondary dissociation in the $248 \mathrm{~nm}$ photolysis.

to each other.

The fit to the additional slow component in the upper panel is a bit trickier and requires some assumptions to fit. ANALMAX is not capable of modeling tertiary dissociation reactions directly. However, in this case, I expect both dissociation steps to have very low translational energy releases due to the simple bond rupture process producing $\mathrm{H}$ atoms, which have low mass. My use of AnALMAX to fit this peak essentially relies on the assumption that the intermediate step has a translational energy release distribution which sharply peaks near zero. This allows me to assume the primary $P\left(E_{T}\right)$ is the most important factor in arriving at the tertiary energy distribution, and I can model the tertiary process as a secondary dissociation in ANALMAX. The fact that this peak could be fit with little modification to the primary $P\left(E_{T}\right)$ is consistent with my assignment of the peak to HCN being produced through two low energy release simple bond rupture processes.

One additional piece of evidence for the sequential $\mathrm{H}$ atom loss mechanism can be found in a low ionization energy $m / z=28$ TOF spectrum. Figure 5.8 shows an example of the $m / z=28$ data when the synchrotron radiation is tuned below the ionization threshold for $\mathrm{N}_{2}$. The red line forward convolution fit comes from a near zero secondary dissociation $P\left(E_{T}\right)$ while the blue dotted line is the position of the primary $\mathrm{N}_{2}$ product fragment. It is clear that the low ionization energy product arrives slightly earlier in time than the $\mathrm{N}_{2}$ fragment, implying it is a different species. It is reasonable to expect that if the sequential $\mathrm{H}$ atom loss mechanism occurs and has some energetic barrier to the second $\mathrm{H}$ atom loss, one would be able to detect some of the intermediate species, $\mathrm{CH}_{2} \mathrm{~N}$, which does not undergo the second $\mathrm{H}$ atom loss step. Indeed, something is detected in the photoionization 


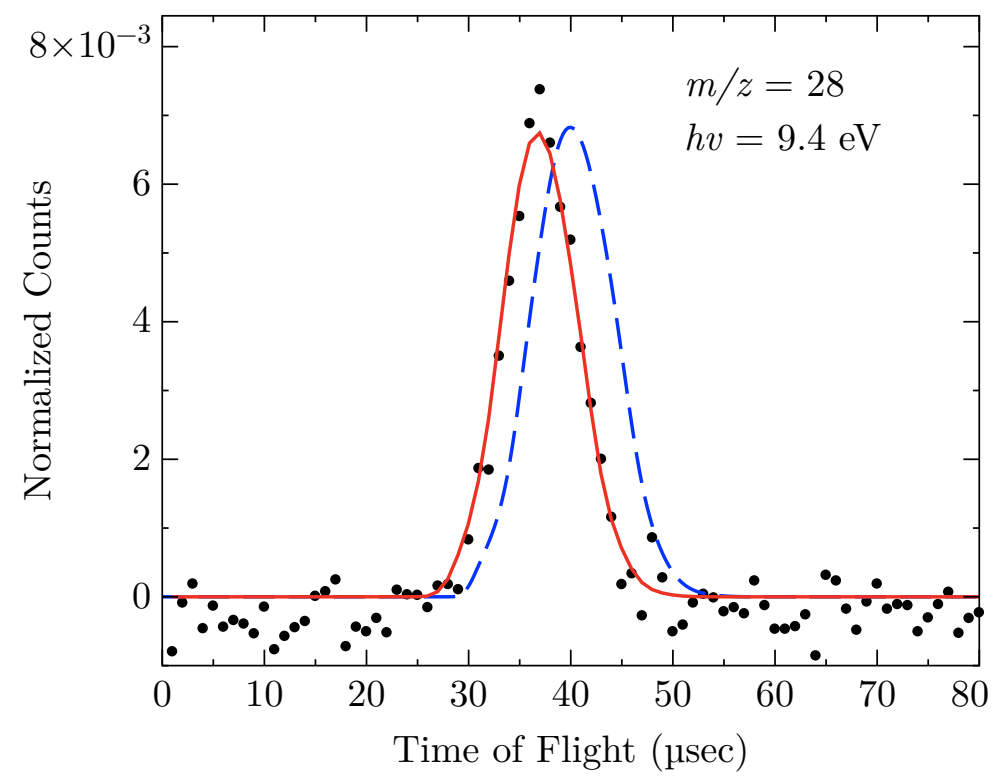

Figure 5.8: Time of flight spectra for $\mathrm{m} / z=28$ with $9.4 \mathrm{eV}$ ionization energy in $248 \mathrm{~nm}$ photolysis. The solid red line is the secondary forward convolution fit to the data for the an essentially zero translational energy release secondary dissociation. The blue dotted line shows the slightly offset position of the high ionization energy $m / z=28$ fragment. Spectra have been normalized for laser power, number of laser shots, and synchrotron radiation intensity. The laboratory angle was $30^{\circ}$.

range expected for $\mathrm{CH}_{2} \mathrm{~N}$ (above $9.4 \mathrm{eV}$ [46]). However, I cannot definitively conclude that this fragment is the intermediate in the $\mathrm{H}$ atom loss mechanism. As already shown, $\mathrm{CH}_{2} \mathrm{NH}$ should be formed with very high internal energies. It is then also possible that the $\mathrm{CH}_{2} \mathrm{~N}$ is formed in dissociative ionization of methanimine from the synchrotron radiation. There is not much experimental data I can use to distinguish between these possibilities. The presence of a lower ionization energy fragment at $m / z=28$ is expected from my interpretation of the data.

\subsubsection{Evidence of Singlet Methylnitrene}

As I concluded in Section 5.2.4, I find that the initial formation of singlet methylnitrene is more likely than a concerted elimination/isomerization mechanism producing methanimine directly. Examining it in the context of the available energy, it seems unlikely that Reaction R2 would lead to such a low fraction of energy going into translation. The simultaneous elimination and rearrangement would produce molecular nitrogen and methanimine (two closed shell molecules) within bonding distance of each other. There should be a strong repulsion between the product fragments in this situation and a substantially larger translational energy release. 
Examples of previous experimental results of photofragment translational spectroscopy for reactions producing two closed shell products can be found in References [34] and [62]. In Reference [62], HONO elimination from nitroethane and 2-nitropropane saw $70 \%$ of the exit barrier energy go into translational energy. Similarly, in Reference [34], molecular elimination from ethylacetate to produce acetic acid and ethylene saw more than $50 \%$ of the exit barrier energy in translation. The methyl azide potential energy surface has the exit barrier producing methanimine of more than $90 \mathrm{kcal} / \mathrm{mol}$. It is difficult to understand why only $28 \%$ of the exit barrier would go into translation. The simpler explanation is that the initial product is singlet methylnitrene which has more than $120 \mathrm{kcal} / \mathrm{mol}$ of energy held in the potential energy of its structure.

These two mechanisms can also be analyzed in the context of the "soft product impulse approximation" which approximates the initial release by forcing the atoms sharing the dissociating bond and the resulting photofragments to obey conservation of linear momentum [50]. The ratio between $E_{T}$ and $E_{a v a}$ is calculated to be 0.49 in this approximation. From the energy diagram in Figure 5.3, for Reaction R2 this gives an approximation for the expected translational energy of $37 \mathrm{kcal} / \mathrm{mol}$. For Reaction R3, this value is $81 \mathrm{kcal} / \mathrm{mol}$. The experimentally observed average translational energy of this reaction determined from Figure 5.5 is $29 \mathrm{kcal} / \mathrm{mol}$. This model favors the singlet methylnitrene pathway when compared to experimental results.

\subsubsection{Comment on Role of Triplet Potential Energy Surface}

The role of the triplet potential energy surface in this experiment is uncertain. It is not needed to provide a thorough explanation of the observed fragments and their energy distributions. There are some previously performed photolysis experiments which favor the singlet surface and some which favor the triplet surface. In $\mathrm{HN}_{3}$, the simplest azide, which has been the subject of a number of photochemical experiments [15, 66], the singlet channel is strongly favored when there is enough energy for spin-allowed dissociation [66]. Another experimental analog can be seen in the reports on dissociation of ketene [45]. In favor of the triplet surface, triplet methylnitrene was observed in methyl azide photolysis experiments in References [43, 65]. As triplet methylnitrene is a molecule with previous experimental observation, it may be a simpler explanation for our data than the singlet state, which has not been conclusively isolated. However, the experiment which produced the triplet state was not performed under collision-free conditions, thus the role collisions play in intersystem crossing is unclear and the triplet may not necessarily be produced under collision-free conditions. Indeed, if the dissociation was producing triplet methylnitrene in substantial quantities, I would expect to see a larger translational energy release in the secondary and tertiary dissociation pathways. As can be seen in the energy diagram of Figure 5.3, from Nguyen's calculations, there is an energy barrier to secondary reaction S3 on the triplet surface. However, as already discussed, I determine there to be little to no energy release in this channel, supporting the interpretation that this dissociation is occurring on the singlet potential energy surface. While it is reasonable to think the triplet state can play a role in this experiment, there is no clear evidence of it and it is not 
needed to provide a satisfactory interpretation of the data.

\section{$5.3193 \mathrm{~nm}$ Photolysis of Methyl Azide}

\subsubsection{Observed Photofragments}

In the $193 \mathrm{~nm}$ photolysis of methyl azide, fragments were detectable and time of flight data was recorded for the following values of $\mathrm{m} / z: 14,15,27,28,29$ and 42 . Figure 5.9 shows a representative time of flight spectrum for each of these fragments at a laboratory angle of $30^{\circ}$. A summary of the possible assignment of these product fragments, analogous to that given for the $248 \mathrm{~nm}$ process is:

$$
\begin{aligned}
& \boldsymbol{m} / \boldsymbol{z}=\mathbf{1 4}: \mathrm{N}, \mathrm{CH}_{2} \\
& \boldsymbol{m} / \boldsymbol{z}=\mathbf{1 5}: \mathrm{CH}_{3} \\
& \boldsymbol{m} / \boldsymbol{z}=\mathbf{2 7}: \mathrm{HCN}, \mathrm{HNC} \\
& \boldsymbol{m} / \boldsymbol{z}=\mathbf{2 8}: \mathrm{N}_{2}, \mathrm{CH}_{2} \mathrm{~N} \\
& \boldsymbol{m} / \boldsymbol{z}=\mathbf{2 9}: \mathrm{CH}_{2} \mathrm{NH}, \mathrm{CH}_{3} \mathrm{~N} \\
& \boldsymbol{m} / \boldsymbol{z}=\mathbf{4 2 :} \mathrm{N}_{3}
\end{aligned}
$$

This is identical to the results of the $248 \mathrm{~nm}$ photolysis, but with the important addition of an observable $m / z=42$ product fragment. The presence of this product immediately suggests there should be a momentum matched $m / z=15$ partner from Reaction R1. In the $248 \mathrm{~nm}$ experiment, I demonstrated that the entire $\mathrm{m} / z=15$ signal originated from methyl iodide contamination in my sample. In order to again determine if any or all of the signal at $193 \mathrm{~nm}$ is due to the $\mathrm{CH}_{3} \mathrm{I}$ impurity, I rely on published data by Continetti et al. [12] for $\mathrm{CH}_{3} \mathrm{I}$ photolysis at $193 \mathrm{~nm}$. While they did not publish the translational energy release probability directly, by using their experimental conditions, I use PHOTRAN to simulate a fit to the same TOF data points given in the publication and then use that $P\left(E_{T}\right)$ to simulate how it would appear under my own experimental conditions. Figure 5.10 shows the time of flight spectrum of the $m / z=15$ product fragment recorded in this experiment and the fit produced using this method. There are two distinct peaks in the TOF - a sharp, fast peak and a slower, broad peak. The results are in good agreement with the sharp, fast peak in the spectrum. This can be seen as the solid red line in Figure 5.10. This peak is well resolved from the slower peak. With no indication that the $\mathrm{CH}_{3} \mathrm{I}$ contributes to the slow peak, I conclude this slow peak to be a product of the photolysis of methyl azide, and thus the impurity's contribution to the TOF spectra should not significantly affect my analysis of the data. 

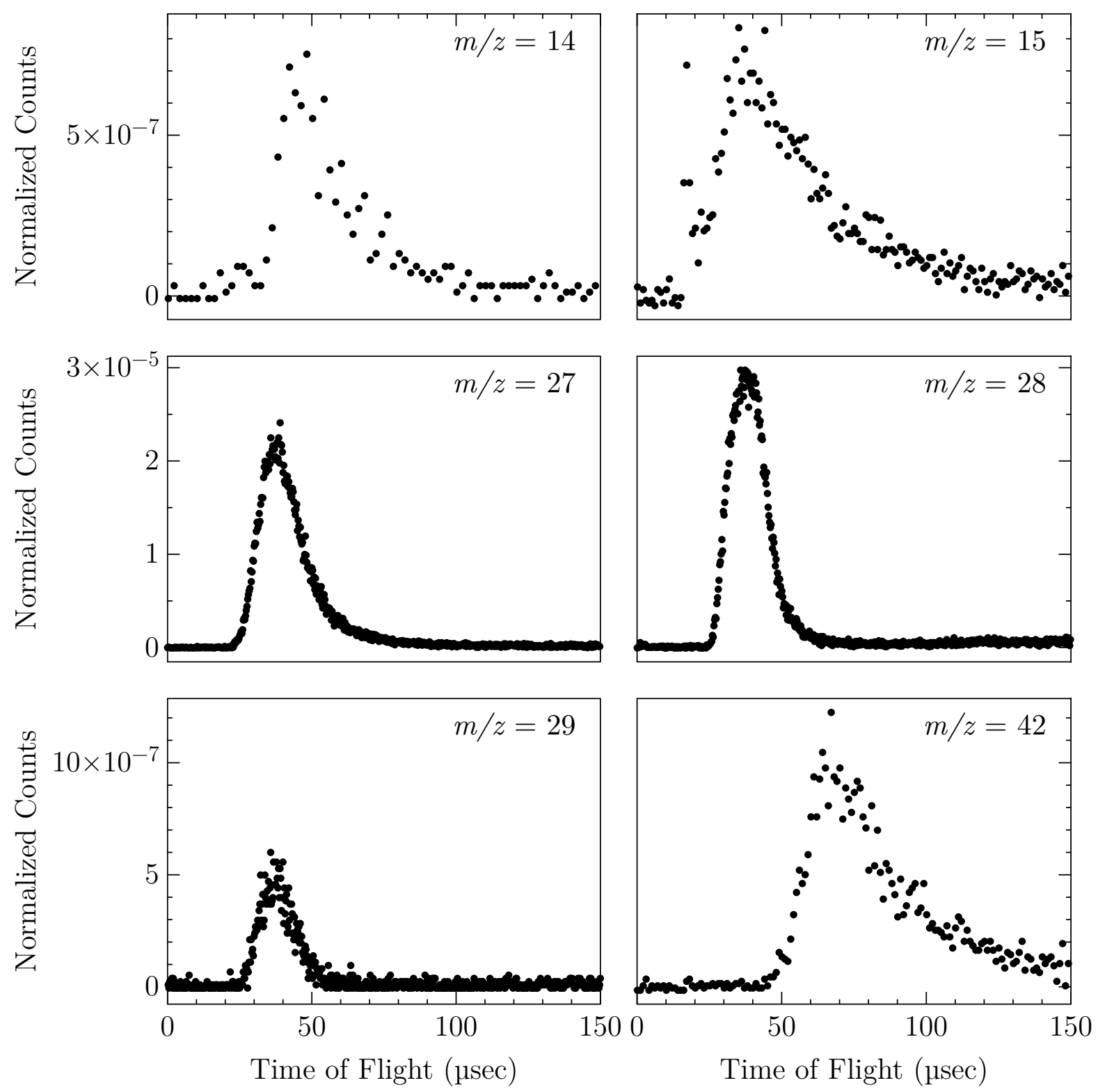

Figure 5.9: Representative time of flight spectra for each observed mass-to-charge ratio in the $193 \mathrm{~nm}$ photolysis of methyl azide. All spectra have been normalized to laser power, number of laser shots, and synchrotron radiation intensity. The detector angle was $30^{\circ}$ from the molecular beam in all displayed spectra. The photoionization energy used to detect each fragment is discussed in the analysis of each individual spectrum. 


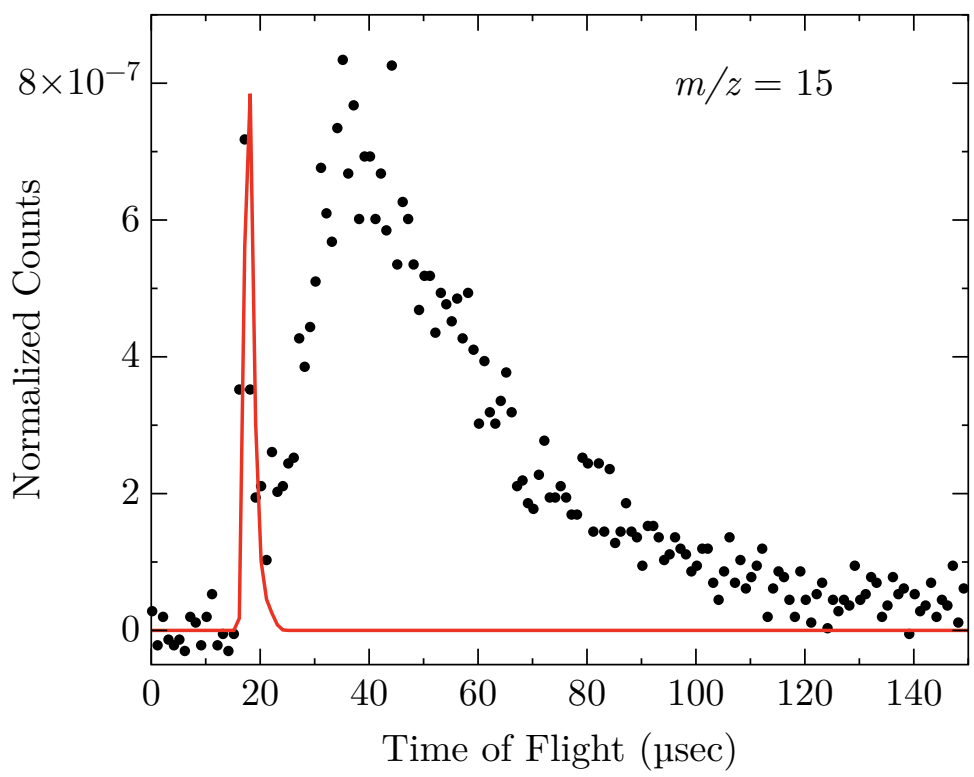

Figure 5.10: Time of flight spectra for $\mathrm{m} / z=15$ in $193 \mathrm{~nm}$ photolysis. The solid black points are the experimental data. The solid red line is the forward convolution fit resulting from the $P\left(E_{T}\right)$ derived from Reference [12] for $193 \mathrm{~nm}$ photolysis of $\mathrm{CH}_{3}$ I. Spectra have been normalized for laser power, number of laser shots, and synchrotron radiation intensity. The laboratory angle was $30^{\circ}$.

\subsubsection{Overview of Thermodynamics of $193 \mathrm{~nm}$ Photolysis}

A diagram of the energetics relevant to the radical dissociation channel R1 at $193 \mathrm{~nm}$ photolysis wavelength is shown in Figure 5.11. The data in this figure is based on a compilation of published thermodynamic values from various literature sources. Critical to my analysis is the heat of formation of methyl azide. This value has not been determined very accurately in experimental work and several different values have been reported from both experimental and theoretical sources. Recent experimental work by Alfredo QuintoHernandez and theoretical calculations by Dr. Ricardo Mata provide a new estimate of the $\mathrm{CH}_{3}-\mathrm{N}_{3}$ bond dissociation energy of $70.0 \mathrm{kcal} / \mathrm{mol}$ [49]. This value is around $4 \mathrm{kcal} / \mathrm{mol}$ higher in energy than what was considered in the analysis of this data when it was first published in 2008 [39]. For the current analysis, I will be using the recently updated value, as it is likely the most accurately determined value thus far. For the heat of formation of $\mathrm{CH}_{3}$, I referenced Ruscic et al. for the value of $35.85 \mathrm{kcal} / \mathrm{mol}$ [51]. For linear $\mathrm{N}_{3}$, I find the value of $113.7 \mathrm{kcal} / \mathrm{mol}$ from the work of Continetti et al. [13, 14]. Cyclic $\mathrm{N}_{3}$ ring formation is expected to cost $30.3 \mathrm{kcal} / \mathrm{mol}$ of energy over the linear form [68], or alternatively, there is an experimental result which estimates the heat of formation of cyclic $\mathrm{N}_{3}$ at $142 \mathrm{kcal} / \mathrm{mol}$ [55]. The barrier heights and energy levels for unimolecular dissociation of $\mathrm{N}_{3}$, for both linear and cyclic forms, are obtained from theoretical calculations by K. Morokuma et al. [68].

The thermodynamics of reactions R2 and R3 are similar to the values given in Figure 5.3. 


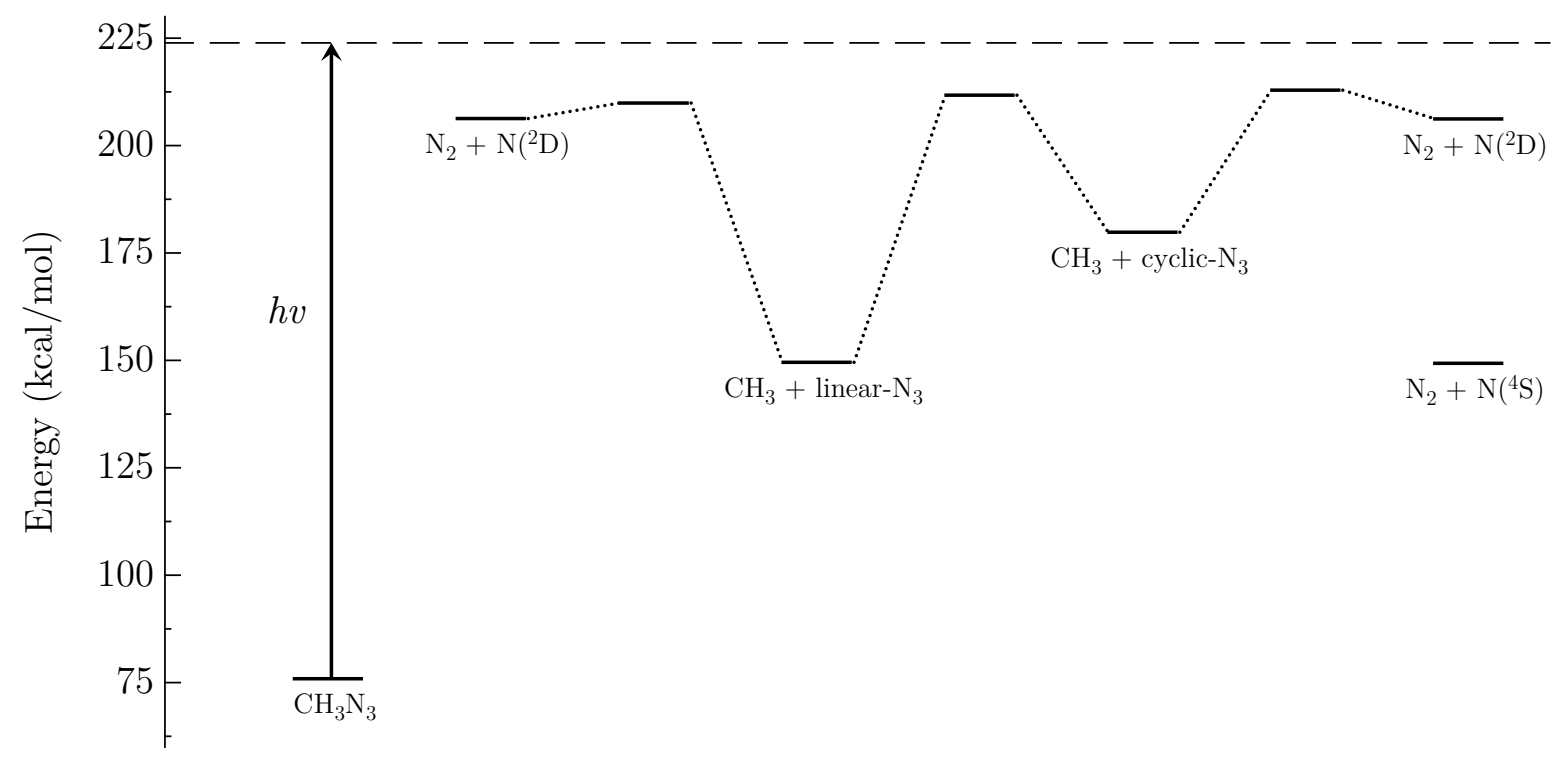

Figure 5.11: Energy diagram relevant to the additional $\mathrm{N}_{3}$ channels observed in $193 \mathrm{~nm}$ photolysis of methyl azide. Energy values compiled from several references $[13,14,51,68]$.

The only difference is that there is a higher total energy available to the system.

\subsubsection{Assignment of Primary Dissociation Channels}

\section{Radical Channel}

Figure 5.12 shows the time of flight spectra for the two fragments which should originate from reaction channel $\mathrm{R} 1, \mathrm{~m} / z=15$ and 42 , corresponding to $\mathrm{CH}_{3}$ and $\mathrm{N}_{3}$ respectively. The solid red lines through the data are the fits created through forward convolution fitting in PHOTRAN. The dashed blue line is the methyl iodide contribution which was fit in a previous section. The translational energy release probability distributions were extracted and can be seen in Figure 5.13. The $P\left(E_{T}\right)$ fitting the $m / z=15$ data is shown as open circles while the $P\left(E_{T}\right)$ fitting the $m / z=42$ data is shown as filled circles.

Up until now, I have placed significant emphasis on the characteristic of momentum matched fragments having matching translational energy release probabilities if they come from the same dissociation channel. Here, although the distributions are not identical, I still conclude the fragments are indeed the momentum matched fragments of Reaction R1. It can be seen in Figure 5.13 that the fits actually are identical for high translational energy releases, or stated another way, for the $\mathrm{N}_{3}$ product fragments which have the lowest amount of internal energy. The explanation for the observed difference is as follows: $\mathrm{CH}_{3}$ is a much more stable fragment with respect to unimolecular dissociation than $\mathrm{N}_{3}$, and under these energetic conditions, I would not expect it to have significant dissociation. $\mathrm{N}_{3}$, 

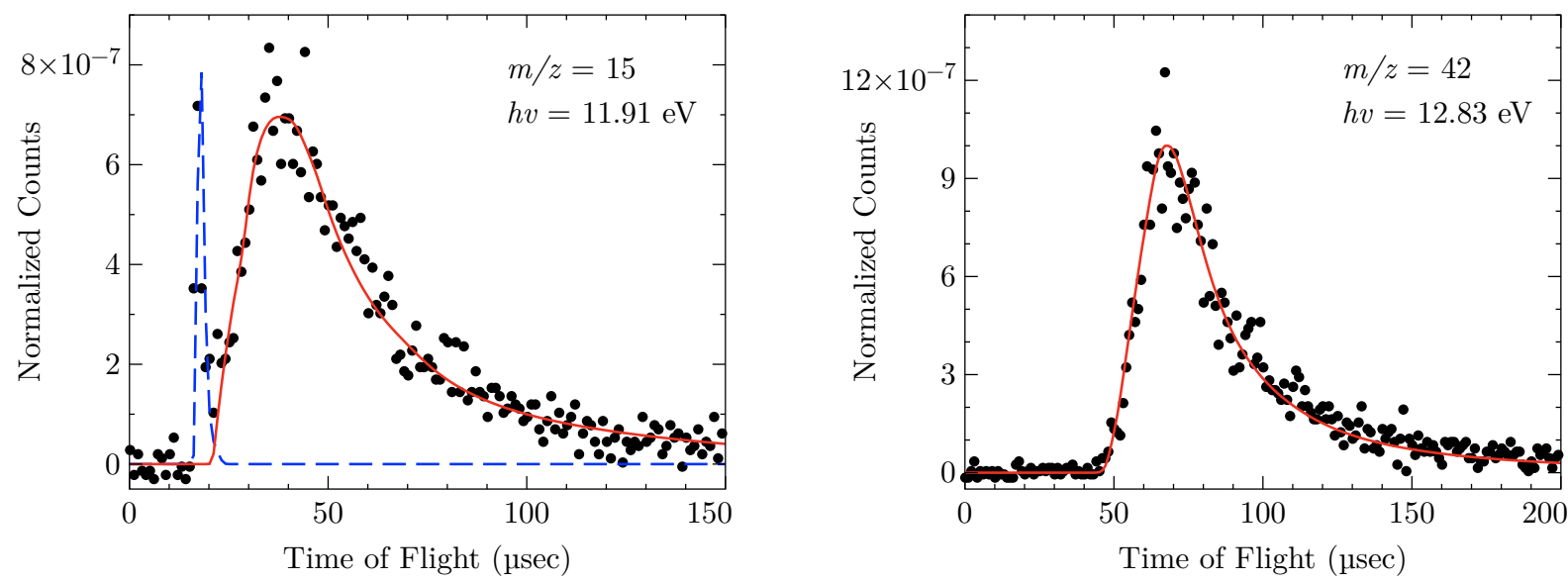

Figure 5.12: Time of flight spectra for $\mathrm{m} / z=15$ and 42 in the $193 \mathrm{~nm}$ photolysis of methyl azide. The solid black points are the experimental data and the red line is the result of the forward convolution fit for the translational energy distributions in Figure 5.13. The dashed blue line in the left panel is the previously discussed contribution from $\mathrm{CH}_{3}$ I. Spectra have been normalized for laser power, number of laser shots, and synchrotron radiation intensity. The laboratory angle was $30^{\circ}$ and the photoionization energy was $11.91 \mathrm{eV}$ for $m / z=15$ and $12.83 \mathrm{eV}$ for $m / z=42$. Left: $m / z=15$. Right: $m / z=42$.

however, from Figure 5.11 is has a barrier to dissociating to $\mathrm{N}_{2}+\mathrm{N}\left({ }^{2} \mathrm{D}\right)$ which is within reach for low translational energy releases. The difference between the two $P\left(E_{T}\right)$ distributions represents the fraction of $\mathrm{N}_{3}$ molecules with high enough internal energy to undergo unimolecular dissociation. The observation of this dissociation channel is significant, as it is the first experimental observation of the $\mathrm{CH}_{3}+\mathrm{N}_{3}$ radical dissociation channel in the photochemical decomposition of methyl azide.

\section{Molecular Elimination Channel}

I also observed the $\mathrm{N}_{2}$ molecular elimination channel at $193 \mathrm{~nm}$, as also seen in $248 \mathrm{~nm}$ photolysis. The data obtained at $193 \mathrm{~nm}$ is more complicated to analyze, and the interpretation is less definitive due to some unfortunate gaps in the data obtained at this wavelength. A representative TOF spectrum for $m / z=29$ is shown in Figure 5.14 along with the $P\left(E_{T}\right)$ used to fit this data.

The photoionization energy used throughout the entire $193 \mathrm{~nm}$ experiment is too low to ionize molecular nitrogen, the expected momentum matched product to the $\mathrm{m} / z=29$ fragment. Unfortunately, this means no data was taken at a high enough photoionization energy to observe the $\mathrm{N}_{2}$ fragment. The lack of direct observation of this partner fragment makes the interpretation of these results less definitive. The fit to $m / z=29$ is shown as a solid red line in Figure 5.14 and was created by the forward convolution fitting to this single fragment.

A product fragment was observed at $m / z=28$ despite the low ionization energy. I 


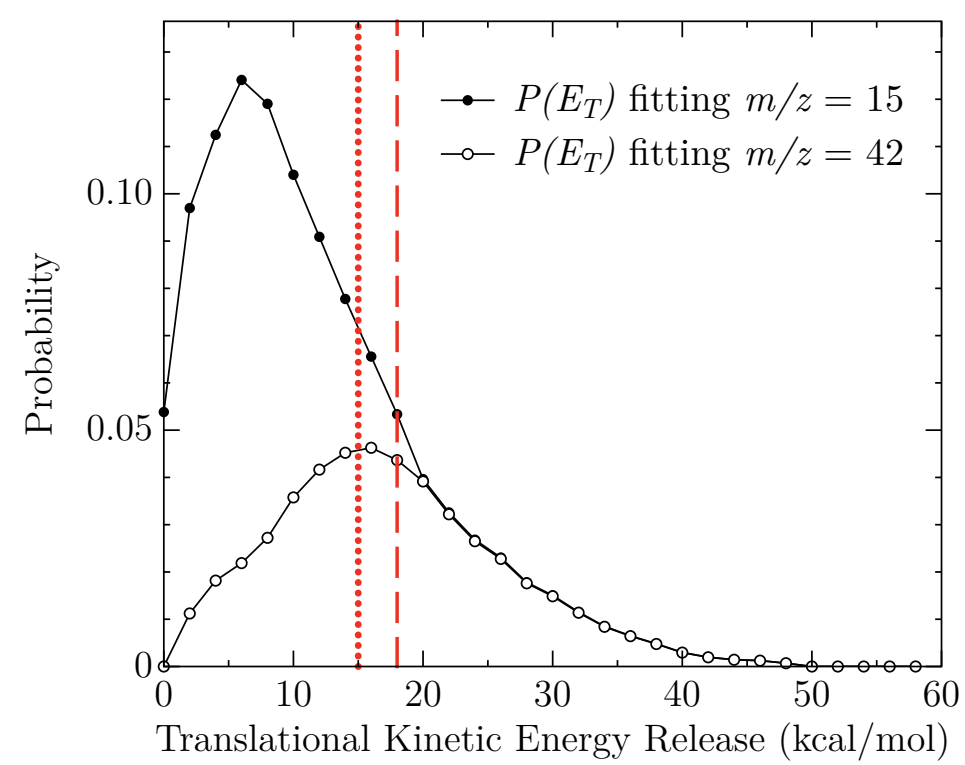

Figure 5.13: Center of mass translational energy release probability distribution, $P\left(E_{T}\right)$, used to fit $m / z=15$ and 42 fragments from $193 \mathrm{~nm}$ photolysis. The solid circles fit the $m / z=15$ fragment data and the open circles fit the $m / z=42$ fragment data. The dashed red line represents the energy barrier to linear $\mathrm{N}_{3}$ unimolecular dissociation and the dotted red line represents the barrier for cyclic $\mathrm{N}_{3}$ unimolecular dissociation.

assign this to the secondary dissociation channel $\mathrm{CH}_{2} \mathrm{~N}$ fragments produced by the first $\mathrm{H}$ atom loss step from Reaction S3, analogous to the analysis of the low ionization energy $m / z=28$ peak observed in the $248 \mathrm{~nm}$ data.

\subsubsection{Assignment of Secondary Dissociation Channels}

\section{Decomposition of Azide Fragment}

My interpretation of the difference in the $m / z=15$ and $42 P\left(E_{T}\right)$ diagrams requires that $\mathrm{N}_{3}$ undergo unimolecular dissociation to $\mathrm{N}_{2}+\mathrm{N}$. As mentioned in the analysis of the $\mathrm{N}_{2}$ elimination channel, no data could be obtained at a high enough photoionization energy to ionize molecular $\mathrm{N}_{2}$, so I unfortunately do not observe one of the crucial secondary dissociation products to support this theory. However, data was obtained for the momentum matched secondary dissociation fragment, $m / z=14$. The time of flight data, its forward convolution generated fit, and the secondary $P\left(E_{T}\right)$ can be seen in Figure 5.15.

The photoionization energy was tuned to $13.31 \mathrm{eV}$ in acquiring this data. This photon energy is not high enough to ionize the spin forbidden dissociation product ${ }^{N}\left({ }^{4} \mathrm{~S}\right)$ or dissociatively ionize $\mathrm{CH}_{3}$. The peak should belong only to the spin allowed dissociation product of $\mathrm{N}_{3}$ decomposition, $\mathrm{N}\left({ }^{2} \mathrm{D}\right)$.

The fit to this data was performed by secondary forward convolution fitting in ANALMAX. 

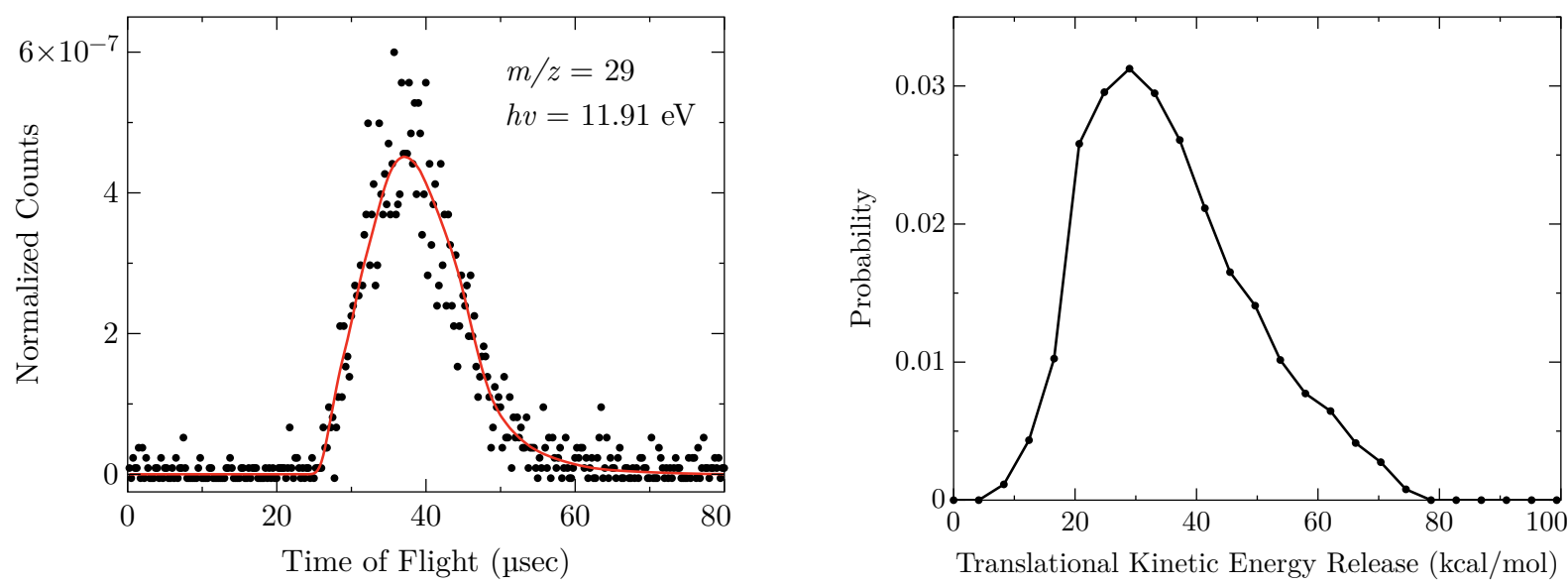

Figure 5.14: Time of flight spectrum for $m / z=29$ in $193 \mathrm{~nm}$ photolysis and the forward convolution fitting derived $P\left(E_{T}\right)$ which fits the observed data. Spectrum has been normalized for laser power, number of laser shots, and synchrotron radiation intensity. The laboratory angle is $30^{\circ}$ and the photoionization energy is $11.91 \mathrm{eV}$.

I have found a translational energy dependence on the secondary dissociation of $\mathrm{N}_{3}$. The difference between the $\mathrm{CH}_{3}$ and $\mathrm{N}_{3} P\left(E_{T}\right)$ was used as the primary $P\left(E_{T}\right)$ for fitting the secondary dissociation. This difference represents the translational energy distribution of the molecules which undergo dissociation. The resulting secondary translational energy distribution is given in Figure 5.15. I will return to a discussion of the translational energy result in the context of evidence for which geometry the $\mathrm{N}_{3}$ product has in Section 5.3.6.

\section{Decomposition of Methanimine Fragment}

As mentioned above, only low ionization energy $m / z=28$ spectra were observed in the $193 \mathrm{~nm}$ experiment. Since the ionization energy is much too low to be a primary dissociation product, I attribute this fragment to the secondary dissociation product $\mathrm{CH}_{2} \mathrm{~N}$. Representative spectra of the $\mathrm{m} / z=28$ and 27 fragments in the $193 \mathrm{~nm}$ dissociation process are shown in Figure 5.16.

The left panel shows the $m / z=28$ fragment TOF spectrum obtained at $11.91 \mathrm{eV}$ ionization energy - far below the ionization threshold of molecular nitrogen. The fitting was done as a secondary dissociation in ANALMAX. As discussed in the $248 \mathrm{~nm}$ data, the low ionization threshold $m / z=28$ fragment was determined to be either the intermediate in sequential $\mathrm{H}$ atom loss steps from methanimine which ultimately produced HCN, or a dissociative ionization event of the highly excited methanimine fragment. This analysis is reasonable here as I also observed the $\mathrm{HCN}$ fragment at $m / z=27$. I fit the peak with the same methodology used before - a secondary dissociation translational energy release peaking sharply near zero in ANALMAX which creates a good fit to the observed data.

The right panel of Figure 5.16 shows a $m / z=27$ TOF spectrum from $193 \mathrm{~nm}$ photolysis. 

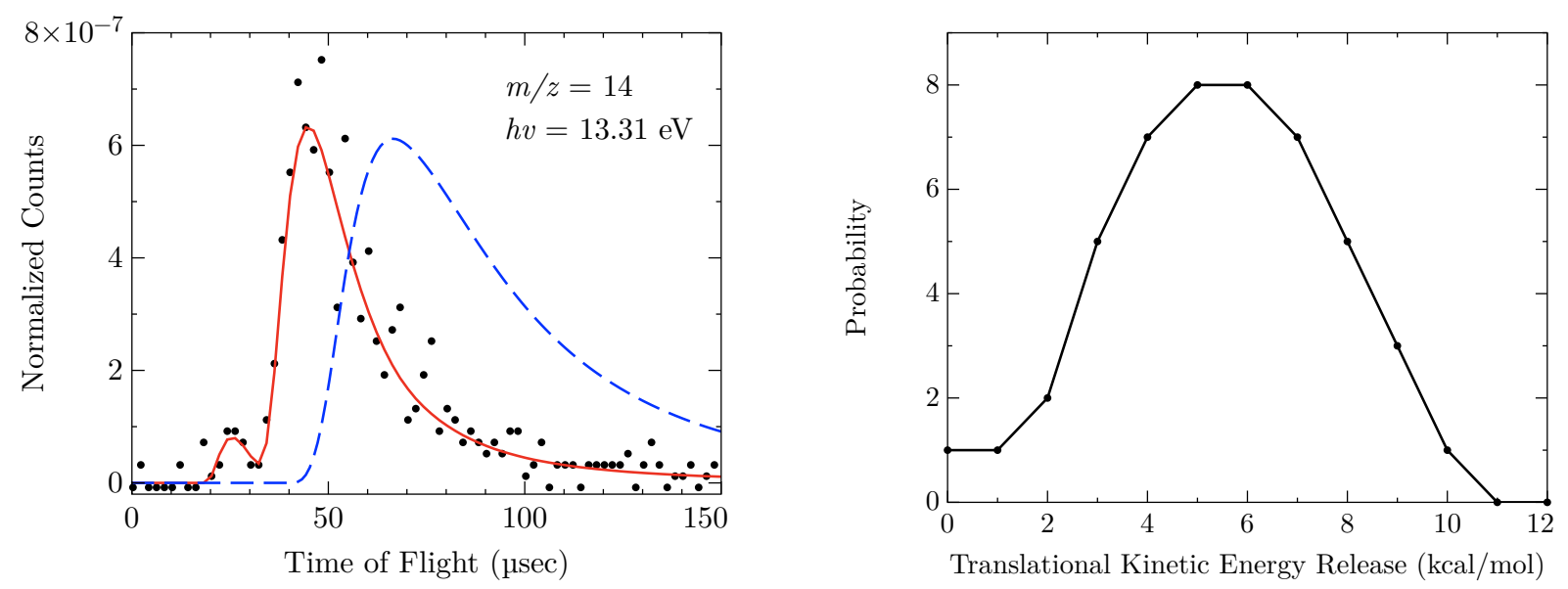

Figure 5.15: Time of flight spectra for $m / z=14$ in $193 \mathrm{~nm}$ photolysis and the forward convolution fitting derived secondary $P\left(E_{T}\right)$ which fits the observed data when the difference between $P\left(E_{T}\right)$ s in Figure 5.13 is used as the parent fragment primary $P\left(E_{T}\right)$. The dashed blue line shows the TOF predicted from a secondary $P\left(E_{T}\right)$ which would be expected for linear $\mathrm{N}_{3}$ dissociation. Spectrum has been normalized for laser power, number of laser shots, and synchrotron radiation intensity. The laboratory angle is $30^{\circ}$ and the photoionization energy is $13.31 \mathrm{eV}$.

In the $248 \mathrm{~nm}$ analysis, I concluded that there were in fact two mechanisms, one which produced $\mathrm{HNC}$ through a molecular $\mathrm{H}_{2}$ elimination and one producing $\mathrm{HCN}$ through sequential $\mathrm{H}$ atom loss steps. I have already shown the low ionization threshold $\mathrm{m} / z=28$ fragment at this wavelength which could be the intermediate step in the HCN formation mechanism. The shape of the peak for $\mathrm{m} / z$ does not seem to have a dependence on ionization energy as was seen at $248 \mathrm{~nm}$ and, in fact, can be seen at $11.91 \mathrm{eV}$ - below the threshold for both HCN and HNC. The fit to the spectrum was created with a secondary translational energy release which peaked near zero, implying that the signal is due to HCN formed in sequential $\mathrm{H}$-atom loss steps, rather than $\mathrm{H}_{2}$ molecular elimination which would be expected to show a more substantial energy release. If this is the case, then it must be in a highly excited state to be detectable at such low photoionization energies. This is another characteristic of the $\mathrm{N}_{2}$ molecular elimination reaction which I cannot definitively explain with the current data. In summary: a $m / z=27$ product is observed, which can only come from secondary dissociation of the molecular elimination channel. Whether the peak can be assigned to only HCN or some combination of HCN and HNC is unclear, but the evidence suggests $\mathrm{HCN}$ is the dominant pathway at this wavelength.

\subsubsection{Photoionization Threshold}

One additional set of data was obtained at $193 \mathrm{~nm}$ to determine the dependance of the $m / z=42$ signal on the photoionization energy of the synchrotron radiation. This results 

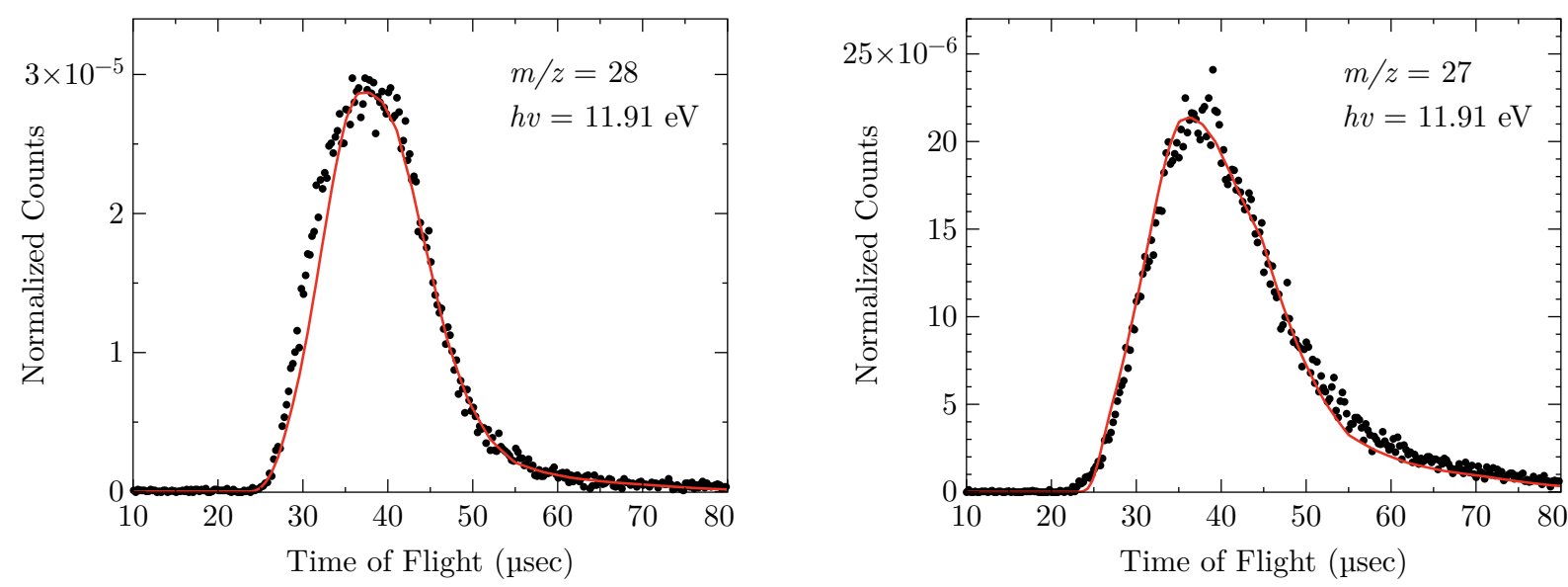

Figure 5.16: Time of flight spectra for $\mathrm{m} / z=28$ and 27 in the $193 \mathrm{~nm}$ photolysis of methyl azide. The solid black points are the experimental data and the red line is the result of secondary forward convolution fitting from the $P\left(E_{T}\right)$ in Figure 5.14 with a near zero translational energy release for the secondary dissociation. Spectra have been normalized for laser power, number of laser shots, and synchrotron radiation intensity. The laboratory angle was $30^{\circ}$ and the photoionization energy was $11.91 \mathrm{eV}$ for both spectra. Left: $m / z=28$. Right: $m / z=27$.

in a measurement of the ionization threshold of the $m / z=42$ photofragment. A similar measurement was carried out in the $\mathrm{ClN}_{3}$ work of Samartzis et al.[56]. Figure 5.17 shows the integrated TOF signal plotted against the photoionization energy of the synchrotron radiation.

Each point in the figure represents the integrated number of counts in an individual $\mathrm{N}_{3}$ TOF spectrum. Care was taken to normalize the integrated intensity to the variations in laser power, number of laser shots, and synchrotron radiation intensity. The intersection of the two linear fits to these points provides a reasonable estimation of the ionization threshold of the $\mathrm{N}_{3}$ product fragment produced in the $193 \mathrm{~nm}$ photolysis. The ionization threshold was determined to be $10.7 \mathrm{eV}$ with this method. The dashed red line in the figure shows the known ionization potential of linear $\mathrm{N}_{3}$, while the dotted line shows the ionization potential of cyclic $\mathrm{N}_{3}$. The shaded area represents the bandwidth of the energy of the ionizing synchrotron radiation. This threshold is consistent with the production on cyclic $\mathrm{N}_{3}$, with no observed second threshold at the linear isomer ionization potential.

\subsubsection{Evidence for Linear vs. Cyclic States of the Azide Radical}

From the energy diagram in Figure 5.11, evidence can be found for whether linear or cyclic $\mathrm{N}_{3}$ (or both) is produced in this photolysis process. Both linear and cyclic forms were observed in the photodissociation of $\mathrm{ClN}_{3}$ at $248 \mathrm{~nm}$ [56]. The TOF data from that experiment clearly shows a bimodal distribution for the $\mathrm{CH}_{3}$ and $\mathrm{N}_{3}$ fragments. The 


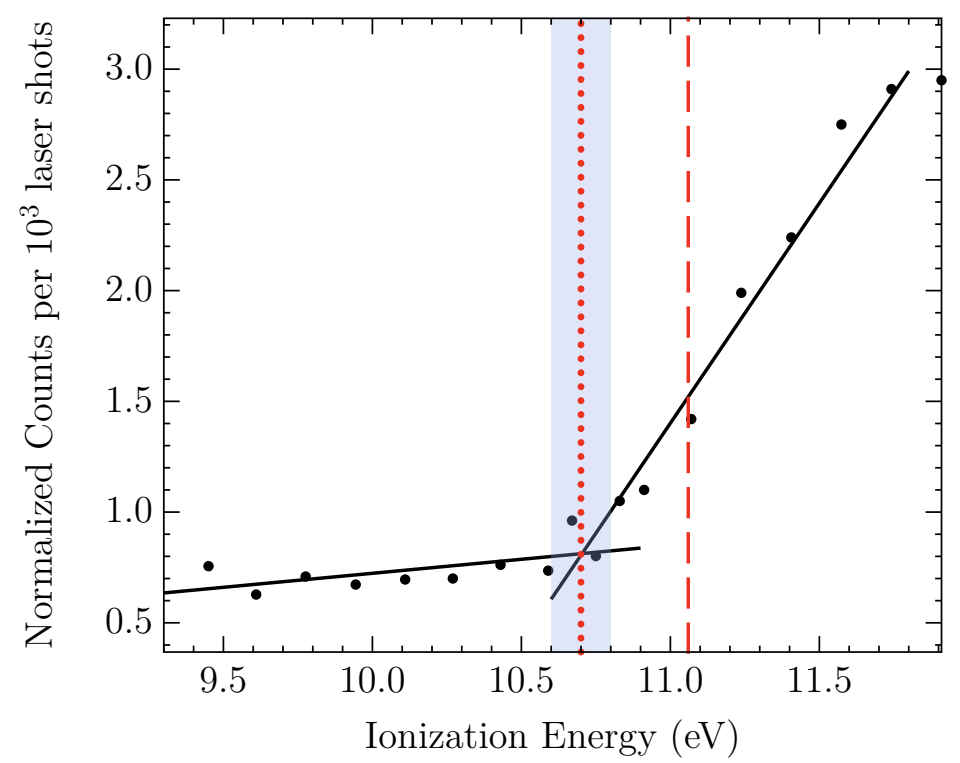

Figure 5.17: Photoionization threshold of $m / z=42$. The black points are the integrated intensity of the same region of the TOF spectrum for $m / z=42$ at different photoionization energies. The dotted red line shows the expected ionization potential for cyclic $\mathrm{N}_{3}$ and the dashed red line is the expected ionization threshold for linear $\mathrm{N}_{3}$. The shaded region represents the bandwidth of the synchrotron radiation.

current experiment's data does not show any evidence of a bimodal distribution in either the $m / z=15$ or 42 TOF spectra, making it unlikely that both linear and cyclic forms are present. The center of mass translational energy release determined for this channel has a maximum energy of $45 \pm 4 \mathrm{kcal} / \mathrm{mol}$. From Figure 5.11, this is extremely close to the limit predicted by the heats of formation for a cyclic $\mathrm{N}_{3}$ channel $(48$ or $50 \mathrm{kcal} / \mathrm{mol}$ depending on which heat of formation one uses for cyclic $\mathrm{N}_{3}$ ). Linear $\mathrm{N}_{3}$ would have an $E_{\max }$ of $78 \mathrm{kcal} / \mathrm{mol}$. That would leave nearly $33 \mathrm{kcal} / \mathrm{mol}$ of energy unaccounted for. The excellent agreement of the maximum energy release to the previously observed energy of the cyclic $\mathrm{N}_{3}$ channel is the first piece of evidence for cyclic $\mathrm{N}_{3}$ in this experiment.

Next, I return to the $m / z=14$ data, where I determined a maximum secondary translational energy of $11 \mathrm{kcal} / \mathrm{mol}$, with a peak in the distribution at $6 \mathrm{kcal} / \mathrm{mol}$ (see Figure 5.15). Looking to the energy diagram, one can see this maximum energy is in good agreement with the barrier height for the spin allowed dissociation of cyclic $\mathrm{N}_{3}$. The deviation of the $\mathrm{N}_{3} P\left(E_{T}\right)$ from the $\mathrm{CH}_{3} P\left(E_{T}\right)$ begins for molecules with about $18 \mathrm{kcal} / \mathrm{mol}$ of translational energy. This is in closer agreement to the energy barrier for dissociation of the linear state.

In addition to the available energy, there is also a larger exit barrier in the dissociation from cyclic $\mathrm{N}_{3}$ than from linear $\mathrm{N}_{3}(7 \mathrm{kcal} / \mathrm{mol}$ vs. $3 \mathrm{kcal} / \mathrm{mol})$. Figure 5.15 has a second fit shown in it represented by a dashed blue line. This is a simulated secondary dissociation 
event which peaks at around $2 \mathrm{kcal} / \mathrm{mol}$ - more inline with the expected energy release for the $3 \mathrm{kcal} / \mathrm{mol}$ exit barrier for linear $\mathrm{N}_{3}$ dissociation. This fit is clearly inconsistent with the observed data. This provides a further piece of evidence which leans more toward the production of cyclic $\mathrm{N}_{3}$ over linear.

The results of the photoionization threshold provide the final evidence. I find the ionization threshold of the $m / z=42$ fragment to be $10.7 \mathrm{eV}$ - in good agreement with both the theoretically predicted ionization threshold for neutral cyclic $\mathrm{N}_{3}$ to the cationic state (10.595 eV [3]) and the experimentally determined threshold in the previous $\mathrm{ClN}_{3}$ work (10.6 eV [56]). There is no obvious second threshold around the known ionization potential of linear $\mathrm{N}_{3}(11.06 \mathrm{eV}$ [22]).

Taken individually, none of these results is undeniable proof of cyclic $\mathrm{N}_{3}$ formation. However, taken all together, the results are more consistent with the expectations for the cyclic form of $\mathrm{N}_{3}$. The end result is that not only is the radical dissociation channel possible in the photolysis of $\mathrm{CH}_{3} \mathrm{~N}_{3}$ at $193 \mathrm{~nm}$, it may be producing the cyclic $\mathrm{N}_{3}$ radical exclusively.

\subsubsection{Comment on Branching Ratio of Primary Dissociation Channels}

A comment on the branching ratio between the observed primary dissociation channels I have clear data for two reaction channels in the $193 \mathrm{~nm}$ dissociation, so one may think I could determine a reasonable estimate of the branching ratio between the two channels. This is, unfortunately, not as straightforward as it appears. The main problem is the lack of known photoionization cross-sections for all of the observed molecules. Given the highly excited nature of some fragments and the lack of previously measured ionization cross-sections, I cannot report a numerical branching ratio. The best that can be said from the observations of this experiment is that fragments originating from both channels can be easily observed, so neither channel seems overwhelmingly dominant. A more accurate, quantitative assessment of the branching ratio could be obtained in an experiment similar to this one, using electron impact ionization. The electron impact ionization cross-sections can be reasonably well estimated theoretically and would provide a more quantitative result than this experiment is able to. 


\section{Chapter 6}

\section{Summary and Outlook}

Here I summarize the key results of both experiments, point out the differences observed between $248 \mathrm{~nm}$ and $193 \mathrm{~nm}$ photodissociation, and highlight the most important conclusions and impact of this work. Finally, I will present an overview of some results which were published by other researchers on this topic following the work done here and how those results complement my own.

\subsection{Summary}

Photofragment translational spectroscopy was performed on the molecule methyl azide at $248 \mathrm{~nm}$ and $193 \mathrm{~nm}$ dissociation wavelengths. Time of flight spectra were recorded for all resulting photofragments using tunable synchrotron radiation to ionize the fragments. This allows for both translational energy distributions and ionization energies to be used in the identification and assignment of the fragments. The following conclusions were made following the analysis of the experimental data:

\section{8 nm Photolysis}

- Identified the molecular $\mathrm{N}_{2}$ elimination channel as the only primary dissociation process in methyl azide at this wavelength.

- No observation of the radical dissociation channel producing $\mathrm{N}_{3}$.

- The translational energy distribution of products supports a mechanism with singlet methylnitrene, $\mathrm{CH}_{3} \mathrm{~N}$, as the initial product.

- Significant secondary dissociation of the $\mathrm{m} / z=29$ product fragment is observed, supporting the isomerization of methylnitrene to a highly internally excited state of methanimine.

- Two channels identified in the secondary dissociation products of methanimine - one producing hydrogen cyanide, HCN, and the other producing hydrogen isocyanide, HNC.

- No evidence observed for reactions taking place on the triplet potential energy surface. 


\section{3 nm Photolysis}

- Identified both the molecular $\mathrm{N}_{2}$ elimination and radical dissociation channels as primary photolysis pathways for methyl azide at this wavelength.

- The branching ratio between these channels could not be determined from the available data and is a significant question.

- $\mathrm{N}_{3}$ product fragments with high internal energy were observed to undergo significant unimolecular dissociation to $\mathrm{N}+\mathrm{N}_{2}$. The nitrogen atom from secondary dissociation was identified in the $m / z=14$ fragment data.

- The molecular $\mathrm{N}_{2}$ elimination channel is likely similar to the $248 \mathrm{~nm}$ dissociation from the available information, but the absence of data on the $\mathrm{N}_{2}$ product fragment at this wavelength makes a complete assignment unavailable.

- At this wavelength, it appear that the secondary dissociation from the $m / z=29$ fragment only produces one product which is more consistent with HCN.

- Measured the photoionization threshold of the $\mathrm{N}_{3}$ product fragment. The ionization energy is consistent with the available data for the cyclic $\mathrm{N}_{3}$ isomer.

- All experimental evidence supports cyclic $\mathrm{N}_{3}$ as being the only conformation produced in the photodissociation of methyl azide at this wavelength.

- Despite the unimolecular decomposition, a large fraction of the cyclic $\mathrm{N}_{3}$ survives on the 50 to 100 usec time scale to the detector.

The photodissociation dynamics of methyl azide show two notable differences in the results between the two dissociation wavelengths.

At $248 \mathrm{~nm}$, only fragments related to the molecular $\mathrm{N}_{2}$ elimination channel was observed. No radical channel fragments were observed in the $248 \mathrm{~nm}$ photolysis. At $193 \mathrm{~nm}$, the fragments which originate in the radical channel are observed in addition to the fragments from the molecular elimination channel. This suggests the appearance threshold for the radical channel is somewhere between $248 \mathrm{~nm}$ and $193 \mathrm{~nm}$.

There is also a difference in the observed behavior in the secondary dissociation products. The secondary dissociation of methanimine at $248 \mathrm{~nm}$ appears to have two different mechanisms and produces fragments consistent with both hydrogen cyanide and hydrogen isocyanide. At $193 \mathrm{~nm}$, only one product is observed with little translational energy release, more consistent with the mechanism producing hydrogen cyanide. The reason the isocyanide is not observed is uncertain. It could be both are still present, but the fragments just cannot be distinguished from one another at this wavelength.

This is the first photolysis study of methyl azide under collision free conditions at any wavelength. The $248 \mathrm{~nm}$ photolysis results provide new information regarding the debate over singlet methylnitrene existing as a discrete intermediate or concerted methanimine production in the dissociation of methyl azide. While still not able to directly trap or 
observe the singlet methylnitrene, the translational energy release following the dissociation is consistent with some of the energy being tied up in a transition state structure. The $193 \mathrm{~nm}$ photolysis provides the first experimental observation of the radical channel occurring in methyl azide decomposition. Furthermore, the properties of the $\mathrm{N}_{3}$ fragment are consistent only with the cyclic form, which was not even known to exist until recently. Even more important, a higher fraction of this cyclic $\mathrm{N}_{3}$ survives on the time scale to the detector than in any previous experiments observing this cyclic structure, making it a good precursor for further study of the properties of this illusive molecule.

\subsection{Further Work}

A direct follow-up experiment was conducted by Alfredo Quinto-Hernandez and the results published in 2012 [48]. In this experiment, $193 \mathrm{~nm}$ photolysis of methyl azide was again investigated, however, electron impact (EI) ionization was used. The EI ionization cross sections are able to be predicted with good accuracy, allowing this experiment to determine the branching ratio between the primary dissociation channels.

The results of this experiment confirmed my observation of both the molecular $\mathrm{N}_{2}$ elimination channel and the radical $\mathrm{N}_{3}$ channels. The branching ratio reveals $98.3 \%$ of dissociation events belong to the molecular elimination channel. Only about $1.7 \%$ resulted in the radical channel. Compared to my own data, these results are consistent with my observations. Although fragments from both channels are easily observed in my experiment, comparing the normalized intensity of the $m / z=15$ fragment from the radical channel to the $m / z=27$ or 28 from the molecular elimination channel, the radial channel is on the order of $10^{-7}$ and the molecular elimination on the order of $10^{-5}$. This results in a similar $99 \%$ to $1 \%$ branching ratio if the ionization cross sections are considered to be equal.

The experiment was also able to identify the branching ratio between linear and cyclic $\mathrm{N}_{3}$ as being $95.3 \%$ in favor of the cyclic structure. I was unable to observe any linear $\mathrm{N}_{3}$ in my experiment, and this may be why. It appears to be just $5 \%$ of a channel which is only $1 \%$ of the dissociation events. This also confirms my analysis of cyclic $\mathrm{N}_{3}$ production in the photolysis of methyl azide.

A theoretical IR absorption spectra for the cyclic structure of $\mathrm{N}_{3}$ was published by Babikov et al. in 2010 [2]. Modeling the IR absorption of this molecule is complicated due to the unusual effects which arise from the molecule's conical intersection. This is an important theoretical prediction. With experimental evidence of how to produce the cyclic isomer and a prediction of its spectroscopic nature, it may soon be possible to perform a direct IR spectroscopy study on this molecule. Results from that experiment could provide undeniable evidence for the existence of the cyclic structure. 


\subsection{Experimental Outlook}

As a result of the experimental and theoretical studies of methyl azide photolysis, a few interesting questions remain to be answered and new experiments can be proposed.

Methyl azide and chlorine azide both appear to be favorable to production of cyclic $\mathrm{N}_{3}$ at higher ionization energies. Methyl azide at $193 \mathrm{~nm}$ is $95 \%$ in favor of the cyclic structure and chlorine azide appears to be exclusive to the cyclic form at $157 \mathrm{~nm}$. The cyclic molecule produced in the methyl azide photolysis appears to be more stable toward secondary dissociation as desired. However, the branching ratio between molecular and radical channels invert between the methyl and chlorine azides. Chlorine azide was shown to be about $95 \%$ in favor of the radical channel while methyl azide appears to be almost $99 \%$ in favor of the molecular nitrogen elimination channel. It could be the stability toward the isomerization of the $\mathrm{CH}_{2} \mathrm{NH}$ molecule which leads to this change in behavior. Replacing the hydrogen in the methyl group with a larger halogen, like fluorine, may make a molecule like $\mathrm{CF}_{3} \mathrm{~N}_{3}$ an interesting next candidate for photolysis studies. This may maintain the preference toward stable cyclic $\mathrm{N}_{3}$ fragments and the high selectivity of both the cyclic structure and the radical dissociation channel.

With the new IR absorption structure prediction, it would be of great interest to try using chlorine or methyl azide as a precursor to producing cyclic $\mathrm{N}_{3}$ for spectroscopic identification. Conformation of the predicted spectra would be a huge step forward in proving that the high energy form of $\mathrm{N}_{3}$ observed in these experiments is really the cyclic structural form.

With more sources of cyclic $\mathrm{N}_{3}$ being identified, a cross molecular beam reaction between the cyclic fragments and a beam of $\mathrm{N}\left({ }^{2} \mathrm{D}\right)$ atoms may be feasible to test the prediction that this reaction will produce tetraazatetrahedrane. This would be a new homonuclear polynitrogen molecule and is predicted to be stable and useful for energy storage. 


\section{Bibliography}

[1] J.F. Arenas et al. "A multiconfigurational self-consistent field study of the thermal decomposition of methyl azide". In: J. Chem. Phys. 111.2 (1999), pp. 551-561.

[2] D. Babikov and B.K. Kendrick. "The infrared spectrum of cyclic- $\mathrm{N}_{3}$ : Theoretical prediction". In: J. Chem. Phys. 133.17 (2010), p. 174310.

[3] D. Babikov, V.A. Mozhayskiy, and A.I. Krylov. "Conical and glancing Jahn-Teller intersections in the cyclic trinitrogen cation". In: J. Chem. Phys. 124 (2006), p. 224309.

[4] M.D. Barry and P.A. Gorry. "Photofragmentation dynamics of $\mathrm{CH}_{3} \mathrm{I}$ at $248 \mathrm{~nm}$ ". In: Mol. Phys. 52.2 (1984), pp. 461-473.

[5] G. Bieri and B.O. Jonsson. "HNC ${ }^{+}$radical cation studied by charge-exchange mass spectrometry". In: Chem. Phys. Lett. 56.3 (1978), pp. 446-449.

[6] M. Bittererova, H. Ostmark, and T. Brinck. "A theoretical study of the azide $\left(\mathrm{N}_{3}\right)$ doublet states. A new route to tetraazatetrahedrane $\left(\mathrm{N}_{4}\right): \mathrm{N}+\mathrm{N}_{3} \rightarrow \mathrm{N}_{4}$ ". In: $J$. Chem. Phys. 116.22 (2002), pp. 9740-9748.

[7] H. Bock and R. Dammel. "The Pyrolysis of Azides in the Gas Phase". In: Angew. Chem. Int. Ed. 26.6 (1987), pp. 504-526.

[8] T. Boublík and K. Aim. "Heats of vaporization of simple non-spherical molecule compounds". In: Collect. Czech. Chem. Commun. 37 (1972), p. 3513.

[9] J. Buckingham and S.M. Donaghy. Dictionary of Organic Compounds. 5th. Chapman and Hall, 1982.

[10] G.E. Busch et al. "Photofragment Spectrometer". In: Rev. Sci. Instrum. 41.7 (1970), p. 1066.

[11] W.A. Chupka and C. Lifshitz. "Photoionization of $\mathrm{CH}_{3}$; Heat of Formation of $\mathrm{CH}_{2}$ *". In: J. Chem. Phys. 48.3 (1968), p. 1109.

[12] R.E. Continetti, B.A. Balko, and Y.T. Lee. "Symmetric stretch excitation of $\mathrm{CH}_{3}$ in the $193.3 \mathrm{~nm}$ photolysis of $\mathrm{CH}_{3} \mathrm{I}$ ". In: J. Chem. Phys. 89.5 (1988), pp. 3383-3384.

[13] R.E. Continetti et al. "Fast beam studies of $\mathrm{N}_{3}$ photodissociation". In: Chem. Phys. Lett. 182.5 (1991), pp. 406-411.

[14] R.E. Continetti et al. "Photodissociation dynamics of the $\mathrm{N}_{3}$ radical". In: J. Chem. Phys. 99.4 (1993), pp. 2616-2631.

[15] P.A. Cook, S.R. Langford, and M.N.R. Ashfold. "The ultraviolet photodissociation of $\mathrm{HN}_{3}$ : the $\mathrm{H}+\mathrm{N}_{3}$ product channel". In: Phys. Chem. Chem. Phys. 1 (1999), pp. 4555. 
Bibliography

[16] C.L. Currie and B.D. Darwent. "The photochemical decomposition of methyl azide". In: Can. J. Chem. 41.6 (1963), p. 1552.

[17] T. Curtius. "Ueber Stickstoffwasserstoffsäure (Azoimid) $\mathrm{N}_{3} \mathrm{H}$ ". In: Chem. Ber. 23 (1890), pp. 3023-3033.

[18] N.R. Daly. "Scintillation Type Mass Spectrometer Ion Detector". In: Rev. Sci. Instrum. 31.3 (1960), pp. 264-267.

[19] O. Dimroth and W. Wislicenus. "Ueber das Methylazid". In: Chem. Ber. 38 (1905), pp. $1573-1576$.

[20] A.E. Douglas and W.J. Jones. "The $2700 \AA$ bands of the $\mathrm{N}_{3}$ molecule". In: Can. J. Chem. 43 (1965), p. 2216.

[21] V. Dribinski et al. "Reconstruction of Abel-transformable images: The Gaussian basis-set expansion Abel transform method". In: Rev. Sci. Instrum. 73 (2002), pp. 2634 2642.

[22] J.M. Dyke et al. "Vacuum ultraviolet photoelectron spectroscopy of transient species". In: Mol. Phys. 47.5 (1982), pp. 1231-1240.

[23] F.R. Elder, R.V. Langmuir, and H.C. Pollock. "Radiation from Electrons Accelerated in a Synchrotron". In: Phys. Rev. 74.1 (1948), p. 52.

[24] U. Even et al. "Cooling of large molecules below $1 \mathrm{~K}$ and He clusters formation". In: J. Chem. Phys. 112.18 (2000), pp. 8068-8071.

[25] C. Fridh and L. Asbrink. "Photoelectron and electron impact spectrum of HCN". In: J. Electron. Spectrosc. Relat. Phenom. 7.2 (1975), pp. 119-138.

[26] N. Getoff, R. Laupert, and R.N. Schindle. "Photolysis of methylazide in gaseous phase". In: Z. Phys. Chem. Neue Folge 70.1-2 (1970), p. 70.

[27] P. Griess. "On a New Class of Compounds in which Nitrogen is substituted for Hydrogen". In: Proc. R. Soc. London 13 (1863-1864), pp. 375-384.

[28] N. Hansen and A.M. Wodtke. "Velocity Map Ion Imaging of Chlorine Azide Photolysis: Evidence for Photolytic Production of Cyclic-N, ${ }_{3}$ ". In: J. Phys. Chem. A 107.49 (2003), pp. 10608-10614.

[29] N. Hansen et al. "Ion dissociation dynamics of the chlorine azide cation $\left(\mathrm{ClN}_{3}^{+}\right)$ investigated by velocity map imaging". In: J. Chem. Phys. 118.23 (2003), pp. 1048510493.

[30] N. Hansen et al. "Photodissociation dynamics of $\mathrm{ClN}_{3}$ at $203 \mathrm{~nm}$ : the $\mathrm{NCl}\left(\mathrm{a}^{1} \Delta / \mathrm{X}^{3} \Sigma^{-}\right)$ product branching ratio". In: Chem. Phys. Lett. 368.5-6 (2003), pp. 568-573.

[31] N. Hansen et al. "Photofragment translation spectroscopy of $\mathrm{ClN}_{3}$ at $248 \mathrm{~nm}$ : Determination of the primary and secondary dissociation pathways". In: J. Chem. Phys. 123.10 (2005), p. 104305.

[32] S.A. Harich. PHOTRAN: A program for forward convolution analysis of photodissociation. 2003. 
[33] A. Hassner et al. "Utility of a Polymeric Azide Reagent in the Formation of Di- and Triazidomethane. Their NMR-Spectra and the X-ray Structure of Derived Triazoles". In: J. Org. Chem. 55.8 (1990), pp. 2304-2306.

[34] E.J. Hintsa, A.M. Wodtke, and Y.T. Lee. "Infrared Multiphoton Dissociation of Ethyl and Methyl Acetate". In: J. Phys. Chem. 92.19 (1988), pp. 5379-5387.

[35] K.P. Huber and G. Herzberg. Molecular Spectra and Molecular Structure. IV. Constants of diatomic molecules. Van Nostrand Reinhold Co., 1979.

[36] D. Ivanenko and I. Pomeranchuk. "On the Maximal Energy Attainable in a Betatron". In: Phys. Rev. 65 (1944), p. 343.

[37] A.V. Komissarov et al. "Rate Constants for Quenching and Self-Annihilation of NCl(a $\left.{ }^{1} \Delta\right)$ ". In: J. Phys. Chem. A 106 (2002), pp. 8427-8434.

[38] C. Larson et al. "Collision-free photochemistry of methylazide: Observation of unimolecular decomposition of singlet methylnitrene". In: J Chem Phys 125.13 (2006), p. 133302 .

[39] C. Larson et al. "Observation of photochemical $\mathrm{C}-\mathrm{N}$ bond cleavage in $\mathrm{CH}_{3} \mathrm{~N}_{3}$ : A new photochemical route to cyclic $\mathrm{N}_{3}$ ". In: J. Phys. Chem. A 112.6 (2008), pp. 1105-1111.

[40] Y.T. Lee et al. "Molecular Beam Reactive Scattering Apparatus with Electron Bombardment Detector". In: Rev. Sci. Instrum. 40 (1969), p. 1402.

[41] J.A. Leermakers. "The Thermal Decomposition of Methyl Azide. A Homogeneous Unimolecular Reaction". In: J. Am. Chem. Soc. 55.8 (1933), pp. 3098-3105.

[42] A. Lienard. In: L'Eclairage elec. 16.5 (1898).

[43] Y. Liming et al. "Photodissociation of $\mathrm{CH}_{3} \mathrm{~N}_{3}$-Spectral evidences for the formation of triplet $\mathrm{CH}_{3} \mathrm{~N}$ radical". In: ACTA Physico-chimica Sinica 11.11 (1995), pp. 961-964.

[44] J.J. Lin et al. "Photodissociation dynamics of $\mathrm{CH}_{3} \mathrm{Cl}$ at $157.6 \mathrm{~nm}$ : Evidence for $\mathrm{CH}_{2}\left(\tilde{\mathrm{X}}^{3} \mathrm{~B}_{1} / \tilde{\mathrm{a}}^{1} \mathrm{~A}_{1}\right)+\mathrm{HCl}$ product channels". In: Chem. Phys. Lett. 361.5-6 (2002), pp. $374-382$.

[45] C.G. Morgan, M. Drabbels, and A.M. Wodtke. "State-specific neutral time-of-flight of CO from ketene photodissociation at $351 \mathrm{~nm}$ : The internal energy distribution of $\mathrm{CH}_{2}\left(\tilde{\mathrm{X}}^{3} \mathrm{~B}_{1}\right)$ ". In: J. Chem. Phys. 105.11 (1996), pp. 4550-4555.

[46] F.L. Nesbitt et al. "Measurement of the Photoionization Spectra and Ionization Thresholds of the $\mathrm{H}_{2} \mathrm{CN}$ and $\mathrm{D}_{2}$ CN Radicals". In: J. Phys. Chem. 95 (1991), pp. 76137617.

[47] M.T. Nguyen, D. Sengupta, and T.K. Ha. "Another Look at the Decomposition of Methyl Azide and Methanimine: How is HCN Formed?" In: J. Phys. Chem. 100.16 (1996), pp. 6499-6503.

[48] A. Quinto-Hernandez et al. "Photofragmentation Translational Spectroscopy of Methyl Azide (CH3N3) Photolysis at 193 nm: Molecular and Radical Channel Product Branching Ratio". In: J. Phys. Chem. A 116.19 (2012), 4695-4704. 
Bibliography

[49] A. Quinto-Hernandez et al. "Photoionization of $\mathrm{CH}_{3} \mathrm{~N}_{3}$ Produces ${ }^{3} \mathrm{~B}_{2} \mathrm{~N}_{3}^{-}$: A Theoretical and Experimental Study of the Ion-Pair Channel". In: J. Phys. Chem. Lett. 2.18 (2011), pp. 2311-2315.

[50] S.J. Riley and K.R. Wilson. "Excited Fragments from Excited Molecules: Energy Partitioning in the Photodissociation of Alkyl Iodides". In: Faraday Discuss. 53 (1972), pp. 132-146.

[51] B. Ruscic et al. "IUPAC Critical Evaluation of Thermochemical Properties of Selected Radicals. Part I". In: J. Phys. Chem. Ref. Data 34.2 (2005), pp. 573-656.

[52] P.C. Samartzis, N. Hansen, and A.M. Wodtke. "Imaging $\mathrm{ClN}_{3}$ photodissociation from 234 to 280 nm". In: Phys. Chem. Chem. Phys. 8.25 (2006), pp. 2958-2963.

[53] P.C. Samartzis and A.M. Wodtke. "All-nitrogen chemistry: how far are we from $\mathrm{N}_{60}$ ?" In: Int. Rev. Phys. Chem. 25.4 (2006), pp. 527-552.

[54] P.C. Samartzis and A.M. Wodtke. "Casting a new light on azide photochemistry: photolytic production of cyclic-N ${ }_{3}$ ". In: Phys. Chem. Chem. Phys. 9.24 (2007), pp. 30543066.

[55] P.C. Samartzis et al. "The simplest all-nitrogen ring: Photolytically filling the cyclic$\mathrm{N}_{3}$ well". In: J. Chem. Phys. 126.4 (2007), p. 041101.

[56] P.C. Samartzis et al. "Two photoionization thresholds of $\mathrm{N}_{3}$ produced by $\mathrm{ClN}_{3}$ photodissociation at $248 \mathrm{~nm}$ : Further evidence for cyclic $\mathrm{N}_{3}$ ". In: J. Chem. Phys. 123.5 (2005), p. 051101.

[57] H.R. Shang et al. "Dissociation dynamics of methylazide on the first excited singlet surface". In: Chem. Phys. Lett. 236.3 (1995), pp. 318-323.

[58] M.J. Travers et al. "Photoelectron spectroscopy of the $\mathrm{CH}_{3} \mathrm{~N}^{-}$ion". In: J. Chem. Phys. 111.12 (1999), pp. 5349-5360.

[59] P.G. Urben. Bretherick's Handbook of Reactive Chemical Hazards. 6th. Butterworth Heinemann Ltd, 1999.

[60] S.P. Walch. "Theoretical characterization of the potential energy surface for $\mathrm{H}+$ $\mathrm{N}_{2} \longrightarrow \mathrm{HN}_{2}$. II. Computed points to define a global potential". In: J. Chem. Phys. 93.4 (1990), pp. 2384-2392.

[61] A.M. Wodtke. PhD thesis. University of California, Berkeley, 1986.

[62] A.M. Wodtke, E.J. Hintsa, and Y.T. Lee. "Infrared Multiphoton Dissociation of Three Nitroalkanes". In: J. Phys. Chem. 90.16 (1986), pp. 3549-3558.

[63] A.M. Wodtke et al. "The $\mathrm{Cl}$ to $\mathrm{NCl}$ branching ratio in 248-nm photolysis of chlorine azide". In: Chem. Phys. Lett. 391.4-6 (2004), pp. 334-337.

[64] X. Yang et al. "Universal crossed molecular beams apperatus with synchrotron photoionization mass spectrometric product detection". In: Rev. Sci. Instrum. 68.9 (1997), pp. 3317-3326. 
[65] L.M. Ying et al. "Photodissociation of methylazide: Observation of triplet methylnitrene radical". In: J. Chem. Phys. 105.14 (1996), pp. 5798-5805.

[66] J. Zhang, K. Xu, and G. Amaral. "Ultraviolet photodissociation dynamics of $\mathrm{HN}_{3}$ : the $\mathrm{H}+\mathrm{N}_{3}$ channel". In: Chem. Phys. Lett. 299 (1999), pp. 285-290.

[67] J.Y. Zhang et al. "An experimental and theoretical study of ring closing dynamics in $\mathrm{HN}_{3}$ ". In: Phys. Chem. Chem. Phys. 8.14 (2006), pp. 1690-1696.

[68] P. Zhang, K. Morokuma, and A.M. Wodtke. "High-level ab initio studies of unimolecular dissociation of the ground-state $\mathrm{N}_{3}$ radical". In: J. Chem. Phys. 122.1 (2005), p. 014106. 
Bibliography 
Part II

\section{Design and Construction of a Size Selected Nanoparticle Deposition System}





\section{Chapter 7}

\section{Introduction}

The Department of Dynamics at Surfaces, located at Georg-August-Universität and the Max Planck Institute for Biophysical Chemistry in Göttingen, focuses on developing a deeper understanding of surface chemistry. Its goal is to test new theoretical frameworks for molecule-surface interactions with state-of-the-art experimental techniques and using the results to drive further advancements in the next generation of theory. Up to now, the theory and experiments have focused on interactions of small molecules with model bulk surfaces, such as the NO molecule with $\mathrm{Au}(111)$. These experiments have recently led to the development of an improved theoretical framework for non-adiabatic molecule-surface interactions [7]. As the theory begins to accurately model known experimental results, the need for more sophisticated experiments to challenge the limits of the theory becomes necessary. This creates the need for reliable methods for producing more complex surfaces to be used in these types of experiments.

It has become well known that nanometer sized structures of some materials can possess remarkably different surface chemistry than the bulk surface. A famous example is the highly active catalytic properties of gold nanoparticles in the work of Masatake Haruta in the 1990s [23-25]. Bulk gold has typically been regarded as one of the least reactive metals and a poor catalyst. However, gold nanoparticles can be highly active CO oxidation catalysts. In addition, the activity of nanoparticles varies widely with the size of the particle. Since these discoveries, the field of nanoparticle catalysis has become an incredibly active area of research. Using nanoparticle surfaces is one way of expanding the complexity in dynamics experiments and exploring new molecule-surface interactions.

There are a number of challenges when it comes to creating supported nanoparticle surfaces in a controlled manner. Each combination of nanoparticle-support material can have different interactions. For weakly interacting surfaces, nanoparticles can easily move and aggregate into larger islands or sinter into large particles. Development and characterization of a system for producing controlled nanoparticle surfaces is the central motivation for this part of my dissertation. To meet the wide variety of potential future requirements for collaborative experiments with other instruments in-use in the Dynamics at Surfaces department, the system should be flexible in terms of useable materials and expandability.

To satisfy these goals, magnetron sputtering was chosen as the method for generating nanoparticles. The magnetron sputtering technique produces a large fraction of nanoparticles as ions. This makes it suitable for coupling to traditional ion mass spectrometry meth- 


\section{Chapter 7 Introduction}

ods for selecting a size of nanoparticle to be deposited on the surface. The well established techniques of quadrupole mass spectrometry are utilized to size select the nanoparticles produced in the sputtering source. A number of similar instruments have been reported in the literature in recent years [12, 34, 44, 48, 51, 52]. My goal is to develop and characterize this instrument and provide preliminary results which support its use for future experiments in surface dynamics.

The development of this new instrument for controlled nanoparticle surface deposition will be presented as follows:

Chapter 8 will present an overview of the basic information and theory behind a wide variety of established techniques which have been utilized to create and characterize this system. Chapter 9 is broken into subsections which focus on the specifications of each element of the complete nanoparticle deposition system. This includes the construction details, electronic components, and other capabilities for each system. Chapter 10 presents the results of my thorough characterization of each component and preliminary results supporting the use of this instrument in the planned future experiments. Finally, Chapter 11 summarizes the accomplishments of this dissertation, presents details for three different planned experiments which utilize the system developed here and provides a few options for future expandability. 


\section{Chapter 8}

\section{Fundamentals}

This chapter will cover the basic theory of operation for all the key instruments which work together to produce the size-selected nanoparticle deposition system. The historical development, fundamental physics and equations, advantages and limitations and alternative approaches will be discussed for each of the key elements including: magnetron sputtering, quadrupole mass spectrometry, time of flight mass spectrometry, and surface microscopy techniques.

\subsection{Vacuum Technique}

Basic knowledge of vacuum techniques is essential to the operation of this system. This section will describe some of the fundamental properties of vacuum systems which are necessary for the desired functionality.

\subsubsection{Mean Free Path}

The mean free path, $\lambda$, refers to the mean distance a molecule can travel before collision with another molecule. It can be expressed mathematically as:

$$
\lambda=\frac{1}{n \pi d^{2} \sqrt{2}}
$$

where $n$ is the number density of the molecules and $\pi d^{2}$ is their collisional cross section. The mean free path of Ar molecules at $20^{\circ} \mathrm{C}$ for several pressures is shown in Table 8.1.

The nanoparticle clusters are generated through collisions in a chamber with a pressure on the order of $10^{-1}$ torr. The length of the collision region is variable, but usually around $10 \mathrm{~cm}$. The table shows that there will be a high number of collisions over this distance at this pressure, especially as the cluster size increases. This is essential for the growth of the clusters.

Once the clusters exit the source, it is important that they stop undergoing collisions. Collisions can cause the clusters to fly in all directions, which leads to undesirable losses when trying to transport the cluster beam over $70 \mathrm{~cm}$ to the deposition surface. In these chambers, having a low pressure to transport the beam in a collision free environment becomes essential. A pressure of between $10^{-5}$ to $10^{-6}$ torr is sufficient to expect little to 
Table 8.1: Mean free path of Ar at $20^{\circ} \mathrm{C}$.

\begin{tabular}{cc}
\hline $\begin{array}{c}\text { Pressure } \\
\text { (torr) }\end{array}$ & $\begin{array}{c}\text { Mean Free Path } \\
(\mathrm{cm})\end{array}$ \\
\hline 760 & $7.7 \times 10^{-6}$ \\
$10^{-1}$ & $5.8 \times 10^{-2}$ \\
$10^{-3}$ & 5.8 \\
$10^{-6}$ & $5.8 \times 10^{3}$ \\
$10^{-9}$ & $5.8 \times 10^{6}$ \\
$10^{-12}$ & $5.8 \times 10^{9}$ \\
\hline
\end{tabular}

no collisions over the length of the full instrument. However, the mean free path is not the only consideration when it comes to vacuum in this system.

\subsubsection{Surface Coverage}

The goal of this nanoparticle deposition instrument is to deposit the clusters on surfaces for use in other surface dynamics experiments. To this end, it is important that the observed surface dynamics are being produced by the nanoclusters on the surface and not other contaminants. This makes preserving surface cleanliness an important factor. Table 8.2 shows the time it takes to form a monolayer of $\mathrm{H}_{2} \mathrm{O}$ molecules on a surface for various pressures, assuming a sticking probability of 1 .

Table 8.2: Time to surface monolayer coverage for $\mathrm{H}_{2} \mathrm{O}$ at $20^{\circ} \mathrm{C}$.

\begin{tabular}{cc}
\hline $\begin{array}{c}\text { Pressure } \\
\text { (torr) }\end{array}$ & Time for Monolayer \\
$(\mathrm{s})$
\end{tabular}

Even at a pressure of $10^{-9}$ torr, a monolayer of contaminant will form in under one hour! This leaves little time to deposit nanoclusters and perform experiments after cleaning the surface. For the surface deposition chamber, true UHV pressures will be essential to 
allowing the time to perform future surface dynamics experiments on the nanoparticle substrates.

\subsubsection{Generating and Measuring Vacuum}

There are a wide variety of methods to generate and measure vacuum. I will only focus on the methods used in this nanoparticle deposition system.

- Rough vacuum is attained through the use of standard oil-sealed rotary vane pumps. These are some of the most common pumps available and have a functional range from atmosphere down to around $10^{-3}$ torr. The pump functions by turning an off-center rotor which compresses gas from the inlet and forces out through the exhaust. The oil serves to form a seal in the gap between the rotor vanes and the wall of the pump. These pumps are generally considered to be highly reliable. The biggest drawback is the oil which could potentially end up contaminating the vacuum system. Any contamination by the oil is minimized in this system by the addition of a molecular sieve trap installed at the pump inlet and use of turbomolecular pumps.

- The high vacuum in the system is generated by turbomolecular pumps (TMP) of various sizes and pumping speeds connected to each chamber of the system. Turbomolecular pumps are essentially turbines such as those found in jet engines on airplanes. The rotating blades in a TMP spin at up to 90,000 rotations per minute. The rotors are designed to impart momentum toward the exhaust when they strike gas molecules. They are particularly good for providing a "clean" vacuum as it is very difficult for larger molecules such as pump oil to backstream across the rotors into the vacuum system. A particular modification known as a "compound" or "hybrid" turbomolecular pump is used on the surface deposition and magnetron chambers. In these pumps, an additional molecular drag pumping stage is located below the turbomolecular pump. The main advantage to this design is an increase in the pumping speed for high inlet pressures. This is most important on the magnetron chamber which has to pump against a gas pressure of $10^{-1}$ torr in the source while the system is in operation.

- Rough vacuum is measured by Pirani gauges which are a type of thermal-conductivity gauge. The operating principle is based on the thermal conductivity of a gas decreasing as the pressure of the gas decreases. In thermal-conductivity gauges, a wire filament is electrically heated by running an electrical current through it. The temperature of the filament will depend on the rate of heat transfer to the surrounding gas, which will decrease with the pressure. The resistivity of the wire changes with temperature. This filament is part of a Whetstone bridge in a Pirani gauge. The varying resistance of the filament causes a voltage imbalance in the bridge circuit which is what provides an indication of the pressure in the system. These types of gauges are not reliable below $10^{-3}$ torr as the thermal conductivity becomes essentially zero at this point and no further change can be measured. 
- High vacuum is measured by ionization gauges. In an ionization gauge, electrons are produced by thermionic emission from a hot filament and ionize nearby gas molecules by electron impact. These ions are then collected on a biased electrode. The current which is measured by the electrode is proportional to the number of ions which are collected on it which is proportional to the pressure. A common configuration of the ionization gauge was designed by Bayard and Alpert [3] and this is the design which is used to measure the pressure in each chamber of the nanoparticle deposition system.

\subsubsection{Summary of Vacuum in Relation to Nanoparticle Deposition System}

Summarizing the impact vacuum has on the design of the nanoparticle deposition system:

- A vacuum below $10^{-5}$ torr is necessary across the system to maintain the quality of the cluster beam generated in the source. A pressure higher than this would lead to undesirable losses due to collisions.

- The surface chamber should be at or below $10^{-9}$ torr in order to allow for surface dynamics experiments to be performed on clean nanoparticle deposited surfaces.

- To achieve these pressures, standard good vacuum practices are employed through the system. The chambers and components are assembled with the ConFlat system of flanges and copper gaskets. Chambers are made from stainless steel, cleaned with alcohol, and handled while wearing gloves to protect from fingerprints inside the system. Only materials with low outgassing, suitable for use in vacuum are chosen, such as Kapton coated wire and alumina ceramics.

\subsection{Magnetron Sputtering}

One of the most important decisions in designing the nanoparticle deposition system is the method by which the nanoparticles are generated. There are a number of techniques available and routinely used for producing cluster beams for surface deposition including laser ablation, thermal evaporation, sputtering and others. Among these methods, magnetron sputtering has significant advantages for meeting the desired functionality of this nanoparticle deposition system. This section will cover the development history of the magnetron source and describe the basic dynamics of cluster growth and ion formation in this type of source.

\subsubsection{Development of the Magnetron Sputtering Source}

While sputtering deposition techniques have existed for quite some time, the use of a magnetron discharge in sputtering sources is a relatively recent development. "Glow discharges" 
were first conceptualized by Penning [43] around the 1930s, and have been gradually improved over the decades. The magnetron-type discharge source was first implemented and used for cluster deposition by Hellmut Haberland in the early 1990s [17-21]. Since then, the technique has been adapted into a number of commercially available systems and is widely used to produce thin films for industrial applications.

\subsubsection{Operating Principles}

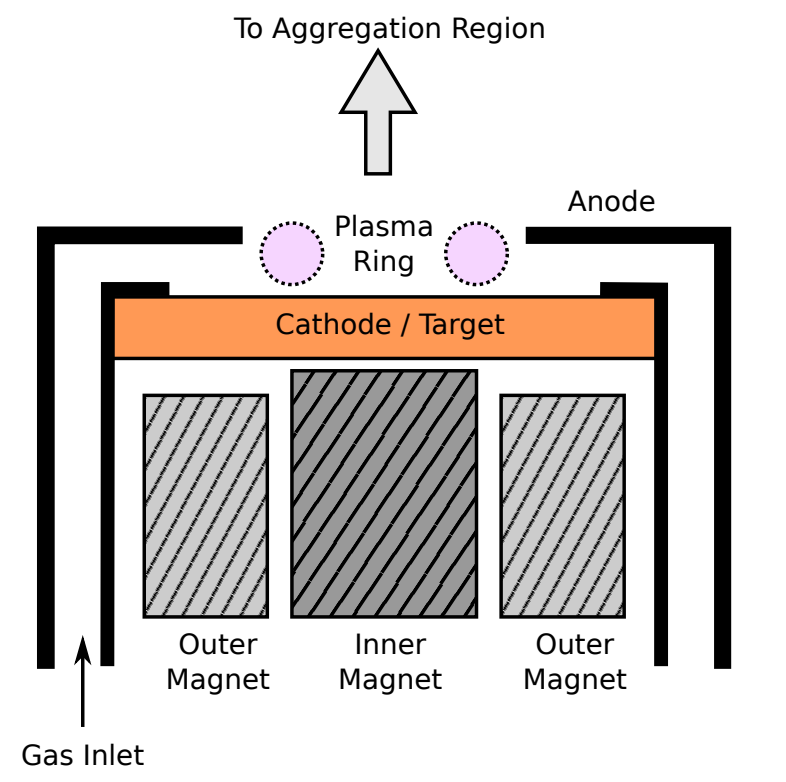

Figure 8.1: Schematic cross section drawing of the head portion of a magnetron. The sputtering gas flows in between the grounded outer anode and the biased cathode and a plasma discharge is created. The electrons in this plasma are confined to a ring by the magnetic field created by a gap in two concentric cylindrical magnets.

The head of the magnetron sputtering source is designed as seen in Figure 8.1. Two concentric, cylindrical magnets create a permenant magnetic field with a maximum strength in the gap between them. In this design, electrons are trapped in the ring of maximum magnetic field strength. The sputtering target is located directly above the magnets. A grounded metal shield sits 0.2 to $0.3 \mathrm{~mm}$ above the edge of the target surface. A rare gas, termed the sputtering gas, can flow from in between this gap directly above the target surface. The sputtering gas is typically argon. A potential is applied across the gap between the shield (the anode) and the sputtering target (the cathode). This voltage creates a discharge which initiates the formation of a plasma from the argon gas. The electrons in the plasma are confined directly above the target in the magnetic field ring. Argon ions 


\section{Chapter 8 Fundamentals}

formed in the plasma are driven toward the cathode by the electric field and when they strike with enough energy, metal atoms can be sputtered from the target's surface. Some these metal atoms are carried by the flow of argon gas away from the sputtering target through an aggregation region where clusters can form. Further details of the dynamics of cluster formation and growth will be discussed in the next subsection. Due to the plasma being confined to a narrow ring above the metal target, the majority of sputtering occurs around this ring, leading to a large fraction of the material in the center and edge of the target being underutilized.

\subsubsection{Cluster Growth Mechanisms}

The cluster growth dynamics in a magnetron sputtering source are similar to other types of gas aggregation sources, of which many studies have been published [11, 13, 29, 30, 33, $37,50]$.

Metal atoms are sputtered from the target surface by impact of argon ions. These metal atoms can produce a dimer "seed" cluster through a three-body collision process with a rare gas atom.

$$
\mathrm{M}+\mathrm{M}+\mathrm{G} \longrightarrow \mathrm{M}_{2}+\mathrm{G}^{*}
$$

(Nucleation)

The three-body process with a rare gas atom is required to create the dimer due to momentum and energy conservation laws. The gas atom takes the excess energy released in the dimer formation. Once the initial dimer seed is created, other free metal atoms can condense with it, forming a larger cluster one atom at a time.

$$
\mathrm{M}_{\mathrm{n}}+\mathrm{M} \longrightarrow \mathrm{M}_{\mathrm{n}+1}^{*}
$$

(Condensation)

This process creates a more energetic cluster than the parent cluster and the growing clusters need to be cooled through further collisions with rare gas molecules, otherwise the competing vaporization process to lose metal atoms becomes more favorable. Another process can occur once there is a sufficient density of growing clusters - two growing clusters can coalesce into one larger one.

$$
\mathrm{M}_{\mathrm{n}}+\mathrm{M}_{\mathrm{m}} \longrightarrow \mathrm{M}_{\mathrm{n}+\mathrm{m}}
$$

(Coalescence)

\subsubsection{Ion Formation Mechanisms}

An interesting characteristic of magnetron sputtering is the high fraction of ionized clusters which are produced natively without any need for a secondary ionization method. Several processes by which ions can be created in the magnetron discharge have been proposed [21]. 


\section{Cationic Clusters}

For cationic clusters, there are three formation mechanisms. While most of the directly sputtered metal atoms are neutral, about $0.1 \%$ is positively charged. When this charged ion is incorporated into a cluster, the resulting cluster is charged. In addition to metal ions being formed directly in the sputtering, there is a large amount of $\mathrm{Ar}^{+}$ions present. Collisions between a metal cluster and an argon ion can result in a charge transfer from the argon to the cluster. The last mechanism to produce cationic clusters is known as Penning ionization. In this process, an argon atom can be electronically excited by interactions with the electrons in the plasma discharge. This excited state argon atom can collide with a metal cluster, transferring its excess energy and ejecting an electron from the metal cluster. A summary of these processes is as follows:

$$
\begin{array}{lr}
\mathrm{M}_{\mathrm{n}}+\mathrm{M}^{+} \longrightarrow \mathrm{M}_{\mathrm{n}+1}^{+} & \text {(Ion Formed in Sputtering) } \\
\mathrm{Ar}^{+}+\mathrm{M}_{\mathrm{n}} \longrightarrow \mathrm{Ar}+\mathrm{M}_{\mathrm{n}}^{+} & \text {(Charge Transfer) } \\
\mathrm{Ar}^{*}+\mathrm{M}_{\mathrm{n}} \longrightarrow \mathrm{Ar}+\mathrm{M}_{\mathrm{n}}^{+}+\mathrm{e}^{-} & \text {(Penning ionization) }
\end{array}
$$

\section{Anionic Clusters}

A high fraction of anionic clusters are produced in magnetron sputtering as well. The mechanism for anion formation is mainly due to the high density of electrons confined above the metal surface by the magnetron field where there is the possibility of an electron attaching to a metal cluster.

$$
\mathrm{M}_{\mathrm{n}}+\mathrm{e}^{-} \longrightarrow \mathrm{M}_{\mathrm{n}}^{-}
$$

(Electron Attachment)

\section{Neutral Clusters}

With the variety of mechanisms for producing charged species, it might be expected that almost all the clusters produced in magnetron sputtering are charged. While there are a number of mechanisms for producing charged clusters, there are also a number of ways charged clusters can be neutralized before exiting the growth region, such as free electrons neutralizing cationic clusters.

\section{Distribution of Charged Species}

The fraction of charged species exiting the source varies by the type of metal being sputtered, but can be between 30 and 80 percent of the produced clusters [19]. This is already higher than what is typically produced through other ionization methods, making the cluster beam created through the magnetron sputtering method well suited to mass separation using traditional ion mass spectrometry techniques. 


\subsubsection{Shape of Mass Distribution}

The particle size distribution of clusters formed in an inert gas aggregation source, such as the magnetron sputtering source, typically resembles a log-normal distribution. This has mostly been an empirical observation, though there are also numerical calculations which derive the same shape [4, 14, 40]. A complete treatment of why the distributions observed follow this form is beyond the scope of this dissertation and further discussion can be found in the references. The log-normal distribution function is described by the equation:

$$
f(x ; \mu, \sigma)=\frac{1}{x \sigma \sqrt{2 \pi}} \exp \left(-\frac{(\ln x-\mu)^{2}}{2 \sigma^{2}}\right)
$$

where $x$ is a mass, $\mu$ is the mean of the mass distribution and $\sigma$ the standard deviation. This equation is used to fit the observed nanoparticle mass distribution functions in the characterization of the source in Chapter 10.

\subsubsection{Calculating Nanoparticle Size from Mass}

Size selecting particles with this instrument is done by filtering according to the mass of the particle. It is be convenient to be able to relate the mass of the particle to an expected size. This can be done by using a spherical particle approximation. Assuming the particle's lowest energy form will be a homogenous sphere, the expected size can be calculated by the formula for the volume of a sphere:

$$
V=\frac{4}{3} \pi r^{3}
$$

A further assumption must be made to estimate the volume of the particle from the mass. The volume can be estimated from the density, $\rho$, of the particle and the mass:

$$
V=\frac{m}{\rho}
$$

Combining these equations, the diameter of a particle as a function of its mass is determined by the equation:

$$
d=2 \sqrt[3]{\frac{3 m}{4 \rho \pi}}
$$

The expected size calculated by this approximation will be referred to throughout the characterization of deposited particles in Chapter 10. 


\subsubsection{Advantages and Disadvantages of Magnetron Sputtering} Advantages

Magnetron sputtering offers a number of compelling advantages and these characteristics make it the ideal source to achieve the goals for the nanoparticle deposition system. The most important advantages include:

- This technique produces a high fraction of native ions with no need for external ionization sources.

- Functions with a wide variety of target materials which are easily available commercially. The DC sputtering employed in this instrument requires conducting materials like metals. RF sputtering can expand the target materials to semiconducting and insulating materials. The flexibility in material choices will allow for the system to be used for more experiments in the future, beyond the initial goals laid out.

- Target material can be easily changed from day-to-day. The mechanisms of cluster growth and ionization are universal so nanoparticles of different materials can be produced with the same instrumentation. Many wet synthesis methods can be used for producing size selected nanoparticles, but the recipes cannot be used with every material, giving the sputtering source an advantage.

- Reactive sputtering is possible. Leaking a flow of oxygen or nitrogen into the sputtering source can produce metal oxide or nitride nanoparticles directly. This also provides flexibility for future experiments.

- Targets of more than one material can be used to produce bimetallic nanoparticles. This can have interesting applications in catalysis.

- The particle size distribution can be broadly controlled with the source parameters before performing any mass or size selection techniques.

- Nanoparticles generated through gas condensation sources can be deposited cleanly and free from significant contamination. Chemical methods often require encapsulation by organic ligand complexes to produce size selection, but these ligands can interfere with proposed experiments, making a clean size selection method desirable.

\section{Disadvantages}

The magnetron sputtering source, of course, also has some drawbacks which need to be worked around. The chief disadvantages include:

- Sputtering and aggregation is a dynamic system which can be prone to both sudden changes in the size distribution and slow drifts over time. This can make it difficult to reproduce an exact size distribution from day-to-day with the same conditions. 
- There is a lack of precise control over size distributions generated with source conditions.

- This method is not well suited to producing very small clusters such as dimers and trimers.

- The upper limit on the size of the particles which can be produced is low. Sizes greater than $10 \mathrm{~nm}$ are not very practical using this method.

- Despite significant ionization, a large fraction of neutral clusters are still produced. These cannot be mass selected and will remain in the beam following mass selection of the ion fraction. The ionic portion must be deposited off-axis to avoid contamination by unwanted sizes of neutrals.

- The source requires frequent maintenance to remove thick deposits of clusters around apertures and the sputtering chamber components.

- Not all materials can be used. Low melting point metals are problematic for this source. Highly magnetic materials interfere with the operation. Brittle materials often do not sputter efficiently.

\subsection{Quadrupole Mass Spectrometry}

Quadrupole Mass Spectrometry (QMS) is the method chosen to produce size selection of the ionic portion of nanoparticles which are produced in the magnetron sputtering source. This section will cover the background information and mathematical formulation of QMS. Due to the wide spread implementation of this instrument, a number of good review articles are available for more information $[9,10,41]$.

\subsubsection{Development of Quadrupole Mass Spectrometers}

The linear quadrupole mass spectrometer was first introduced by Wolfgang Paul and coworkers in the late 1950s while at the University of Bonn [42].

The linear quadrupole consists of two pairs of parallel hyperbolic electrodes with potentials that have both a DC and time varying RF voltage component. The potentials on each pair of rods have the same magnitude but opposite polarity. The hyperbolic electrodes are often approximated by circular rods due to the difficulty of precision manufacturing the perfect hyperbolic surfaces.

Due to its low cost and ease of operation for the end user, the quadrupole has become the most widely used instrument in mass spectrometry. Later innovations led to the construction of three dimensional quadrupoles for ion trapping and higher order multipoles for applications such as ion guides. 


\subsubsection{Operating Principles}

The electric field potential of the quadrupole can be described in cartesian coordinates by the following equation:

$$
\phi(x, y)=\left(\frac{x^{2}-y^{2}}{r_{0}^{2}}\right) \phi_{0}
$$

The electric potential applied to the electrodes is $\phi_{0}$. In this description, the electrode surfaces are spaced at a distance of $r_{0}$ from the center axis position where $x=0$ and $y=0$. It is readily apparent from this equation that the potential at this position is zero.

If the quadrupole potential were static, no filtration would be produced the charged ion entering the quadrupole would simply be attracted to the oppositely charged pair of electrodes and collide with the electrode. This is solved by applying a potential with both a $\mathrm{DC}$ component and a time varying RF component. The equation for this potential applied to the quadrupole electrodes is:

$$
\phi_{0}(t)=U-V \cos (\omega t)
$$

where $U$ is the DC voltage and $V$ is the RF voltage with the driving frequency $\omega$. This time varying potential can be substituted into the previous equation for the quadrupole's electric field potential to produce:

$$
\phi(x, y, t)=\left(\frac{x^{2}-y^{2}}{r_{0}^{2}}\right)(U-V \cos (\omega t))
$$

This now time varying electric field alternates between attractive and repulsive forces on the rods allowing for ions with the right set of characteristics to have stable oscillation trajectories through the quadrupole.

To determine which ions have stable trajectories, the motion of the ions in this field must be described. The starting point for the description of ion motion comes from the force the ions experience which can be described by Newton's Second Law and a form of the Lorentz Force Law neglecting the magnetic field contribution:

$$
F=m a=-e \nabla \phi(x, y, t)
$$

Separating this into $x$ and $y$ components and replacing acceleration by the equivalent second derivative of position with time gives the following:

$$
\begin{aligned}
& F_{x}=m \frac{\partial^{2} x}{\partial t^{2}}=-\frac{2 e x}{r_{0}^{2}}(U-V \cos (\omega t)) \\
& F_{y}=m \frac{\partial^{2} y}{\partial t^{2}}=+\frac{2 e y}{r_{0}^{2}}(U-V \cos (\omega t))
\end{aligned}
$$


These equations can be rearranged into the following differential equations:

$$
\begin{aligned}
& m \frac{\partial^{2} x}{\partial t^{2}}+\frac{2 e x}{r_{0}^{2}}(U-V \cos (\omega t))=0 \\
& m \frac{\partial^{2} y}{\partial t^{2}}-\frac{2 e y}{r_{0}^{2}}(U-V \cos (\omega t))=0
\end{aligned}
$$

Which can be simplified to:

$$
\begin{aligned}
& \frac{\partial^{2} x}{\partial t^{2}}+(a+2 q \cos (2 \tau)) x=0 \\
& \frac{\partial^{2} y}{\partial t^{2}}-(a+2 q \cos (2 \tau)) y=0
\end{aligned}
$$

with the new variables a, $\mathrm{q}$ and $\tau$ being defined as:

$$
\begin{aligned}
a & =\frac{4 e U}{m r_{0}^{2} \omega^{2}} \\
q & =\frac{2 e V}{m r_{0}^{2} \omega^{2}} \\
\tau & =\frac{\omega t}{2}
\end{aligned}
$$

Equations 8.14 and 8.15 are the differential equations for ion motion in the $x$ and $y$ dimensions and they have a form known as the Mathieu equation.

\subsubsection{Mathieu's Equation and the Quadrupole Stability Region}

Mathieu's equation is a specialized case of the Hill Equation which can be applied to ion motion in an oscillating electric field [35]. The solution to the Mathieu equation provides boundary conditions for ion trajectories which are stable in the quadrupole mass filter. An ion's trajectory is considered stable if the limit of the amplitude of oscillation is finite as time approaches infinity. Figure 8.2 shows a sets of parameters $(a, q)$ for which these equations produce stable trajectories. The shaded regions are stable in either the $x$ or $y$ dimension. As can be seen, only very narrow regions of overlap between the two are simultaneously stable in both directions. These are the valid operating regions of the quadrupole mass filter. There are technically an infinite number of solutions which produce stability regions, however the voltage and frequency combinations required to access the higher order stability regions are so prohibitive that most quadrupoles only operate inside the first stability region. An enlarged plot of the Mathieu stability diagram showing the first stability region is displayed in Figure 8.3.

In operation, $U$ and $V$ are kept at a constant ratio. For a given frequency, sweeping the both voltages while keeping $U / V$ constant will produce a "scan line" where masses are subsequently brought into this stability region. Two scan lines are represented in Figure 8.3 


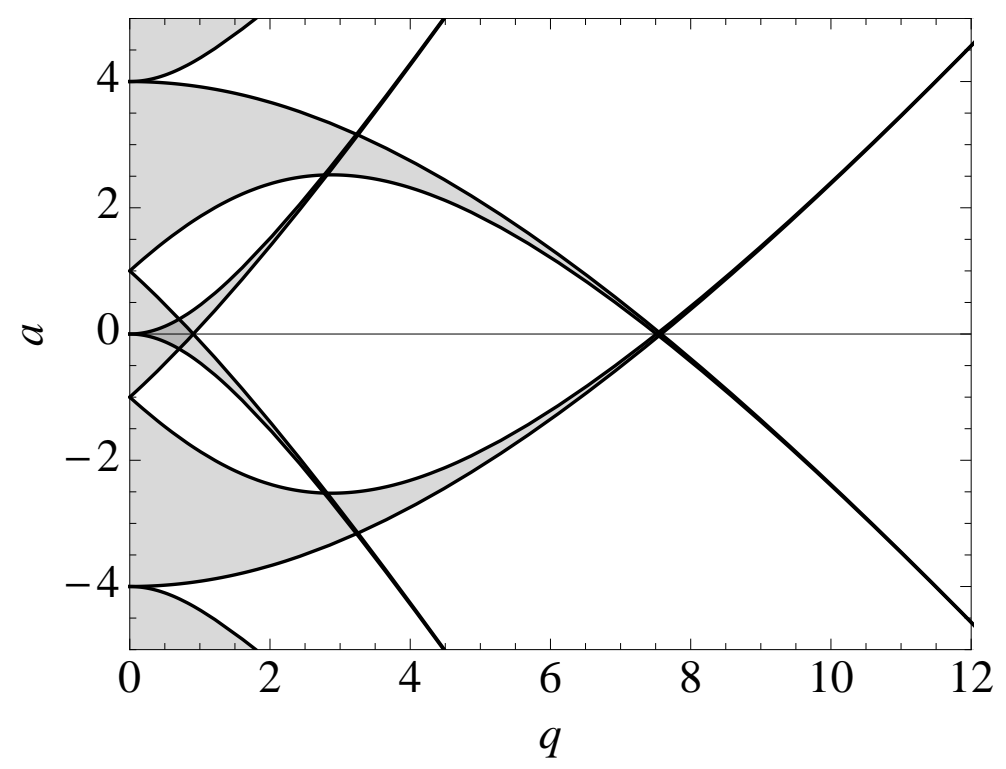

Figure 8.2: Mathieu Stability Diagram. The shaded areas describe regions where either the $x$-trajectories or $y$-trajectories are stable. Only the darker areas where these regions overlap will allow stable transmission in the quadrupole.

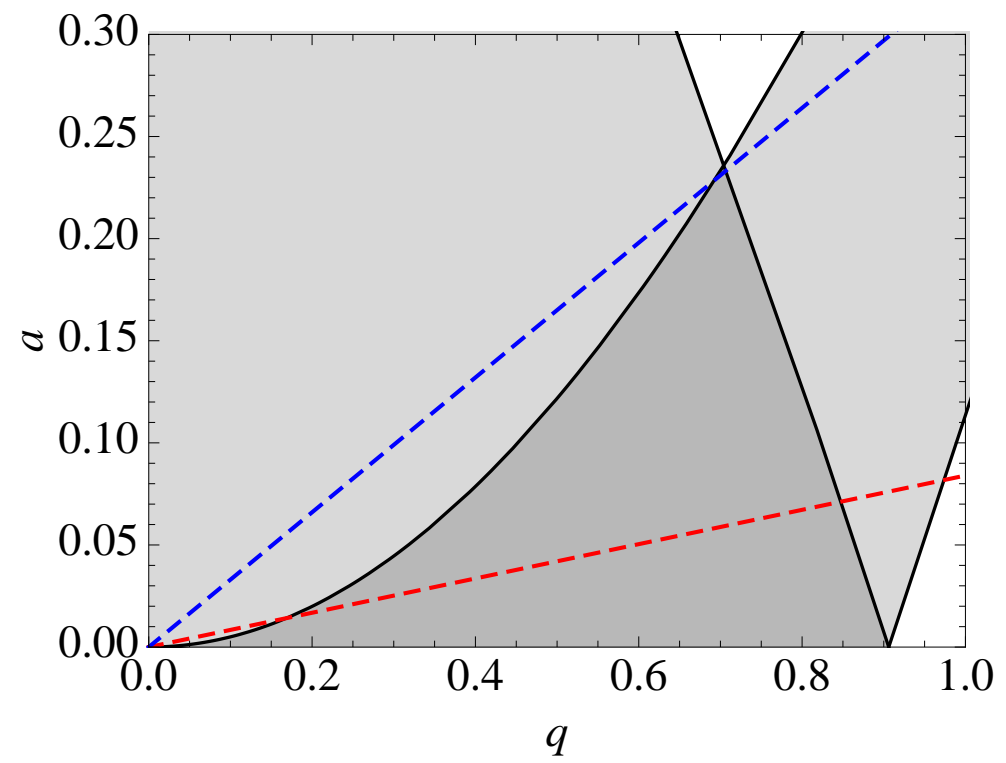

Figure 8.3: Enlarged view of first stability region of Mathieu Diagram in figure 8.2. The darker region of overlap between the two lighter areas describes the region of ion motion which is stable in both the $x$ and $y$ dimensions. The dashed red line shows a scan line with $100 \%$ mass resolution and the dashed blue line shows a scan line with $2 \%$ mass resolution. 


\section{Chapter 8 Fundamentals}

by dashed lines. The blue dashed line has a $U / V$ of 0.165 . The range of this scan line which passes through the stability region is narrow and corresponds to a mass resolution of $2 \%$ or a resolving power of 50 . The red line has a $U / V$ of 0.042 . A much wider range of masses are inside the stability region for this line and this corresponds to a mass resolution of $100 \%$ or resolving power of 1 .

If no DC voltage is applied ( $U=0$ and the scan line is a horizontal line on the $x$ axis) then the quadrupole acts as an ion guide which transmits all ions without mass discrimination.

There are a number of other factors which can affect transmission through the quadrupole, such as the acceptance and emittance window for ions and effects of fringe fields at the exit of the quadrupole, which are outside the scope of information necessary for the dissertation work performed here. However, improvements in some of these areas could lead to improvements in the transmission of ions in future modifications to the system.

\subsubsection{Equations of Transmitted Mass and Mass Resolution}

The mass passed in a quadrupole can be calculated from the formula:

$$
m=7 \times 10^{7} \frac{V}{f^{2} d^{2}}
$$

where $d$ is the diameter of the rods in meters, and $V$ is the RF potential in volts with frequency $f$ in hertz.

The theoretically best resolution achievable by the quadrupole can be calculated from the formula:

$$
\Delta m=4 \times 10^{9} \frac{V_{z}}{f^{2} L^{2}}
$$

where $\mathrm{V}_{z}$ is the kinetic energy of the ion in volts, and $L$ is the length of the quadrupole in meters. Typical quadrupoles may have rods of a few millimeters in diameter and operate with frequencies around $1 \mathrm{MHz}$ which gives an operating range of up to several hundred amu when used with reasonable voltages. For example, for $6 \mathrm{~mm}$ rods, and $100 \mathrm{~V}$ AC voltage at 1.0 MHz, the mass filtered is calculated to be $194 \mathrm{amu}$. For rods which are $10 \mathrm{~cm}$ long and ion kinetic energies of $10 \mathrm{~V}$, the optimum resolution of these ions is calculated at $4 \mathrm{amu}$.

\subsubsection{Use of Quadrupoles for Filtering High Masses}

The ionic nanoparticles produced in the magnetron sputtering source have masses well above the operating range of the typical quadrupole mass filter. It would require around 100,000 volts at $1.0 \mathrm{MHz}$ to filter clusters with a mass of 194,000 amu for a quadrupole with the characteristics described in the previous section. However, by running with much lower frequencies on larger rods, it is possible to extend the mass range dramatically, though making some sacrifices to the ultimate resolution and transmission. This type of operation was developed and implemented by Prof. Chris Binns at the University of Leicester in the late 1990s specifically to work with nanoparticle cluster sources. 
By dropping the frequencies to $1-100 \mathrm{kHz}$ and expanding the rod size to $25 \mathrm{~mm}$, the operating range of the quadrupole can be very large. For example, with $200 \mathrm{~V}$ AC at $1 \mathrm{kHz}$, the mass selected would be $22 \times 10^{6} \mathrm{amu}$ and for $10 \mathrm{~V}$ at $100 \mathrm{kHz}$ the selected mass would be just 112 amu. While this range seems incredible, keep in mind there is a severe trade-off in resolution. For the mass $112 \mathrm{amu}$ with $10 \mathrm{~cm}$ long rods, the optimal $\Delta m$ is now $200 \mathrm{amu}$ - almost double the mass being selected!

By choosing to expand the quadrupole's operating range to accommodate the wide mass range of nanoparticles produced in the magnetron source, one gives up the ability to individually resolve small clusters with this instrument.

\subsubsection{Advantages and Disadvantages of Mass Filtering with the QMF}

Aside from the general low cost and ease of use which is common to most quadrupoles, the two biggest advantages are the ability to produce continuous, stable mass filtering and the usability over a wide mass range.

The magnetron sputtering source produces a continuous beam of ionic clusters and many mass filtration techniques, such as time of flight, operate with pulses which would throw away a large portion of otherwise usable ions. With the quadrupole, the ion beam is kept continuous from production to deposition.

The second advantage is the wide range of masses that can be selected with the modifications developed by $\mathrm{C}$. Binns. This allows for tuning the quadrupole to select masses anywhere between 30 and $3 \times 10^{6}$ amu with this instrument's parameters, which allows for a wide range of cluster sizes to be selected.

There are drawbacks to choosing the quadrupole filter as well. The biggest disadvantages are the mass resolution, transmission efficiency, and divergence of the quadrupole. In order to attain the wide mass range, the resolution of the quadrupole must be sacrificed. Theoretically, it is still possible to operate near the tip of the stability region, however the ion transmission window becomes so small that it is not usually practical to work at this point and have usable ion intensities. In addition, the oscillatory motion of the ions produced by the quadrupole makes for a poorly behaved beam at the exit which requires additional effort to bring to the deposition surface. It will be shown in Chapter 10 that without ion optics, no ion beam reaches the deposition surfaces, likely due to the divergent nature of the ions after filtering, or perhaps caused by fringe electric fields at the exit to the quadrupole.

\subsection{Time of Flight Mass Spectrometry}

Time of Flight Mass Spectrometry (TOFMS) plays an important role in the characterization of the size selection capabilities of the complete system. A home-built time of flight mass analyzer was made for this specific purpose. This section will describe the basic concepts in TOFMS. A thorough review of TOFMS is available in Reference [15]. 


\subsubsection{Overview of Time of Flight Mass Spectrometry}

A simple operating diagram of a general time of flight mass spectrometer can be seen in Figure 8.4. Ions enter the spectrometer while no electric field is applied. At some starting time, $t=0$, an electric field pulse is applied to drive the ions toward the detector. The ions are accelerated in the pulse region, then pass through a mesh into a field-free drift tube. The drift region is typically very long and can be over a meter in length. At the end of the drift region, ions hit a detector and their arrival times are recorded. Low mass ions attain higher velocities from the extraction pulse than high mass ions, and therefore arrive at the detector earlier.

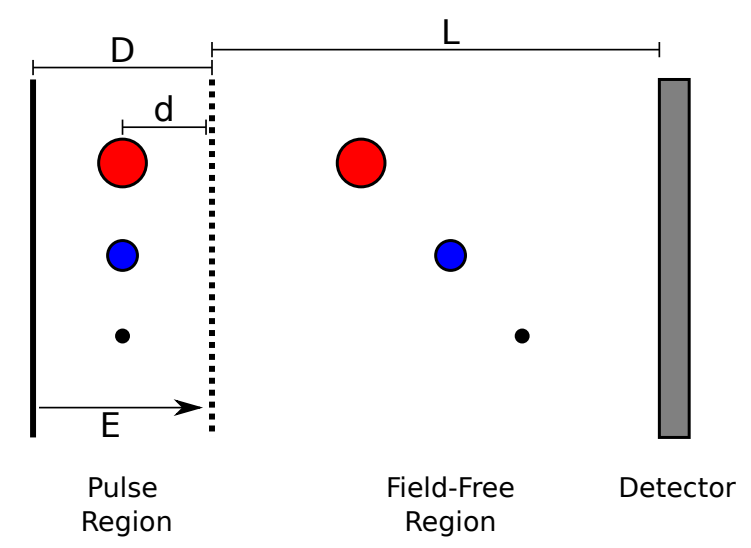

Figure 8.4: Drawing depicting operation and the relevant variables of a single extraction stage time of flight instrument.

There are two regions which need to be mathematically described in similar yet separate ways. In the extraction region, ions undergo acceleration toward the detector and the amount of time they spend in the extraction pulse will vary by the mass of the ion. In the field-free region, there is no longer any acceleration and the velocity an ion has is fixed from the acceleration in the previous region. These statements are only valid for an electric pulse which is not switched off until all ions have exited the extraction region.

The time of flight scales predictably with the square root of the mass once the instrument has been properly calibrated with known mass standards. This then allows the conversion of arbitrary arrival times to masses.

\subsubsection{Fundamental Physics and Equations}

As with the formulation for ion motion in the quadrupole, the time of flight of an ion can be described starting from Newton's Second Law and the Lorentz Force Law.

$$
F=m a
$$




$$
F=e E
$$

The electric field, $E$, can be related to the voltage applied to the extraction plate, $U$, and the distance between plates in the extraction region, $D$, by:

$$
E=\frac{U}{D}
$$

Combining these three equations gives:

$$
m a=\frac{e U}{D}
$$

Integrating this equation twice and solving for the time it takes to span the distance, $d$, to exit the extraction region results in a time in the extraction region of:

$$
t_{\text {extraction }}=\sqrt{\frac{2 d m D}{e U}}
$$

At this point, the ions have exited the extraction region into the field-free region. The velocity an ion has in the field-free region can be found from its kinetic energy:

$$
E_{T}=\frac{1}{2} m v^{2}
$$

In moving through the electric field, the ion gains a kinetic energy proportional to the distance it travels in the electric field:

$$
E_{T}=e d E=\frac{e d U}{D}
$$

Setting the expressions for kinetic energy equal and solving for $v$ gives:

$$
v=\sqrt{\frac{2 d e U}{m D}}
$$

Since this velocity is constant in the field-free region, the time it takes an ion with the velocity $v$ to travel the distance $L$ can be found simply by:

$$
t_{\text {fieldfree }}=\frac{L}{v}
$$

And replacing the expression for $v$ into this equation produces the equation for the time of flight in the field-free region:

$$
t_{\text {fieldfree }}=L \sqrt{\frac{m D}{2 d e U}}
$$


The total time of flight from $t=0$ at the beginning of the electric pulse until the time the ion hits the detector at the end of the field-free region is then the sum of extraction and field-free flight times:

$$
t_{\text {total }}=t_{\text {extraction }}+t_{\text {fieldfree }}=\sqrt{\frac{2 d m D}{e U}}+L \sqrt{\frac{m D}{2 d e U}}
$$

As can be seen from this equation, the total time of flight is proportional to $\sqrt{m / e}$. This is an important point which I will come back to in the characterization of the time of flight mass analyzer in Chapter 10.

\subsubsection{Further Time of Flight Techniques}

There are a number of more advanced methods in time of flight mass spectrometry which are not used in this dissertation work. The mathematical descriptions above are for an ideal case where the ion beam has no spread in starting position and no initial velocities in the time of flight direction. In the practice, the width of the ion beam and variation in the kinetic energy of the ions due to initial velocities will degrade the ability to resolve individual masses. The spread in initial position can be accounted for by using a two stage extraction which allows for adjustment of the space focusing position [57]. Further improvements can be made with use of a reflectron time of flight mass spectrometer, which uses an electrostatic mirror to essentially double the flight distance and correct for variations in initial kinetic energy.

\subsection{Surface Analysis Techniques}

To determine if the deposition system is successful, it is necessary to evaluate the nanoparticle covered surfaces from several perspectives. There are two microscopy techniques I relied on to examine the nanoparticle substrates. Atomic Force Microscopy (AFM) is very sensitive to determining the height of particles on the surface and is good for determining statistical size distributions. Transmission Electron Microscopy (TEM) is a powerful analysis technique which can even determine the lattice spacing in the clusters under some conditions, allowing identification of specific crystal structures.

\subsubsection{Atomic Force Microscopy}

Atomic Force Microscopy (AFM) is a type of Scanning Probe Microscopy (SPM) which produces topographical maps of surfaces with very high (sub-nanometer) resolution in the height dimension. On an atomically flat substrate, this allows for high resolution topographical imaging of deposited nanoparticles. This section will introduce the basic operation of the atomic force microscope and the general properties of an AFM image. 


\section{Basic Concept}

A simplified schematic of the operation of an atomic force microscope is shown in Figure 8.5. A laser is focused on the end of a cantilever. The cantilever has a tip which can be on the order of just a few nanometers at the sharpest point. This cantilever tip is brought into contact with the surface. Piezoelectrically driven motors raster the tip back and forth across the surface. As the tip encounters features with different heights on the surface, the cantilever moves up and down, the laser reflecting off the cantilever is deflected and tiny changes in the height can be measured with a photodiode detector. These deflections on the photodiode as a function of cantilever position are converted with the aid of computer software into images that are topographical maps of the surface.

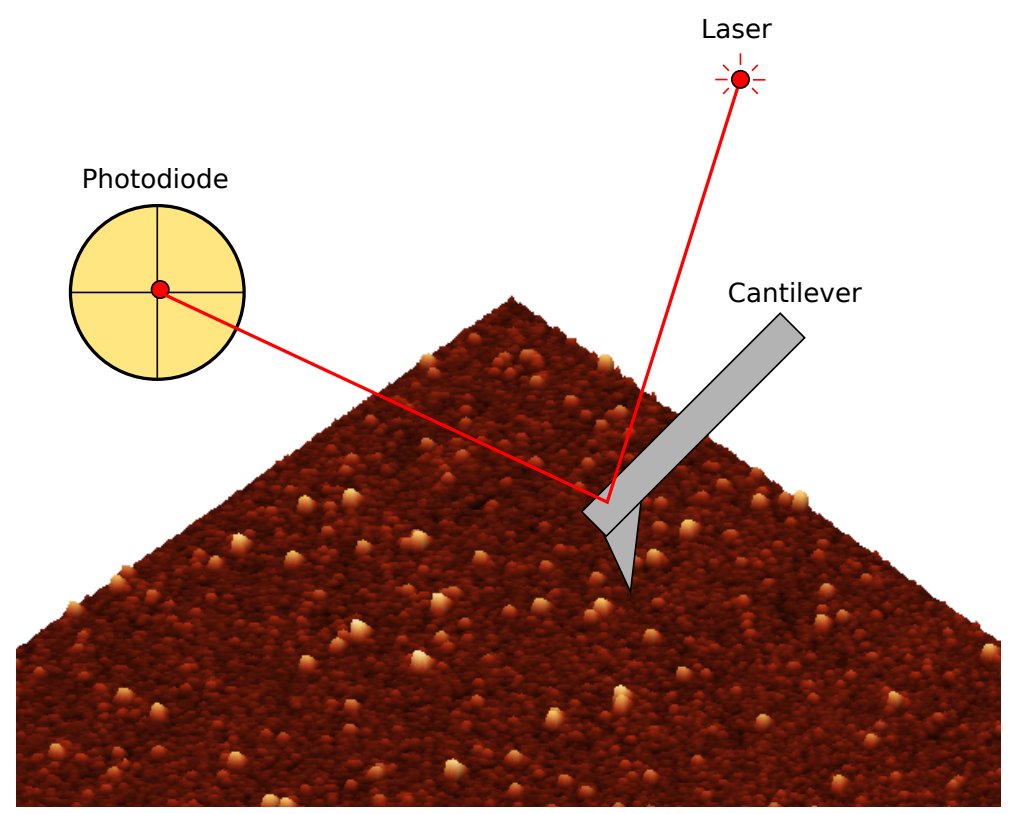

Figure 8.5: Diagram depicting the operation of a typical AFM system. A laser is reflected off the back of a cantilever onto a position sensitive photodiode. The cantilever moves across the surface producing deflections in the laser position which is recorded.

The AFM is extremely sensitive to the height of objects on the surface. However, the $x-y$ dimensions are convoluted due to the size of the tip. It is possible, if the tip size and shape is well known, to de-convolute the $x-y$ dimension to attain more accurate information, however most of the time, only the height information is used.

\section{Dynamic Contact Mode}

A common improvement over the basic "contact" mode of operation described above is called "dynamic contact" or "tapping" mode. In this mode, the cantilever is instead oscillated near its resonant frequency and brought close to the surface. As the tip is scanned across the surface, deflections due to surface height changes are still measured, but the tip 


\section{Chapter 8 Fundamentals}

only spends a fraction of time interacting with the surface. In contact mode, the tip can produce changes on the surface due to the force it exerts, such as dragging a nanoparticle across the surface. While this can be desirable in some applications (intentionally moving and arranging nanoscale objects or nanolithography), it is not usually desirable for analytical purposes. Dynamic contact is generally an improvement over contact mode in all aspects and also contains more information, for example from measuring the changes to the oscillation phase or frequency of the cantilever while scanning. All of the AFM scans which are reported in this dissertation were acquired in a dynamic contact mode.

\section{Advantages and Disadvantages of AFM}

The advantages and main reasons for using atomic force microscopy for characterizing deposited nanoparticles include:

- A high resolution in the height measurement. AFM is capable of very accurate measurement of the topography of a sample when the instrument is operated properly.

- Wide viewing areas relative to transmission electron microscopy. Scans can easily be done for several micron areas which can provide the height information for a statistically relevant number of nanoparticles in just a single scan.

In addition, the AFM is useful for my characterization due to the access and availability of instrumentation. The AFM can be accessed on short notice on a day-to-day basis. This is not the case with other instruments. The transmission electron microscope requires prior scheduling and there can be a high demand for time on it.

The disadvantages of AFM in this characterization work include:

- This technique requires atmospheric exposure of the substrate. Most atomic force microscopes are bench-top systems which requires the samples to be exposed to air. This can lead to contamination of the nanoparticle samples from the environment.

- There are notable inconsistencies in height information for nanoparticle samples. As will be further discussed in Chapter 10, there have been observations of accuracy distorting capillary interactions between tip and nanoparticle samples. The deflections produced by these capillary effects can lead to an unaccountable error in the measured heights.

- The time required for a single scan. At scan rates which produce high quality images, scan times can easily be over 15 minutes for just one image.

- Replacing tips or degradation in the tip quality over time can affect the information obtained in the image.

- There are many common imaging artifacts which can mislead an uninformed microscopist. 


\subsubsection{Transmission Electron Microscopy}

Transmission Electron Microscopy (TEM) is the second microscopy method used to obtain useful information to characterize the performance of the nanoparticle deposition system. TEM is a complex subject and a detailed treatment of all the topics which go into the acquisition and interpretation of TEM images is outside the scope of this dissertation. Here I will provide a simplified introduction to the topic and how the information it provides will be used to characterize the nanoparticle deposition system. An excellent starting resource to learn more about all aspects of the transmission electron microscope can be found in the book Transmission Electron Microscopy: A Textbook for Materials Science by D.B Williams and C.B. Carter [58].

\section{Abbe Diffraction Limit and de Broglie Wavelength}

The core concept behind electron microscopy comes from the theory of the diffraction limit proposed by Ernest Abbe in 1873:

$$
d=\frac{\lambda}{2(n \sin \theta)} \approx \frac{\lambda}{2}
$$

Where $d$ is the resolvable feature size, $\lambda$ is the wavelength and $n \sin \theta$ is known as the numerical aperture, with $n$ being the refractive index and $\theta$ the half angle of the lens. This states that the ability to resolve two objects in a microscope is necessarily limited to about half the wavelength of the light being used to image. $550 \mathrm{~nm}$ green light is approximately the middle of the visible range which makes the resolution limit for a conventional light microscope between 250 to $300 \mathrm{~nm}$. This fundamental limitation makes it impossible to use normal visible light to image extremely small structures such as nanoparticles.

Extending the range of microscopy seemed a lost cause until 1924 when de Broglie introduced the concept of wave-particle duality [5]. De Broglie realized that at high energies, particles behave like waves. From de Broglie's theory, the wavelength of an electron (neglecting relativistic effects) is:

$$
\lambda=\frac{h}{\left(2 m_{0} e V\right)^{1 / 2}}
$$

For a $100 \mathrm{keV}$ electron, the wavelength is around $0.004 \mathrm{~nm}$. If used to produce images in a microscope, this would be well below the size of an atom. Above $100 \mathrm{keV}$, relativistic effects become more important and should be included. Also note that the wavelength decreases as the energy of the electron increases. The wave nature of the electron allows them to be used in a microscope to surpass the visible light diffraction limit and image objects well below the limits of conventional light microscopes. In practice, the TEM does not have a resolution as high as the wavelength would allow. This is because the electromagnetic optics used to focus and control the high energy electron beam are imperfect and induce resolution limiting aberrations. However, the resolution can still suffice to image atomic lattices in high resolution TEM instruments. 


\section{Chapter 8 Fundamentals}

\section{Typical Transmission Electron Microscope}

Figure 8.6 shows a simplified diagram of the components of a transmission electron microscope. Real TEMs may have additional lenses, a number of beam limiting apertures, stigmators, deflection coils to translate the electron beam, and more - however, these are not necessary to understand the basic functionality of the microscope. The angles in this figure are extremely exaggerated for illustrative purposes and most angles concerning electron trajectory in a real microscope are less than $1^{\circ}$.

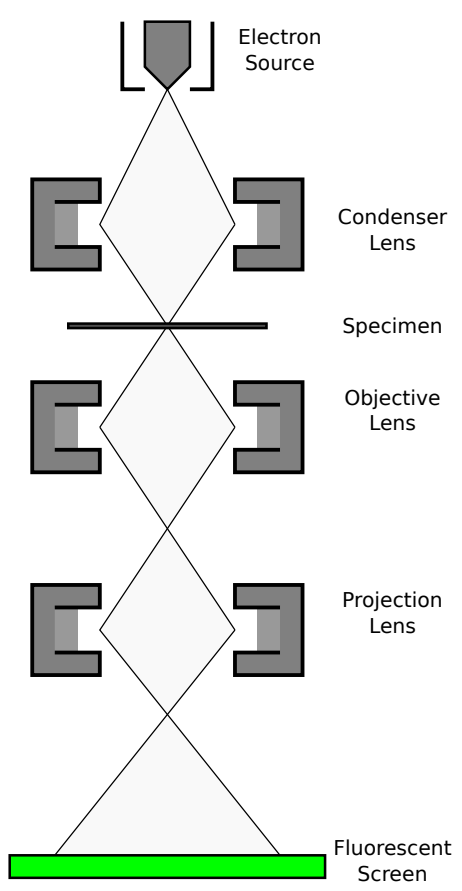

Figure 8.6: Drawing of the typical components in a transmission electron microscope. A beam of high energy electrons is formed in the source and focused through a thin specimen by electron optics. The image is projected by additional optics onto a fluorescent screen for viewing.

A brief overview of the components which make up the transmission electron microscope shown in the figure:

- At the top of the TEM column is the electron source. There are two types of electron sources - thermionic and field emission. Each has its own advantages and disadvantages, though detailed discussion is outside the scope of this description. The electron beam is accelerated to high energies, typically 100 or $200 \mathrm{keV}$, though it can be $1 \mathrm{MeV}$ or more.

- The high energy electron beam generated in the source is focused and columnated through at least one electromagnetic condenser lens onto the sample specimen. The sample must be extremely thin $(<50 \mathrm{~nm})$ to allow the electron beam to pass through 
it. This is one of the larger limitations for performing TEM since not every substrate can be used.

- Another set of electromagnetic lenses are located after the specimen. The first lens is called the objective lens and the second is called the projection lens. These are used to produce the image of the sample and magnify it onto the viewing screen.

- The magnified image is typically focused onto a fluorescent screen where the user can view the image. For permanent recording, the image can be focused onto conventional photographic film, or more recently, CCD cameras for digital recording.

As the electron beam is disturbed by collisions with atoms, the entire column must be kept under high vacuum conditions to produce high quality images. This is another limitation of traditional TEM as it does not allow for viewing objects such as catalytic materials under reactive conditions.

\section{Bright-Field Imaging and Diffraction}

There are several contrast forming mechanisms in the TEM related to electron absorption and diffraction and correspondingly different operating modes to produce images. The most common method for imaging is called Bright Field (BF) imaging. In this method, the direct, un-scattered electron beam is imaged by blocking the diffracted portions of the electron beam with an aperture. Most electrons passing through the specimen are unaffected and pass straight through. This makes the direct beam the brightest spot which gives it the name bright field imaging. The image contrast is produced by the loss of electrons due to scattering. There is greater loss in thicker portions of the sample or in areas with larger atomic number atoms and therefore these regions will appear darker in the image. Alternatively, the diffracted portion of the electron beam can be selected by an aperture and the direct beam blocked. Images produced in this way are called Dark Field (DF) images. The images obtained in this dissertation are all done in a bright field imaging mode.

Instead of producing an image of the specimen, the diffracted beams can be focused onto the imaging plane to produce diffraction images. Spots in the diffraction image will have spacing corresponding to periodic lattice spacings in the specimen. The diffraction images can be very useful for identifying materials based on the lattice spacings.

\section{Additional Techniques}

Due to the interaction from high energy electrons, materials in the sample can produce Xrays, which allows for several additional analytical techniques to be performed in a TEM with some additional detector equipment. These mainly include Energy Dispersive XRay Spectroscopy (EDX or EDXS) and Electron Energy Loss Spectroscopy (EELS). EDX spectra were obtained in the course of this dissertation work and more detailed information regarding this technique is provided with the data in Chapter 10. 
Chapter 8 Fundamentals 


\section{Chapter 9}

\section{Experimental Setup and Instrumentation}

This chapter covers the design and construction details for each instrument of the size selecting nanoparticle deposition system. This includes model number of commercially available parts, design details for home-built components, information on power supplies and electronics, and important information regarding the procedures used to deposit nanoparticles on surfaces. First, an overview of the entire instrument and its configurations is presented. Then each chamber will be individually discussed in detail, moving from the source where clusters are generated, to the mass filter which performs the size-selection, to the surface chamber where the clusters are finally deposited. Additional details will be given for a home-built time of flight mass analyzer used to characterize the performance of the system.

\subsection{Instrument Overview}

An overview schematic diagram of the fully-assembled instrument can be seen in Figure 9.1. The instrument went through a number of intermediate configurations, and any differences from this diagram when some characterization data was obtained will be described when necessary. The final configuration consists of, listed from left-to-right in Figure 9.1:

- The magnetron sputtering source where metal nanoparticles are generated with a wide size distribution. A significant fraction of the generated particles are ionized.

- A quadrupole mass filter which is used to size select the ionic portion of the nanoparticles exiting the magnetron source.

- A set of ion optics which includes deflection plates to correct the ion trajectory leaving the quadrupole and an einzel lens to focus the ions into a better controlled beam.

- The surface deposition chamber which features a second set of ion optics, a substrate holder, and a load-lock system. The load-lock allows for samples to be removed and inserted without the need to vent the UHV surface chamber. 


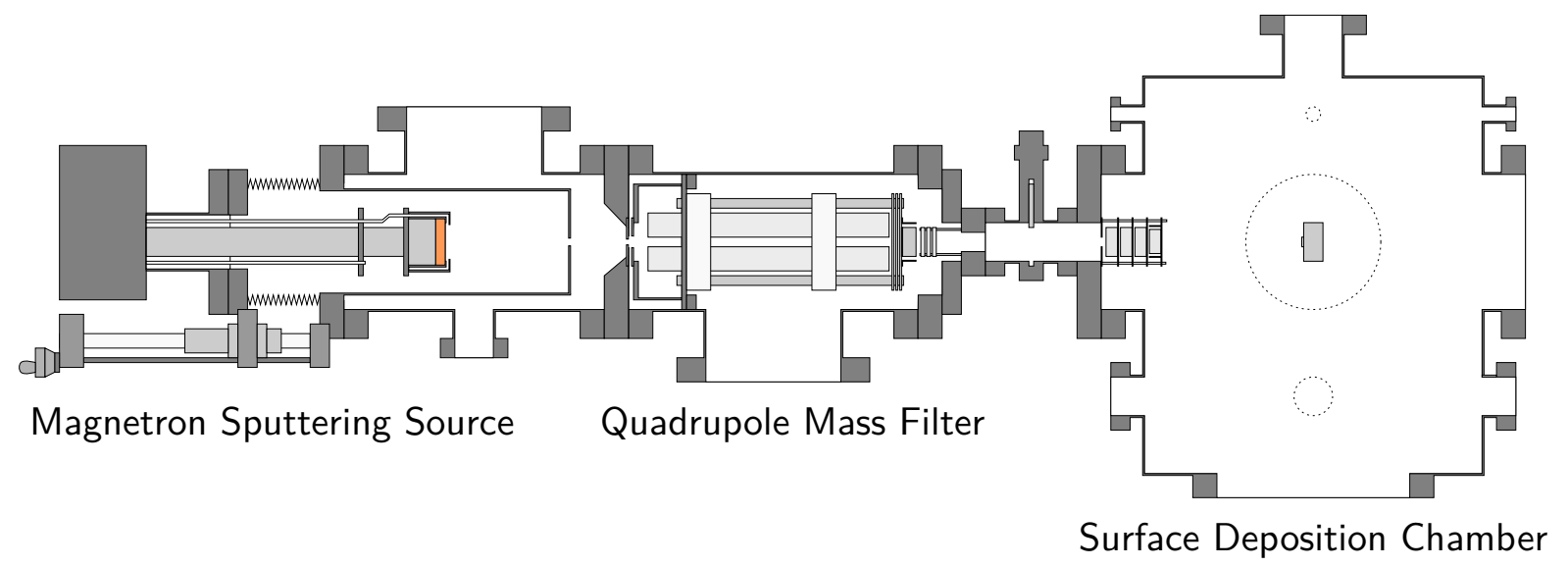

Figure 9.1: Schematic diagram overview of the fully assembled size selected deposition instrument. The components from left to right include: magnetron sputtering ion source, quadrupole mass filter, first ion optics, gate valve, second ion optics, substrate.

Each of the chambers and their components will be discussed in more detail in the subsections below.

For characterization of the source and filter, a temporary second configuration was used, inserting a home-built time of flight mass analyzer in place of the surface deposition chamber. This configuration can be seen in the diagram in Figure 9.2. The time of flight chamber was designed to fit in-between the quadrupole and surface chamber and be available at all times for analysis purposes. However, the transmission of the ion beam over long distances is poor and leaving the time of flight chamber in place reduces the ion current in the surface chamber to levels not viable for deposition. If the ion optic system can be improved, it could be possible to make this analyzer a permanent part of the instrument.

\subsection{Magnetron Sputtering Nanoparticle Source}

The NC200U-B magnetron sputtering nanoparticle source is a commercially available instrument purchased from the company Oxford Applied Research Ltd. located in the United Kingdom. Its design is based on the magnetron instrument developed by Professor Chris Binns [2]. The chamber and components are made with UHV compatible materials. A detailed schematic diagram of the important components of the source chamber can be seen in Figure 9.3.

The magnetron chamber consists of a high pressure gas aggregation region containing the magnetron sputtering head and a differentially pumped outer region to quickly reduce the pressure by several orders of magnitude. The chamber is fitted with an 1,100 L/s magnetically levitated compound turbomolecular pump (Osaka Vacuum model TG1113EM). Pressure inside the aggregation region is monitored during source operation by a Pirani 


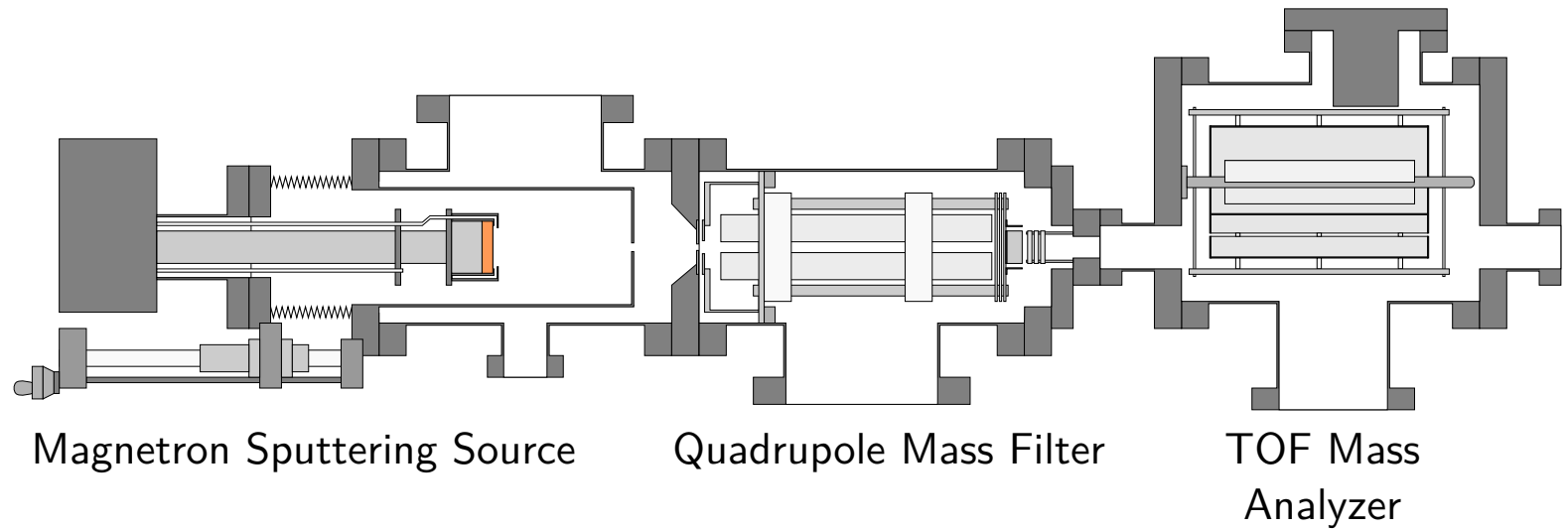

Figure 9.2: Schematic diagram overview of the time of flight analyzer configuration of the instrument. The time of flight analyzer chamber fits in-between the quadrupole and surface deposition chambers. The components from left to right include: magnetron sputtering ion source, quadrupole mass filter, ion optics, home-built time of flight mass analyzer.

gauge, with pressures typically in the 0.09 to 0.5 torr range. The pressure in the outer chamber varies by several orders of magnitude when the source gas is on. A "combi-gauge" (Pfeiffer Vacuum) is used to monitor the pressure in this region. This gauge is a combined Bayard-Alpert and Pirani gauge which automatically switches between the sensors based on the chamber pressure. This allows the pressure to be continuously monitored from pressures when the source gas is on of around $10^{-3}$ torr to $10^{-8}$ torr when the source gas is off.

The sputtering source consists of a 2 inch diameter metal target secured in front of the magnetron head. A thin molybdenum plate is placed between target and magnetron head to protect the head from sputtering. A grounded outer shield (the anode) is spaced at a gap of $0.3 \mathrm{~mm}$ from the target (the cathode). The sputtering gas (typically argon) is introduced in between this gap by a gas line. A DC power supply (Glassman Europe Ltd. LP-600-2) strikes a discharge between the anode and cathode to ignite the sputtering gas into a plasma.

A second gas inlet, used for the aggregation or cooling gas, opens into the aggregation region itself, not directly in front of the sputtering target. This optional gas is typically helium, and it aids the growth of clusters by removing excess internal energy. A third gas feedthrough in this region is connected to the aforementioned Pirani gauge which monitors the pressure. The gas flow into the aggregation region is controlled by two mass flow controllers (MKS 1179B).

The magnetron head and sputtering target are water cooled to prevent excessive heating during operation. The temperature of the target during operation has not been measured, however, in one experiment, indium (melting point of $156.6^{\circ} \mathrm{C}$ ) was used as a sputtering target and quickly melted. Low melting point metals are not advised in this set up. The same cooling water line is used to cool the walls of the aggregation region. Alternatively, the 


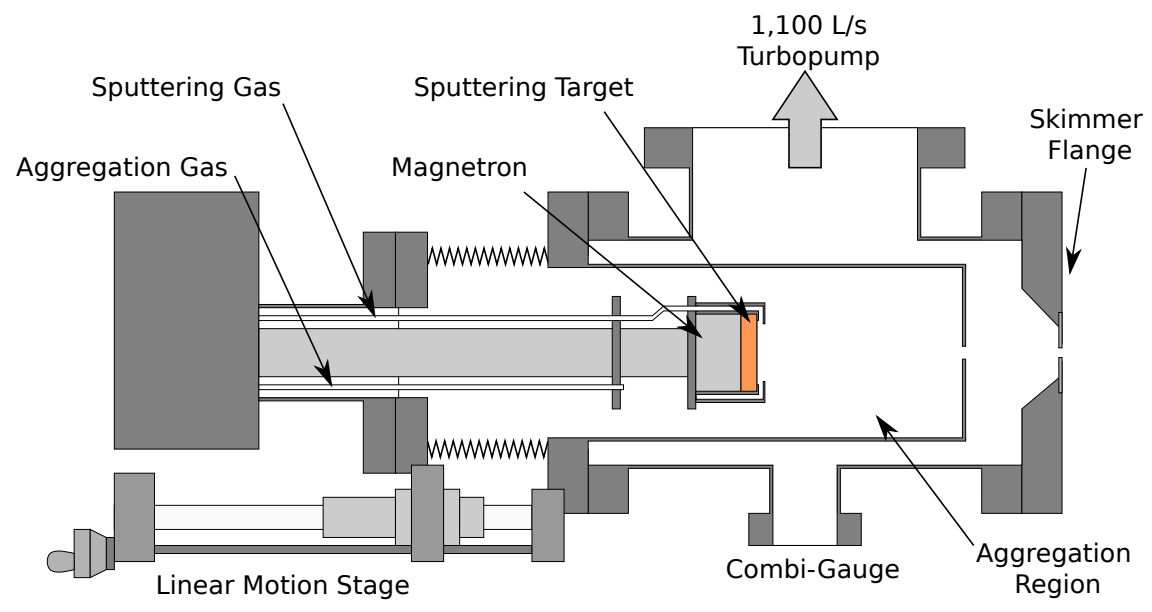

Figure 9.3: Schematic drawing of the magnetron sputtering source chamber with key components labeled.

aggregation region is specified to be able to be cooled down to liquid nitrogen temperatures. This has not yet been attempted in this instrument.

The entire magnetron sputtering assembly is mounted to a linear motion stage which can be used to adjust the distance from the sputtering target to the exit aperture of the aggregation region. This allows for the length of the aggregation region to be varied while in operation. The linear motion drive has a maximum range of motion of $100 \mathrm{~mm}$. The minimum length of the aggregation region is $65 \mathrm{~mm}$ from target to aperture.

A two-sided DN160 CF flange, referred to as the "skimmer flange" separates the magnetron chamber from the quadrupole chamber. This flange has a small aperture in it to regulate the differential pumping between chambers and improve the pressure in the quadrupole chamber. Note that this is not a true skimmer as referred to in molecular beam science. The apertures at the exit of the aggregation region and on the skimmer flange can be replaced with several different sizes, but the system must be vented to change the size. The typically used apertures are $3 \mathrm{~mm}$ for the aggregation region and $5 \mathrm{~mm}$ for the skimmer flange. A design which allows for a variable aggregation region aperture that can be adjusted while the source is in operation is a highly recommended future modification to this set up.

\subsection{Quadrupole Mass Filter}

The QMF200 quadrupole mass filter is also a commercially purchased instrument from Oxford Applied Research Ltd. It is based on the wide mass range quadrupole design of Professor Chris Binns at the University of Leicester discussed in the previous chapter. This quadrupole is specifically designed to work with the mass range of nanoparticles produced in the NC200U-B magnetron sputtering source. 
In order to accommodate the high masses with wide ranges which are produced in the cluster source, this quadrupole has an unusual design compared to normal quadrupole instruments. First, the size of the rods used in this design is large $-25 \mathrm{~mm}$ in diameter. Second, the RF frequency is very low - in the $\mathrm{kHz}$ range. Typical quadrupoles operate with $\mathrm{MHz}$ frequencies. The combination of the large rods and low frequencies theoretically allows for masses between 30 and $3 \times 10^{6}$ amu to be selected.

A schematic diagram showing the key components of the quadrupole filter chamber is shown in Figure 9.4. Apart from the quadrupole itself and the ceramics used to position and support the rods, there are several electrodes near the exit, with added home-built ion optics to allow better control over the ion beam.

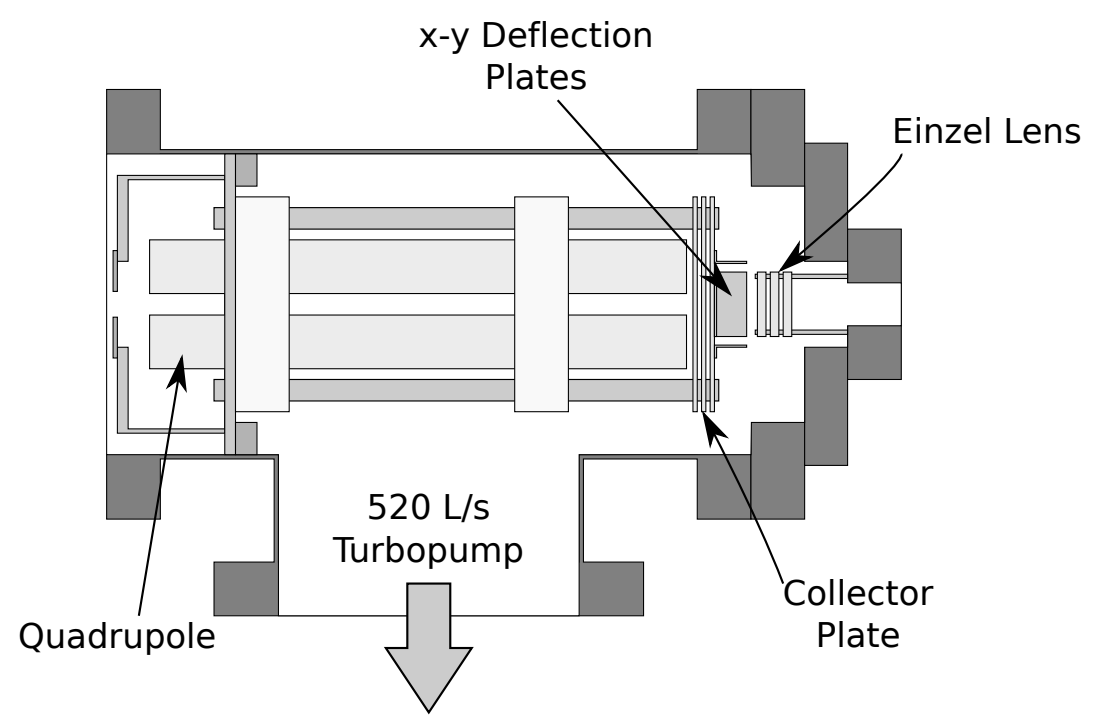

Figure 9.4: Schematic drawing of quadrupole mass filter chamber with key components labeled.

The quadrupole is controlled by a power supply built by Oxford Applied Research Ltd. for this instrument. It can supply up to $250 \mathrm{~V}$ of RF voltage, up to $50 \mathrm{~V}$ of DC voltage, and frequencies between 3 and $100 \mathrm{kHz}$. The DC voltage component is not directly adjustable, but instead is set by a ratio of DC $(U)$ to $\mathrm{RF}(V)$ voltage referred to as "Resolution" in the controller. The resolution control varies between $100 \%$ and $2 \%$ corresponding to $0.042 \mathrm{U} / \mathrm{V}$ to $0.165 \mathrm{U} / \mathrm{V}$. Details regarding how the ratio of these voltages affect the resolution of a quadrupole can be found in Chapter 8 in the section on quadrupole theory. The controller displays the selected transmission mass calculated from the equation previously shown in Chapter 8:

$$
M=7 \times 10^{7}\left(\frac{k V}{f^{2} d^{2}}\right)
$$

where $k$ is a correction factor determined though empirical observation and can be set to any value between 0.5 and 2 in the the control electronics (default setting is 1 ). In an 
ideal situation, $k$ would not be needed, however imperfections in the mechanical design and losses in the RF voltage over long cables can lead to deviations from the optimal calculation which this $k$ value then accounts for. More on the role of $k$ and its determination for this instrument can be found in the characterization of the quadrupole in Chapter 10.3.

There is a series of three thin metal plates at the exit of the quadrupole with small apertures in the center. The outer two plates are grounded and have larger openings (12 $\mathrm{mm}$ for the first plate and $6 \mathrm{~mm}$ for the third plate), while the center plate has a smaller $4 \mathrm{~mm}$ aperture. Due to the smaller opening, some portion of the filtered ion beam collects on this plate. The current generated by these ions can be read on an external picoammeter (Keithley) and this ion current serves as an indication of how many ions of a given filtered mass are present in the beam. This ion current measurement is useful for tuning the source conditions to maximize ion current at the desired mass.

On the third plate, around the exit aperture, four additional small plates which face the ion beam are mounted and a small bias voltage can be applied to two of the plates (the other two are grounded). This allows for steering the ion beam in the $x$ and $y$ directions as it exits the quadrupole. In general, the ion beam is well aligned and this steering voltage is not necessary for deposition.

The additional ion optics mounted after the quadrupole consists of an einzel lens array mounted on a two-sided DN40 CF flange. Applying a DC voltage to the center electrode of this lens greatly increases the ion current which can be collected in the surface deposition chamber and is essential for deposition. The DC power supply (TTi PLH250-P) can provide up to $250 \mathrm{~V}$ at $0.375 \mathrm{~A}$. However, this is a crude method for improving the extraction of ions from the quadrupole. Modifications to improve the ion optics and better guide the ions from the quadrupole exit to the substrate surface should be done in the future to maximize the performance of the instrument. Up to now, this einzel lens functions well enough to provide a sufficient number of ions for deposition purposes.

The quadrupole chamber is pumped by a $520 \mathrm{~L} / \mathrm{s}$ turbomolecular pump (Pfeiffer Vacuum TMU 520). The pressure inside the chamber is typically in the low $10^{-8}$ torr range when the cluster source is not running and low $10^{-5}$ torr when the source is on.

\subsection{Time of Flight Mass Spectrometer}

\section{Instrument Design}

The size selected nanoparticle deposition system is a completely new instrument being built, and therefore it is desirable to be capable of independently characterizing its performance. For this purpose, an additional instrument was designed and custom built in the mechanical workshop at UCSB to perform time of flight mass spectrometry on the mass filtered cluster ion beam and determine the performance of the quadrupole filter. Many time of flight analyzers are very large with field-free flight distances of over a meter to improve the resolution. A more compact design was desired for this application, to fit into the existing instrumental footprint. Having a high, better than 1 amu resolution is 
not necessary for this application, as the nanoparticle mass distribution will already be quite broad. Even under the optimal filtering conditions, $2 \%$ is the best resolution to be expected. This makes a short flight path instrument acceptable to the application.

The electrode geometry of the mass spectrometer is based on the lateral time of flight mass filter designed by B. von Issendorf and R.E Palmer [1]. Instead of applying a symmetric stopping pulse to the ions to filter them, the pulsed ions exit through a mesh covered opening in the top of the filter and are collected on a detector. A schematic drawing of the final home-built time of flight mass analyzer chamber can be seen in Figure 9.5.

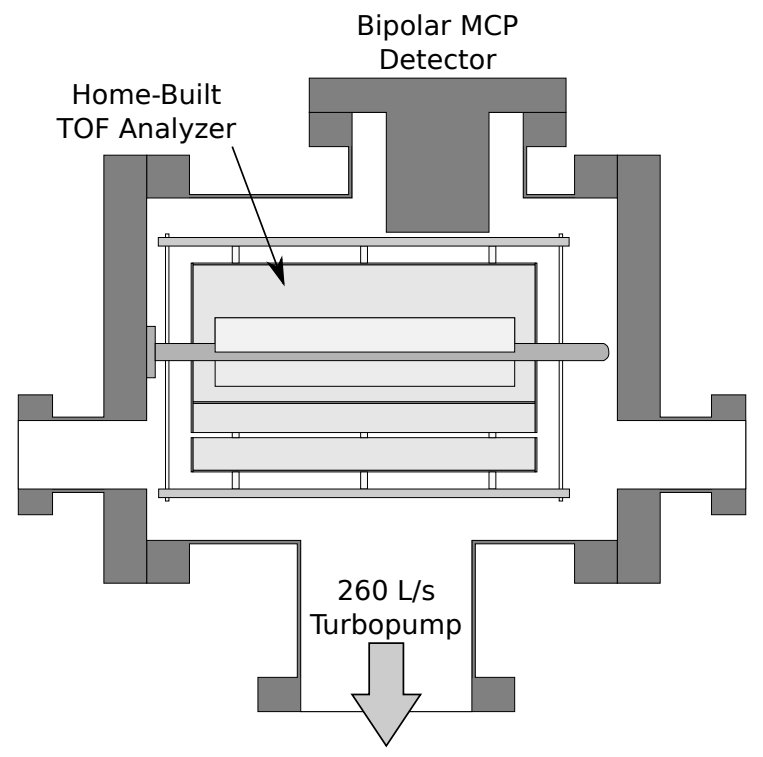

Figure 9.5: Schematic drawing of time of flight mass analyzer chamber with key components labeled.

A cross-section of the time of flight analyzer itself can be seen in Figure 9.6. A potential is applied to the pulse plate creating an electric field in the region between the bottom plate and the central plate, which is at ground potential. This gives the ion beam an acceleration in the lateral direction. Once the ions pass the mesh in the center plate, they are in a field free region. At this point their velocity is fixed from the energy they received from the accelerating pulse. Ions with higher masses have slower velocities compared to those with lower masses. Once the ions pass the mesh at the exit of the field free region, they are then accelerated to the detector which is biased to $8 \mathrm{kV}$.

\section{Simulations}

Before building, the instrument was simulated in SIMION [8] to test its theoretical performance and ensure it meets the desired requirements. SIMION is a professional software package which can simulate ion trajectories in electric fields. By modeling the electrode 


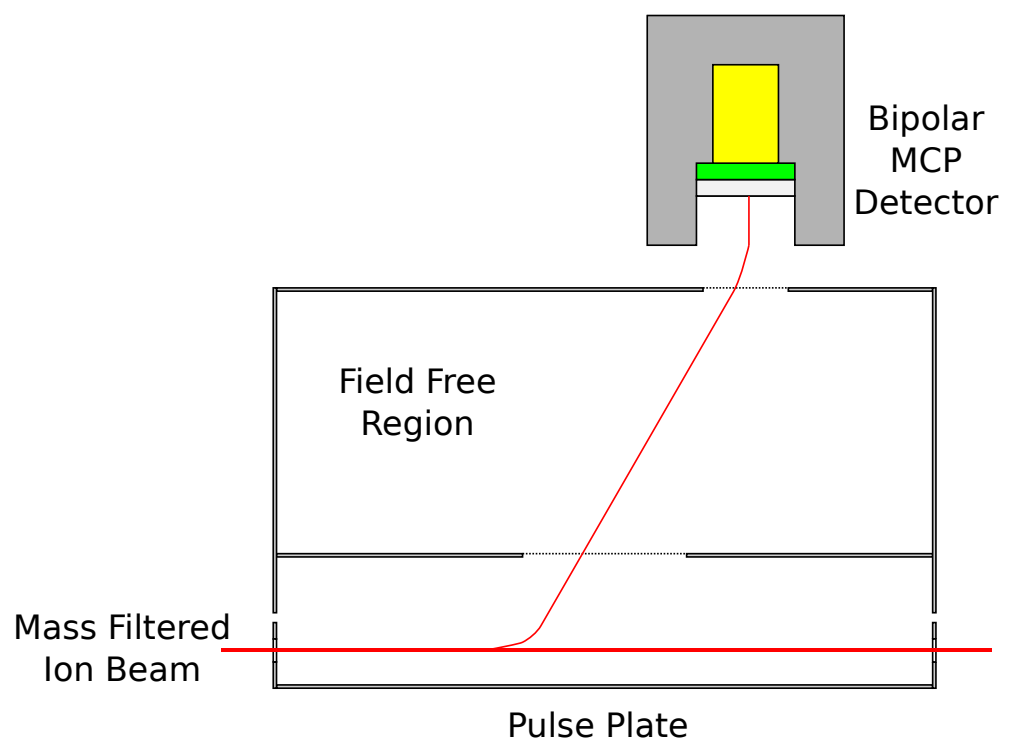

Figure 9.6: Drawing illustrating the operation of the home built time of flight mass analyzer. A high voltage pulse applied to the bottom plate deflects the ion beam through a $90 \%$ transmission mesh into the field free region. The ions exit the field free region through a second mesh and are accelerated to $8 \mathrm{keV}$ into the MCP detector. The electrons produced by the MCP are converted into photons by a scintillator (green) and then back into an electrical signal by a photomultiplier tube (yellow).

configuration of the analyzer, simulated times of flights can be generated quickly and easily for ions of any reasonable mass, position, energy, etc.

Here I will show some results of the simulation related to the functionality of the instrument. The geometry and programming files used for these simulations can be found in the Appendix. I will refer back to the simulation in direct comparison to experimental results in the characterization of the built instrument in Chapter 10.2.

Figure 9.7 shows an example set of trajectories calculated from the SIMION simulation of the time of flight analyzer. The ion beam in this simulation is for 50,000 amu clusters with a forward kinetic energy of $15 \mathrm{eV}$. The high voltage pulse is $1000 \mathrm{~V}$ for 200 usec. Although only a small fraction of the overall ions reach the detector, the geometry was designed to accommodate a wide range of potential situations which may occur. The angle of the trajectory of the ions is determined by a ratio of the forward kinetic energy to the kinetic energy gained in the pulse. If there is a mixture of ions with a range of forward kinetic energies, then the portion of the pulse region they start from in order to reach the detector will vary. The wide opening in the field free region allows more possible angles to reach the detector in the same pulse.

To determine a theoretical limit on the mass resolution of the analyzer, the arrival times 


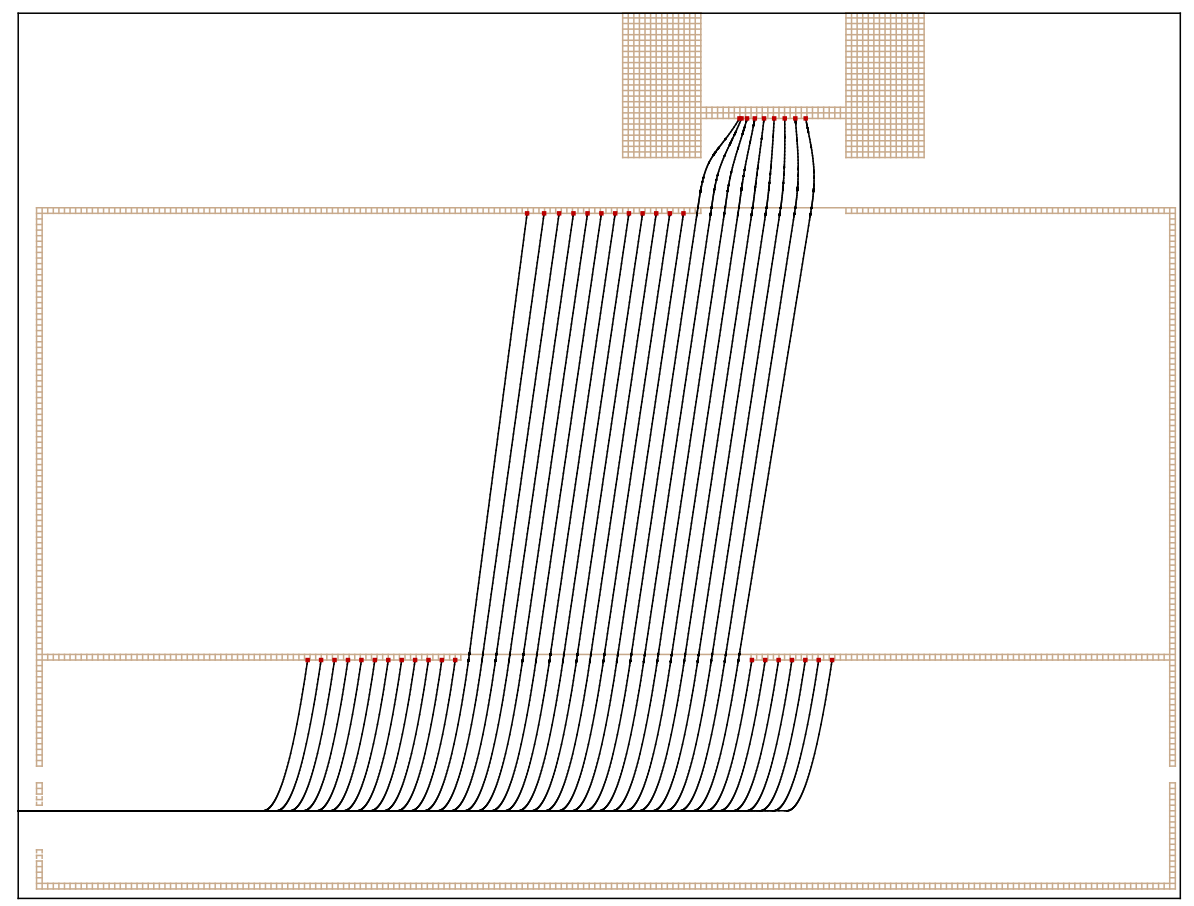

Figure 9.7: Screenshot of an ion trajectory simulation from SIMION illustrating the operation of the home built time of flight mass analyzer.

of a given mass were converted into a Gaussian by using the mean detector arrival time as the location of the Gaussian peak and the standard deviation of the arrival times as the FWHM of the Gaussian. The result for the 50,000 amu simulation is indicated as a black curve in Figure 9.8. The process was repeated to find the smallest increase in mass which fits the $50 \%$ valley definition in mass spectrometry for two peaks being resolved. 51,500 amu (the red curve in figure 9.8) was found to be just about at the limit for being resolved under this definition. This sets the theoretical resolving power from the simulations at 33.3 or $3 \%$ of the peak mass. While this is low by conventional time of flight mass spectrometry standards (which can have resolving powers $>5000$ ), it is acceptable given the maximum $2 \%$ mass resolution of the quadrupole filter. It should be noted that these simulations were done for optimal conditions of the ion beam (narrow, no divergence). As with the quadrupole, in reality, the analyzer can be expected to have a lower performance than this.

More results from this SIMION simulation will be presented in Chapter 10 with a comparison of actual experimental performance and flight times to the simulated flight times.

\section{Construction Details}

A few details regarding the construction of this instrument will be described here, as it was constructed entirely in-house by the mechanical workshop of the Department of Chemistry and Biochemistry at the University of California, Santa Barbara.

The thin plates making up the top, middle, bottom, front and back of the time of 


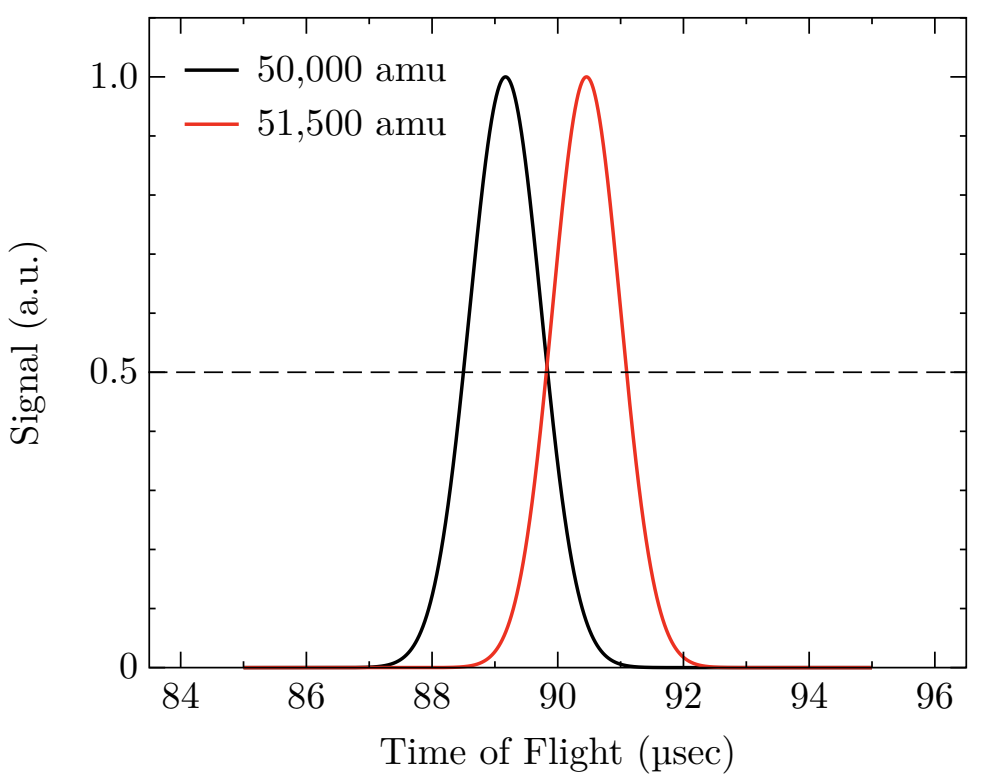

Figure 9.8: Simulated TOF peaks generated by converting the SIMION calculated TOF parameters into a Gaussian function showing the calculated resolving power of the instrument with a $50 \%$ valley definition.

flight analyzer are made from $1 \mathrm{~mm}$ stainless steel. The side walls which provide the structural support are made from $5 \mathrm{~mm}$ thick aluminum. There are three solid $\mathrm{Al}_{2} \mathrm{O}_{3}$ ceramic supports which run through the entire instrument on each side. Spacers of the same ceramic surround these supports and create the gap between bottom electrode and top grounded field-free region. Three more spacers at the top and bottom between the time of flight instrument and an aluminum support bar provide pressure which holds the instrument together. The entrance and exit plates have $7 \mathrm{~mm}$ apertures. An electrically isolated metal plate could be mounted behind the exit aperture to allow measurement of the ion current when needed. The space between the extraction plate and the grounded plate which is the entrance of the field free region is $40 \mathrm{~mm}$. The ion beam enters this region $11 \mathrm{~mm}$ from the bottom extraction plate. The field free flight distance is $80 \mathrm{~mm}$.

Fine nickel mesh with $90 \%$ transmission is spot welded to support frames which are secured over the openings of the plates surrounding the field free region. This allows the frames to be detached if the mesh becomes damaged and easily replaced. The center plate has a $50 \mathrm{~mm}$ by $12 \mathrm{~mm}$ rectangular opening and the top plate has a $25 \mathrm{~mm}$ diameter circular opening (to match the $25 \mathrm{~mm}$ sized MCP detector).

Electrical connections are made by securing a vacuum compatible Kapton coated wire beneath a washer on one of the screws which holds the side plates together. The wire is connected to a BNC electrical feedthrough welded into one of the D200 CF end cap flanges on the chamber by a $\mathrm{BeCu}$ connector.

The instrument is secured in the chamber by two "L" shaped pieces of aluminum bolted to the outer side walls of the instrument which allow it to "hang" on support rods which 
are mounted to one of the DN200 CF end cap flanges.

The chamber is constructed from stainless steel tube. The ends have DN200 CF flanges welded on. Two additional tubes with DN100 CF flanges are welded on to allow for pumping and for connecting the detector precisely in position above the analyzer. The chamber is pumped by a $260 \mathrm{~L} / \mathrm{s}$ turbomolecular pump (Pfeiffer Vacuum HiPace300). The pressure in this chamber is typically in the low $10^{-7}$ torr range.

\section{Detector}

The detector used for this time of flight analyzer is a $25 \mathrm{~mm}$ Bipolar Time of Flight MCP Detector from Photonis. This detector consists of a series of $25 \mathrm{~mm} \mathrm{MCP}$, in front of a phosphor screen scintillator, in front of a photomultiplier. In the case of negative ions, the ions are accelerated to the multichannel plate which is biased to $+8 \mathrm{kV}$. Impact with the MCP produces a cascade of electrons which are ultimately emitted from the back of the plate when a bias voltage is applied across the MCP, typically around $800 \mathrm{~V}$. Therefore the back will be biased to $+8.8 \mathrm{kV}$. These electrons are then converted to light when they hit the phosphor screen scintillator which is further biased $2 \mathrm{kV}$ above the back of the MCP or $+10.8 \mathrm{kV}$ in this example. The light from the phosphor screen is then converted back into electrical current by a photomultiplier and the output current of the photomultiplier can be measured. The advantage to this rather round-a-bout way of measuring current, is that it is very simple to switch from measuring positive ions to negative ions using the same set of electronics. Hence the "Bipolar" naming of the detector. For positive ions, typical voltages analogous to the previous anion example would be $-8 \mathrm{kV}$ MCP front, $-7.2 \mathrm{kV} \mathrm{MCP}$ back, and $-5.2 \mathrm{kV}$ scintillator. Since both positive and negative ions are produced in the cluster source, it is convenient to be able to detect either based upon the desired experimental requirements. In addition, due to the large masses of the analyzed ions, a large accelerating bias voltage is useful to increase the chance of the ion producing a response on the $\mathrm{MCP}$.

\section{Operation}

This section will briefly describe the operation of the instruments used to record a time of flight spectrum.

The DC power supply (Stanford Research Systems PS325) for the extraction pulse can supply up to $2500 \mathrm{~V}$ of either positive or negative polarity. This power supply is connected to a high-voltage pulse generator (DEI PVX-4140). When a $+5 \mathrm{~V}$ trigger voltage is provided from a digital delay generator (Stanford Research Systems DG645), the pulse generator supplies the high-voltage from the DC power supply to the extraction plate for the duration of the trigger pulse from the delay generator.

The response signal of the detector from nanoparticle ions in a single pulse is very low. In order to sum the results of a large number of extraction pulses, a multi-channel scaler (TurboMCS) was used. The MCS is triggered at the same time as the start of the high voltage pulse on the time of flight analyzer. It then parses the signal from the detector 


\section{Chapter 9 Experimental Setup and Instrumentation}

into a series of discrete time bins known as a "dwell" and if the signal output during one bin surpasses a set discriminator level, it records one count in that dwell. It then sums the number of counts in a dwell across the specified number of pulses and outputs the total number of counts recorded for each dwell time interval. This results in a signal which is the sum of a large number of individual pulses. The dwell times used were typically either 5 or 20 nsec and each spectrum was typically obtained with 10,000 extraction pulses. The output of the MCS is collected on a PC using LabVIEW [32] to interface with the instrument.

\subsection{Surface Deposition Chamber}

\section{Specifications}

The last chamber of the instrument is dedicated to all aspects of handling the substrate to be used for nanoparticle deposition. The chamber itself is a UHV compatible, large stainless steel cylinder (35 cm in diameter), with various sizes of ConFlat flanges at three height positions every $45^{\circ}$ around the center axis for a total of 24 accessible CF flanges in addition to one at the top and bottom of the cylinder. The top level is entirely composed of eight DN16 CF ports. The middle level alternates between four DN160 and four DN40 $\mathrm{CF}$ ports. The bottom level alternates between four DN40, two DN63, and two DN100 $\mathrm{CF}$ ports. The majority of these are currently not in use, however they are available for future expansion to make the instrument compatible with a wide variety of possible experiments. A schematic diagram illustrating the essential components currently installed in this chamber is shown in Figure 9.9.

The chamber is pumped by an 1,100 L/s magnetically levitated compound turbomolecular pump similar to the one on the magnetron chamber (Osaka Vacuum TG1110M). The chamber is typically in the $10^{-9}$ torr range without baking. In future experiments where the surface cleanliness is more important, true UHV pressures will be desirable, and should be attainable. The middle level is used for the substrate positioning during deposition and for ion optics. Located between the surface chamber and the quadrupole chamber is a DN40 CF pneumatic gate valve, to allow the surface chamber to stay under vacuum if the magnetron chamber needs to be vented for cleaning or to change targets. The entrance consists of a DN160 to DN40 CF zero length adapter with ion optics mounted facing into the surface chamber. The ion optics include a simple einzel lens array, similar to the one inside the quadrupole chamber, and another set of $x-y$ deflection plates. This einzel lens allows for maximizing the current at the deposition position by refocusing the ions onto the substrate position. The $x-y$ deflection plates are essential for size selected nanoparticle deposition. While the magnetron produces a large fraction of natively ionized clusters, there can still be a large fraction of neutrals which are unaffected by the quadrupole filter. Therefore the mass filtered ion beam is steered off the central axis by the $x$ or $y$ deflection plates and the deposition takes place approximately one inch away from the center beam axis to avoid deposition of unwanted sizes of neutral particles. 


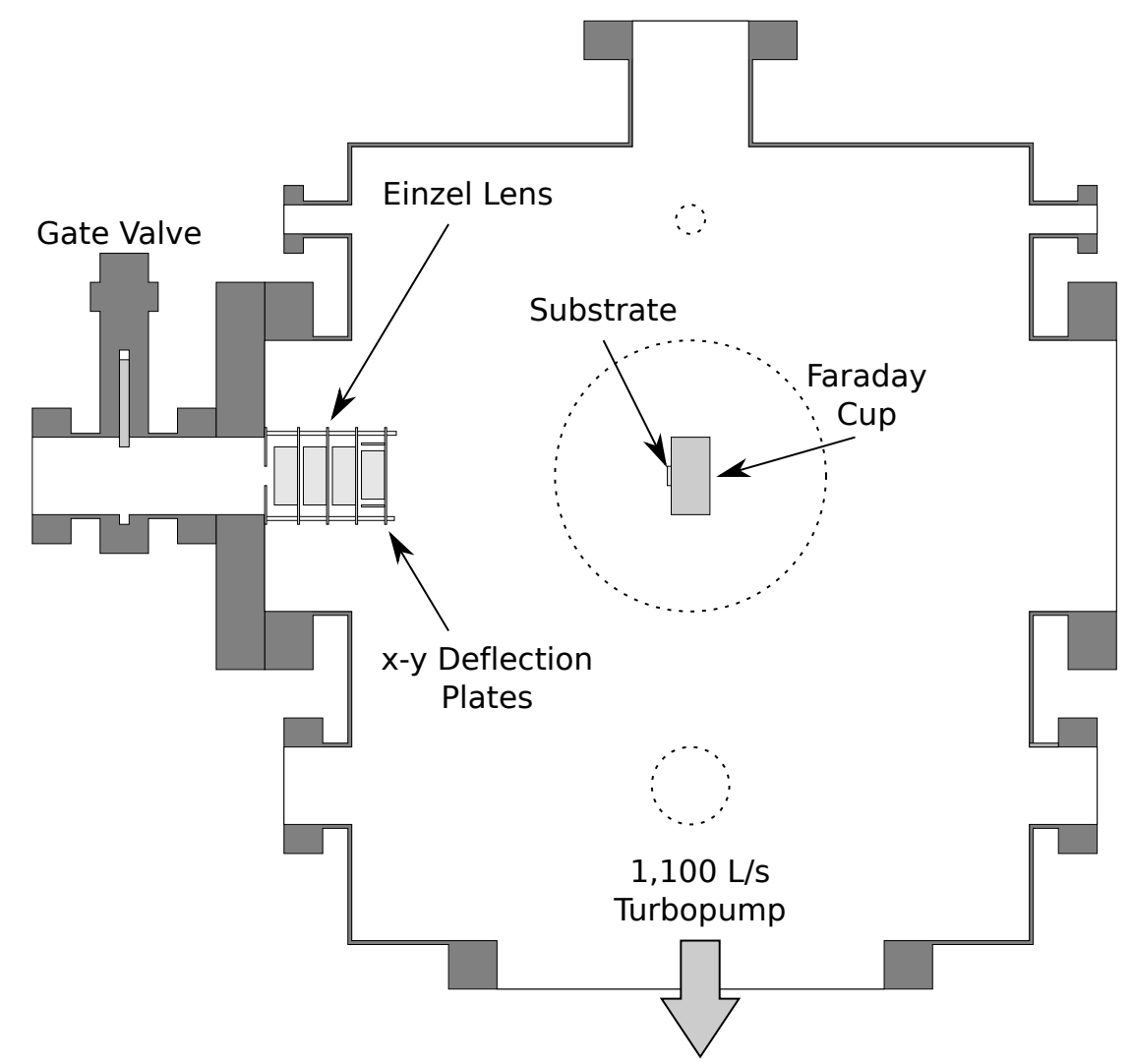

Figure 9.9: Schematic drawing of the surface deposition chamber with key components labeled. 


\section{Chapter 9 Experimental Setup and Instrumentation}

\section{Sample Insertion and Manipulation}

The substrate is inserted into the chamber via a "load-lock" system. A DN100 CF gate valve is installed on one of the flanges at a $90^{\circ}$ angle to the particle ion beam entrance. Behind this gate valve is a DN63 CF cubical chamber with an o-ring sealed door and small $60 \mathrm{~L} / \mathrm{s}$ turbomolecular pump. The substrate is mounted on a commercially available "CabFast" sample holder system from MDC Vacuum. The substrate is securely clamped to a one inch diameter platen sample holder. The sample holder can then be inserted through the door onto the end of a platen fork affixed to the end of a three foot long magnetically coupled, linear motion, rotatable manipulator attached to the cube. After closing and securing the door, the cube can be pumped to $10^{-5}$ torr. Once under vacuum, the gate valve separating the cube from the surface chamber can be opened and the manipulator used to push the substrate from the cube to inside the surface chamber. This system allows the surface chamber to maintain good vacuum while a sample is removed or inserted.

On the back side of the sample holder is a Faraday cup (Kimball Physics). The sample manipulator is rotatable, and the Faraday cup can be positioned so that it measures the ion current directly at the ion beam deposition position on an external picoammeter. Once the ion current at the deposition spot is optimized via the ion optics and deflection plates, the sample holder can be rotated by $180^{\circ}$, exposing the substrate surface to the ion beam for deposition. Photographs of the sample holder with a mounted silicon wafer substrate and the Faraday cup on the reverse side can be seen in Figure 9.10. The measured ion currents used for deposition are typically on the order of $30 \mathrm{pA}$ on a $1 \mathrm{~cm}^{2}$ collector for a $20 \%$ mass resolution ion beam. Deposition is typically done for 10 to 20 minutes. This can be calculated to expect around 1000 nanoparticles per $\mu \mathrm{m}^{2}$ on the surface. The observed microscopy results typically show a density of clusters on the surface on the order of a few hundred nanoparticles per $\mu^{2}$ which is slightly lower than expectation.

\section{Other Components}

Not indicated in Figure 9.9 are some other useful components. A residual gas analyzer (Stanford Research Systems RGA200) can be used to analyze the trace gases in the chamber and as another pressure monitor. An argon ion gun (LK Technologies) is also available to sputter clean the substrate surfaces. This process is not done for these characterization tests. The surfaces prepared in the characterization of the instrument are exposed to atmosphere for microscopy or other analysis, defeating the purpose of achieving a truly clean surface. For future experiments where the sample is kept in vacuum and used, for example, as a surface for molecular beam scattering, the capability is there. 

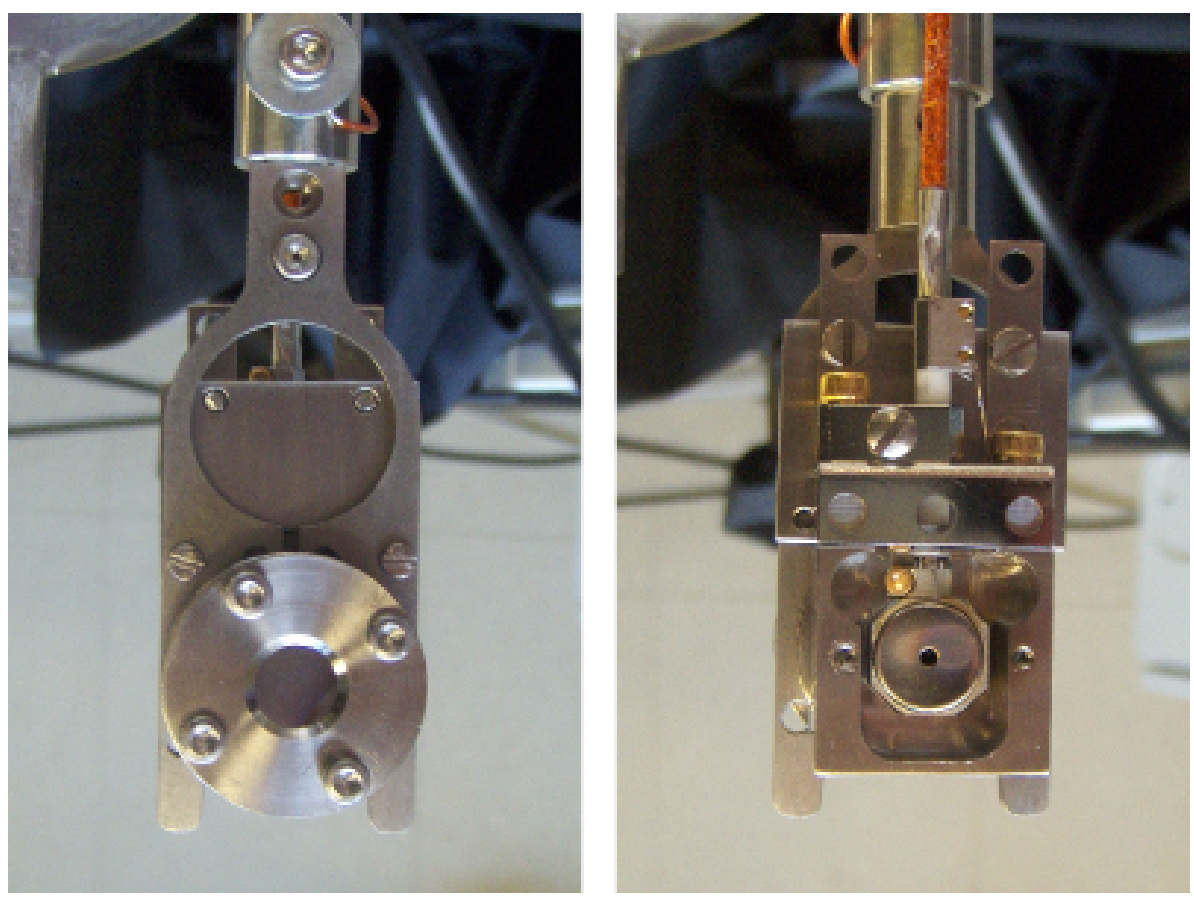

Figure 9.10: Photograph of the sample holder. Left: One side exposes the substrate surface to the ion beam. Right: The reverse side exposes a commercial Faraday cup to the ion beam in a position identical to the exposed substrate for monitoring incoming ion current at the deposition position. 
Chapter 9 Experimental Setup and Instrumentation 


\section{Chapter 10}

\section{Characterization and Preliminary Results}

This chapter covers the characterization performed for all of the instruments which are a part of the complete size selected nanoparticle deposition system. First, the performance of the magnetron sputtering nanoparticle source is examined in terms of its capability to produce nanoparticle ion beams with a wide range of cluster sizes by varying source conditions. The time of flight mass spectrometer is characterized and calibrated to determine the actual mass of nanoparticle ions in the transmitted beam. The system's quadrupole filter is examined in the context of the known masses from time of flight in the transmitted ion beam to determine the accuracy of its performance. The width and kinetic energy of the ion beam is measured prior to deposition. Finally, the ion beam is deposited on substrate surfaces and the characteristics of the deposited clusters examined through microscopy.

\subsection{Magnetron Sputtering Ion Source}

The magnetron sputtering ion source is capable of producing a range of cluster sizes depending on the source conditions. It is critical to understand how the adjustable parameters of the magnetron source influence the overall mass distribution in the initial beam in order to optimize for the maximum intensity at a desired size. Several experiments were performed which focus on how the cluster beam changes as a function of the source conditions used and trends in how these conditions affect the mass distribution are determined.

\subsubsection{Trends in Cluster Size Distribution}

This section will demonstrate the effects the sputtering gas pressure, aggregation gas pressure, aggregation length and sputtering power has on the cluster growth process, with figures showing how the mass distributions evolve when varying each parameter while holding the others constant. For these measurements, a simplified test system was assembled using only the NC200U-B magnetron sputtering source connected to the QMF200 quadrupole mass filter. After the quadrupole exit, a Faraday cup was placed to measure the ion current of the filtered cluster beam. The outer grid of the cup could be biased to $+300 \mathrm{~V}$ to attract negative cluster ions while repelling positive. The anionic portion of the beam was used to rule out any contribution from the argon cations which may also be in the beam. The intention here is more to show the trend each parameter produces on 
the cluster size distribution and not to state with certainty that for a given set of values the same size distribution will result. The masses reported are the masses selected on the quadrupole controller front panel for the default $k$ factor of 1.0. As will be demonstrated later, the actual correction factor is 1.42 and therefore the masses reported in this section are $42 \%$ too low.

\section{Effect of Sputtering Gas Pressure}

First, I will discuss the effect of the sputtering gas pressure. Increasing the argon pressure in the sputtering chamber (by increasing the flow rate of gas into the system) tends to first increase the size of clusters when increasing from low flow rates, and then decreases the peak mass of the distribution when approaching high flow rates.

The effects of increasing argon flow on the cluster mass distribution can be seen in Figure 10.1. The flows are reported in standard cubic centimeters per minute $(\mathrm{sccm})$. The cluster size distributions are measured by the ion current (in nA) on the Faraday cup at the exit of the quadrupole for a particular selected mass. In these magnetron characterization measurements, copper was used as the sputtering target material. The solid lines in all figures are fits of the data points to a log-normal distribution, for reasons which have been previously discussed in Chapter 8. In general, the magnitude of the currents should not be directly compared to each other as the quadrupole transmission is frequency dependent. Lower frequencies are required to reach larger masses and have a smaller transmission window through the quadrupole.

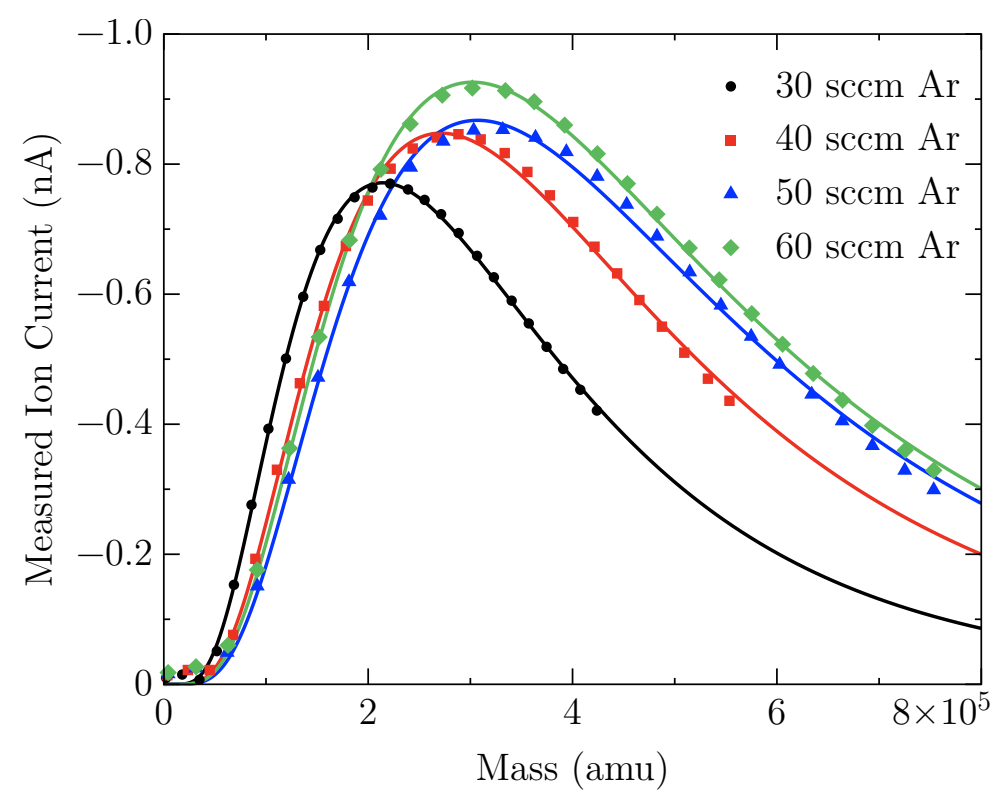

Figure 10.1: Plot of experimental data illustrating the effect of changing sputtering gas (argon) flow on the cluster distribution produced in the magnetron. The points are the experimental data and the matching colored lines are log-normal distribution fits to the data. 
As the flow of Ar increases from $30 \mathrm{sccm}$ to $50 \mathrm{sccm}$, keeping sputtering power and aggregation length constant, the cluster size gradually increases from a peak near 200,000 amu (around 3,000 copper atoms per cluster) to a peak at 300,000 amu (almost 5,000 copper atoms per cluster). Above $50 \mathrm{sccm}$, the peak of the distribution no longer appears to increase. At $60 \mathrm{sccm}$, the maximum of the size distribution appears to shift slightly to smaller sizes, and the peak intensity increases.

In terms of the cluster formation mechanisms discussed in Chapter 8, increasing the argon gas concentration has two effects. First, it increases the amount of metal atoms produced and available for clustering. This should increase the rate of both mechanisms Nucleation and Condensation. Second, more rare gas is available to produce the cluster seeds through the nucleation termolecular collision. Formation of the cluster seed is the rate limiting step in the cluster growth process, considering it requires a termolecular collision.

This produces two competing effects. At first, with the increased availability of sputtered metal atoms, clusters simply grow larger through the condensation mechanism. As the termolecular collision becomes more favorable at high argon pressures, more cluster seeds can be produced. Each seed "consumes" free metal atoms as it grows. If the rate of consumption of free metal atoms by the seed clusters exceeds the rate of production of these atoms, eventually the particle size will reach a limit and begin to decrease.

A hypothetical analogy may illustrate this competition effect better. If there are 1000 free metal atoms available at one pressure and 10 seeds form, if the free atoms are evenly distributed, each fully grown cluster would be made up of around 100 atoms. At a higher pressure, there could be 2000 atoms and 100 seeds. In this case, the clusters would only have 20 atoms in an even distribution.

\section{Effect of Aggregation Distance}

The higher pressure of argon (and helium) in the aggregation region compared to the rest of the vacuum system is required for efficient cluster growth. Once the nanoparticles pass through the exit aperture of the aggregation region into the vacuum system, cluster growth ceases due to the lack of further collisions. This places a physical limitation in the system on how large the clusters are able to grow. The magnetron source is mounted on a linear motion stage which allows the length of the high pressure aggregation region to be adjusted by up to $100 \mathrm{~mm}$. As the stage is withdrawn, the aggregation region becomes longer and the clusters spend more time in the effective growth region before exiting, allowing them to grow larger. In effect, increasing the aggregation region length increases the range of sizes the clusters can grow to. This is demostrated in Figure 10.2.

The minimum aggregation distance is $48 \mathrm{~mm}$. As the aggregation distance increases from the minimum length plus $25 \mathrm{~mm}$ to plus $75 \mathrm{~mm}$, while holding other parameters constant, the peak mass of the cluster distribution increases and the distribution becomes broader. 


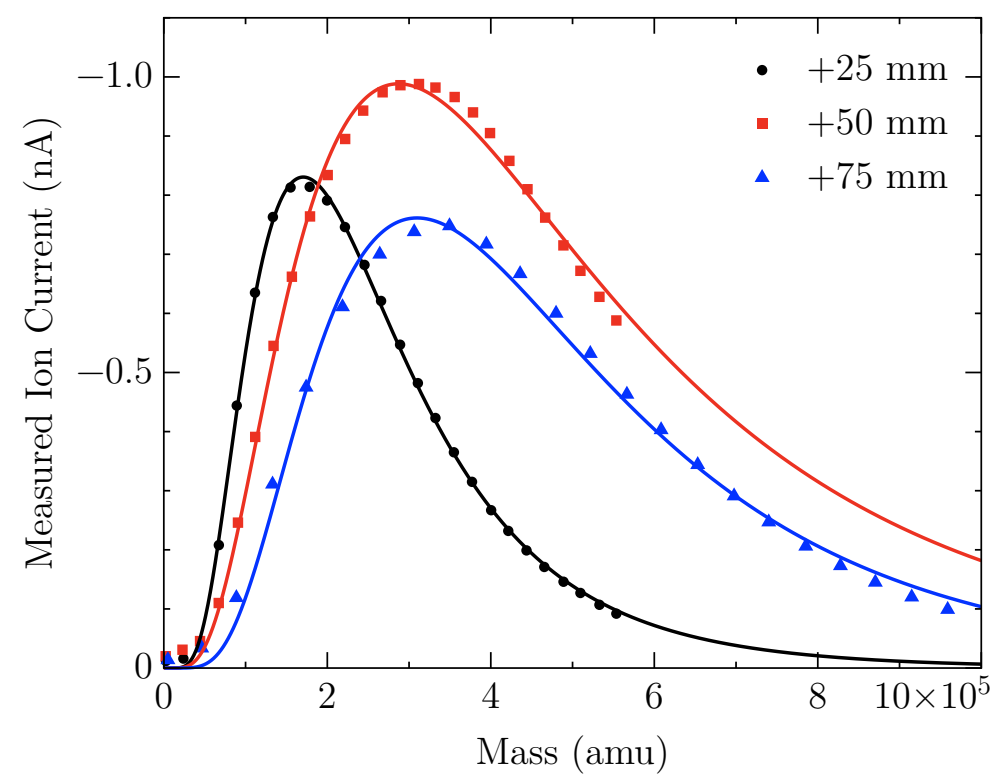

Figure 10.2: Plot of experimental data illustrating the effect of changing the aggregation region distance on the cluster distribution produced in the magnetron. The points are the experimental data and the matching colored lines are log-normal distribution fits to the data. The lengths in the legend are in addition to the minimum distance $(48 \mathrm{~mm})$.

\section{Effect of Magnetron Sputtering Power}

The sputtering power in the magnetron plasma discharge is a function of the voltage and current supplied by the DC power supply. The metal target is negatively biased which drives the positive argon cations in the plasma to the metal surface. The sputtering power is controlled by adjusting the current and voltage through the power supply. As the current through the discharge is increased, more argon cations are formed, increasing the amount of sputtering occurring at the target surface. As the sputtering power increases, more metal atoms are sputtered from the surface, effectively increasing the density of free metal atoms available for clustering. The result is to create larger clusters and higher ion current with higher sputtering power. This can be seen in Figure 10.3.

As the sputtering power increase, the peak intensity and size tend to increase. This is a very useful parameter for controlling the magnetron cluster size distribution as it is easily adjusted and sputtering with a low power creates such a low density of metal atoms that it can effectively limit the size of clusters being formed, even with a long aggregation region or high argon pressure.

\section{Effect of Aggregation Gas Pressure}

The final parameter is the pressure (also controlled by mass flow rate) of a second gas added to the aggregation region, called the aggregation gas. This gas is typically helium. 


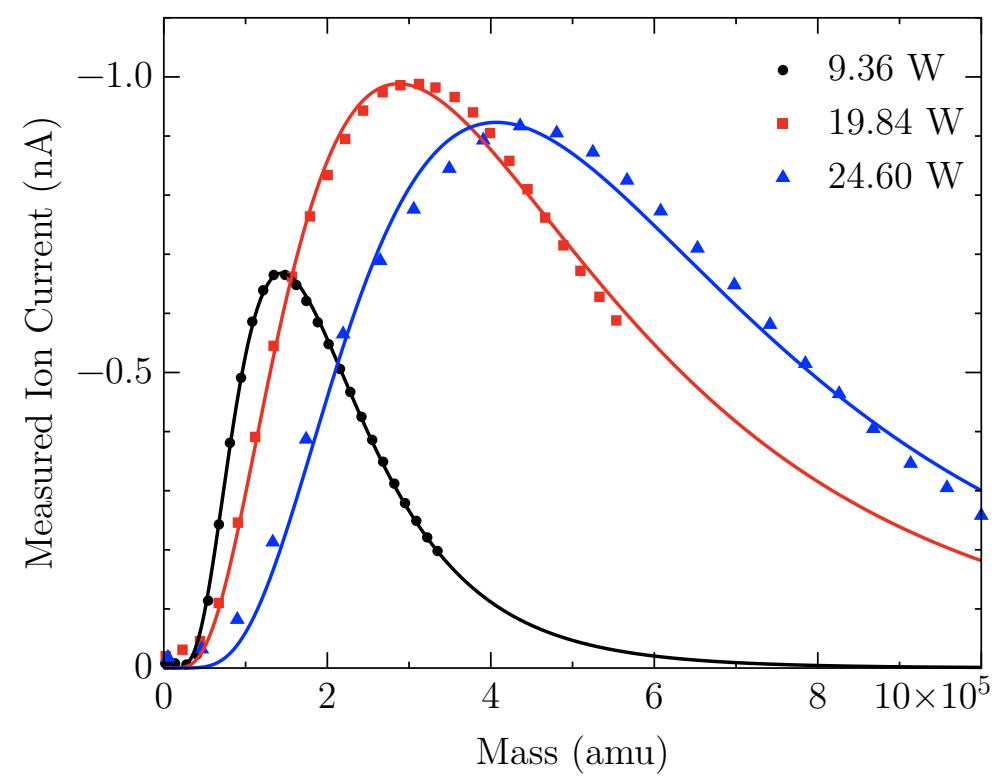

Figure 10.3: Plot of experimental data illustrating the effect of changing sputtering power on the cluster distribution produced in the magnetron. The points are the experimental data and the matching colored lines are log-normal distribution fits to the data.

The effects of increasing helium flow on the cluster mass distribution can be seen in Figure 10.4. As the helium flow increases, the peak cluster mass dramatically decreases. Adding helium to the system does not increase the amount of free metal atoms, as the helium is unlikely to contribute to the sputtering process. It does, however, increase the amount of rare gas available to produce the cluster seed in the nucleation mechanism Nucleation. Similar to the competition effect described in the effect of increased argon flow, with an increase in the amount of cluster seeds, each new cluster consumes free metal atoms as it grows. There are only so many atoms available, therefore the aggregation gas leads to production of many smaller clusters.

Helium will also cool growing clusters, reducing the chance that clusters lose atoms to vaporization from becoming too hot internally during the condensation process. However, this does not appear to lead to larger clusters under these conditions. There is already a large amount of rare gas available to cool growing clusters with argon, and it seems the losses to vaporization are minimal under these conditions. This cooling process may be more important when trying to grow very large clusters and a small addition of helium could be used for this purpose.

The aggregation gas is not required to run the magnetron source. However, adding even a small amount of helium usually provides a noticeable increase in the measured ion current and its use is recommended for most circumstances. 


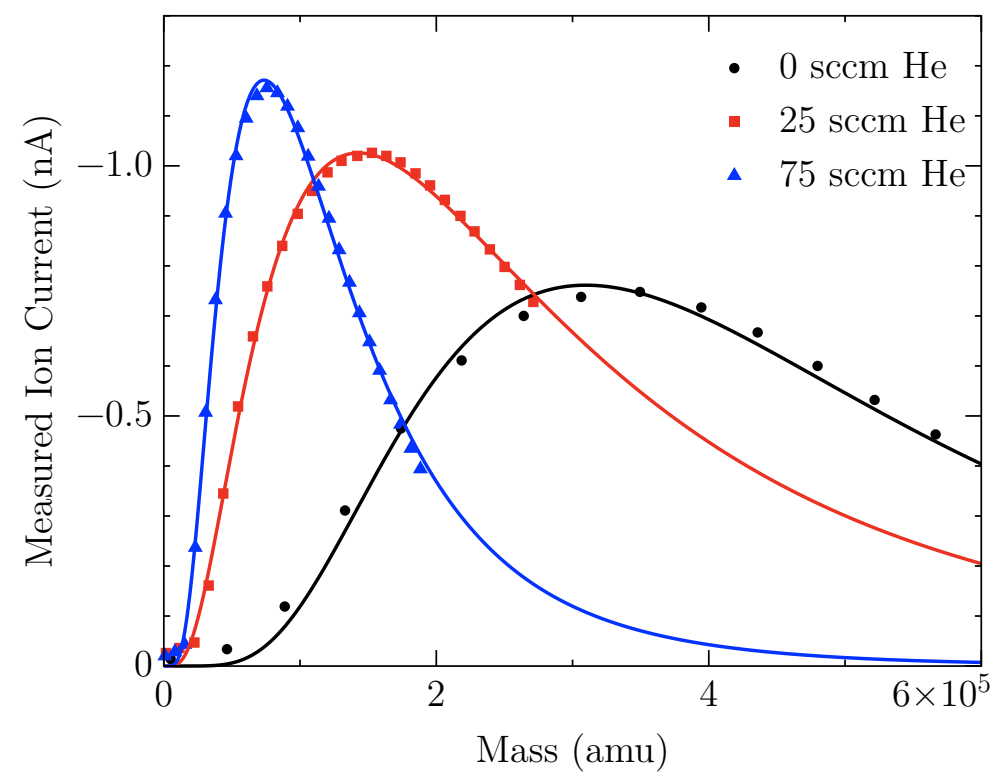

Figure 10.4: Plot of experimental data illustrating the effect of changing aggregation gas (helium) flow on the cluster distribution produced in the magnetron. The points are the experimental data and the matching colored lines are log-normal distribution fits to the data.

\section{Controlling Size Distribution}

By understanding these trends, the cluster mass distribution can be greatly influenced, even without performing any mass selection techniques. Two examples of thoughtfully combining these parameters to create greatly differing cluster beams are demonstrated in Figure 10.5.

In the left panel, I focus on the parameters which will create low mass, small particles. From the prior analysis, this includes a short aggregation distance to limit cluster growth time, a high pressure of helium to increase the number of seeds via termolecular collisions and reduce the number of available atoms per cluster, and low sputtering power and argon pressure to also limit the number of free metal atoms condensing into each cluster. As seen in the figure, the peak of the mass distribution with these conditions is only around $100 \mathrm{Cu}$ atoms per cluster. For a spherical $\mathrm{Cu}$ cluster, this corresponds to a diameter of roughly $1.4 \mathrm{~nm}$. The opposite approach to affect cluster size was applied in the right panel. A long aggregation distance which maximizes the amount of time clusters have to grow, while a higher argon pressure and sputtering power increases the amount of metal atoms available to condense. No helium was added, though a small amount of helium should also improve the condensation rate. With these conditions the cluster mass distribution peaks at over $8000 \mathrm{Cu}$ atoms per cluster, which for spherical $\mathrm{Cu}$ clusters is about $6.3 \mathrm{~nm}$ in size. However, the distribution is also extremely broad - extending to clusters of over 20,000 $\mathrm{Cu}$ atoms (masses of more than 1,000,000 amu per cluster). 

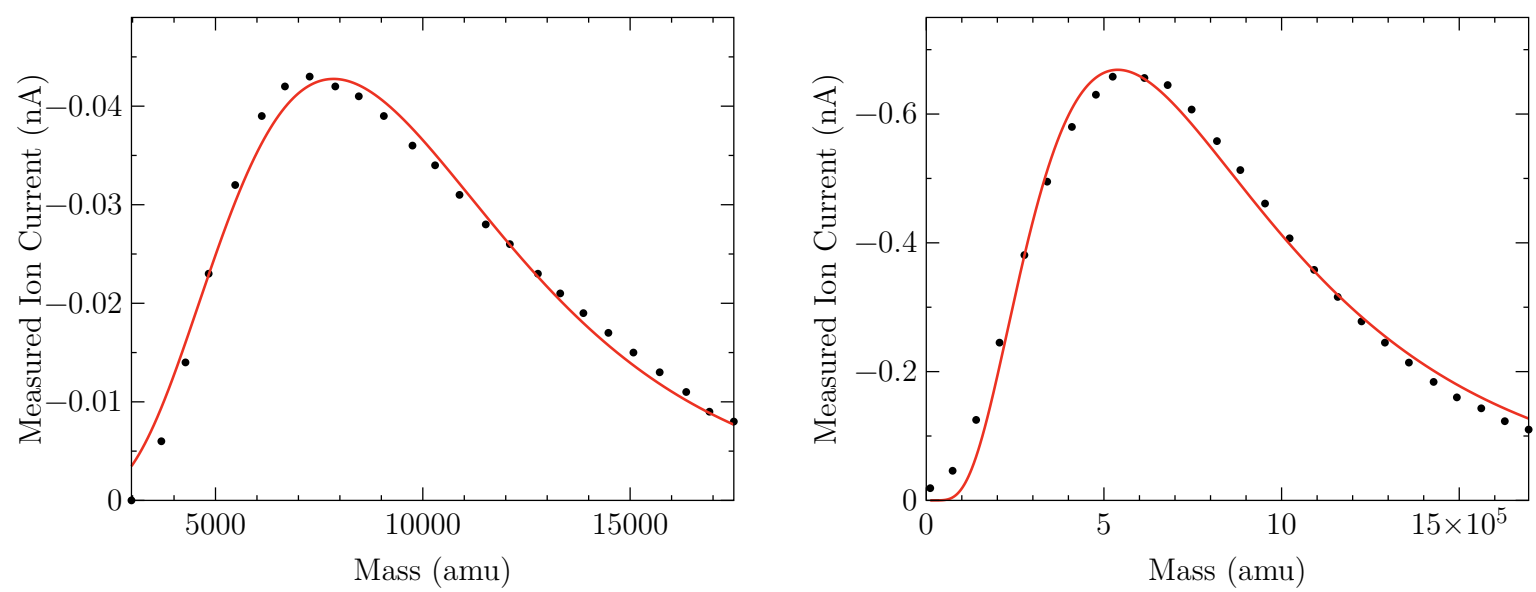

Figure 10.5: Plots of experimental data illustrating the ability of the magnetron source to produce very different size distributions based on different combinations of source parameters. Left: Low mass distribution produced with a short aggregation distance and large helium flow. Right: High mass distribution produced with a long aggregation distance and high sputtering power.

\subsubsection{Effect of Exit Aperture Size}

A final potentially important parameter should be discussed - the size of the exit aperture. Changing the size of this aperture can greatly affect the size distributions of the clusters. The typical exit aperture size used is $3 \mathrm{~mm}$. By placing a larger aperture at the exit, the overall gas pressure in the aggregation region drops significantly for a given flow rate of aggregation gases. This lower working pressure makes it much easier to obtain low mass, small clusters. It can also make it more difficult to sustain the magnetron discharge. In contrast, replacing the thin aperture by a $1 \mathrm{~cm}$ long $3 \mathrm{~mm}$ nozzle, the cluster distribution always trends to very large $>500,000 \mathrm{amu}$ distributions. This is likely due to the nozzle being a high collision region so no combination of sputtering gases or aggregation lengths will prevent significant clustering while the atoms are exiting through the nozzle.

While the exit aperture has a measurable effect, it currently is not an easily adjustable parameter. In order to replace the aperture plate, the entire chamber must be vented and opened. This makes it unsuitable for daily adjustment. There are plans to modify the aperture to make it adjustable in vacuo. If that is implemented, it will likely be one of the most important parameters for routinely adjusting the cluster distribution over a broad size range.

\subsection{Time of Flight Mass Spectrometer}

A custom time of flight mass spectrometer has been built to analyze the mass distribution of the nanoparticle ion beam after mass selection in the quadrupole mass filter. The analyzer's specifications are described in Chapter 9.4. The conversion of flight time into a 
Chapter 10 Characterization and Preliminary Results

mass scale must first be calibrated. This is done for several extraction pulse voltages. The results of this calibration are compared with simulation and basic theory.

\subsubsection{Calibrating the Time-to-Mass Conversion}

In order to convert the recorded time of flight data from the time scale to a mass scale, a calibration curve must be generated. Typically, time of flight mass spectrometry is done on masses which are low relative to the nanoparticle masses the magnetron source generates. In traditional time of flight mass spectrometry, calibration can be achieved by curve fitting the measured flight times for two or more well-known standard masses. However, with the magnetron sputtering source it is not a simple task to produce two precisely known masses in the region of interest. There is the further complication that even if calibration were done with two known masses in the ion beam $(\mathrm{m} / z=4$ and 40 are from ionized helium and argon carrier gases), it would be difficult to evaluate how good the fit is to masses in the $>100,000$ amu range.

To resolve these issues, tantalum was used as the source sputtering material in the magnetron and time of flight spectra recorded for a number of small $\mathrm{Ta}_{n}^{+}$clusters. Tantalum is a useful material for this task as it has only one naturally occurring isotope, ${ }^{181} \mathrm{Ta}$, and is relatively high mass (gold would also be suitable, but is many times more expensive). With only a single isotope, the mass of each subsequent $n$-mer cluster occurs at fixed intervals of $181 \mathrm{amu}$, with no broadening occurring in larger clusters due to mixing isotopes. By recording TOF spectra for clusters up to 18, I am able to obtain many known mass peaks in the range of masses from 181 to $3258 \mathrm{amu}$. This allows a reliable curve fit to be generated over a wide mass range for clusters which are produced in the magnetron sputtering source.

Figure 10.6 shows a representative time of flight spectrum for the series of tantalum clusters from $n=4$ to 18 obtained with an extraction pulse voltage of $2000 \mathrm{~V}$. To extract the time of flight for each cluster, the peaks were fitted to an exponentially modified Gaussian using the multipeak fitting routine in the commercial software Igor Pro 6 from Wavemetrics [27]. Spectra like this were recorded for multiple mass ranges to obtain the time of flight for all clusters between $n=1$ and 18 for four different extraction pulse voltages. The peak times were extracted via the method described above and the results are tabulated in Table 10.1.

From these points, a curve fitting procedure can be performed. As shown in the derivation of the time of flight equation in Chapter 8.4.2, the time should be related to the mass by a function of the form:

$$
t=a \sqrt{m}
$$

The peak mass plotted versus the time of flight for each cluster for all four extraction pulse voltages can be seen in Figure 10.7. The points have been fit to a function of the form above plus an additional variable, $b$, to account for experimental uncertainty in the start time, $t=0$, of the extraction pulse. The equation coefficients $a$ and $b$ determined from this fitting are shown in Table 10.2. 


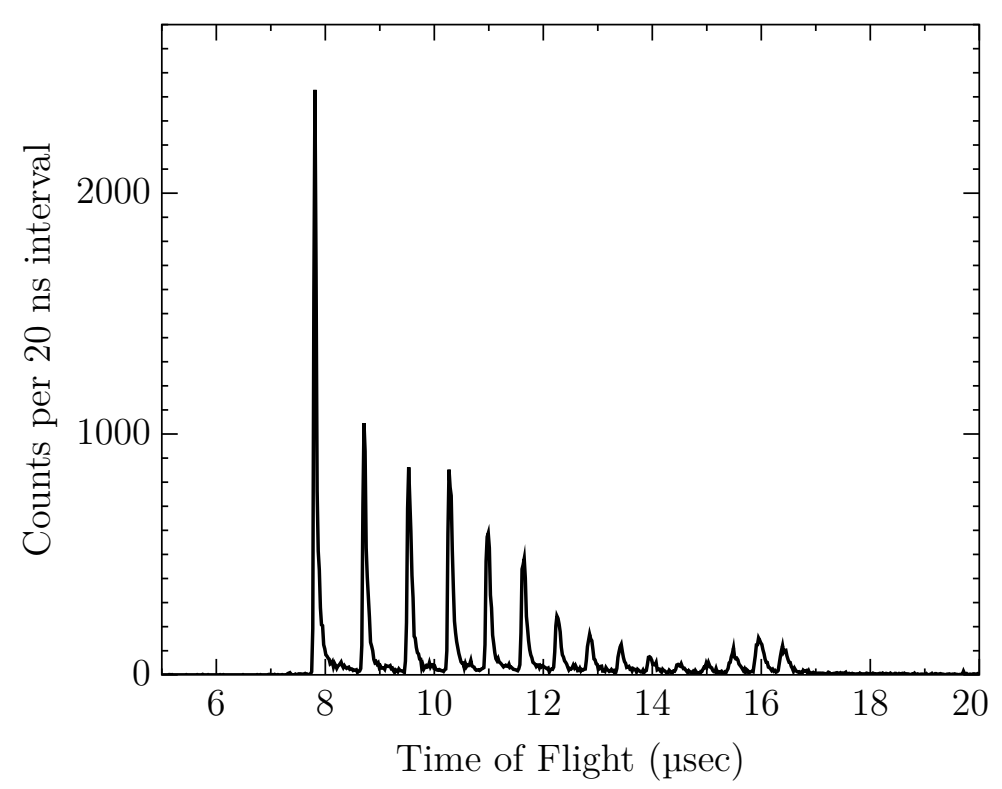

Figure 10.6: Representative $\mathrm{Ta}_{n}^{+}$cluster time of flight spectrum used for calibrating the TOF-to-mass conversion function for $n=4$ to 18 .

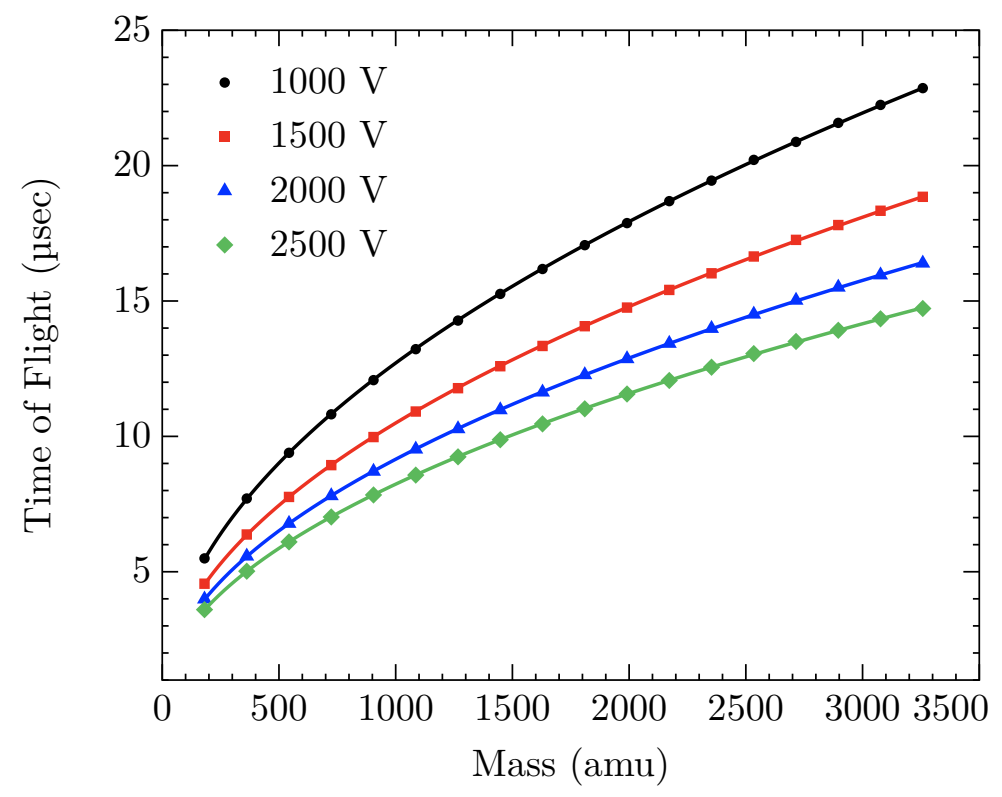

Figure 10.7: Plot of tantalum cluster mass vs measured TOF used to calibrate the TOF-tomass conversion function. The points are the peak times extracted from plots like figure 10.6. The matching colored solid lines are the best fit function to the data points of the form $a \sqrt{m}+b$. 
Chapter 10 Characterization and Preliminary Results

Table 10.1: Calibration time of flight data.

\begin{tabular}{|c|c|c|c|c|c|}
\hline \multirow[b]{2}{*}{ Cluster } & \multirow[b]{2}{*}{$m / z$} & \multicolumn{4}{|c|}{ Time of Flight ( $\mu$ sec) } \\
\hline & & $1000 \mathrm{~V}$ & $1500 \mathrm{~V}$ & $2000 \mathrm{~V}$ & $2500 \mathrm{~V}$ \\
\hline $\mathrm{Ta}^{+}$ & 181 & 5.49 & 4.56 & 3.99 & 3.60 \\
\hline $\mathrm{Ta}_{2}^{+}$ & 362 & 7.70 & 6.38 & 5.57 & 5.02 \\
\hline $\mathrm{Ta}_{3}^{+}$ & 543 & 9.39 & 7.76 & 6.78 & 6.10 \\
\hline $\mathrm{Ta}_{4}^{+}$ & 724 & 10.82 & 8.94 & 7.80 & 7.02 \\
\hline $\mathrm{Ta}_{5}^{+}$ & 905 & 12.08 & 9.97 & 8.71 & 7.84 \\
\hline $\mathrm{Ta}_{6}^{+}$ & 1086 & 13.22 & 10.92 & 9.53 & 8.57 \\
\hline $\mathrm{Ta}_{7}^{+}$ & 1267 & 14.27 & 11.78 & 10.28 & 9.24 \\
\hline $\mathrm{Ta}_{8}^{+}$ & 1448 & 15.26 & 12.59 & 10.98 & 9.88 \\
\hline $\mathrm{Ta}_{9}^{+}$ & 1629 & 16.18 & 13.34 & 11.63 & 10.46 \\
\hline $\mathrm{Ta}_{10}^{+}$ & 1810 & 17.06 & 14.07 & 12.27 & 11.02 \\
\hline $\mathrm{Ta}_{11}^{+}$ & 1991 & 17.88 & 14.76 & 12.86 & 11.57 \\
\hline $\mathrm{Ta}_{12}^{+}$ & 2172 & 18.69 & 15.41 & 13.43 & 12.06 \\
\hline $\mathrm{Ta}_{13}^{+}$ & 2353 & 19.45 & 16.02 & 13.98 & 12.55 \\
\hline $\mathrm{Ta}_{14}^{+}$ & 2534 & 20.21 & 16.64 & 14.51 & 13.06 \\
\hline $\mathrm{Ta}_{15}^{+}$ & 2715 & 20.88 & 17.26 & 15.02 & 13.51 \\
\hline $\mathrm{Ta}_{16}^{+}$ & 2896 & 21.58 & 17.81 & 15.51 & 13.92 \\
\hline $\mathrm{Ta}_{17}^{+}$ & 3077 & 22.24 & 18.33 & 15.96 & 14.34 \\
\hline $\mathrm{Ta}_{18}^{+}$ & 3258 & 22.86 & 18.85 & 16.39 & 14.72 \\
\hline
\end{tabular}


Table 10.2: Best fit parameters to experimental data.

\begin{tabular}{ccc}
\hline Pulse Voltage & $\mathbf{a}$ & $\mathbf{b}$ \\
\hline $1000 \mathrm{~V}$ & 0.40 & .10 \\
$1500 \mathrm{~V}$ & 0.33 & 0.11 \\
$2000 \mathrm{~V}$ & 0.29 & 0.13 \\
$2500 \mathrm{~V}$ & 0.26 & 0.14 \\
\hline
\end{tabular}

To finalize the calibration, a function to convert the time scale into the mass scale needs to be obtained by solving for mass in terms of arrival time. This function will have the form:

$$
m=a t^{2}+b t+c
$$

The coefficients obtained when solving each equation determined from Table 10.2 are compiled in Table 10.3. These are the calibration coefficients used for converting all of the obtained time of flight spectra in this dissertation into a mass scale. One additional mathematical transform needs to be applied when converting from the time scale to a mass scale. The intensity of the time of flight spectra is divided by the value of the derivative with respect to time of the time-to-mass conversion function to determine the signal intensity in the new coordinate space. This is known as a Jacobian transformation and is a well-known mathematical transform for this type of coordinate exchange.

Table 10.3: Time-to-mass conversion equation coefficients.

\begin{tabular}{cccc}
\hline Pulse Voltage & $\mathbf{a}$ & $\mathbf{b}$ & $\mathbf{c}$ \\
\hline $1000 \mathrm{~V}$ & 6.28 & -1.19 & 0.06 \\
$1500 \mathrm{~V}$ & 9.28 & -2.07 & 0.12 \\
$2000 \mathrm{~V}$ & 12.29 & -3.28 & 0.22 \\
$2500 \mathrm{~V}$ & 15.27 & -4.38 & 0.31 \\
\hline
\end{tabular}




\subsubsection{Mass Resolution}

Determining the mass resolution of the time of flight mass analyzer is complicated by the aforementioned lack of a known high-mass standard and the wide distribution of masses which can be present in the ion beam. Ideally, the time of flight analyzer's resolution should be determined without any contribution from the resolution of the quadrupole mass filter. The best choice to examine the mass resolution of the time of flight analyzer is one of the small tantalum clusters used to calibrate the time to mass conversion function. For these clusters, it can be certain that the single peak corresponds to the response from a single known mass. The width of one of these peaks should only be related to the resolution of the analyzer. The $\mathrm{Ta}_{4}^{+}$peak from Figure 10.6 was chosen for this purpose. An enlarged plot of this peak and the standard Gaussian fit are shown in Figure 10.8.

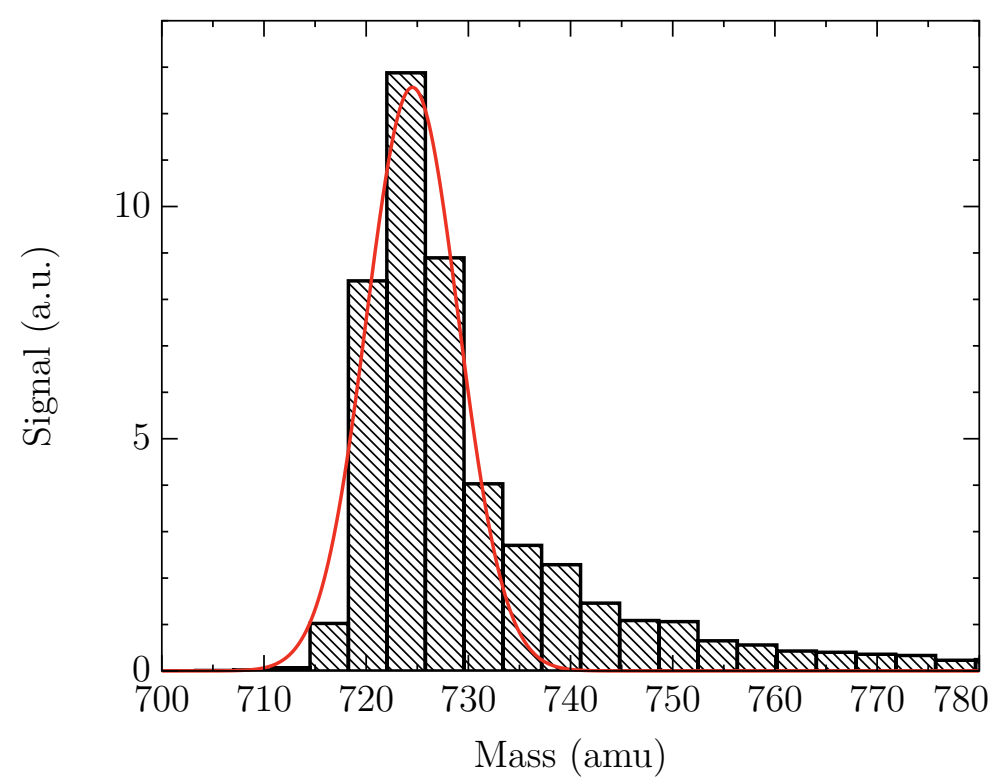

Figure 10.8: Enlarged $\mathrm{Ta}_{4}^{+}$cluster region from Figure 10.6 (converted from TOF to a mass coordinate) to demonstrate experimental mass resolution. The red line is a Gaussian fit to the peak position.

From the fit, the full width at half maximum of the peak is determined to be $10.6 \mathrm{amu}$. The mass of the $\mathrm{Ta}_{4}^{+}$cluster is $724 \mathrm{amu}$, so the apparent mass resolution of the time of flight analyzer is $1.5 \%$. This is better than the value estimated from the previous SIMION simulations which gave an expected resolution of $3 \%$, however that simulation was done for much higher masses. This demonstrates that the resolution of the time of flight analyzer is near expectations and does not perform significantly worse in reality than in the simulation. This resolution should be sufficient to analyze the width of typical mass filtered cluster ion distributions which are typically selected with a $10 \%$ to $20 \%$ resolution. 


\subsubsection{Comparison of Experimental Results to Simulations}

The straight-forward construction of this analyzer lends itself well to examining the performance using simulations and theory.

The exact dimensions of the time of flight analyzer were modeled into SIMION as accurately as possible. 18 ions with masses matching those of the tantalum clusters used for calibrating the time-to-mass conversion in the previous section were run through the simulation and the results are shown numerically in Table 10.4 and graphically in Figure 10.9.

The resulting simulated flight times are very close to the experimental values. As seen in the figure, the simulation data also follows an $a \sqrt{m}$ function. All of the simulated times are about 100 nsec shorter than the actual experiment times. There are two possible reasons for this. First, the real system could have some imperfection which just is not modeled correctly in the idealized SIMION model. Second, the calibration function is actually of the form $a \sqrt{m}+b$. This $b$ term is effectively some time offset from when the trigger is sent to when the pulse voltage is actually applied. This leads to some dead time in the time of flight where the data recording has been triggered, yet no pulse voltage has actually been applied. This $t_{0}$ offset of the trigger before the pulse is applied is noticeable on an oscilloscope and is around 160 nsec. This is not necessarily the exact time offset between when the multichannel scaler receives the trigger and begins recording. The best fit function found in the previous section determines the offset for a $2000 \mathrm{~V}$ pulse to be around $133 \mathrm{nsec}$. This value is consistent with the trigger offset seen on the oscilloscope. The lower panel in Figure 10.9 shows a comparison of the data when this dead time is removed from the experimental time of flight (equivalently one could add it to the simulation time). In this case, the average deviation of the simulation to the experiment drops to $24.3 \mathrm{nsec}$. Another factor which could lead to deviations of the simulation to experimental times is the finite rise time of the high voltage pulse in the experiment. In simulation, the pulse is either on or off, with no value in between. The actual electronics have a rise time to the full pulse voltage of $\approx 10 \mathrm{nsec}$, which can lead to a slower than expected acceleration in the initial stages of the pulse region.

One final comparison to theoretical performance that can be done is calculating a time of flight from the fundamental equation derived in Chapter 8. Calculating the times in the acceleration region and field-free region are performed as described previously with the distances between electrodes and other elements of construction being precisely as measured in the instrument. An additional component to the equation needs to be added to account for the acceleration to the detector after the field free flight. Writing an equation for time in the third biased detector region is a bit complicated as the accelerating field in this region is inhomogenous. I approximated this field with one which exponentially increases as the ion approaches the detector. The results of this calculation can be seen in Table 10.5.

The calculated times agree very well with the experimental times, with most differences being less than 20 nsec. However, this is with uncorrected experimental flight times which include the $t_{0}$ offset discussed above. Taking this into account, these calculated flight times are actually $100-120$ nsec too long. The agreement is still excellent, considering the simple 
Chapter 10 Characterization and Preliminary Results

Table 10.4: Comparison of experimental flight times to simulation.

\begin{tabular}{ccccc}
\hline Cluster & $\boldsymbol{m} / \boldsymbol{z}$ & $\begin{array}{c}\text { Exp. TOF } \\
(\mathrm{amu})\end{array}$ & $\begin{array}{c}\text { Sim. TOF }) \\
(\mu \mathrm{sec})\end{array}$ & Difference \\
\hline $\mathrm{Ta}^{+}$ & 181 & 3.99 & 3.85 & 0.14 \\
$\mathrm{Ta}_{2}^{+}$ & 362 & 5.57 & 5.44 & 0.13 \\
$\mathrm{Ta}_{3}^{+}$ & 543 & 6.78 & 6.66 & 0.12 \\
$\mathrm{Ta}_{4}^{+}$ & 724 & 7.80 & 7.69 & 0.11 \\
$\mathrm{Ta}_{5}^{+}$ & 905 & 8.70 & 8.60 & 0.10 \\
$\mathrm{Ta}_{6}^{+}$ & 1086 & 9.52 & 9.42 & 0.10 \\
$\mathrm{Ta}_{7}^{+}$ & 1267 & 10.28 & 10.18 & 0.10 \\
$\mathrm{Ta}_{8}^{+}$ & 1448 & 10.98 & 10.88 & 0.10 \\
$\mathrm{Ta}_{9}^{+}$ & 1629 & 11.63 & 11.54 & 0.10 \\
$\mathrm{Ta}_{10}^{+}$ & 1810 & 12.27 & 12.16 & 0.11 \\
$\mathrm{Ta}_{11}^{+}$ & 1991 & 12.86 & 12.76 & 0.10 \\
$\mathrm{Ta}_{12}^{+}$ & 2172 & 13.43 & 13.32 & 0.11 \\
$\mathrm{Ta}_{13}^{+}$ & 2353 & 13.98 & 13.87 & 0.11 \\
$\mathrm{Ta}_{14}^{+}$ & 2534 & 14.51 & 14.39 & 0.12 \\
$\mathrm{Ta}_{15}^{+}$ & 2715 & 15.02 & 14.90 & 0.12 \\
$\mathrm{Ta}_{16}^{+}$ & 2896 & 15.51 & 15.39 & 0.12 \\
$\mathrm{Ta}_{17}^{+}$ & 3077 & 15.96 & 15.86 & 0.10 \\
$\mathrm{Ta}_{18}^{+}$ & 3258 & 16.39 & 16.32 & 0.07 \\
\hline & & & & \\
\hline
\end{tabular}



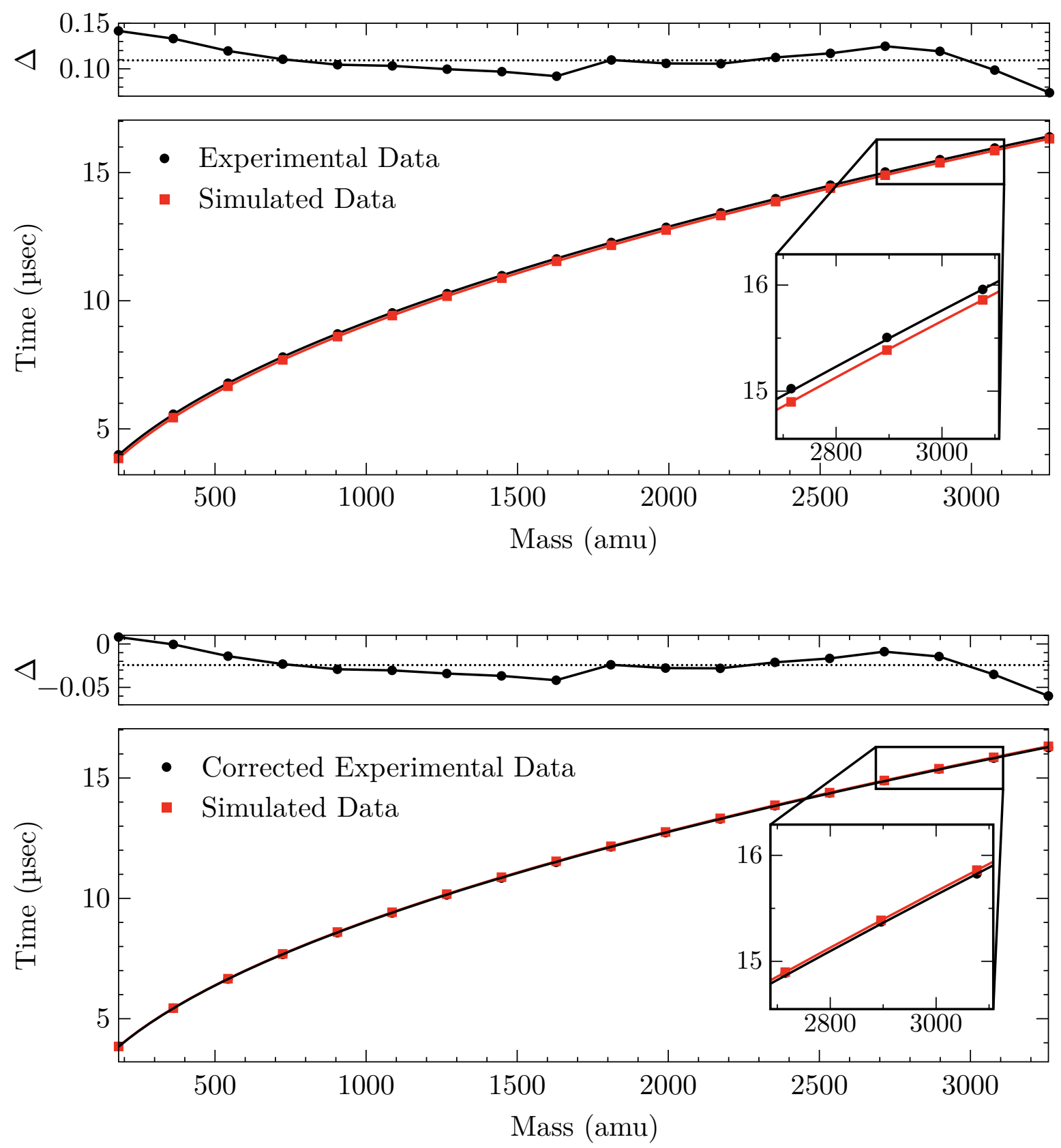

Figure 10.9: Plots of experimental and simulated data for comparison. The top panel shows raw experimental data (black) compared to a SIMION generated TOF (red) for identical masses. The difference between experimental data and simulated data are plotted on an additional axis above. The bottom panel shows the same comparison when the experimental data has the $b$ term of the fit function subtracted. 
Chapter 10 Characterization and Preliminary Results

Table 10.5: Comparison of experimental flight times to theoretically calculated flight times.

\begin{tabular}{ccccc}
\hline Cluster & $\boldsymbol{m} / \boldsymbol{z}$ & $\begin{array}{c}\text { Exp. TOF } \\
(\mathrm{amu})\end{array}$ & $\begin{array}{c}\text { Calc. TOF }) \\
(\mu \mathrm{sec})\end{array}$ & Difference \\
\hline $\mathrm{Ta}^{+}$ & 181 & 3.99 & 3.87 & 0.12 \\
$\mathrm{Ta}_{2}^{+}$ & 362 & 5.57 & 5.48 & 0.09 \\
$\mathrm{Ta}_{3}^{+}$ & 543 & 6.78 & 6.71 & 0.07 \\
$\mathrm{Ta}_{4}^{+}$ & 724 & 7.80 & 7.75 & 0.05 \\
$\mathrm{Ta}_{5}^{+}$ & 905 & 8.71 & 8.66 & 0.05 \\
$\mathrm{Ta}_{6}^{+}$ & 1086 & 9.53 & 9.49 & 0.04 \\
$\mathrm{Ta}_{7}^{+}$ & 1267 & 10.28 & 10.25 & 0.03 \\
$\mathrm{Ta}_{8}^{+}$ & 1448 & 10.98 & 10.96 & 0.02 \\
$\mathrm{Ta}_{9}^{+}$ & 1629 & 11.63 & 11.62 & 0.01 \\
$\mathrm{Ta}_{10}^{+}$ & 1810 & 12.27 & 12.25 & 0.02 \\
$\mathrm{Ta}_{11}^{+}$ & 1991 & 12.86 & 12.85 & 0.01 \\
$\mathrm{Ta}_{12}^{+}$ & 2172 & 13.43 & 13.42 & 0.01 \\
$\mathrm{Ta}_{13}^{+}$ & 2353 & 13.98 & 13.97 & 0.01 \\
$\mathrm{Ta}_{14}^{+}$ & 2534 & 14.51 & 14.50 & 0.01 \\
$\mathrm{Ta}_{15}^{+}$ & 2715 & 15.02 & 15.00 & 0.02 \\
$\mathrm{Ta}_{16}^{+}$ & 2896 & 15.51 & 15.50 & 0.01 \\
$\mathrm{Ta}_{17}^{+}$ & 3077 & 15.96 & 15.97 & -0.01 \\
$\mathrm{Ta}_{18}^{+}$ & 3258 & 16.39 & 16.44 & -0.05 \\
\hline & & & & \\
\hline
\end{tabular}


model used and the approximation of the behavior in the detector region.

The strong agreement between numerical and analytical simulation and experiment provides confidence in the accuracy and reliability of this mass analyzer over a wide mass range.

\subsection{Quadrupole Mass Filter}

In this section, the performance of the quadrupole mass filter for cluster size selection is characterized, revealing a large deviation between the mass selected by the voltage and frequency on the quadrupole and the actual mass transmitted. This deviation is experimentally quantified and a correction factor for the quadrupole's transmitted mass is determined.

\subsubsection{Quadrupole Mass Filter's Transmitted Mass Resolution}

For determining the resolution performance of the quadrupole mass filter, one set of frequency and voltage settings was chosen, corresponding to a peak mass at 118,000 amu. Time of flight spectra were taken with the quadrupole set to $100 \%$ resolution and $10 \%$ resolution. The resulting spectra can be seen in Figure 10.10.
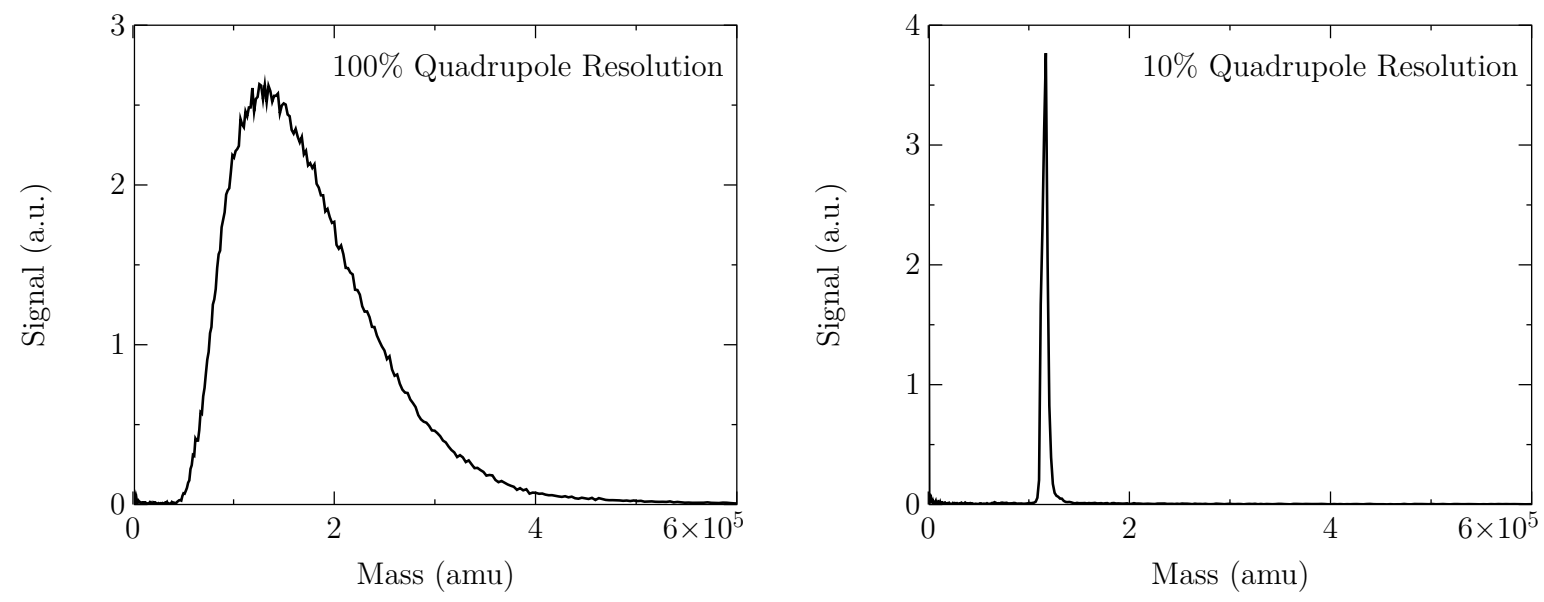

Figure 10.10: Plots of experimentally measured mass distributions for two settings of quadrupole resolution. Left: Quadrupole resolution set to 100\%. Right: Quadrupole resolution set to $10 \%$.

Analysis of the $100 \%$ resolution peak shows:

$$
\frac{m}{\Delta m}=\frac{134820 \mathrm{amu}}{138630 \mathrm{amu}}=0.97
$$


while analysis of the $10 \%$ resolution peak shows:

$$
\frac{m}{\Delta m}=\frac{115920 \mathrm{amu}}{6548 \mathrm{amu}}=17.7
$$

The $100 \%$ resolution setting is essentially exactly as expected with the $m / \Delta m \approx 1$. The $10 \%$ resolution setting actually has a better than expected $m / \Delta m=17.7$, corresponding to $\Delta m / m=5.6 \%$. This demonstrates that the resolution of the quadrupole behaves as expected for controlling the width of the mass distribution in the ion beam and that the goal of depositing size selected nanoparticles with a narrow size distribution is possible using this instrument. Keep in mind that the size of the cluster should scale with the cube root of the mass, so a $5-10 \%$ mass resolution corresponds to a $1.7-2.1 \%$ size resolution.

\subsubsection{Comparison of Time of Flight Results to Quadrupole Performance}

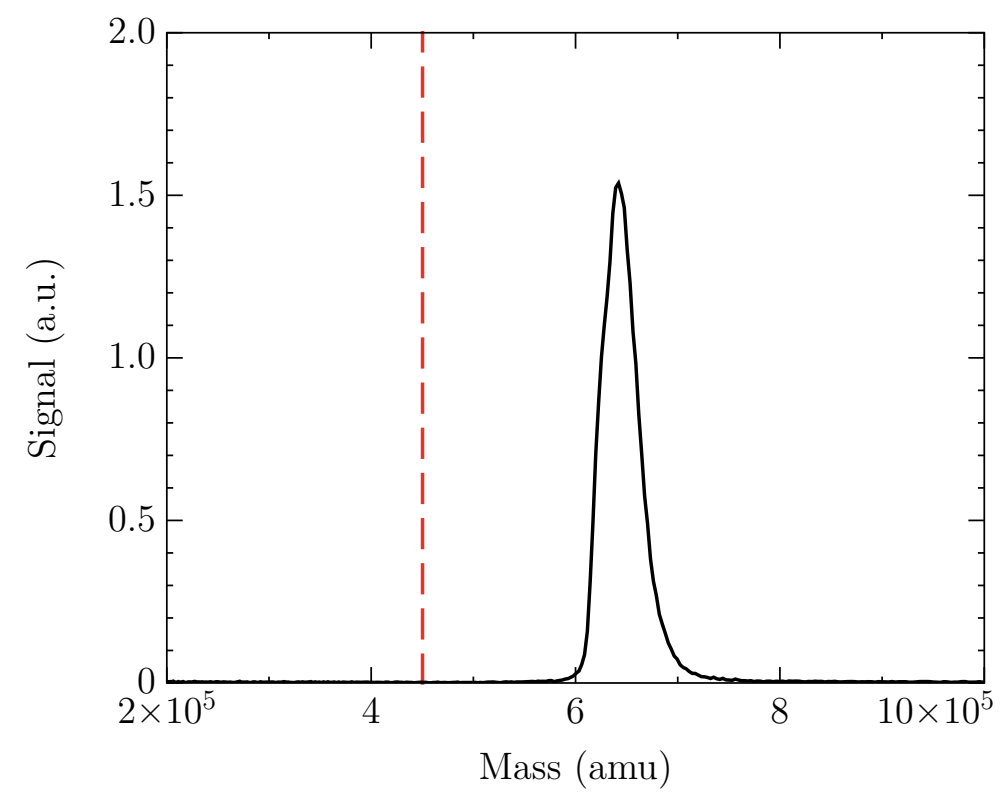

Figure 10.11: Plot of measured mass distribution illustrating the wide difference between the actually transmitted mass versus the mass selected on the quadrupole controller. The dashed red line is the mass which was selected on the quadrupole.

After calibrating the time of flight mass spectrometer, one performance issue which becomes immediately apparent is a large offset (around 40\%) between the desired mass chosen with the quadrupole and the actual transmitted mass. An example of this can be seen in Figure 10.11. In this figure, a recorded TOF spectrum for a mass reported to be 450,000 amu on the quadrupole controller's front panel has been converted into a mass 
scale. The selected mass position is marked by a dashed line in the figure. As can be seen, the offset from the experimentally determined mass is very significant.

The offset of the transmitted mass from selected mass is not completely unexpected. Oxford Applied Research, the company who produces the quadrupole mass filter, allows for an adjustable correction factor, $k$, in their controller software [45]. The transmitted mass shown on the quadrupole controller front panel is simply calculated from the set RF voltage and frequency via the equation described previously in Chapter 9.3. With the time of flight mass spectrometer accurately calibrated over a wide mass range, it is possible to calibrate the correction factor, $k$, of the quadrupole by comparing the TOFMS observed mass to the mass calculated from this equation.

\subsubsection{Calibration of the Quadrupole Mass Filter}

In order to calibrate the quadrupole mass filter and determine what the value of $k$ should be, time of flight mass spectra for a large series of tantalum anion clusters, $\mathrm{Ta}_{n}^{-}$, were recorded with a $10 \%$ transmitted mass resolution on the quadrupole. These spectra were then fit to an exponentially modified Gaussian through a peak fitting routine in the commercial software package Igor Pro [27]. The exponentially modified Gaussian function was needed to accurately fit the tail end of most peaks, though the origin of this shape is not apparent. This peak fitting routine determines the TOF where the maximum of the peak intensity is. The determined time of flight from each spectra was then converted into a mass through the equations determined in the previous section. This results in an experimentally determined mass for each mass selected via the quadrupole controller's front panel. By examining a large number of points over a wide range, a clear deviation from the ideal 1:1 correspondence is observed. Table A.1 tabulates all of the data points obtained in this experiment, and can be found in the Appendix. Figure 10.12 shows these results plotted as experimentally observed mass versus selected mass.

In the figure, a dashed line shows the theoretical 1:1 correspondence between selected mass and transmitted mass when $k=1.0$. The actual ratio between the two is still linear, however a scaling factor is observed for all data points. A best fit line to these points determines the slope, which in turn is exactly the correction factor, $k$. From these results, the correction factor required for our quadrupole mass filter is $k=1.42$. This is a significant correction which could easily have been overlooked without a method for independently verifying cluster masses. Without the time of flight mass analysis, there is not a good method to precisely determine what the correction factor should be, or even determine if there should be one. It might be possible to see larger than expected clusters once deposited on surfaces through various microscopy methods, however, this would not be as accurate a method for determining the correction factor. For example, in particle height analysis from AFM, the shape of the particle on the surface may not be spherical, which could affect the results. In addition, removing the deposition substrate to atmosphere for analysis could induce oxide layer growth which leads to even larger particles.

A final point of note, the near perfect linearity of the plot in Figure 10.12 inspires confidence that the calibration method used for the time of flight analyzer is sound. If 
Chapter 10 Characterization and Preliminary Results

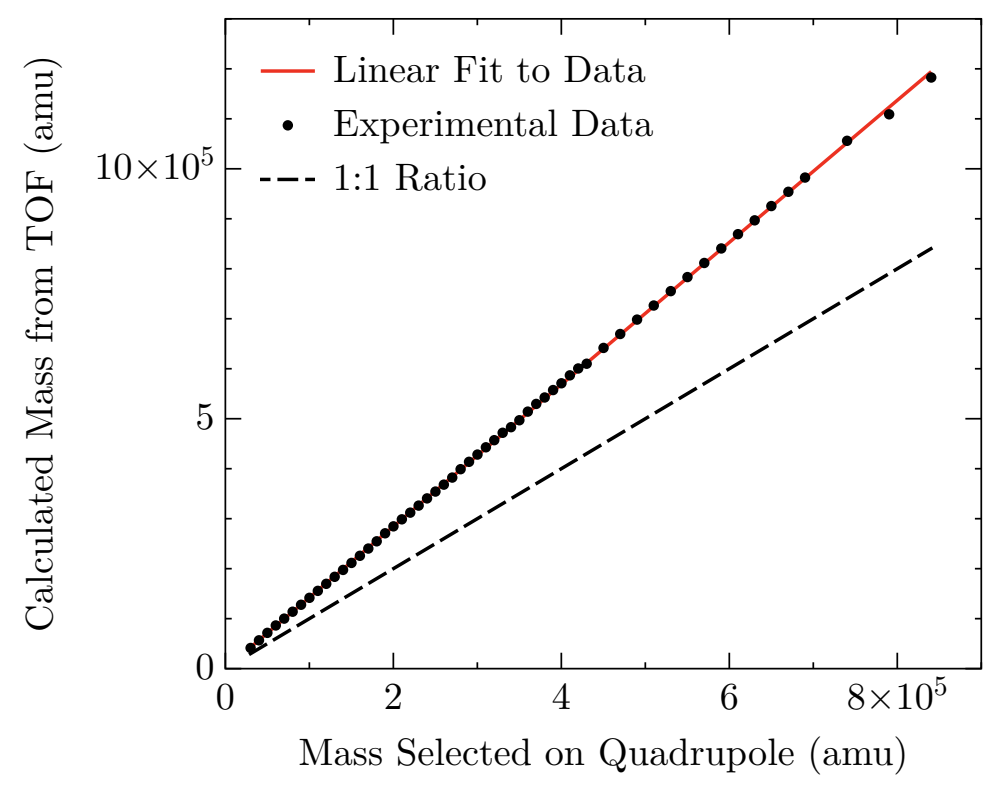

Figure 10.12: Plot of the mass determined by TOF analysis versus the mass selected on the quadrupole filter. The black points are the peak positions determined from mass converted TOF spectra like Figure 10.11. The solid red line is the linear best fit to these points. For comparison, the dashed line shows the 1:1 ratio expected for a TOF analyzer mass which matches the quadrupole selected mass. 
very large masses began to deviate from the calibration curve, then it would be expected that the mass calculated from that curve versus the selected quadrupole mass would show a different trend in the higher masses. In other words, the points in Figure 10.12 would not all be fit by the linear equation, but begin to deviate at some higher mass values. Since that is not observed, any trend away from the calibration curve is likely to be quite small over the mass range of interest and there is more confidence that the TOF mass analysis is reasonably accurate in determining the transmitted mass of clusters.

\subsection{Characterization of Ion Beam}

An experiment was performed to determine the approximate diameter of the mass filtered ion beam and what effect the einzel lens focusing has on the size and intensity of the beam. For this experiment, a Faraday cup with a $2 \mathrm{~mm}^{2}$ area entrance aperture was attached to the end of a linear motion drive mounted perpendicular to the ion beam just after the DN40 CF flange at the quadrupole exit. This Faraday cup could be moved back and forth across the ion beam and the current recorded as a function of the position. Several recorded data sets can be seen in Figure 10.13. The $x$-axis of this plot is given by the position indicator on the linear motion feedthrough. The feedthrough has a travel range from 0 to $50 \mathrm{~mm}$ and the Faraday cup aperture is roughly in the center of the chamber at $25 \mathrm{~mm}$ on this indicator.
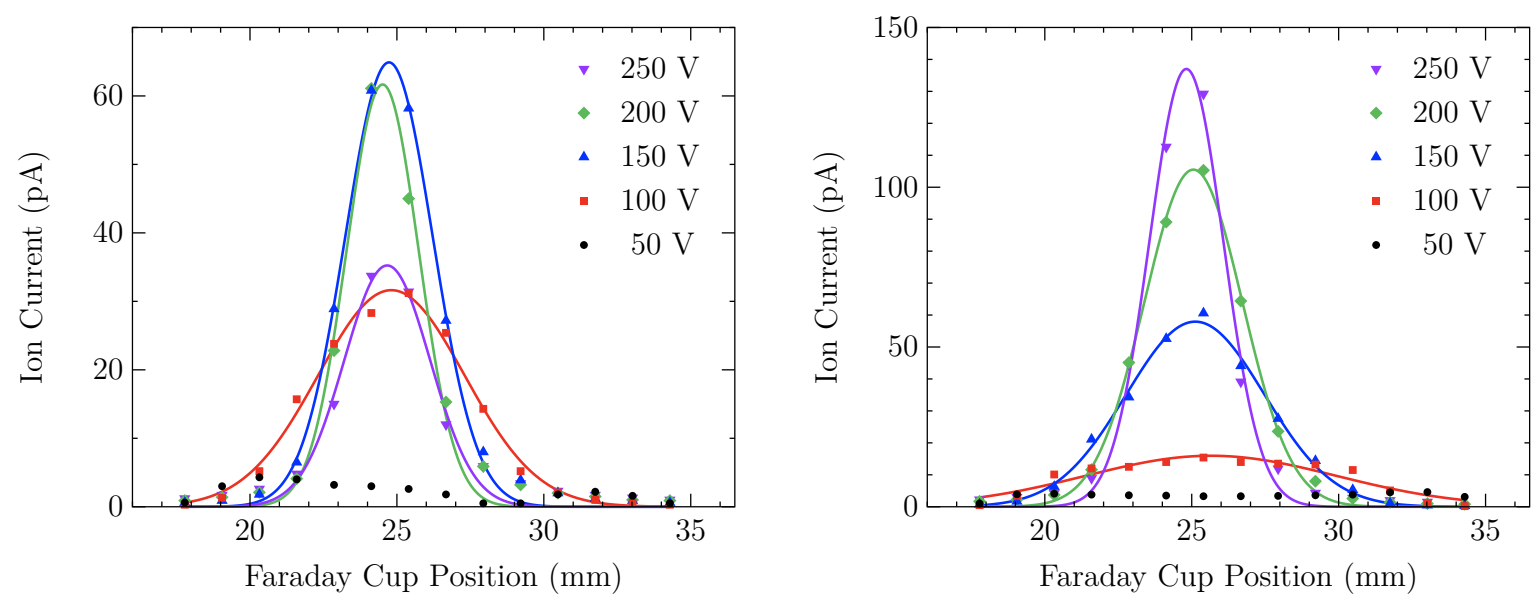

Figure 10.13: Plots of experimental data showing the width of the nanoparticle ion beam for different einzel lens voltages. The Faraday cup position is given as the position on the indicator ruler of the linear motion drive which the Faraday cup was mounted on. The experimental data is shown as points and the matching colored lines are Gaussian fits to the data points (where possible). Left: 150,000 amu clusters. Right: 250,000 amu clusters.

The left panel shows results for a filter mass of 150,000 amu and the right panel is for 250,000 amu. For both masses, with no or low einzel lens voltage, there is essentially 
no measurable ion current. As the einzel lens voltage is increased, the width of the ion beam becomes narrower and the intensity increases. For the 150,000 amu data, there is some maximum focus position between 150 and $200 \mathrm{~V}$ (the blue and green curves) and by $250 \mathrm{~V}$ (the purple curve) the beam intensity goes down. For the 250,000 amu, the intensity continues to increase all the way to $250 \mathrm{~V}$ which is the maximum the DC power supply can provide. This shows that the heavier clusters require a higher potential on the einzel lens to be focused to the same position, which is physically reasonable if the kinetic energy scales with the mass. The width of the ion beam when well focused is reasonably small - between 3 and $5 \mathrm{~mm}$ in diameter. The results here show that the einzel lens after the quadrupole is absolutely essential to obtaining a high intensity and narrow beam.

\subsection{Characterization of the Cluster Ion Energy}

Control over the impact energy the cluster has with the support surface is an important element of this deposition system. At high impact energies, the cluster can be deformed, fragment, or cause damage to the support surface. In general, these are undesirable results. With low impact energy, the ions should not undergo significant structural deformation and not cause damage to the support. This low energy deposition is referred to as "soft landing" of the ions [22]. Typically, the soft landing energy region is on the order of 0.1 to $1 \mathrm{eV}$ per cluster atom. High impact energy is typically considered to be more that $10 \mathrm{eV}$ per cluster atom. In the so-called medium energy region, between 1 and $10 \mathrm{eV}$, it may be possible to create small defects in the support surface without strongly deforming the cluster. This can have the benefit of "pinning" the cluster to the support and reducing the diffusion and aggregation of the clusters on the support [56]. Understanding and control of the cluster deposition energy allows the flexibility to soft land the clusters, pin the clusters to a support at medium energies, or strongly adhere the clusters and produce durable thin films by high energy impact. A number of studies of the effect of cluster energy on landing morphology have been published [6, 21, 28].

In order to control the deposition energy, understanding the kinetic energy distribution of the cluster ion beam exiting the source is necessary. A retarding field energy analyzing Faraday cup was designed and built for this purpose. The energy distribution of the cluster ions was measured as a function of source and mass filter conditions for a wide range of cluster masses. From these results, a theoretical model is developed to explain the observations and assist in future experiments which require precise control of the cluster deposition energy.

\subsubsection{Design and Construction of the Retarding Field Energy Analyzer}

There are several methods which could be used to determine the kinetic energy of the ion beam. The simple method of a plate Faraday cup coupled with retarding field grids was chosen for its quick construction and implementation time, ease of integrating into 
many positions in the instrument, and straight-forward interpretation of data. The design consists of a series of three high transmission mesh grids mounted in front of a solid metal plate connected to a picoammeter for measuring the current of ions impacting the plate. The first grid is grounded to provide a well defined, homogeneous electric field environment. The second grid is used for the retarding field. As a negative bias potential is applied to this grid, anions whose kinetic energy is less than the bias will be repelled and unable to reach the collector plate. The third grid is used as a suppression grid with a $+50 \mathrm{~V}$ bias.

The grids are made of $90 \%$ transmission mesh spot welded to thin $(1 \mathrm{~mm})$ stainless steel plates. The plates are fixed in place by three aluminum oxide ceramic spacers around M2 threaded rods. The entire assembly is mounted to a DN40 CF flange with three BNC electrical feedthroughs. The electrical connections are made by spot welded Kapton coated wire connected to the vacuum side of $\mathrm{BNC}$ electrical feedthroughs by BeCu connectors. The current carrying wire from the Faraday cup is additionally isolated by Kapton coated coaxial shielded wire.

\subsubsection{Operation and Data Analysis}

The cluster ion beam kinetic energy distribution can be measured by applying a retarding potential and recording the ion current on the Faraday cup collector plate as a function of this applied voltage. A suppression voltage can be applied to the last grid to suppress losses by secondary electron emission. The potentials are applied by a simple variable resistor voltage divider powered by a $250 \mathrm{~V}$ maximum power supply (TTi PLH250-P). The current on the Faraday cup plate is measured on a Keithley 6487 Picoammeter. As the retarding potential increases, ions with less kinetic energy than the applied potential are rejected and the observed ion current decreases. The resulting plot of retarding potential versus measured ion current has a sigmoidal shape which can be well fit by the error function:

$$
\operatorname{erf}(z)=\frac{2}{\sqrt{\pi}} \int_{0}^{z} e^{-z^{2}} \mathrm{~d} z, \text { where } z=\frac{(x-\mu)}{\sqrt{2} \sigma}
$$

The derivative of this function is a Gaussian distribution located at $\mu$ with width $\sigma$. This form of a fitting function makes the determination of the mean energy and the full width at half maximum of the energy distribution a simple matter. The data fitting was performed using the curve fitting routine in Igor Pro [27] with the custom functional form:

$$
f(x)=y_{0}+A \times \operatorname{erf}\left(\frac{x-\mu}{\operatorname{abs}(\sigma) \sqrt{2}}\right)
$$

where $y_{0}$ allows for a contribution from background, $A$ affects the direction and magnitude of the fit, and $\mu$ and $\sigma$ are the Gaussian location and width parameters. As the magnitude of the ion current varies based on the mass and source conditions, the ion current plots are normalized to 1 by dividing by the maximum current recorded for a given measurement to simplify the comparison between results. 


\subsubsection{Ion Beam Kinetic Energy Measurements}

\section{Effect of Argon Flow on Kinetic Energy Distribution}

The effect the argon gas flow rate in the cluster source has on the ion kinetic energy for a given mass was investigated. 10 masses of tantalum cluster were selected by the quadrupole mass filter over a wide mass range with a narrow resolution (20\%) and the ion current recorded as a function of the retarding field potential for each mass. For the same 10 masses, this data was re-recorded after changing the argon flow. In all, 30 data points were recorded - 10 points at each flow rate of $15 \mathrm{sccm}, 20 \mathrm{sccm}$, and $25 \mathrm{sccm}$. A representative example of the resulting data plot, the error function fit, and the derived Gaussian energy distribution are shown in Figure 10.14. From the Gaussian, the mean energy and FWHM of the distributions can easily be extracted. The results are summarized in Table 10.6.

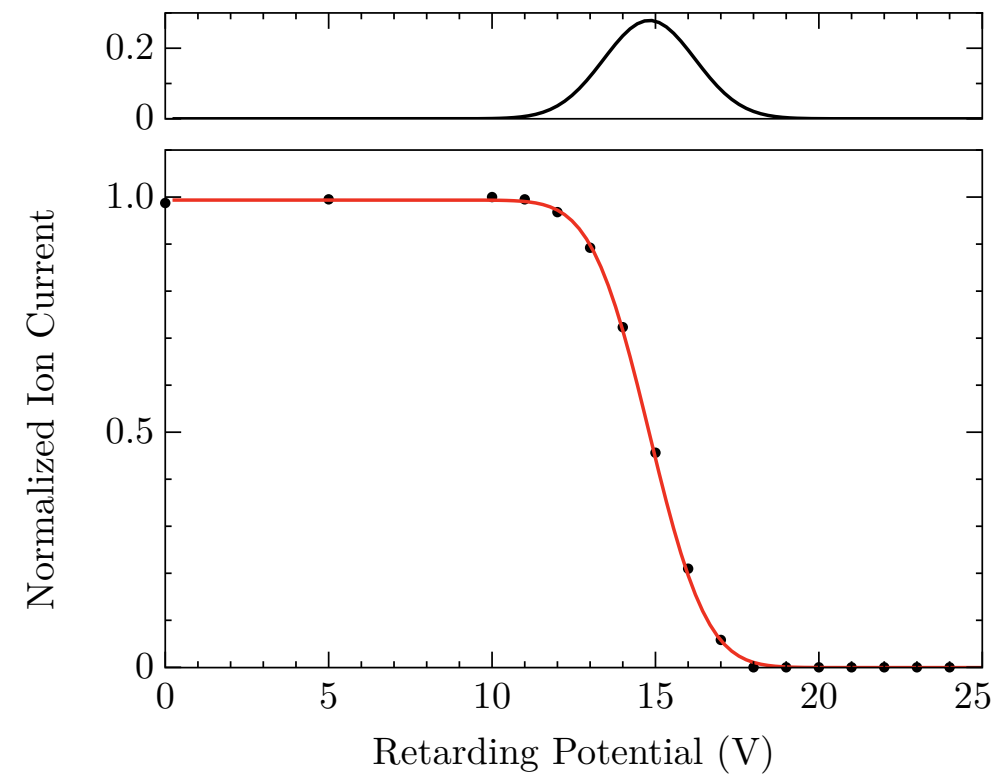

Figure 10.14: Plot of experimental data from the retarding field energy analyzer. Ion current has been normalized to the maximum measured current in a data set. The black points are the experimentally measured ion currents as a function of increasing retarding voltage. The red line is an error function fit to the data points. The corresponding Gaussian energy distribution is plotted on an additional axis above.

A plot of the mean energy versus mass for each of the three flow rates can be seen in Figure 10.15. The results show the cluster energy increases linearly with the mass over this mass range and the slope of the linear fit increases with increasing argon flow rates. The first three points in the $20 \mathrm{sccm}$ flow plot deviate from the rest of the recorded data. I believe this is likely the result of these points being measured after the $25 \mathrm{sccm}$ flow rate data. The pressure in the source may have had a slow response to returning to a lower pressure condition leading to a slow drift in the first recorded points. At first glance, the 
10.5 Characterization of the Cluster Ion Energy

Table 10.6: Retarding field energy analyzer extracted fit for the effect of argon flow rate on cluster energy.

\begin{tabular}{cccccccccc}
\hline & \multicolumn{3}{c}{$\mathbf{1 5} \mathbf{~ s c c m}$} & \multicolumn{3}{c}{ 20 sccm } & \multicolumn{3}{c}{$\mathbf{2 5} \mathbf{~ s c c m}$} \\
\cline { 2 - 10 } Mass & $\mu$ & $\sigma$ & $v$ & $\mu$ & $\sigma$ & $v$ & $\mu$ & $\sigma$ & $v$ \\
$(\mathrm{amu})$ & $(\mathrm{eV})$ & $(\mathrm{eV})$ & $\left(\mathrm{m} \mathrm{s}^{-1}\right)$ & $(\mathrm{eV})$ & $(\mathrm{eV})$ & $\left(\mathrm{m} \mathrm{s}^{-1}\right)$ & $(\mathrm{eV})$ & $(\mathrm{eV})$ & $\left(\mathrm{m} \mathrm{s}^{-1}\right)$ \\
\hline 107,448 & 9.7 & 1.1 & 131.7 & 11.6 & 0.9 & 144.4 & 14.2 & 1.9 & 159.4 \\
119,718 & 10.5 & 1.2 & 130.1 & 12.5 & 1.2 & 141.7 & 15.6 & 1.8 & 158.7 \\
134,217 & 11.4 & 1.3 & 127.8 & 12.8 & 1.4 & 135.4 & 17.0 & 1.6 & 156.5 \\
151,518 & 12.4 & 1.3 & 125.6 & 15.6 & 1.5 & 140.9 & 18.2 & 2.2 & 152.4 \\
172,394 & 13.6 & 1.3 & 123.3 & 17.2 & 1.5 & 138.6 & 20.4 & 2.1 & 150.9 \\
197,901 & 14.9 & 1.4 & 120.3 & 18.8 & 1.7 & 135.4 & 22.3 & 2.3 & 147.4 \\
229,519 & 16.5 & 1.7 & 117.7 & 21.1 & 1.9 & 133.0 & 25.4 & 2.2 & 146.1 \\
269,366 & 18.5 & 2.1 & 115.1 & 24.0 & 2.0 & 131.0 & 28.8 & 2.2 & 143.6 \\
320,568 & 21.1 & 2.4 & 112.6 & 27.3 & 2.4 & 128.2 & 32.5 & 2.2 & 139.8 \\
387,887 & 24.1 & 2.9 & 109.5 & 31.8 & 3.1 & 125.8 & 36.8 & 2.7 & 135.3 \\
\hline
\end{tabular}


observed result here suggests that the cluster velocity may be constant as a result of being carried in a beam of argon. To check this, the mean energy of the cluster is converted to a velocity by the equation for kinetic energy:

$$
E_{T}=\frac{1}{2} m v^{2}
$$

The resulting velocities are shown plotted as a function of mass in Figure 10.15 and can also be found in Table 10.6. The cluster velocities are roughly similar for a given flow of argon, but the heavy clusters are slower than the light clusters in all flow conditions. This suggests that there is a velocity slip which is greater for heavier clusters. This is a well known effect in molecular beam physics and is consistent with the idea that the cluster kinetic energy is mainly derived by acceleration in the carrier gas, and not by some electrostatic acceleration.
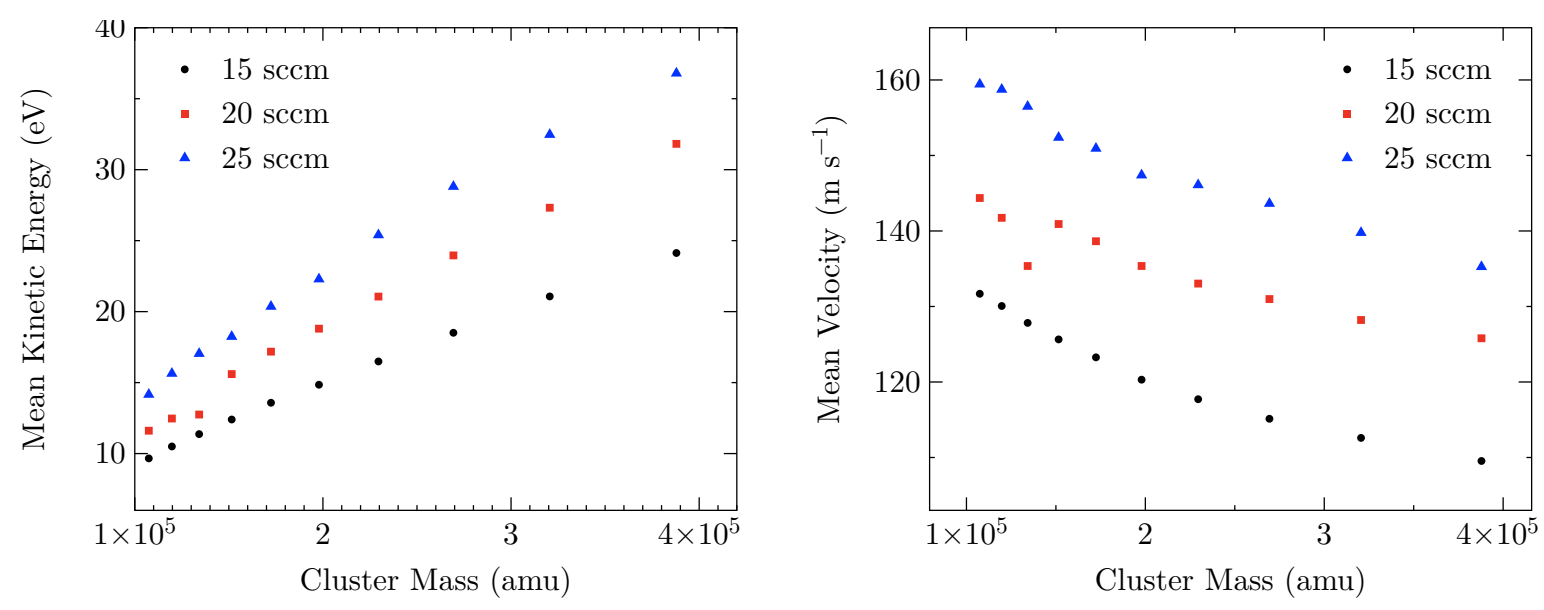

Figure 10.15: Left: Plot of the mean kinetic energy extracted from fitting of retarding field energy analyzer versus the cluster mass. Right: Plot of the cluster mean velocity derived from the mean energy versus the cluster mass.

The overall results presented here lead to the conclusion that the cluster energy can vary significantly for a given mass of cluster based on the source conditions. This is important to know as it means the deposition energy for a given mass will change if a change is made to the source conditions and should be accounted for when attempting to control the deposition energy.

It is also worth noting that the energies measured here are quite low. The 172,394 amu cluster is composed of roughly 1,000 tantalum atoms. Its energy varies between $13.6 \mathrm{eV}$ and $20.4 \mathrm{eV}$, which is only between $0.01 \mathrm{eV}$ to $0.02 \mathrm{eV}$ per cluster atom. This is well within the soft landing region of deposition energies. This means as-deposited clusters with no bias applied to the substrate, should land intact without significant deformation or localized melting. 


\section{Effect of Mass Resolution on Kinetic Energy Distribution}

From the results of the argon flow experiment, it would be expected that a wider mass resolution will have a wider energy distribution, but same location. To test this, one mass was selected at a single flow rate and several mass resolutions and the ion current measured again as a function of retarding field potential. The results can be seen in Figure 10.16 and Table 10.7. At 100\% resolution, the error function fit does not match the data as well as with the $50 \%$ and $20 \%$. This could be because the mass distribution of the clusters which creates the broad energy distribution is not well fit by a Gaussian. Despite the deviation near the edges, the peak location and width should be close enough for comparison with the other points.
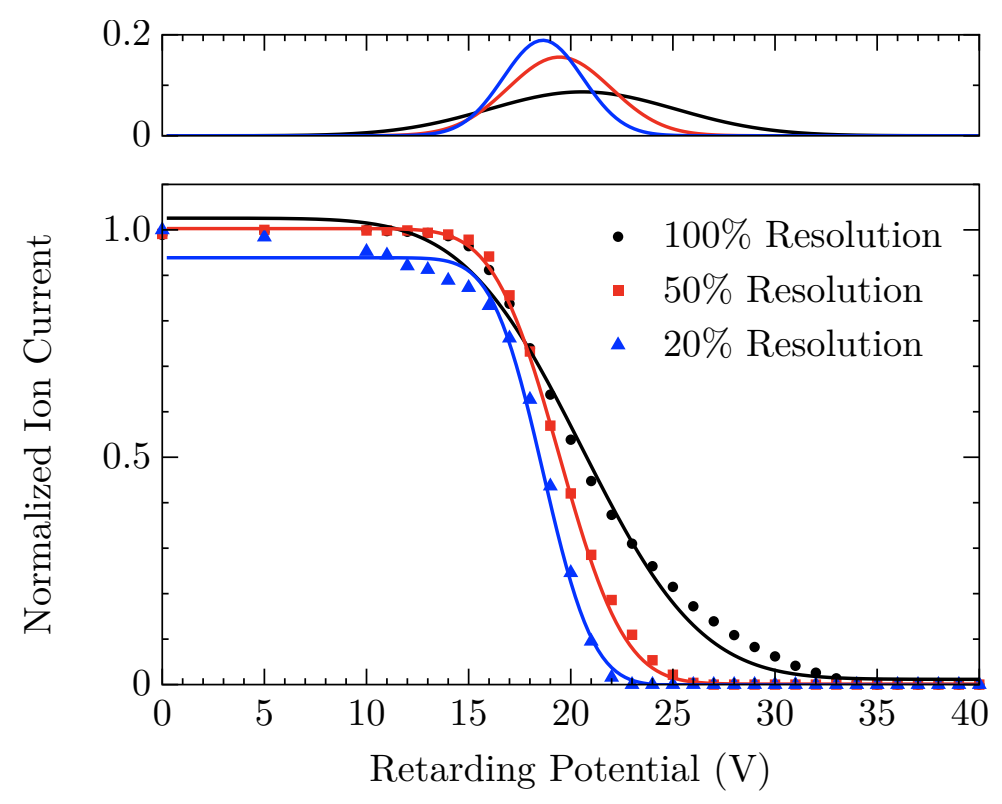

Figure 10.16: Plots of the energy distribution determined by the retarding field energy analyzer for several quadrupole filter resolutions.

The FWHM of the distribution increases substantially for the $100 \%$ resolution compared to the $20 \%$ resolution. The mean energy does shift to lower energy going from $100 \%$ to $20 \%$, but not by very much. This effect could be due to an asymmetric widening of the mass distribution - i.e. more ions with heavier masses are present in the mass distribution exiting the source, so even though lower masses could be included when the resolution increases from $20 \%$ to $100 \%$, they are simply not produced in the cluster beam.

The overall result here is as expected. If a very narrow energy distribution is desired for an application, a narrow resolution should be chosen on the quadrupole mass filter.

\section{Effect of Adding Helium to the Cluster Source}

A final experiment was performed to see what effect adding helium to the source has on the cluster energy. This is important as argon is rarely used alone in the source. A cooling 
Table 10.7: Retarding field energy analyzer extracted fit for the effect of quadrupole mass resolution on cluster energy.

\begin{tabular}{ccc}
\hline Resolution & $\begin{array}{c}\mu \\
(\mathrm{eV})\end{array}$ & $\begin{array}{c}\sigma \\
(\mathrm{eV})\end{array}$ \\
\hline $100 \%$ & 20.7 & 4.7 \\
$50 \%$ & 19.6 & 2.6 \\
$20 \%$ & 18.7 & 2.0 \\
\hline
\end{tabular}

gas such as helium is often added to promote cluster growth. It would be expected that the addition of a roughly equal amount of helium to the cluster beam would increase the beam's velocity, which would increase the kinetic energy if the model where ions gain most of their kinetic energy from the carrier gas is correct. As in the last section, one mass was chosen with a fixed argon flow at $20 \%$ mass resolution and data recorded for $0 \mathrm{sccm}$, $10 \mathrm{sccm}$ and $20 \mathrm{sccm}$ of helium added to the source.

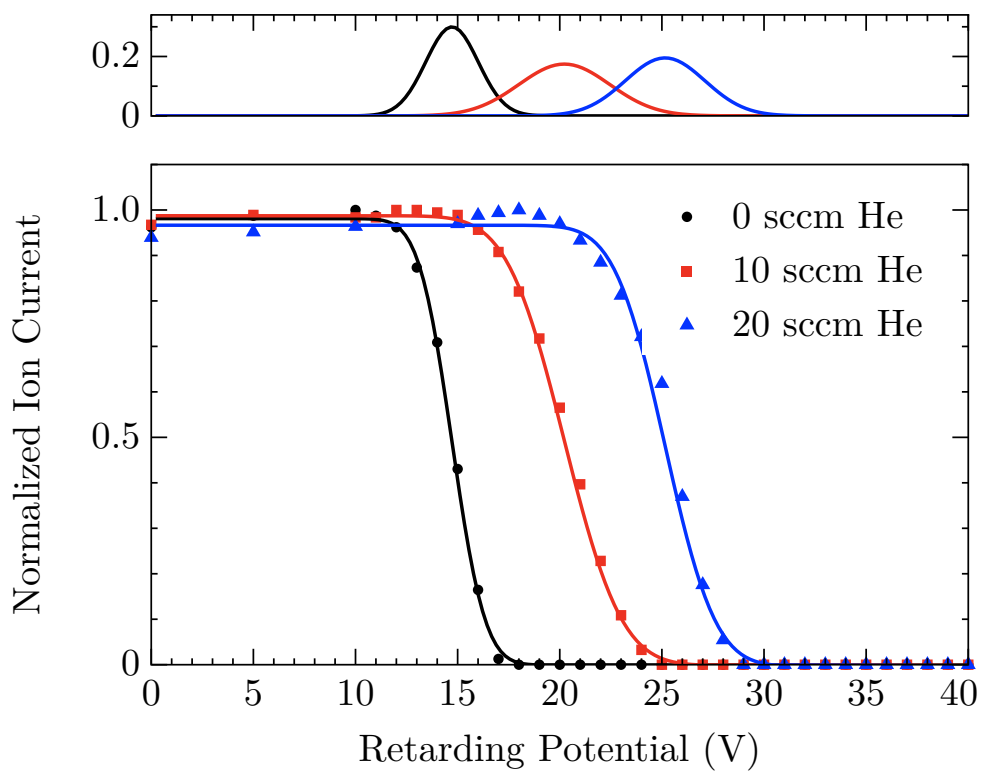

Figure 10.17: Plots of the energy distribution determined by the retarding field energy analyzer for several flow rates of helium.

The results of the mean energy and FWHM extracted from the data fits can be found in Figure 10.17 and Table 10.8. The mean energy of the cluster increases dramatically with the addition of helium, but the energy width does not have any particular trend. This is again within expectations, but an important demonstration. If controlled deposition 
Table 10.8: Retarding field energy analyzer extracted fit for the effect of helium flow rate on cluster energy.

\begin{tabular}{ccc}
\hline $\begin{array}{c}\text { He Flow } \\
(\mathrm{sccm})\end{array}$ & $\begin{array}{c}\mu \\
(\mathrm{eV})\end{array}$ & $\begin{array}{c}\sigma \\
(\mathrm{eV})\end{array}$ \\
\hline 0 & 14.8 & 1.3 \\
10 & 20.4 & 2.3 \\
20 & 25.3 & 2.0 \\
\hline
\end{tabular}

energies are required, a change to the source gas mixture can produce a substantial change to the cluster energy distribution which should be accounted for.

\subsubsection{Model for Ion Beam Velocity}

From the experimental observations, it is reasonable to postulate that the main contribution to the final cluster velocity is acceleration through collisions with a carrier gas. To examine this hypothesis, first principles are used to develop a simple model for the cluster velocity and the calculated velocities for a given mass and carrier flow combination are compared to the experimental results.

The starting point for this model assumes elastic collisions between a fully grown cluster of mass $M$, initial velocity $V_{i}$, and final velocity $V_{f}$ and the carrier gas with mass $m$, initial velocity $v_{i}$ and final velocity $v_{f}$. From conservation of momentum and energy:

$$
\begin{aligned}
M V_{i}+m v_{i} & =M V_{f}+m v_{f} \\
M V_{i}^{2}+m v_{i}^{2} & =M V_{f}^{2}+m v_{f}^{2}
\end{aligned}
$$

Solving this system of equations and finding the change in cluster velocity $\Delta V$ for a single collision leads to:

$$
\Delta V=\frac{2 m\left(v_{i}-V_{i}\right)}{(m+M)}
$$

Next I assumed the number of collisions, $N$, scales proportional to the pressure of the carrier gas, $p$, and the cross section of the cluster which should be proportional to $M^{2 / 3}$. A scaling constant, $A$, has been included.

$$
N=A p M^{2 / 3}
$$

For the same 10 masses and three argon gas flows shown in Figure 10.15, a cluster velocity was calculated by applying Equation 10.7 for $N$ collisions with the carrier gas 
argon with pressures of $0.175,0.226$ and 0.28 torr which correspond to the experimental pressures recorded for 15, 20 and $25 \mathrm{sccm}$ flow rates respectively. The results are shown in Figure 10.18. The filled symbols are the same experimental data points shown in Figure 10.15. The open symbols are the velocities calculated from this model with the scaling factor $A=4.7$, initial argon velocity $v_{i}=178 \mathrm{~m} \mathrm{~s}^{-1}$ and initial cluster velocity $V_{i}=0$.

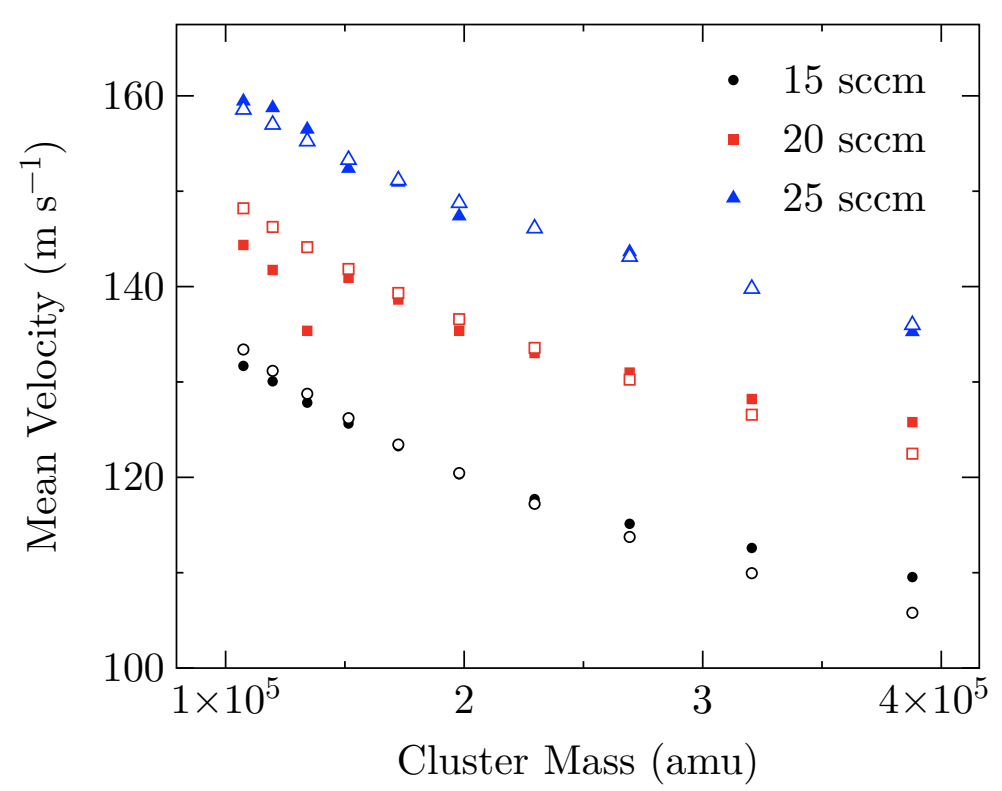

Figure 10.18: Plot comparing the calculated velocities from a simple collision model versus the experimental velocities previously shown in Figure 10.15. The filled symbols represent the experimental data and the open symbols represent the velocity calculated from the model.

As can be seen in the figure, the model follows the trend in the data extremely well. This supports the postulate that the cluster's velocity is mainly due to collisions with the argon carrier gas. This model is presented to show a reasonable mechanism for the observed experimental results and not as a method to perform $a b$ initio calculations of cluster velocities. In particular, the values for the parameters $A$ and $v_{i}$ were selected based on their fit to the data and are essentially just fitting parameters. These values are within a reasonable range, but were not based on a direct measurement of a characteristic of the system.

\subsubsection{Considerations for Controlling Ion Energy}

The results in this section show that the native kinetic energy of nanoparticles produced in this instrument is well within the soft-landing energy regime. Although the gas flow in the source is shown to influence the energy of the beam, the overall energy per cluster atom is quite low, even for the higher gas flows. This is advantageous for most applications where maintaining the structure of the nanoparticle and support surface is a requirement. 
However, there may be some applications where modification of the energy is required. If an even lower impact energy for landing the cluster on the support is desired, then a small retarding field must be applied to the substrate holder. However, the energy distribution of the cluster beam must be kept in mind as any changes to the gas flow or mass being deposited will shift the peak of the energy distribution, requiring a different retarding bias for optimal deposition.

There may also be applications where a hard impact with the surface is useful, for example to "pin" the clusters on the support surface. In this case, an attractive bias should be applied to accelerate the clusters to an energy of between 1 and $10 \mathrm{eV}$ per atom. Higher energy than $10 \mathrm{eV}$ per atom will likely result in significant damage to the surface and cluster, though this may also have its uses for producing a strongly adhered thin film of metal on the surface.

\subsection{Microscopy of Surface Deposited Size Selected Clusters}

The ultimate goal of this instrument is to prepare size selected nanoparticle samples on a variety of support substrates in a controlled process. The final characterization step to provide proof of functionality of the instrument is an examination of the clusters on support surfaces by microscopy techniques. This will allow the cluster size distribution and density on the surface to be determined. Other information can be obtained as well regarding the degree of aggregation of the clusters after deposition, thermal stability, and composition of the nanoparticles.

Two types of microscopy are used to analyze the nanoparticles after deposition - Atomic Force Microscopy (AFM) and Transmission Electron Microscopy (TEM). Each technique has a different set of benefits and challenges, and together, they provide a broad overview of the characteristics of the supported nanoparticles.

\subsubsection{Atomic Force Microscopy}

\section{Surface Preparation and Deposition}

Proper choice and preparation of substrate is important to achieving good quality AFM images. The best substrates are ones which are atomically flat and therefore the surface topography has no influence on the height measurement of particles. However, the best quality microscopy surfaces may not be the most interesting for other experiments such as catalysis. Several materials have been used as substrates for deposition, but here I will present results only for silicon (Plano), muscovite mica (Plano), and strontium titanate (MTI Corp).

Silicon is a common substrate for nanotechnology applications, is relatively inexpensive, and can be polished to near atomic flatness. Muscovite has fewer real world applications, but can be easily cleaved on its basal plane, producing a completely atomically clean 
and flat surface which is perfect for AFM analysis. Strontium titanate is another material which can be polished to near atomic flatness and may have practical applications in device fabrication.

For $\mathrm{Si}$ and $\mathrm{SrTiO}_{3}$, the substrates are highly polished on one side. The fresh substrate was cleaned by washing with acetone, followed by methanol, and then dried using compressed air. Mica is able to be cleaved by wedging a sharp knife between layers and prying them apart. For the mica substrates, an alcohol cleaned knife was used to cleave and the freshly cleaved surface was used as-is with no further cleaning or modification.

Once prepared, the substrate was immediately affixed to the sample holder and inserted into the system's load-lock in order to minimize the amount of time the clean substrate could interact with atmosphere.

Deposition was carried out with the substrate approximately $3 \mathrm{~cm}$ from the center of the surface chamber (and the center point of the nanoparticle beam) and the size selected ion beam deflected onto the substrate with an applied voltage to plates at the entrance of the surface chamber. This allows only the size selected beam to be deposited, avoiding any neutral nanoparticles which would be present in the center beam. Depositions are typically carried out for 10 to 20 minutes with measured ion currents between 10 and $50 \mathrm{pA}$.

In all experiments presented here, tantalum was used as the deposition material. This is mainly due to the experience gained with using tantalum in magnetron sputtering from the previous characterization and not for some specific property of tantalum. However, tantalum is a high melting point material which may give it better stability when exposed to high temperature environments.

After deposition, the substrate is left in vacuum until just prior to the microscopy. This is to minimize the time the particles have to interact with contaminants after leaving the vacuum system. Exposure to atmosphere is, unfortunately, unavoidable for doing microscopy analysis currently. An in-situ STM/AFM instrument would be an interesting future addition to the capabilities of the surface chamber. It is likely that the exposure to atmosphere leads to the oxidation of the particles on the surface. Composition analysis and quantifying the degree of oxidation has been attempted, however there is not yet a satisfactory answer to the degree of oxidation.

\section{Data Acquisition and Image Processing}

All AFM data presented in this section was acquired on an Agilent Technologies 5600LS Scanning Probe Microscope operated in intermittent contact mode or "tapping mode". The tips used were model TAP300-G from the company Budget Sensors.

The data was processed using the freeware microscopy program Gwyddion 2.29 [16]. All images were processed using the same series of steps. First, a second order polynomial background was subtracted in both $x$ and $y$ dimensions to account for drift in the piezoelectric drives during acquisition. Then the image was line leveled which sets the median height of each line equal to each other. A small $2 \times 2$ median filter was applied to the image to reduce random noise from significantly contributing to the particle heights. Finally, if the median surface height of an image is not zero, the image height scale was shifted to set 
the median surface height to zero. The reasoning for some of these processing steps will be clear following an explanation of the histogram process. In general, the number of steps which actually modifies the data was kept to a minimum and overly destructive processing was avoided.

To extract the height information for the particles, a simple thresholding method is used. A threshold height can be set, and any regions of the image with height above the threshold are masked. By setting the threshold above any surface defects, but below the maximum height of the smallest nanoparticle, all nanoparticles are marked by the threshold mask. Gwyddion then can automatically output a list of the maximum height of each discrete particle in the image. Note that if two (or more) particles are touching with no break in the mask, they will be counted as one individual particle. The median filter was applied to the image because this counting method will select the single highest pixel in the particle, even if it is just an anomalous noise pixel. By applying the median filter, it is less likely that the particle height will be affected by a single pixel outlier. Furthermore, the height is output relative to the zero height, so the re-dimensioning to make the surface height zero improves the consistency of the height values between different images.

Finally, the output list of heights is histogrammed into $0.25 \mathrm{~nm}$ bins. For most surfaces, several images are acquired. In this case, the height results of multiple images are combined into a single size distribution histogram for that surface.

\section{Evaluation of Spherical Particle Approximation}

There are several reasons why using a spherical particle approximation as described in Chapter 8.2.6 may not be reliable in this type of experiment. First, it relies on the density of the bulk material to determine the volume. This density may not be the same for nanoparticles. Second, the particle will not necessarily have a spherical shape on the surface, even if it forms as a sphere in the cluster source. Impact with the surface may create a flattening effect, or the particle could embed into the surface. While the energy analyzer results show the energy to be low enough that this is unlikely, it may still occur due to the force of interaction between the particle and substrate surface. Even if the particle lands intact, the strength of the interaction with the surface may also induce structural changes. These deviations would be expected to give a particle which is flattened compared to the approximated sphere. This may lead to particles observed by AFM having smaller heights than the approximation estimates and larger diameters when the cross-section is observed by TEM.

Flattening is not the only possible deviation from the spherical approximation. As mentioned previously, exposure to atmosphere is currently unavoidable. This can lead to particles oxidizing which can cause them to grow larger than the size expected from this approximation.

The height expected from a spherical particle approximation for a nanoparticle of a given mass is shown on the histograms in the figures in this section with a red dashed line. Due to the many possible flaws of this approximation, this is just meant to serve as a guide to the size expected from a simple formula and not as the absolute measure of the success of 
Chapter 10 Characterization and Preliminary Results

the particle size selection.

\section{Challenges of AFM}

While there is no doubt that AFM is a powerful method for producing surface topography on a small scale and that it can have a very high resolution when observing heights, there may be some doubt as to the applicability of the method to measuring nanoparticle sizes in the very small $<10 \mathrm{~nm}$ range. Some reports in literature conclude that capillary forces between the AFM tip and the surface produce a change in the oscillation frequency of the cantilever which then changes when the tip interacts with the particle [31, 36, 38, 39, 54, 59]. This can result in a change in the measured height which is not based on the topography, but on the interaction force of the tip with the surface and particle. Personal observations show that the height of nanoparticles measured on the same region of a substrate can vary by more than $1 \mathrm{~nm}$, just by changing the tip. As there currently does not seem to be a well established method for accounting for this effect, every effort was made to reduce its impact on the results. As much as possible, the same AFM tip was used when measuring a series of results, to avoid a change in the capillary force due to a change in the tip's surface area. The user adjustable parameters were kept constant as much as possible while still obtaining high quality data.

While there may be some doubt to the absolute size measured here, there are several characteristics of the particles which can be observed including surface coverage, aggregation properties and relative width of the size distribution. To more accurately observe the particle size and structure, transmission electron microscopy was also performed and those results will be presented in a later section.

\section{Comparison of Tantalum Nanoparticles Deposited on Different Substrates}

First, I will present the results of approximately the same size particles deposited on the substrates $\mathrm{Si}, \mathrm{SrTiO}_{3}$, and mica. Figure 10.19 shows the Si substrate with 171,188 amu tantalum particles (25\% quadrupole mass resolution). This mass converts to $3.2 \mathrm{~nm}$ in the spherical particle approximation. As the histogram of the particle heights in the figure shows, the peak of the height distribution correlates extremely well with the expectation, though the width of the distribution spans about $2 \mathrm{~nm}$. The surface is quite rough, which may lead to a broadening of the distribution. It can also be seen that the surface coverage is quite reasonable, if not a bit too dense. Ideally, the majority of particles will not be close to touching a near neighbor in order to minimize the chance of sintering or aggregation, especially when used in real experimental conditions.

Figure 10.20 shows the results of depositing 237,000 amu Ta particles on $\mathrm{SrTiO}_{3}(25 \%$ quadrupole mass resolution). This mass corresponds to a size of $3.55 \mathrm{~nm}$, and the histogram of heights is very close to this point. The surface roughness appears better than the $\mathrm{Si}$ substrate, but the distribution is still about $2 \mathrm{~nm}$ wide. The density of particles is even higher than on the Si substrate, but even the closely packed particles still appear to be discrete particles. There does not appear to be a high amount of sintering under ambient 

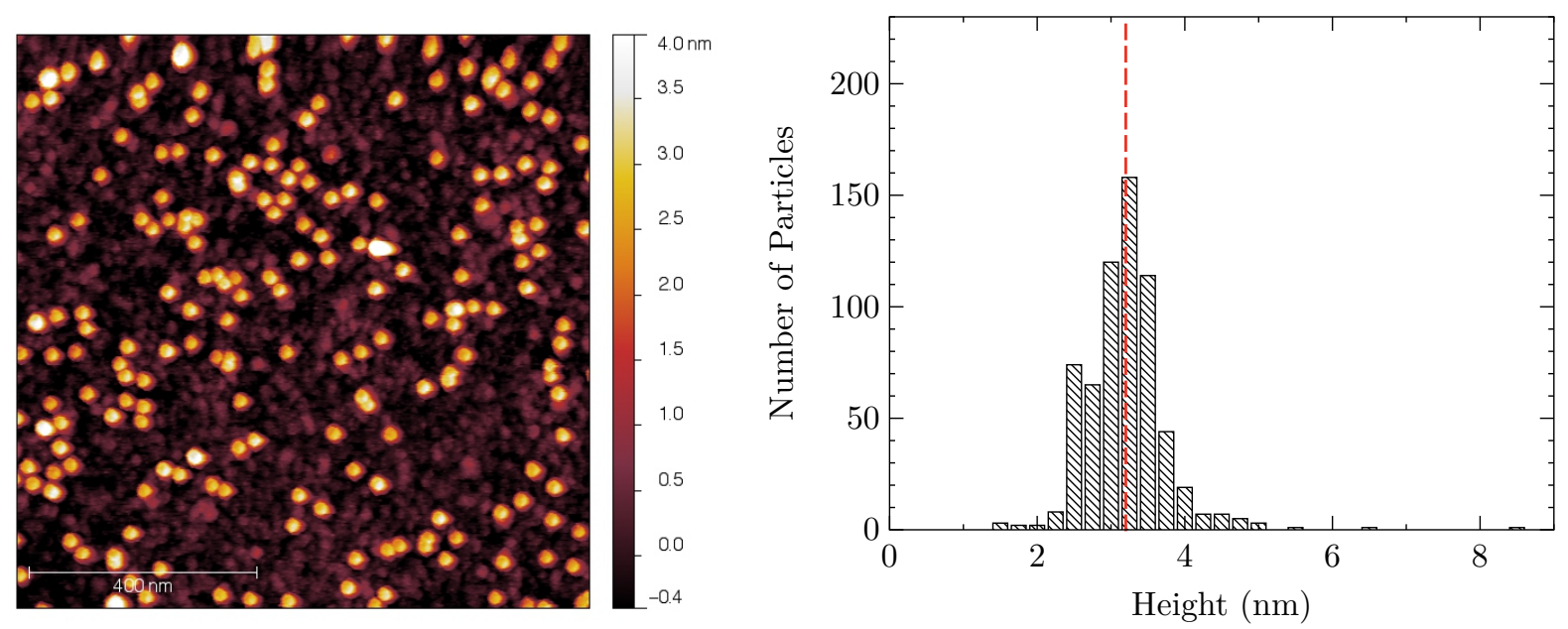

Figure 10.19: Left: Tapping mode AFM image of $171,188 \mathrm{amu}$ tantalum nanoparticles deposited on silica substrate. Right: Histogram of nanoparticle sizes extracted from the AFM images of this substrate. The dashed red line is the size expected from this mass in a spherical particle approximation.

conditions.

Figure 10.21 shows a mica substrate deposited with the exact same conditions as the Si substrate $(171,188 \mathrm{amu}$ at $25 \%$ mass resolution). The quality of the mica surface is clearly superior and it is essentially atomically flat. The reason it is a common AFM substrate is readily apparent. For some reason, despite similar deposition ion currents, the density of particles on the surface is much lower. The size distribution is the furthest from expectation of all three substrates, being smaller than the approximated size by almost $1 \mathrm{~nm}$. However, the width of the distribution is also only about $1 \mathrm{~nm}$, which is an improvement over the other two.

Comparing the three surfaces, the mica seems superior for obtain true size distributions which are unaffected by surface roughness. The particle size is quite a bit smaller than expected, but this could be due to a stronger interaction between the particle and substrate which leads to a flattening or an artifact of tip surface interaction as previously described. Overall, the tantalum particles seem quite stable under atmospheric conditions on all three substrates, with little observation of large aggregates on any of the substrates.

\section{Effect of Mass on Particle Size}

In the previous section, despite being nearly the same expected size, all three substrate surfaces showed different size distributions. To prove the size of the particles correlates with mass, a much lower mass particle was deposited on the $\mathrm{Si}$ and $\mathrm{SrTiO}_{3}$ substrates to observe the size difference.

Tantalum particles with a mass of 49,129 amu (2.1 nm expected size) and 25\% mass resolution as deposited on Si is shown in Figure 10.22. Compared to Figure 10.19, the size 

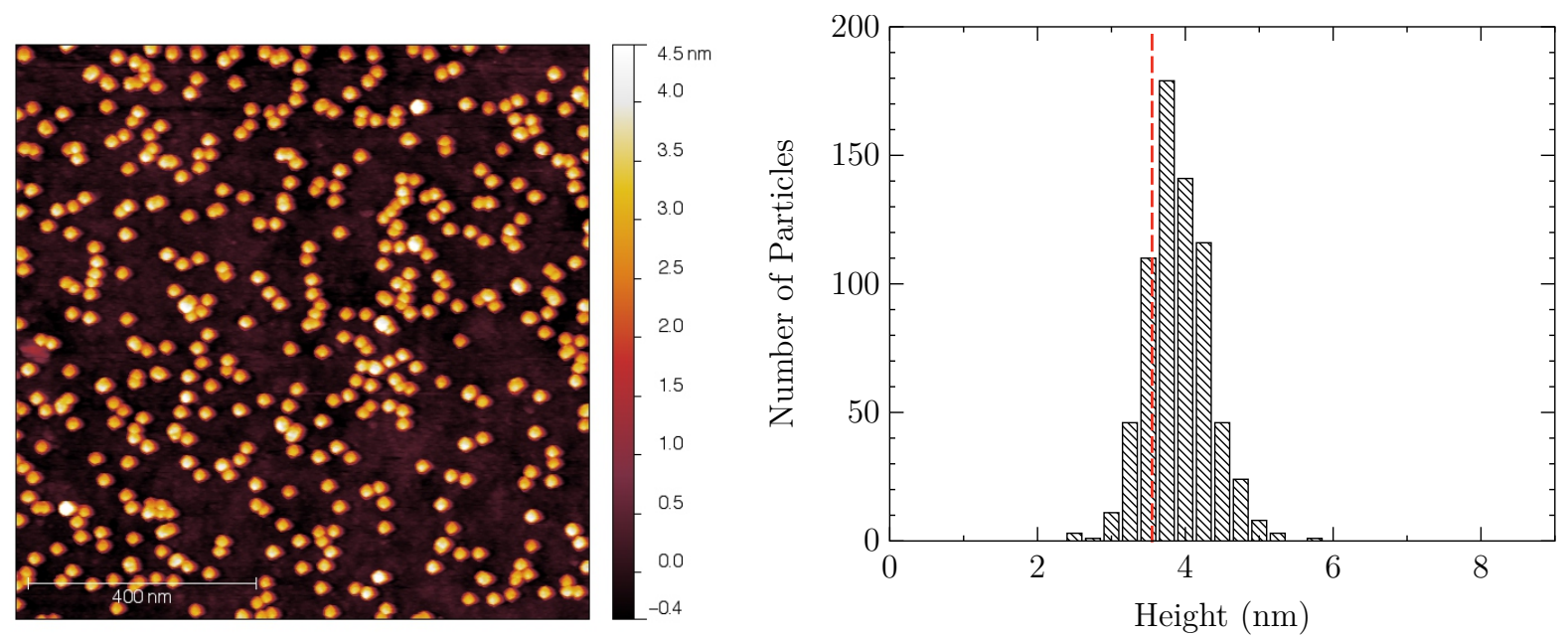

Figure 10.20: Left: Tapping mode AFM image of 237,000 amu tantalum nanoparticles deposited on $\mathrm{SrTiO}_{3}$ substrate. Right: Histogram of nanoparticle sizes extracted from the AFM images of this substrate. The dashed red line is the size expected from this mass in a spherical particle approximation.

is much smaller and again lines up exactly with the spherical particle approximation size. The surface roughness is not ideal, which may lead to a broadening of the observed height distribution.

Figure 10.23 has 61,628 amu (25\% mass resolution) Ta particles deposited on it. Compared to Figure 10.20, the size is markedly decreased. The overall size is smaller than the expectation, but this could be due to a number of factors as explained previously. The peak of the distribution is also much narrower.

Overall, these results show that the size of the particles on the surface correlate in a sensible way with the mass of the particle which is selected during the deposition process.

\section{Effect of Mass Resolution on Measured Particle Size}

To observe whether the broadness of the distributions is due to particle aggregation or sintering after deposition or just due to the width of the mass distribution in the ion beam, two depositions on mica were performed with the same selected mass at $10 \%$ resolution and $50 \%$ resolution. Mica is a good substrate for this experiment as it has no obvious surface roughness contribution to the particle size distribution.

The 10\% resolution deposition can be seen in Figure 10.24 and the $50 \%$ in Figure 10.25. The histogram in the $50 \%$ resolution deposition is clearly wider than the $10 \%$ resolution, but both are broader than expected if the mass resolution were directly converted to size resolution.

These results demonstrate that the mass resolution of the ion beam does play a role in the width of the distribution of the deposited particles, but that it may not be the only contributing factor. Separating the performance of the deposition source from the perfor- 

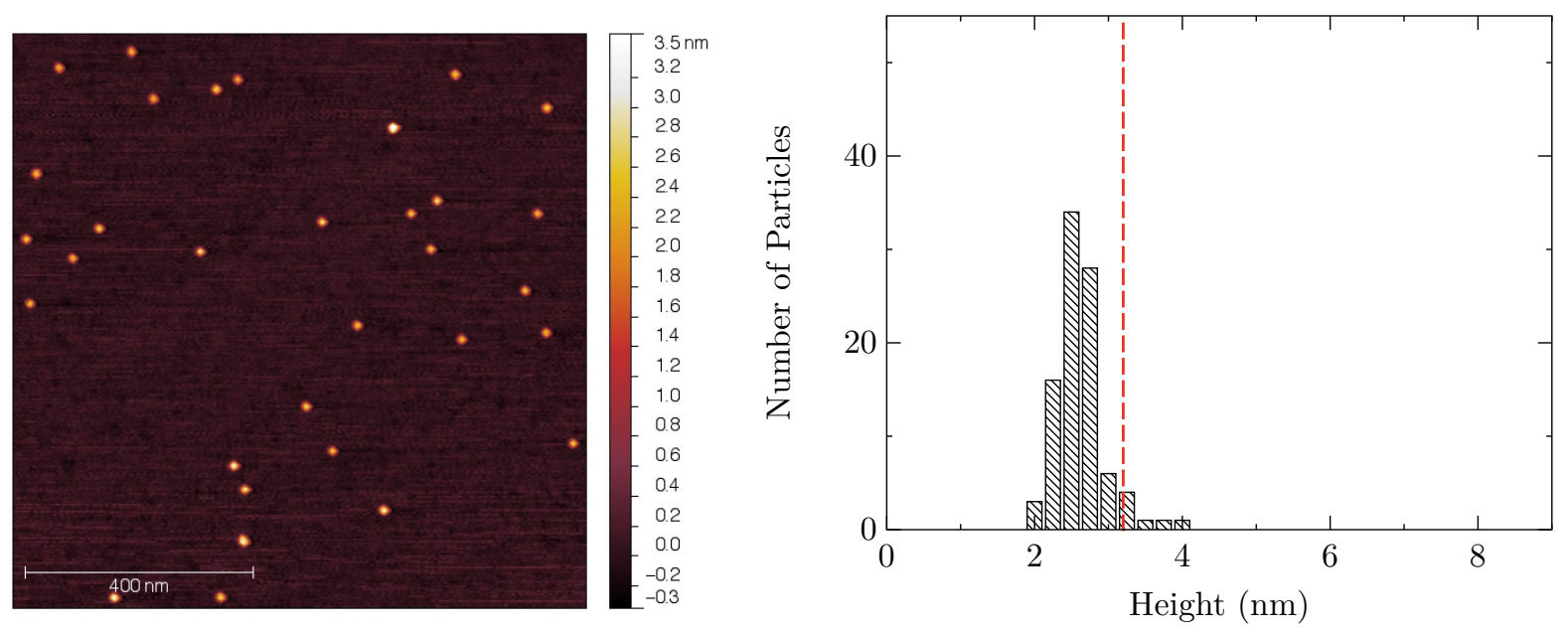

Figure 10.21: Left: Tapping mode AFM image of 171,188 amu tantalum nanoparticles deposited on muscovite mica substrate. Right: Histogram of nanoparticle sizes extracted from the AFM images of this substrate. The dashed red line is the size expected from this mass in a spherical particle approximation.

mance of the microscope is a difficult challenge. Furthermore, the necessity of exposing the particles to atmosphere could lead to oxidation processes which broaden the size distribution due to oxide layers forming. Quantifying these processes is also quite challenging and probably not possible with only AFM.

\section{Thermal Stability of Particles on Surface}

AFM was used to investigate the thermal stability of the tantalum nanoparticles deposited on a mica surface. After depositing 200,000 amu particles on the surface, a room temperature AFM scan was made. The substrate was then placed in a quartz tube furnace and heated to $200^{\circ} \mathrm{C}$ for one hour in an argon atmosphere. After cooling, another AFM scan was made. The process was repeated on the same sample to $600^{\circ} \mathrm{C}$ and $1000^{\circ} \mathrm{C}$. The mica substrate was completely destroyed by the $1000^{\circ} \mathrm{C}$ temperatures, so no data is presented for that temperature.

The three usable temperature results are shown together in Figure 10.26. The top panel is at room temperature and the distribution and density are as expected for this mass and deposition condition. The middle panel shows the surface after $200^{\circ} \mathrm{C}$ heating for one hour. The size appears to have increased by around $0.5 \mathrm{~nm}$, but overall there is not much change. The number density of clusters on the surface is the same as at room temperature, so it cannot be that several particles sintered to cause the size shift as that should result in a reduction in the number density of particles on the surface.

Perhaps even more interesting is the bottom panel, which shows the same sample after additional heating to $600^{\circ} \mathrm{C}$ for one hour. The size distribution and number density is still roughly the same. In fact, the size is smaller than in the $200^{\circ} \mathrm{C}$ scan. This could 

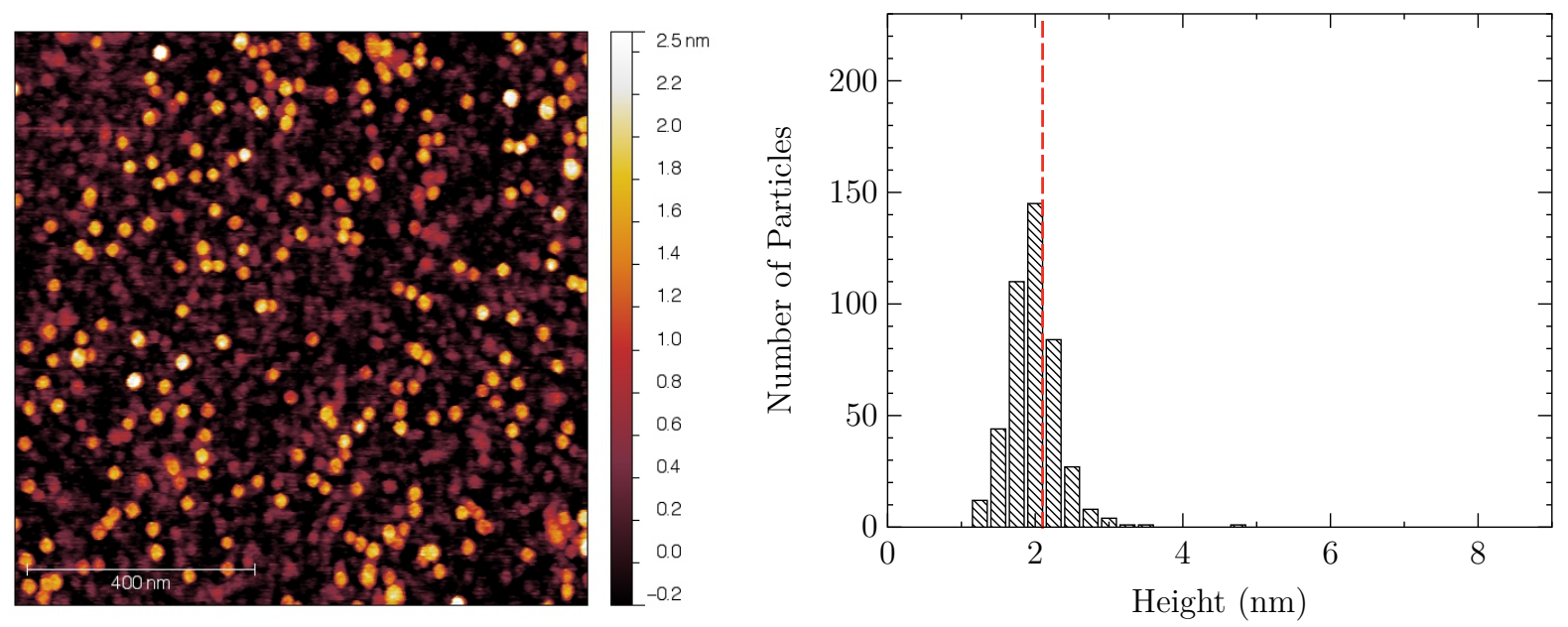

Figure 10.22: Left: Tapping mode AFM image of 49,129 amu tantalum nanoparticles deposited on silica substrate. Right: Histogram of nanoparticle sizes extracted from the AFM images of this substrate. The dashed red line is the size expected from this mass in a spherical particle approximation.

just be due to variations in the measurement conditions, but it is still interesting that the nanoparticles appear to be completely stable at this elevated temperature for long periods of time. At $600^{\circ} \mathrm{C}$ some surface defects begin to appear on the mica, but they are quite small and should not significantly impact the size distribution. By $1000^{\circ} \mathrm{C}$, they become large blisters which make a quantitative measurement impossible.

This result is encouraging for future experimental studies. Industrially relevant catalytic processes are often done below $600^{\circ} \mathrm{C}$, so this result implies these particles may be stable in a catalytically interesting temperature range. It may not be too surprising, since Ta has a high melting point, but there have not been many studies of Ta nanoparticles in this size range. Nanoparticles which are resistant to sintering at this temperature should have more research done to see if they can catalytically activate any important processes. 

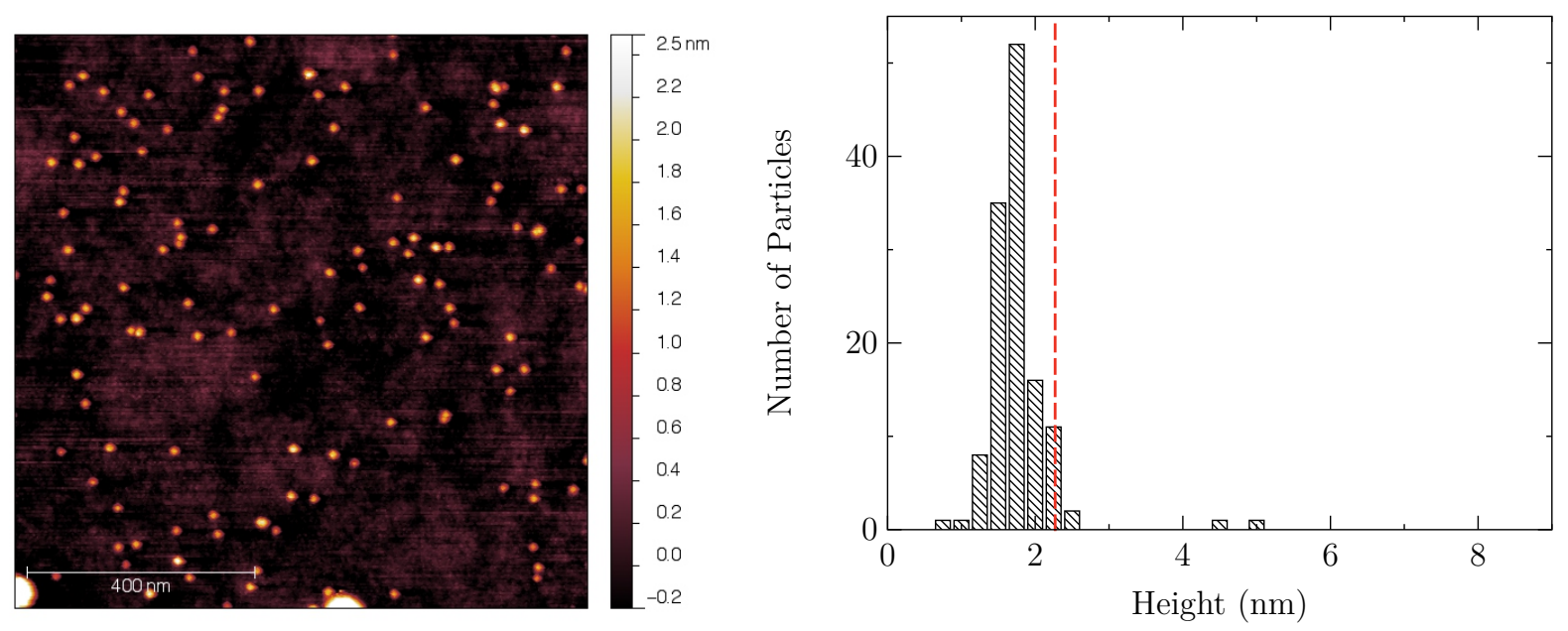

Figure 10.23: Left: Tapping mode AFM image of 61,628 amu tantalum nanoparticles deposited on $\mathrm{SrTiO}_{3}$ substrate. Right: Histogram of nanoparticle sizes extracted from the AFM images of this substrate. The dashed red line is the size expected from this mass in a spherical particle approximation.
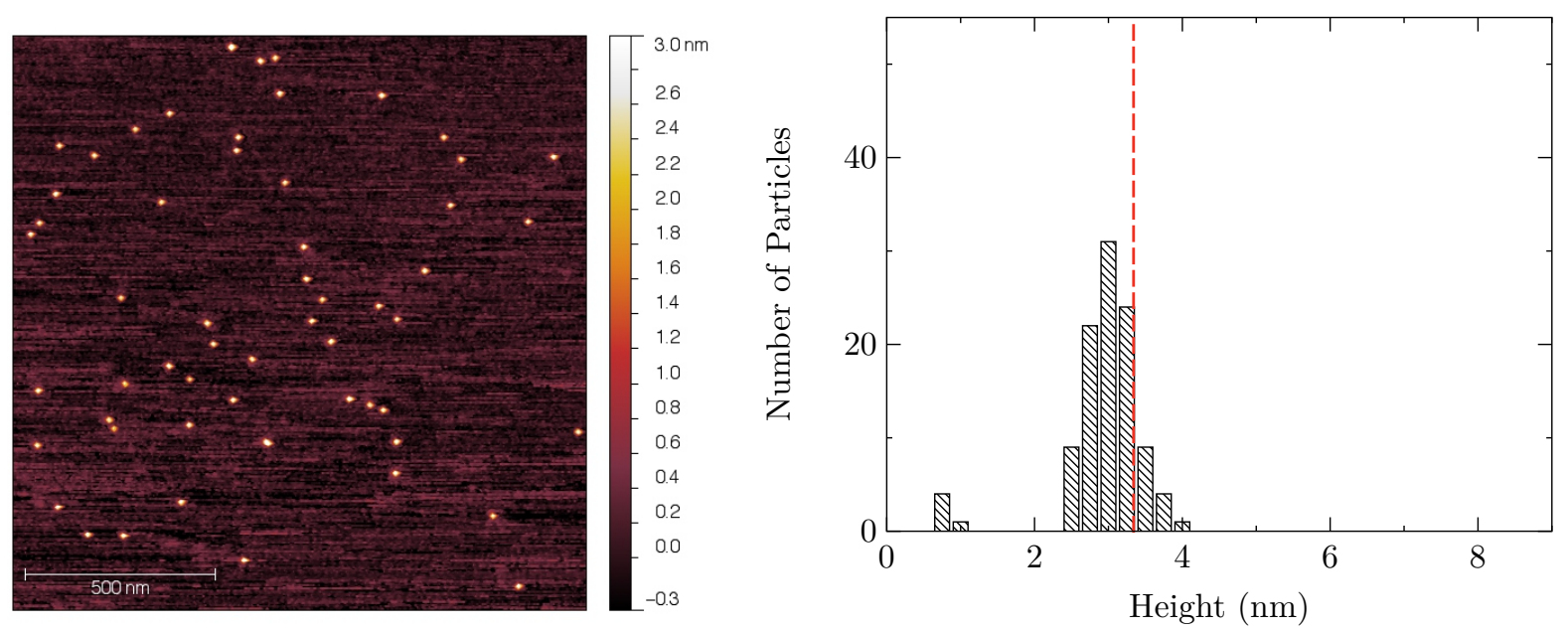

Figure 10.24: Left: Tapping mode AFM image of 196,000 amu tantalum nanoparticles with a $10 \%$ quadrupole mass resolution deposited on muscovite mica substrate. Right: Histogram of nanoparticle sizes extracted from the AFM images of this substrate. The dashed red line is the size expected from this mass in a spherical particle approximation. 
Chapter 10 Characterization and Preliminary Results
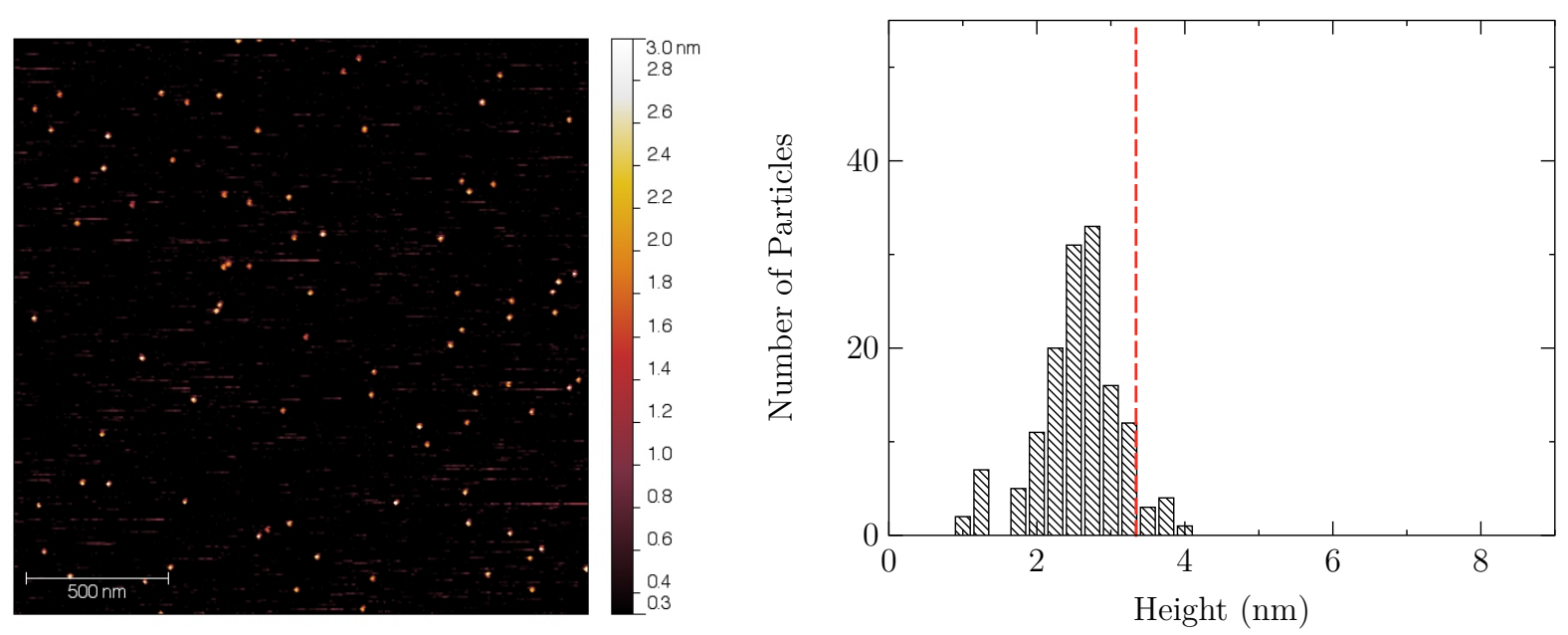

Figure 10.25: Left: Tapping mode AFM image of 196,000 amu tantalum nanoparticles with a $50 \%$ quadrupole mass resolution deposited on muscovite mica substrate. Right: Histogram of nanoparticle sizes extracted from the AFM images of this substrate. The dashed red line is the size expected from this mass in a spherical particle approximation. 

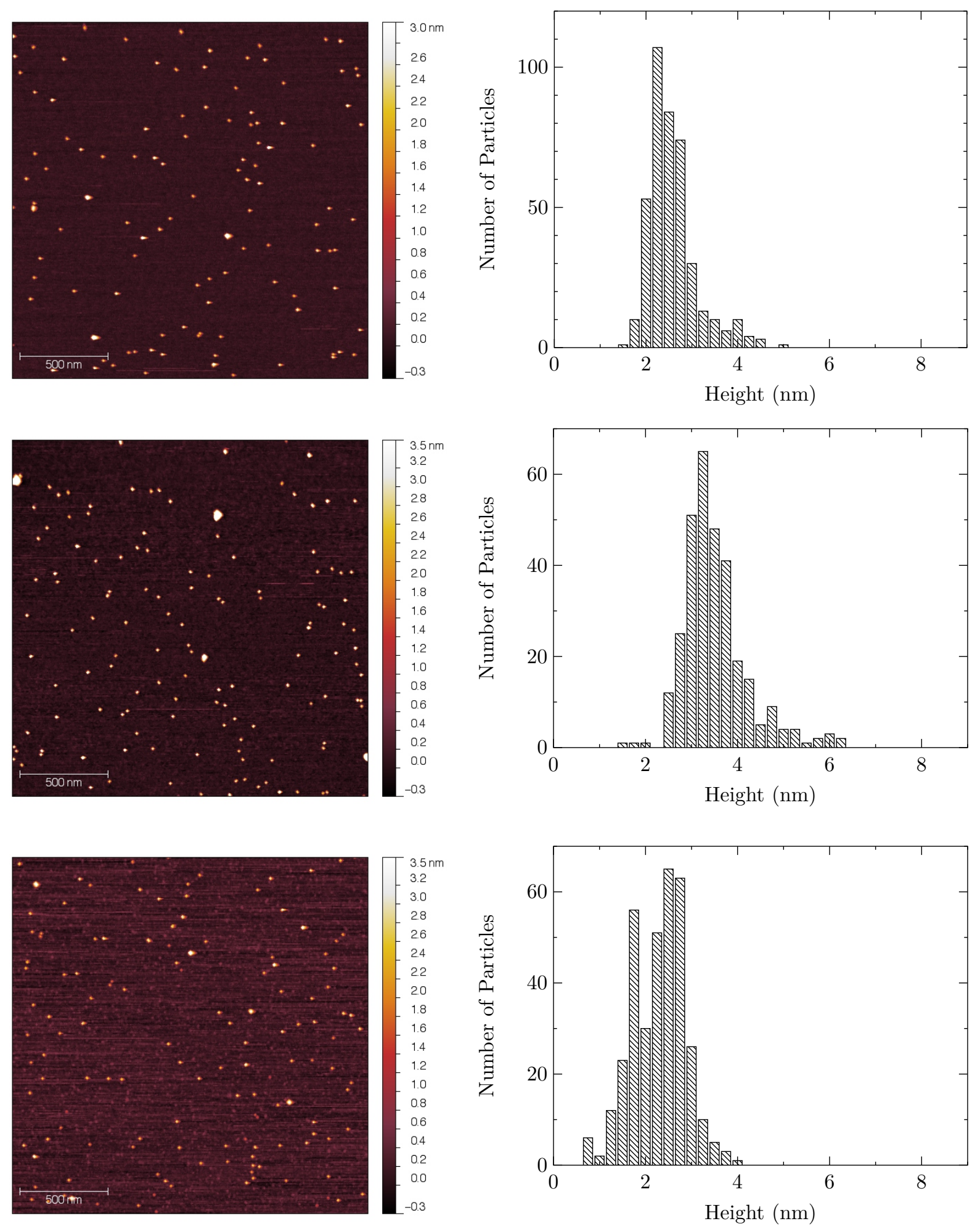

Figure 10.26: Top: As-deposited sample at room temperature. Middle: Same sample after heating to $200^{\circ} \mathrm{C}$ for one hour. Bottom: Same sample after additionally heating to $600^{\circ} \mathrm{C}$ for one hour. 


\subsubsection{Transmission Electron Microscopy}

As a complement to the AFM studies, Transmission Electron Microscopy (TEM) was performed on similar mass tantalum nanoparticles as observed in the AFM. The TEM has additional advantages in allowing for structural determinations at high resolution and elemental analysis using Energy Dispersive X-Ray Spectroscopy (EDX).

\section{Substrate Information}

TEM is far more restrictive on what substrates can be used than AFM. Essentially, only extremely thin films on mesh grids can be used. For all the images acquired in this section, a so-called "lacey" carbon coated grid was used. Copper was used as the grid material in most images. Only once was a gold grid used for improved EDX results. A graphene coated substrate was also used once as it provides the thinnest possible support material which should allow for the best possible contrast of the nanoparticles. Due to the extremely delicate nature of the support films, no cleaning was performed and the grids were used as-acquired.

\section{Instrument Information}

The instrument used to acquire TEM images in this section is a Philips CM200-FEGUT transmission electron microscope. It features a field-emission gun (FEG) and high resolution objective lens (Ultra Twin). The theoretical spacial resolution of this instrument is as low as $0.187 \mathrm{~nm}$. It is equipped with an Oxford ISIS Energy Dispersive X-Ray Spectrometer for chemical composition analysis. All images were acquired with an electron beam energy of $200 \mathrm{keV}$.

\section{Effect of Mass on Size Observed in TEM}

The first nanoparticles observed in the TEM were mass 200,000 amu (20\% mass resolution) deposited on a lacey carbon coated copper TEM grid. This is the same mass used in a number of the AFM studies in the previous section and should provide complimentary information to the results obtained there. A representative image of the particles can be seen in Figure 10.27. As observed in AFM, the particles appear randomly dispersed at high coverage, with no sign of significant aggregation on this substrate.

To process the image and obtain a statistical histogram of the particle sizes, the freeware program ImageJ was used [47]. Due to the significant interference from the background of the carbon film, more image processing needed to be done to obtain reliable results than in the AFM images. First a bandpass filter and $3 \times 3$ median filter were applied to suppress the noise. Then the contrast of the particles against the background was improved by a contrast stretching algorithm with $2.5 \%$ of the pixels being saturated. As with the AFM, a threshold was chosen which masked mostly particles with as little contribution from background pixels as possible. There was no threshold possible which completely avoided anomalous background noise, so the analysis focused only on particles larger than $6 \mathrm{~nm}^{2}$. 


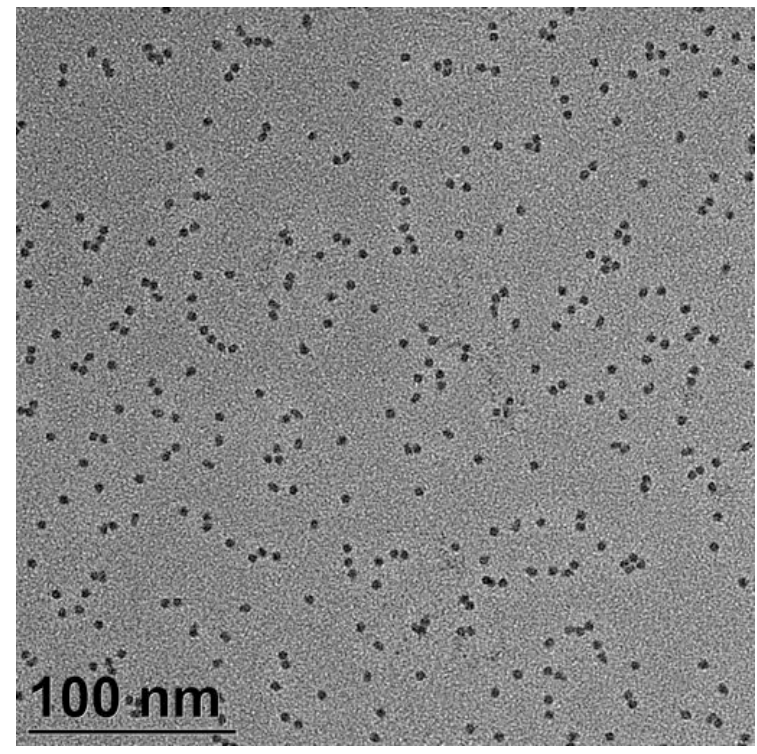

Figure 10.27: Raw transmission electron microscope image of 200,000 amu tantalum nanoparticles deposited on a lacey carbon coated copper grid.

To avoid any large aggregates, the maximum size was limited to $30 \mathrm{~nm}^{2}$. These limits are well outside the range of observed real particles at this mass. From this threshold a binary image was created with black particles on a white background. Finally, ImageJ can output the area of each discrete particle marked from the thresholding. To avoid counting any particles whose shape could not be well defined by a circle, only those particles with circularity above 0.7 were analyzed.

From this list of areas, the particle diameter could be determined by assuming a circular shape and the formula for the area of a circle. The resulting histogram is shown in Figure 10.28. The expected size from a spherical approximation of the mass is shown as a dashed line. The particle size is larger than expected, but narrower than seen in the AFM results. The majority of particles are within $0.5 \mathrm{~nm}$ of each other.

This size and resolution are in-line with the results of the AFM, but these should be more accurate. There are less factors which may effect the observed size in TEM than as described in AFM.

Next, 800,000 amu Ta nanoparticles were deposited on an identical grid. A representative TEM image can be seen in Figure 10.29. The results here are quite different from the 200,000 amu particles. First, it appears there is a high degree of aggregation and sintering at this mass. Almost all of the particles are in direct contact with at least one other particle and in many cases, it is hard to distinguish exactly how many particles are in an aggregate. This makes it impossible to apply a thresholding filter, which makes the size analysis difficult. Even manual counting is challenging due to the asymmetric and oval shapes which makes consistency in measurement hard. It is clear to the eye that the particles are quite a bit larger than those seen in Figure 10.27. For manually measuring particles, most of them have a width in the narrower dimension between 5 and $6 \mathrm{~nm}$. The 


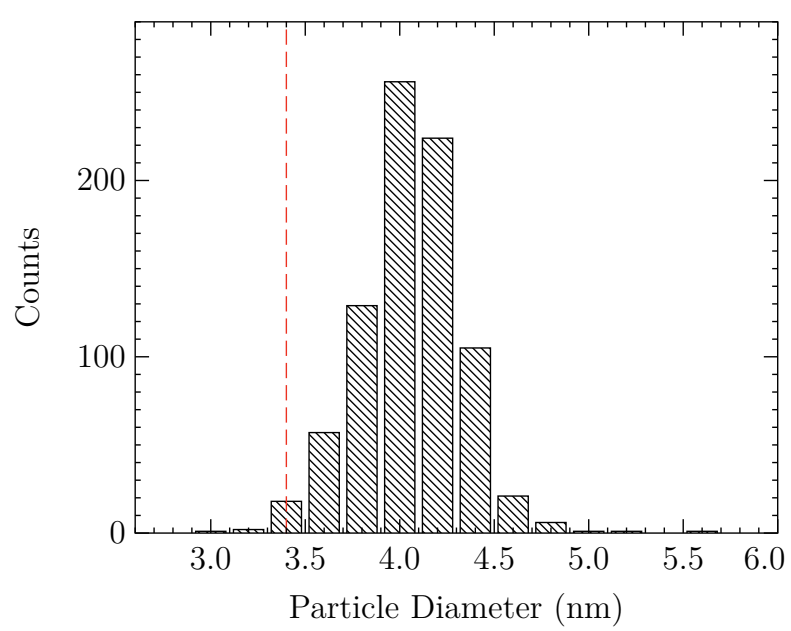

Figure 10.28: Histogram of 200,000 amu tantalum nanoparticle sizes extracted from TEM images. The dashed red line indicates the expected size from a spherical particle approximation.

expected size from the spherical particle approximation is $5.33 \mathrm{~nm}$.

It is unclear why the larger particles seem more prone to aggregation. The surface coverage is quite high, but even closely spaced particles of 200,000 amu mass did not seem prone to sintering.

As larger particles had aggregation problems, a much lower mass was deposited next. Figure 10.30 is an image taken of 50,000 amu Ta nanoparticles. This mass has an entire different problem. The particles have much too low of a contrast on the carbon film. It is almost impossible to clearly distinguish the boundary of the particles, although it is clear to the eye they are substantially smaller than those in the previous two images. Once again, the thresholding is not possible and the manual counting is unreliable as well since no clear boundary from particle to background can be determined. My best estimate from manual measurements for this size is between 2.5 and $3 \mathrm{~nm}$. The expected size from the spherical particle approximation is $2.11 \mathrm{~nm}$.

The aggregation seems very low. The particles are well dispersed and there are clearly no very large particles which would produce better contrast. So from these results, it appears that the low mass Ta nanoparticles undergo less aggregation and sintering than high mass Ta nanoparticles when deposited on carbon films. This is an interesting result which could be explored more in future experiments.

With the high degree of aggregation in large particles and the very low contrast in the smallest particles, one more mass was examined - 300,000 amu. This mass is similar to the 200,000 amu which produced the only statistically analyzable set of data, but should be about $0.4 \mathrm{~nm}$ larger. Figure 10.31 shows that the particles do have usable contrast like Figure 10.27 and a low degree of aggregation. The histogram of particle diameters was extracted with the same method as the 200,000 amu particles and the results are shown in Figure 10.32. 


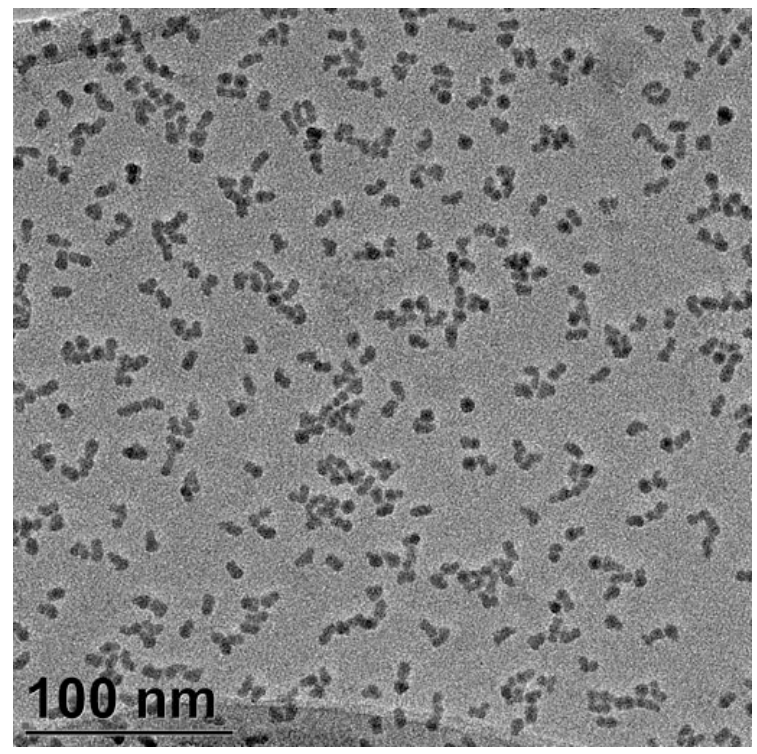

Figure 10.29: Raw transmission electron microscope image of 800,000 amu tantalum nanoparticles deposited on a lacey carbon coated copper grid.

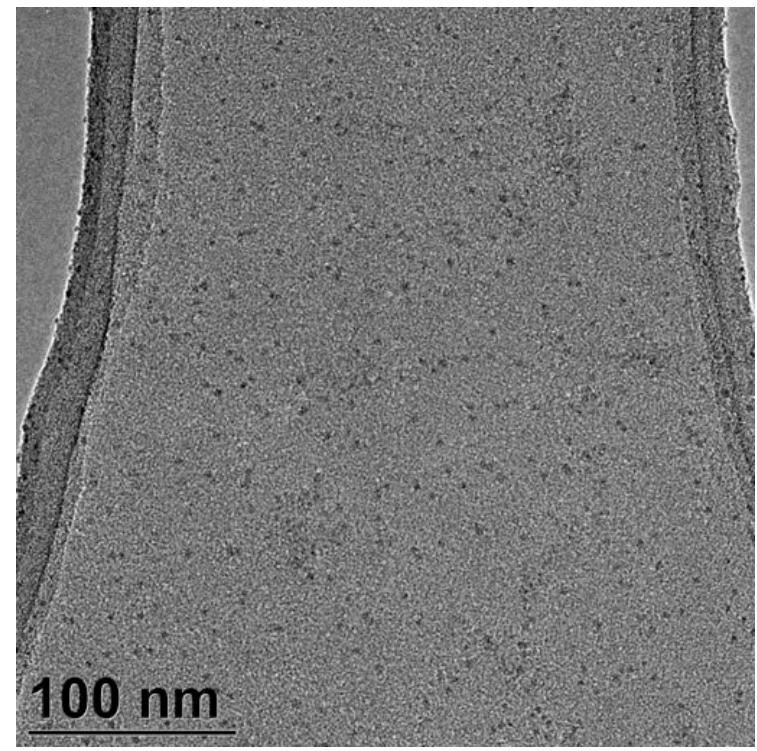

Figure 10.30: Raw transmission electron microscope image of 50,000 amu tantalum nanoparticles deposited on a lacey carbon coated copper grid. 


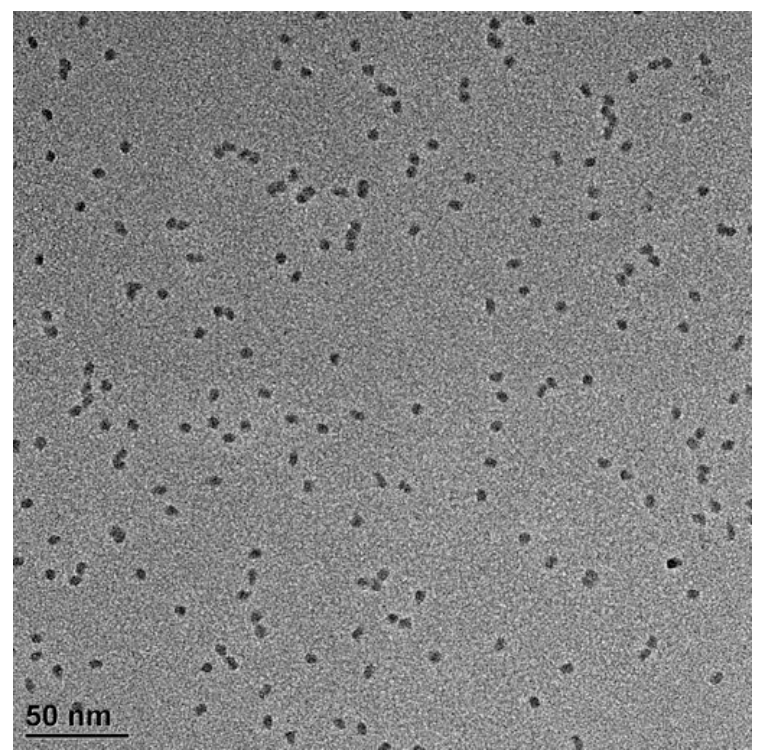

Figure 10.31: Raw transmission electron microscope image of 300,000 amu tantalum nanoparticles deposited on a lacey carbon coated gold grid.

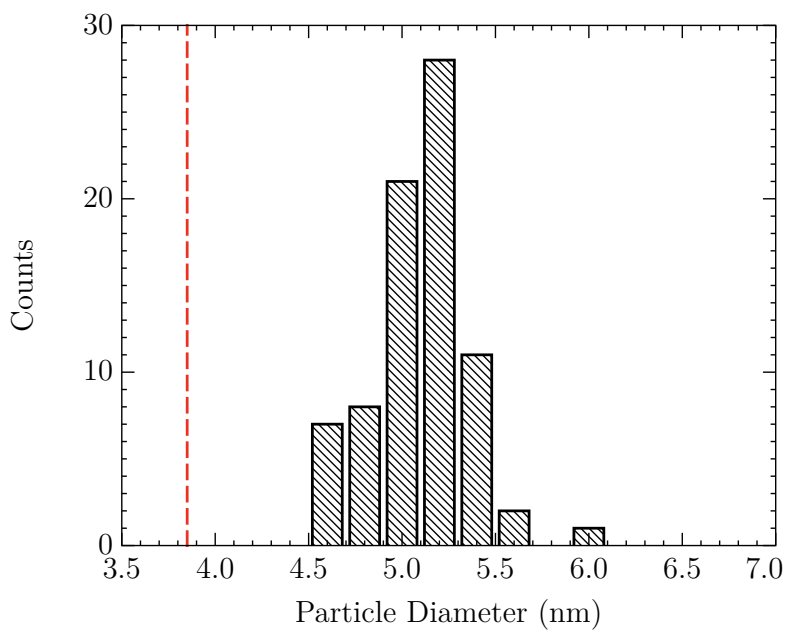

Figure 10.32: Histogram of 300,000 amu tantalum nanoparticle sizes extracted from TEM images. The dashed red line indicates the expected size from a spherical particle approximation. 
The size of the particles did increase from 200,000 amu to 300,000 amu, however, they are substantially larger than expected - with the peak shifting not $0.4 \mathrm{~nm}$ but about $1.2 \mathrm{~nm}$. Once again, this could just be due to flattening of the particles on the surface. It also can be indicative of oxidation of the particles causing them to increase in size far beyond the prediction. To investigate the role oxygen may play, EDX analysis was performed on these 300,000 amu particles.

\section{Energy Dispersive X-Ray Spectroscopy}

Energy dispersive X-ray spectroscopy (EDX) is a common analytical technique available in electron microscopes - both scanning and transmission. The high energy electrons in the microscope beam can eject an inner shell electron from an atom creating a hole. An outer shell electron then fills the hole and an X-ray photon is emitted with an energy equal to the difference between the two orbital levels. The energy difference between the orbitals is distinct for different atoms, and hence, by measuring the energy of emitted X-rays from the sample, the elemental composition can be determined. This method can be quantitative under the right conditions, as it is possible to accurately estimate the factors which affect $\mathrm{X}$-ray emission and adsorption.

EDX spectra were measured for points both on and off of tantalum nanoparticles. Representative plots can be seen in Figure 10.33. A table summarizing relevant X-ray energies by element can be found in Table 10.9. These spectra clearly show the presence of tantalum when the beam spot is focused on a particle versus empty carbon film. Carbon, oxygen and gold peaks are seen in both. Gold was the TEM grid material, and this peak is likely the result of secondary X-ray emission, not the presence of gold throughout the sample. Carbon will be present everywhere due to it being the support film. The presence of oxygen is the central question leading to performing EDX in the first place. As can be seen, there is a small oxygen peak present both on and off of the particle. There is a small increase in the peak when on the particle, but it is not enough to determine the quantity of oxygen in the sample. The increase observed here can just be due to an increase in the Bremsstrahlung for the higher atomic mass of the tantalum atoms. There is a noticeable increase in the background noise in this 0 to $2.5 \mathrm{keV}$ region of the spectrum which is indicative of Bremsstrahlung.

A line scan was also performed and the relevant spectra can be seen in Figure 10.34. For the line scan, the number of counts in a peak corresponding to a given characteristic $\mathrm{X}$-ray are plotted as a function of position while the electron beam moves along a line. Three Ta nanoparticles were present in the line scan. The Ta X-ray spectrum clearly shows when the beam crosses over the particles - though two of the particles are too close to be separately resolved. The other elements are essentially evenly distributed across the line. The number of oxygen counts is statistically meaningless and so the question of oxygen presence is still unanswered.

Unfortunately, oxygen is one of the most difficult elements to quantify using EDX. The detector efficiency for X-rays with energy below $1 \mathrm{keV}$ is extremely low. This, coupled with the observed presence of oxygen in the carbon film from the point scans, makes determining 
Table 10.9: Table of relevant X-ray energies adapted from Reference [53].

\begin{tabular}{ccc}
\hline Element & Line & $\begin{array}{c}\text { Energy } \\
(\mathrm{keV})\end{array}$ \\
\hline $\mathrm{C}$ & $\mathrm{K}_{\alpha}$ & 0.277 \\
$\mathrm{O}$ & $\mathrm{K}_{\alpha}$ & 0.525 \\
$\mathrm{Ta}$ & $\mathrm{M}_{\beta}$ & 1.760 \\
$\mathrm{Ta}$ & $\mathrm{L}_{\alpha_{1}}$ & 8.147 \\
$\mathrm{Au}$ & $\mathrm{M}_{\beta}$ & 2.220 \\
$\mathrm{Au}$ & $\mathrm{L}_{\alpha_{1}}$ & 9.175 \\
\hline
\end{tabular}

if there is excess oxygen in the particle impossible under these conditions.

Another X-ray technique may be more suitable to determining the chemical composition of the nanoparticles. X-Ray Photoelectron Spectroscopy (XPS) is capable of determining the oxidation state of an atom. Thus a tantalum-oxygen bond will produce a different peak than a tantalum-tantalum bond. An XPS experiment should be done in the future to understand the full chemical composition of the nanoparticles.

The end result of the EDX study shows clearly that the nanoparticles consist of tantalum, which is hardly surprising, but this shows conclusively that it is true. No other contaminant elements are observed, limiting the composition of the particles to either pure tantalum or some oxide form. Unfortunately, the exact composition with regards to oxygen could not be determined.

\section{Nanoparticle Deposition on Graphene Substrate}

In order to investigate the particle structure with the highest resolution possible, the tantalum nanoparticles were deposited on a graphene substrate. Free standing graphene, consisting of a single layer of carbon atoms, is the thinnest possible support, and therefore will produce the best possible contrast between the background and particles that the instrument is capable of.

A lacey carbon coated TEM grid was used as the support material for the chemical vapor deposition grown graphene. As the lacey carbon structure is full of holes but the graphene is a continuous film, there are many open regions of free standing graphene with no other contribution to the background. These regions should be ideal for obtaining the highest resolution.

To prove the existence of single layer of graphene, a diffraction pattern was taken in a region with a hole in the underlying lacey carbon structure. The resulting six-fold symmetry of the graphene hexagonal lattice can be seen in the right panel of Figure 10.35. 

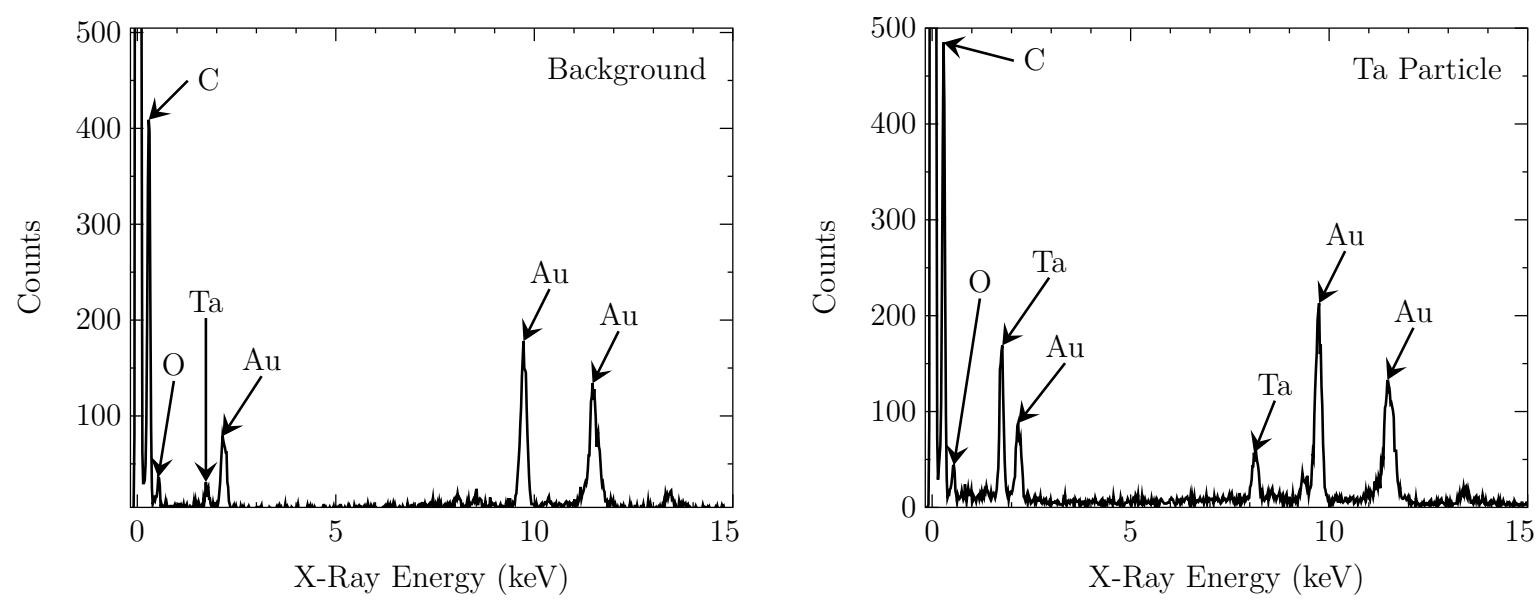

Figure 10.33: Energy Dispersive X-ray point scan spectra obtained from 300,000 amu tantalum nanoparticle on lacey carbon coated gold grid. Left: Point taken with no Ta particles. Right: Point taken on a Ta particle.

The underlying carbon film is amorphous and would appear as a diffuse ring if it were present, implying this region consists purely of single layer graphene.

$600,000 \mathrm{amu}$ tantalum nanoparticles deposited on this graphene support are shown in the left panel of Figure 10.35. This is an open graphene region - though a bit of the lacey carbon support film can be observed in the top portion of the image. The particle coverage is dense and the mass is rather high, but there does not seem to be substantial aggregation, compared to the previous results on the amorphous carbon film. This could imply that these nanoparticles are more stable on the single graphene layer, or more prone to binding to defects in the graphene. A further investigation of the stability of nanoparticles on graphene would be interesting for future experiments - especially given the scientific community's interest in the properties and applications of graphene.

The structure of the tantalum nanoparticles can be seen in the high magnification TEM in Figure 10.36. The resolution of this image should be high enough to see lattice fringes if the particles were crystalline. No structure can be seen, which implies that these particles are amorphous. This supports the findings in Reference [55], which also reports sputter deposited Ta observed in TEM as being amorphous. It may be that the particles are initially deposited with structure, but upon exposure to atmosphere they are oxidized to an amorphous structure of $\mathrm{TaO}_{x}$. One important characteristic to note is there is also no observation of a "core-shell" structure. An oxygen layer which only covers the surface would be expected to produce an observably different outer shell on the nanoparticle. The lack of this shell may suggest if the particle is oxidized then it is the entire particle, not just a thin surface layer. 
Chapter 10 Characterization and Preliminary Results
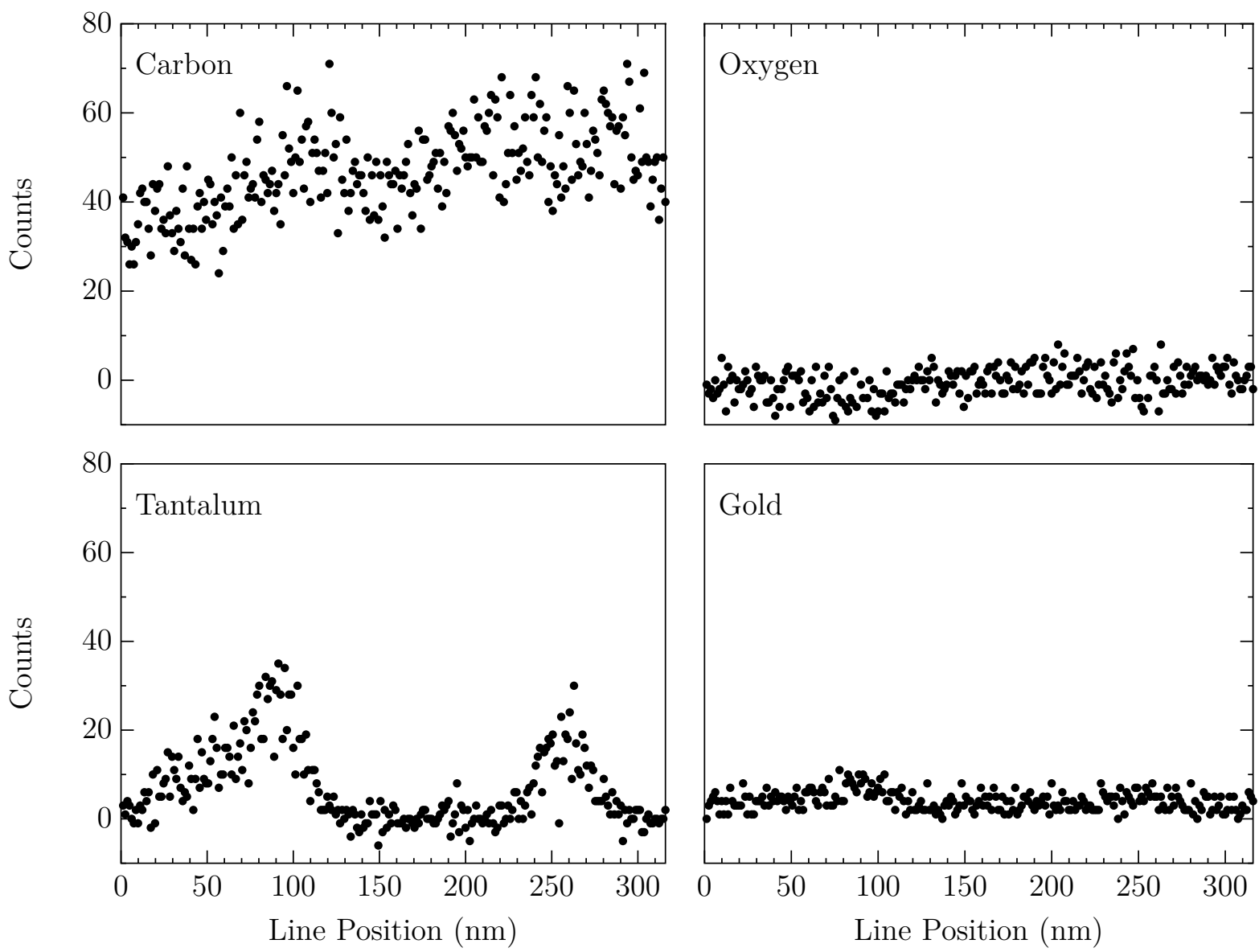

Figure 10.34: Energy Dispersive X-ray line scan spectra obtained from 300,000 amu tantalum nanoparticles on lacey carbon coated gold grid. Top Left: Carbon signal as function of line position. Top Right: Oxygen signal as function of line position. Bottom Left: Tantalum signal as function of line position. Bottom Right: Gold signal as function of line position. 

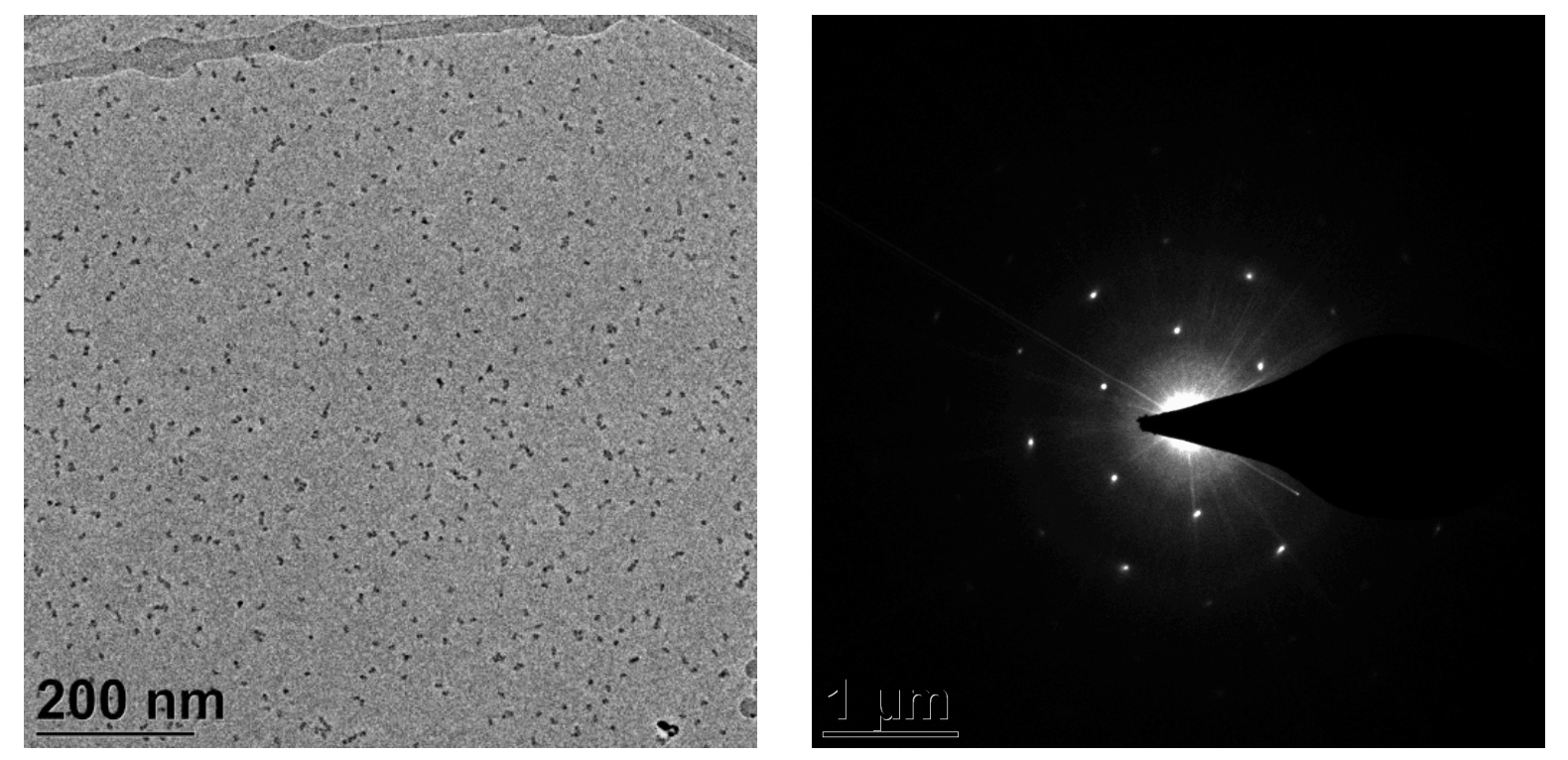

Figure 10.35: Left: Raw transmission electron microscope image of 600,000 amu tantalum nanoparticles deposited on a graphene coated TEM grid. Right: Image of diffraction pattern taken with the graphene coated substrate.

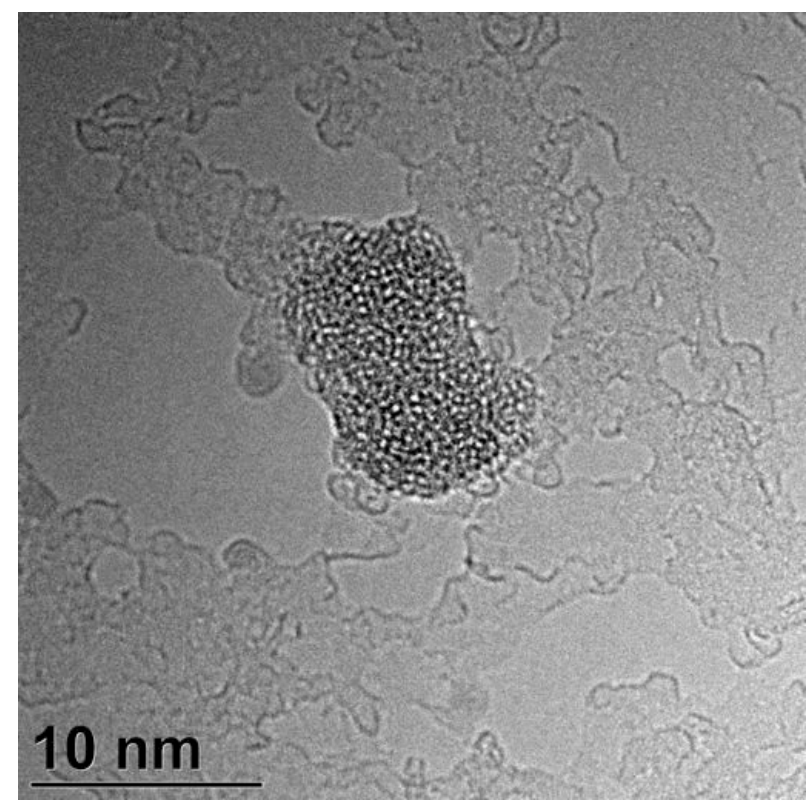

Figure 10.36: Raw transmission electron microscope image with high magnification of $600,000 \mathrm{amu}$ tantalum nanoparticles deposited on a graphene coated TEM grid. 


\section{Summary of TEM results}

The end result of the electron microscopy on the deposited nanoparticles may leave more questions than answers. An initial goal was to provide a size distribution which was less prone to unaccountable effects which are present in AFM. While a clear size determination could be made, it could only be done on a narrow range of masses which produced enough contrast and low aggregation. Outside this range, it seems clear the particle size is varying in a manner consistent with expectation - the very light particles are extremely small and the very heavy particles are larger. In the narrow range of quantification, the particle size scaled far beyond what the mass would predict. This implies particle growth due to oxidation is occurring. However, attempts at quantifying the oxidation through EDX were inconclusive at best. Finally, attempts at observing the particle's lattice structure through HR-TEM, which also could lead to a specific chemical assignment, showed the particles are amorphous.

This leaves several possible experiments open to future research. First, a higher resolution microscope and thinner substrates could allow for better images of smaller particles. Materials other than tantalum, which may be less prone to oxidation, could also be observed - perhaps displaying different aggregation kinetics. Particles which are stable to aggregation on the amorphous carbon film over a wider mass range would be a clear improvement for this type of study. A more conclusive composition analysis of the tantalum nanoparticles is desirable. X-Ray Photoelectron Spectroscopy (XPS) may offer the best chance at determining the presence and exact degree of oxidation of the nanoparticles. Further exploration of graphene as a substrate for nanoparticle deposition could be extremely interesting, especially in light of the observed improvement to the stability of nanoparticles with respect to aggregation at the higher masses. 


\section{Chapter 11}

\section{Summary and Outlook}

I have shown the full development, implementation and characterization of a new system for easily size-selecting and depositing nanoclusters on a variety of materials. Here I will summarize the essential points of the system, propose possible modifications and variations which could be implemented in the future and some proposed experiments which take advantage of this system's capabilities.

\subsection{Summary of Results}

The following achievements and milestones were accomplished in this dissertation work:

- The full assembly of a new instrument for the purpose of ion soft landing deposition of size selected nanoparticles.

- A characterization of the magnetron sputtering source, determining the effect the source parameters have on the nanoparticle growth and size distributions.

- A custom time of flight mass analyzer was designed, built, simulated, characterized and implemented in the system to directly measure the nanoparticle mass distribution.

- The quadrupole mass filter's performance for size selecting nanoparticles produced in the magnetron sputtering source was characterized.

- The kinetic energy distribution of the nanoparticle ion beam was measured under a variety of source conditions using a custom built retarding field energy analyzer.

- The deposited nanoparticles were characterized through two types of microscopy on a variety of substrates.

The overall stated goal of achieving soft landing of size selected nanoclusters on surfaces with this instrument was demonstrated and the instrument is fully capable of moving forward to the experiments for which it was initially proposed.

The nanoparticle size distribution and ion beam intensity was found to be dependent on the following adjustable parameters: 
Chapter 11 Summary and Outlook

- Sputtering Power

- Sputtering Gas (Argon) Flow

- Aggregation Gas (Helium) Flow

- Length of Aggregation Region

- Exit Aperture Size

The sputtering power was determined to increase the cluster size, the width of the size distribution and the intensity of the ion beam with increasing power. This parameter is one of the easiest to adjust with precise control to increase the ion current for a desired mass.

The sputtering gas flow was found to generally increases the average size and width of the cluster mass distribution up to a limit. Beyond this limit the cluster size tends to decrease again. This is an important parameter for adjusting the mass distribution to maximize intensity for a given mass, but the response to changes can be slow and unpredictable, and the control is not as precise as the sputtering power.

The aggregation gas flow was found to greatly improve the cluster intensity and decrease the size and width of clusters when the flow increases. This is likely due to the aggregation gas enabling more cluster seeds to form without providing additional free metal atoms from additional sputtering. Controlling the ratio of argon to helium is another powerful method of enhancing cluster growth at a desired size, but also difficult to predict exactly what ratio will produce the desired effect.

The aggregation region length was shown to generally increase the size of clusters with longer aggregation distance. This parameter is easily adjusted with fine control, though has little effect for small changes.

Finally, the exit aperture size was found to greatly affect the pressure inside the aggregation region. Large apertures reduce the interior pressure and greatly reduces the maximum size the clusters grow to. This could be one of the most powerful ways to influence the cluster size distribution, though unfortunately it cannot currently be adjusted without a full venting of the vacuum chamber as the apertures are fixed to the exit with screws.

A custom built time of flight mass spectrometer was designed to allow for independent characterization of the nanocluster mass distribution. A compact design was conceived, built and implemented in the nanoparticle deposition system. The instrument was calibrated over a wide mass range in the system using clusters of the single-isotope element tantalum. The experimental results were consistent with analytical ion simulations in SIMION and numerical calculations. The calibration was performed for several extraction pulse voltages. The instrument was shown to be functional for the characterization of the mass distribution of the nanoparticle ion beam.

The performance of the commercially bought quadrupole mass filter was independently characterized using the time of flight mass analyzer. The masses transmitted through the quadrupole were found to differ from the theoretically expected masses by $42 \%$ and the 
correction factor was applied to the instrument's electronics. The instrument was proven to be able to adjust the mass of the transmitted nanoparticles. The mass resolution was determined to behave within expectations given by quadrupole theory. Overall, the instrument was shown to function for its stated purpose and can be used as an effective means of size selecting nanoparticles.

Controlled deposition energy for the soft landing of nanoparticles is one of the stated objectives of this system. The kinetic energy distribution of the nanoparticle ion beam produced through magnetron sputtering needed to be measured and understood to achieve this objective. A home built retarding field energy analyzer was implemented into the system and the energy of the ion beam measured directly. The results show the nanoparticle's energy is mass dependent and best described by a "velocity slip" energy model common to the field of molecular beams. The nanoparticle energy is shown to be dependent on the source gas flow parameters. The nanoparticle energy was shown to be well below the energy threshold for soft-landing.

Size selected nanoparticles were deposited at soft landing energies on a variety of surfaces and examined by microscopy. The size distributions were shown to scale with the mass of the nanoparticle, but not exactly within the expected size from a spherical particle approximation. The results could not determine the exact cause of the deviation. Possible explanations include oxidation of the particles, non-spherical particles or poor assumptions in the expected size calculation. More experiments should be done in the future to investigate the role these effects play and improve the ability to predict the deposited size with selected deposition mass. In addition, the width of the size distribution of deposited particles is larger than would be expected from the mass resolution of the nanoparticle beam. Further investigation to improve the narrowness of the size distribution should be considered in the future.

Tantalum nanoparticles used in this characterization study were found to be remarkably thermally stable against aggregation kinetics on mica substrates. This result could provide an opportunity to study size selected tantalum nanoparticle catalysis under real world catalytic conditions.

\subsection{Experimental Outlook}

Completion of the assembly and characterization phase of this new system paves the way for work on new avenues of experimental research. Three clear proposals for impactful future experiments for this system have been made:

- Molecular Beam Scattering from Nanostructured Surfaces

- Quadrupole Ion Trapping of Nanoparticles

- Size Dependent Nanoparticle Catalysis

Experiments which investigate the interaction between vibrationally excited molecules and surfaces in a collision are on-going in the Surface Dynamics Group at Georg-August- 


\section{Chapter 11 Summary and Outlook}

Universität. The experiments thus far have concentrated on NO scattering on the model single crystal $\mathrm{Au}(111)$ surface. A development of a theoretical model to describe the observed interactions has been ongoing as well [7]. At some point, when the theory and experimental observations converge, new experimental results on more complex systems will be necessary to challenge the limits of the theory. This is where size selected nanoparticle surfaces could play an interesting role.

Plans are underway to develop another new instrument based on the current molecular beam scattering instrument [46] which would sit along side the size selected nanoparticle deposition system. This system would incorporate an in-vacuo sample transfer system to allow for the preparation of nanoparticle surfaces which could be used for molecular beam scattering experiments.

The current scattering experiments observe non-adiabatic coupling between the vibrational states of the NO molecule and the electronic states of the metallic $\mathrm{Au}(111)$ surface. No coupling is observed in collisions with insulating surfaces. The proposed experiments would observe what happens with this coupling as the surface transitions from the bulk metal surface to smaller and smaller nanoparticles. A further interesting topic that could be explored includes what role the particle size plays in dissociative adsorption of molecules, which is key to further understanding of nanoparticle catalysis size effects.

Control over the size distribution and particle density on surface are essential and discovery of ideal substrate materials to suppress aggregation kinetics will be important to developing this experiment. Of the three proposed experiments utilizing size selected nanoparticles, this one is the furthest away from being attempted.

In the mean time, a collaborative experiment has been developed with Dieter Gerlich, whose work in the field of ion traps is extensive. By installing a quadrupole ion trap after the quadrupole mass filter of the nanoparticle deposition system, the trap could be loaded with nanoparticles. Once trapped, these particles could remain in the trap for many hours or days. Optical access for lasers is available in this particular trap. Previous experiments by Dieter Gerlich and coworkers has observed black body radiation from $\mathrm{C}_{60}$ particles laser heated in this trap [49].

The proposed experiment would look for black body radiation being emitted from nanoparticles in the trap. This would allow the secular frequency of the trapped particles to be observed optically. As the frequency of the oscillating particle in the trap is related to its mass, this would be an optical method of mass spectrometry of nanoparticles. This could have important implications as this method could scale to mass and size ranges which are impractical for other forms of mass spectrometry.

The quadrupole ion trap is already in testing for use with the size selected nanoparticle deposition system and these experiments are ongoing.

Additionally, catalysis using nanoparticles is currently an extremely active area of research, following the discovery of enhanced catalytic activity of Au nanoparticles by Masutake Haruta [23-25]. The size of the nanoparticles used in catalysis plays a large role in their activity which opens the door for systematic experiments using the size selected nanoparticle deposition system developed in this dissertation. The system has the ability to prepare catalysts with any substrate and a narrow size distribution of any sputtering 
compatible material.

A reaction cell connected to an atmospheric pressure sampling mass spectrometer is currently being constructed to measure the catalytic activity of prepared nanoparticle samples. Catalysts prepared in the nanoparticle deposition system could be transferred to the reaction cell to determine their activity. While there are many research groups devoted to exploring nanoparticle size effects in catalysis, this project could have a significant advantage.

In the Fall of 2012, the Materials Physics department at Georg-August-Universität will have an FEI Titan Environmental Transmission Electron Microscope (ETEM) with aberration correction installed. This instrument will allow for high resolution TEM images with the specimen at elevated temperatures in a reactive gas with pressures up to several millibars. This would allow for real time observation of nanoparticle catalysts under reactive conditions. Collaboration with this instrument will allow for in-depth examination of the structure of reactive nanoparticle catalysts and sintering kinetics in catalyst samples.

The structure of this experiment would be to identify good catalytic materials and reactions exhibiting a significant dependence on size using the mass spectrometer reaction cell. Once interesting candidates are identified, the nanoparticle structure can be observed in the ETEM during the reaction. This experiment could greatly expand the understanding of how nanoparticle catalytic activity and size are related.

\subsection{Outlook for Instrument Modifications and Improvements}

While the current system is fully functional for the expected experiments in the near future, there are a number of areas where improvements could be made or current capabilities expanded upon.

The quadrupole mass filter has been shown to adequately perform the task of nanoparticle size selection. However, there is room for improvement. As demonstrated, the ion beam exiting the quadrupole is quite divergent which likely leads to unnecessary losses of otherwise usable ions. In addition, the mass resolution, while behaving as expected, leads to too low of an ion current at the best resolutions, making the best resolution of $2 \%$ unusable for deposition.

The quadrupole mass filter can theoretically have improved performance in these areas with the implementation of a collisional quadrupole between the source and filter. This is an RF-only quadrupole ion guide operated with a high pressure of rare gas inside. The ions experience a significant compression of phase space, particularly in the axial velocity. The net effect leads to a less divergent ion beam which may lead to higher ion transmission and be more suited to further focusing and manipulation through our ion optics. This would have the dual effect of improving ion current at highest resolution and forming a less divergent ion beam.

Improvements to the ion optics system could serve a similar purpose as the collisional 
quadrupole. Losses in the ion beam lead to some limitations on the usable range of filtered ions. By replacing the einzel lens with a multipole ion guide, the entire beam exiting the quadrupole could be guided directly to the deposition surface with minimal losses. Improvements in this area may require little investment but produce significant gains in the ion beam current.

The ion filtering and deposition systems are not directly tied to use of a magnetron sputtering ion source. While magnetron sputtering offers several advantages to the stated goals of this instrument, in theory, any ion source could be used for size selection and deposition. Several ideas have been proposed for future ion sources which could be swapped in place of the magnetron position and allow for an even larger variety of materials to be deposited.

There has been recent development on a flame nanoparticle source at the Open University of Israel [26]. This source produces clusters in a similar way to the magnetron with gas aggregation, but the initial source is combusting an organometallic precursor in a flame. The advantage that could be gained through this source is a facile method to producing highly oxidized clusters, due to the nature of the flame combustion source. An electrospray ionization source would allow for the deposition of organic and biological materials. Finally, another variation on magnetron sputtering using an RF power supply which allows for sputtering to occur on insulating materials, opening up another avenue for research. This could be implemented with the current instrumentation as it only involves replacing the DC power supply with an RF one. 


\section{Bibliography}

[1] A.I. Ayesh et al. "Experimental and simulational study of the operation conditions for a high transmission mass filter". In: Rev. Sci. Instrum. 78.5 (2007), p. 053906.

[2] S.H. Baker et al. "The construction of a gas aggregation source for the preparation of size-selected nanoscale transition metal clusters". In: Rev. Sci. Instrum. 71.8 (2000), pp. 3178-3183.

[3] R.T. Bayard and D. Alpert. "Extension of the Low Pressure Range of the Ionization Gauge". In: Rev. Sci. Instrum. 21 (1950), p. 571.

[4] B. Briehl and H.M. Urbassek. "Monte Carlo simulation of growth and decay processes in a cluster aggregation source". In: J. Vac. Sci. Technol., A 17 (1999), p. 256.

[5] L. de Broglie. In: Ann. de Physique 3 (1925), p. 22.

[6] J.B. Chen et al. "Morphological studies of nanostructures from directed cluster beam deposition". In: Eur. Phys. J. D 34.1-3 (2005), pp. 251-254.

[7] R. Cooper et al. "Multiquantum Vibrational Excitation of NO Scattered from Au(111): Quantitative Comparison of Benchmark Data to Ab Initio Theories of Nonadiabatic Molecule-Surface Interactions". In: Angew. Chem. Int. Ed. 51 (2012), pp. 4954-4958.

[8] D.A. Dahl. "SIMION for the personal computer in reflection". In: Int. J. Mass. Spectrom. 200 (2000), p. 3.

[9] P.H. Dawson. "Quadrupole mass analyzers: Performance, design and some recent applications". In: Mass Spectrom. Rev. 5.1 (1986), pp. 1-37.

[10] D.J. Douglas. "Linear quadrupoles in mass spectrometry". In: Mass Spectrom. Rev. 28.6 (2009), pp. 937-960.

[11] R.C. Flagan and M.M. Lunden. "Particle structure control in nanoparticle synthesis from the vapor phase". In: Mater. Sci. Eng., A 204.1-2 (1995), pp. 113-124.

[12] I.M. Goldby et al. "Gas condensation source for production and deposition of sizeselected metal clusters". In: Rev. Sci. Instrum. 68.9 (1997), pp. 3327-3334.

[13] A. V. Goncharov and P. V. Kashtanov. "Modeling of cluster formation and growth under atomic vapor condensation". In: High Temp. 49.2 (2011), pp. 178-186.

[14] C.G. Granqvist and R.A. Buhrman. "Ultrafine Metal Particles". In: J. Appl. Phys. 47.5 (1976), pp. 2200-2219.

[15] M. Guilhaus. "Principles and Instrumentation in Time-of-flight Mass Spectrometry". In: J. Mass Spec. 30 (1995), pp. 1519-1532. 
Bibliography

[16] Gwyddion 2.29 - Free SPM data analysis software, http://gwyddion.net.

[17] H. Haberland, Z. Insepov, and M. Moseler. "Molecular dynamics simulation of thin film formation by energetic cluster impact (ECI)". In: Z. Phys. D 26.1-4 (1993), pp. 229-231.

[18] H. Haberland, M. Karrais, and M. Mall. "A new type of cluster and cluster ion source". In: Z. Phys. D 20.1-4 (1991), pp. 413-415.

[19] H. Haberland et al. "Filling of micron-sized contact holes with copper by energetic cluster impact". In: J. Vac. Sci. Technol., A 12.5 (1994), pp. 2925-2930.

[20] H. Haberland et al. "Thin film growth by energetic cluster impact (ECI): comparison between experiment and molecular dynamics simulations". In: Mater. Sci. Eng., B 19.1-2 (1993), pp. 31-36.

[21] H. Haberland et al. "Thin films from energetic cluster impact: A feasibility study". In: J. Vac. Sci. Technol., A 10.5 (1992), pp. 3266-3271.

[22] W. Harbich. In: Metal Clusters at Surfaces. Ed. by K.H. Weiwes-Broer. Springer, 2000 .

[23] M. Haruta. "Catalysis of gold nanoparticles deposited on metal oxides". In: Cattech 6.3 (2002), pp. 102-115.

[24] M. Haruta. "Size- and support-dependency in the catalysis of gold". In: Catal. Today 36.1 (1997), pp. 153-166.

[25] M. Haruta and M. Date. "Advances in the catalysis of Au nanoparticles". In: Appl. Catal., A 222.1-2 (2001), pp. 427-437.

[26] A. Hevroni et al. "In situ measurement of the mass concentration of flame-synthesized nanoparticles using quartz-crystal microbalance". In: Meas. Sci. Technol. 22 (2011), p. 115102.

[27] Igor Pro 6.0, WaveMetrics, Inc., http://www.wavemetrics.com.

[28] P. Jensen. "Growth of nanostructures by cluster deposition: Experiments and simple models". In: Rev. Mod. Phys. 71.5 (1999), pp. 1695-1735.

[29] P.V. Kashtanov, B.M. Smirnov, and R. Hippler. "Efficiency of cluster generation in a magnetron discharge". In: Europhys. Lett. 91.6 (2010), p. 63001.

[30] P.V. Kashtanov, B.M. Smirnov, and R. Hippler. "Magnetron plasma and nanotechnology". In: Phys. Usp. 50.5 (2007), pp. 455-488.

[31] A. Kühle, A.H. Sørensen, and J. Bohr. "Role of attractive forces in tapping tip force microscopy". In: J. Appl. Phys. 81 (1997), p. 6562.

[32] LabVIEW, National Instruments, http://www.ni.com/labview/.

[33] G.A. Lukyanov, L.Y. Nikolaeva, and N.Y. Bykov. "Direct Simulation Monte-Carlo of Clusters Formation Process in Magnetron-type Generator". In: Fullerenes, Nanotubes and Carbon Nanostructures 16 (2008), pp. 551-554. 
[34] A. Majumdar et al. "Development of metal nanocluster ion source based on dc magnetron plasma sputtering at room temperature". In: Rev. Sci. Instrum. 80.9 (2009), p. 095103.

[35] E. Mathieu. "Mémoire sur Le Mouvement Vibratoire d'une membrane de forme elliptique". In: Journal de Mathématiques Pures et Appliqúees (1868), pp. 137-203.

[36] D. Mott et al. "Size Determination of Nanoparticles Based on Tapping-Mode Atomic Force Microscopy Measurement". In: Journal of Scanning Probe Microscopy 3 (2008), pp. 1-8.

[37] A.M. Myers et al. "Monte Carlo simulations of magnetron sputtering particle transport". In: J. Vac. Sci. Technol., A 9.3 (1991), pp. 614-618.

[38] P. Nemes-Incze et al. "Anomalies in thickness measurements of graphene and few layer graphite crystals by tapping mode atomic force microscopy". In: Carbon 46 (2008), pp. 1435-1442.

[39] S.J.T. Van Noort et al. "Height anomalies in tapping mode atomic force microscopy in air caused by adheasion". In: Ultramicroscopy 69 (1997), pp. 117-127.

[40] D.L. Olynick, J.M. Gibson, and R.S. Averback. "Impurity-suppressed sintering in copper nanophase materials". In: Phil. Mag. A 77.5 (1998), pp. 1205-1221.

[41] W. Paul. "Electromagnetic traps for charge and neutral particles". In: Rev. Mod. Phys. 62.3 (1990), pp. 531-540.

[42] W. Paul, H.P. Reinhard, and U. Vonzahn. "Das elektrische Massenfilter als Massenspektrometer und Isotopentrenner". In: Z. Phys. 152.2 (1958), pp. 143-182.

[43] F.M. Penning. "Über Ionization durch metastabile Atome". In: Naturwissenschaften 15 (1927), p. 818.

[44] S. Pratontep et al. "Size-selected cluster beam source based on radio frequency magnetron plasma sputtering and gas condensation". In: Rev. Sci. Instrum. 76.4 (2005), p. 045103.

[45] QMF200 Quadrupole Mass Filter, Manual, Ver. 1.1. Oxford Applied Research Ltd.

[46] Q. Ran et al. "An advanced molecule-surface scattering instrument for study of vibrational energy transfer in gas-solid collisions". In: Rev. Sci. Instrum. 78 (2007), p. 104104.

[47] W.S. Rasband. ImageJ, U.S. National Institutes of Health, Bethesda, Maryland, USA, http://imagej.nih.gov/ij. 1997-2012.

[48] R. Reichel et al. "Construction and application of a UHV compatible cluster deposition system". In: J. Nanopart. Res. 8.3-4 (2006), pp. 405-416.

[49] S. Schlemmer et al. "Interaction of electrons and molecules with a single trapped nanoparticle". In: Appl. Phys. A 78 (2004), pp. 629-636.

[50] I. Shyjumon et al. "Deposition of titanium/titanium oxide clusters produced by magnetron sputtering". In: Thin Solid Films 500.1-2 (2006), pp. 41-51. 
Bibliography

[51] I. Shyjumon et al. "Structural deformation, melting point and lattice parameter studies of size selected silver clusters". In: Eur. Phys. J. D 37.3 (2006), pp. 409-415.

[52] V. Straňák et al. "Size-controlled formation of $\mathrm{Cu}$ nanoclusters in pulsed magnetron sputtering system". In: Surf. Coat. Technol. 205.8-9 (2011), pp. 2755-2762.

[53] Tables of Physical and Chemical Constants (16th Edition 1995). 4.2.1 X-Ray Absorption edges, characteristic X-Ray lines and fluorescence yields. Kaye 86 Laby Online. Version 1.0. www.kayelaby.npl.co.uk. 2005.

[54] M. Tello et al. "Imaging cobalt nanoparticles by amplitude modulation atomic force microscopy: comparison between low and high amplitude solutions". In: Ultramicroscopy 97 (2003), pp. 171-175.

[55] F.K. Urban et al. "Nanophase films deposited from a high-rate, nanoparticle beam". In: J. Vac. Sci. Technol., B 20.3 (2002), pp. 995-999.

[56] M. Di Vece, S. Palomba, and R.E. Palmer. "Pinning of size-selected gold and nickel nanoclusters on graphite". In: Phys. Rev. B 72.7 (2005), p. 4.

[57] W.C. Wiley and I.H. McLaren. "Time-of-Flight Mass Spectrometer with Improved Resolution". In: Rev. Sci. Instrum. 26 (1955), pp. 1150-1157.

[58] D.B. Williams and C.B. Carter. Transmission Electron Microscopy: A textbook for materials science. 1st Edition. Springer, 2004.

[59] L. Zitzler, S. Herminghaus, and F. Mugele. "Capillary forces in tapping mode atomic force microscopy". In: Phys. Rev. B 66 (2002), p. 155436. 


\section{Appendix A}

\section{Quadrupole Mass Filter Calibration Data}

A table containing all of the data points used in calibrating the quadrupole mass filter in Chapter 10.3.2 along with each point's individual $k$ value. The $k$ value determined from the best fit line through all points was 1.42 .

Table A.1: Quadrupole mass filter calibration data

\begin{tabular}{cccc}
\hline $\begin{array}{c}\text { Selected Mass } \\
(\mathrm{amu})\end{array}$ & $\begin{array}{c}\text { Fit Peak Time } \\
(\text { psec })\end{array}$ & $\begin{array}{c}\text { Calculated Mass } \\
(\mathrm{amu})\end{array}$ & $k$ value \\
\hline 30,037 & 58.148 & 41,347 & 1.38 \\
40,019 & 68.052 & 56,669 & 1.42 \\
50,001 & 76.471 & 71,589 & 1.43 \\
60,029 & 83.996 & 86,398 & 1.44 \\
70,011 & 90.392 & 100,080 & 1.43 \\
80,085 & 96.418 & 113,889 & 1.42 \\
90,113 & 102.13 & 127,803 & 1.42 \\
100,094 & 107.53 & 141,694 & 1.41 \\
110,076 & 112.68 & 155,609 & 1.41 \\
120,104 & 117.66 & 169,684 & 1.41 \\
130,132 & 122.42 & 183,708 & 1.41 \\
140,114 & 126.93 & 197,508 & 1.41 \\
150,142 & 131.34 & 211,486 & 1.41 \\
160,170 & 135.7 & 225,775 & 1.41 \\
170,198 & 139.98 & 240,256 & 1.41 \\
180,180 & 144.13 & 254,727 & 1.41 \\
190,173 & 148.6 & 270,786 & 1.42 \\
200,108 & 152.36 & 284,676 & 1.42 \\
210,111 & 156.07 & 298,721 & 1.42 \\
220,113 & 159.52 & 312,086 & 1.42 \\
230,115 & 163.08 & 326,183 & 1.42 \\
240,117 & 166.64 & 340,591 & 1.42 \\
\hline
\end{tabular}

(continued on next page...) 
Appendix A Quadrupole Mass Filter Calibration Data

\begin{tabular}{|c|c|c|c|}
\hline $\begin{array}{l}\text { Selected Mass } \\
(\text { amu })\end{array}$ & $\begin{array}{c}\text { Fit Peak Time } \\
(\mu \mathrm{sec})\end{array}$ & $\begin{array}{l}\text { Calculated Mass } \\
(\text { (amu) }\end{array}$ & $\mathrm{k}$ value \\
\hline 250,119 & 169.95 & 354,267 & 1.42 \\
\hline 260,121 & 173.24 & 368,127 & 1.42 \\
\hline 270,123 & 176.53 & 382,253 & 1.42 \\
\hline 280,068 & 180.36 & 399,032 & 1.42 \\
\hline 290,107 & 183.66 & 413,779 & 1.43 \\
\hline 300,146 & 186.85 & 428,288 & 1.43 \\
\hline 310,186 & 189.99 & 442,815 & 1.43 \\
\hline 320,122 & 193.02 & 457,061 & 1.43 \\
\hline 330,161 & 196.12 & 471,871 & 1.43 \\
\hline 340,097 & 198.43 & 483,060 & 1.42 \\
\hline 350,033 & 201.26 & 496,946 & 1.42 \\
\hline 360,072 & 204.72 & 514,192 & 1.43 \\
\hline 370,112 & 207.76 & 529,586 & 1.43 \\
\hline 380,151 & 210.27 & 542,468 & 1.43 \\
\hline 390,190 & 213.13 & 557,335 & 1.43 \\
\hline 400,126 & 215.71 & 570,918 & 1.43 \\
\hline 410,166 & 218.68 & 586,758 & 1.43 \\
\hline 420,102 & 221.23 & 600,530 & 1.43 \\
\hline 430,187 & 222.97 & 610,019 & 1.42 \\
\hline 450,243 & 228.67 & 641,626 & 1.43 \\
\hline 470,115 & 233.58 & 669,493 & 1.42 \\
\hline 490,170 & 238.55 & 698,303 & 1.42 \\
\hline 510,042 & 243.31 & 726,464 & 1.42 \\
\hline 530,282 & 248.08 & 755,244 & 1.42 \\
\hline 550,154 & 252.65 & 783,341 & 1.42 \\
\hline 570,210 & 257.16 & 811,572 & 1.42 \\
\hline 590,449 & 261.72 & 840,624 & 1.42 \\
\hline 610,321 & 266.13 & 869,207 & 1.42 \\
\hline 630,193 & 270.33 & 896,872 & 1.42 \\
\hline 650,065 & 274.6 & 925,443 & 1.42 \\
\hline 670,305 & 278.8 & 953,983 & 1.42 \\
\hline 690,176 & 282.93 & 982,470 & 1.42 \\
\hline 740,224 & 293.32 & $1,055,988$ & 1.43 \\
\hline 790,317 & 300.59 & $1,109,007$ & 1.40 \\
\hline 840,411 & 310.4 & $1,182,608$ & 1.41 \\
\hline
\end{tabular}




\section{Appendix B}

\section{SIMION Geometry and Lua Program Files}

The geometry file representing the time of flight mass analyzer which was used to generate the SIMION simulation data in Chapters 9 and 10 is given here along with the lua programming file needed to produce the time dependent switch of the pulse voltage during the ion flight in SIMION.

\section{B.0.1 Geometry}

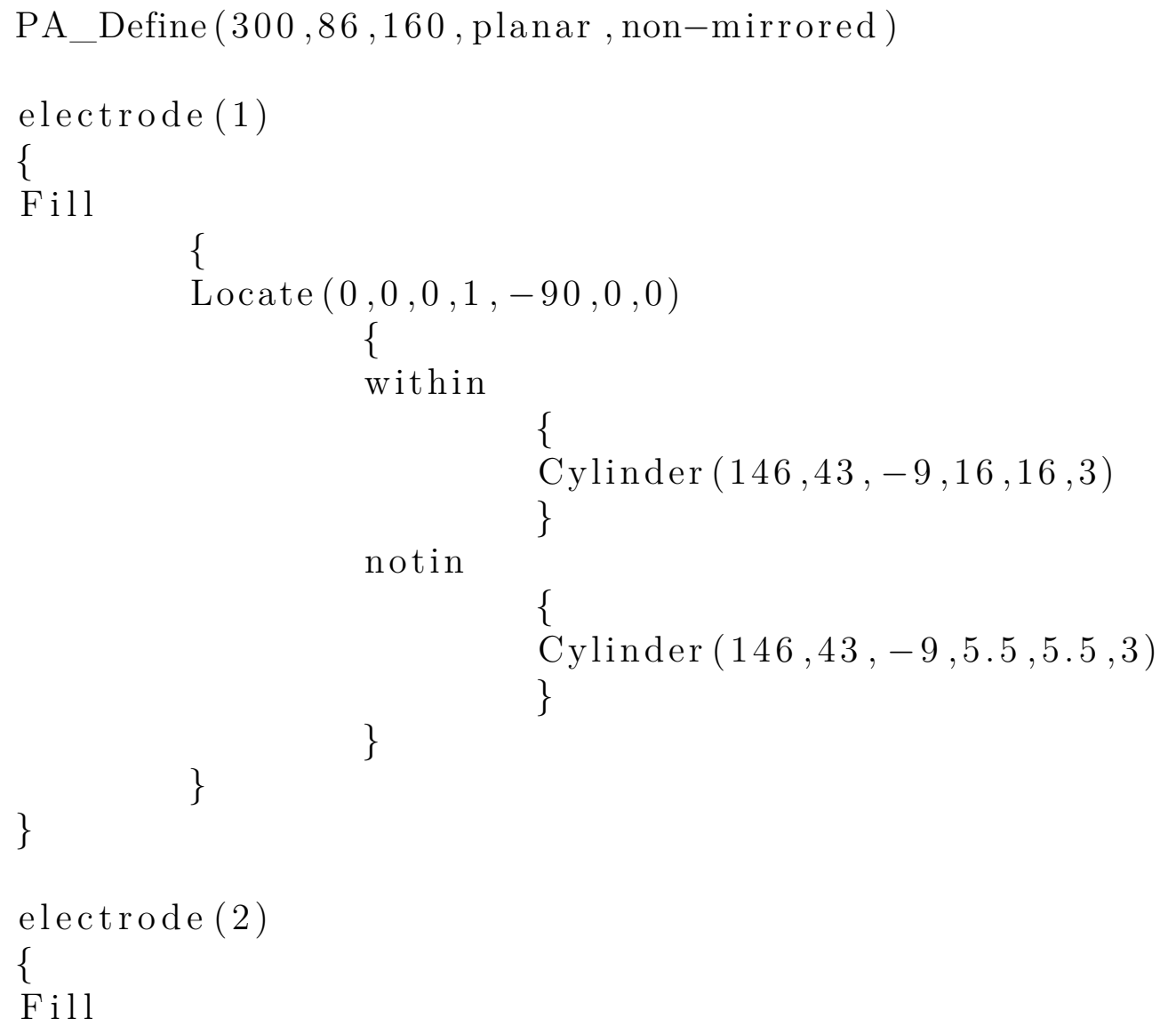


Appendix B SIMION Geometry and Lua Program Files

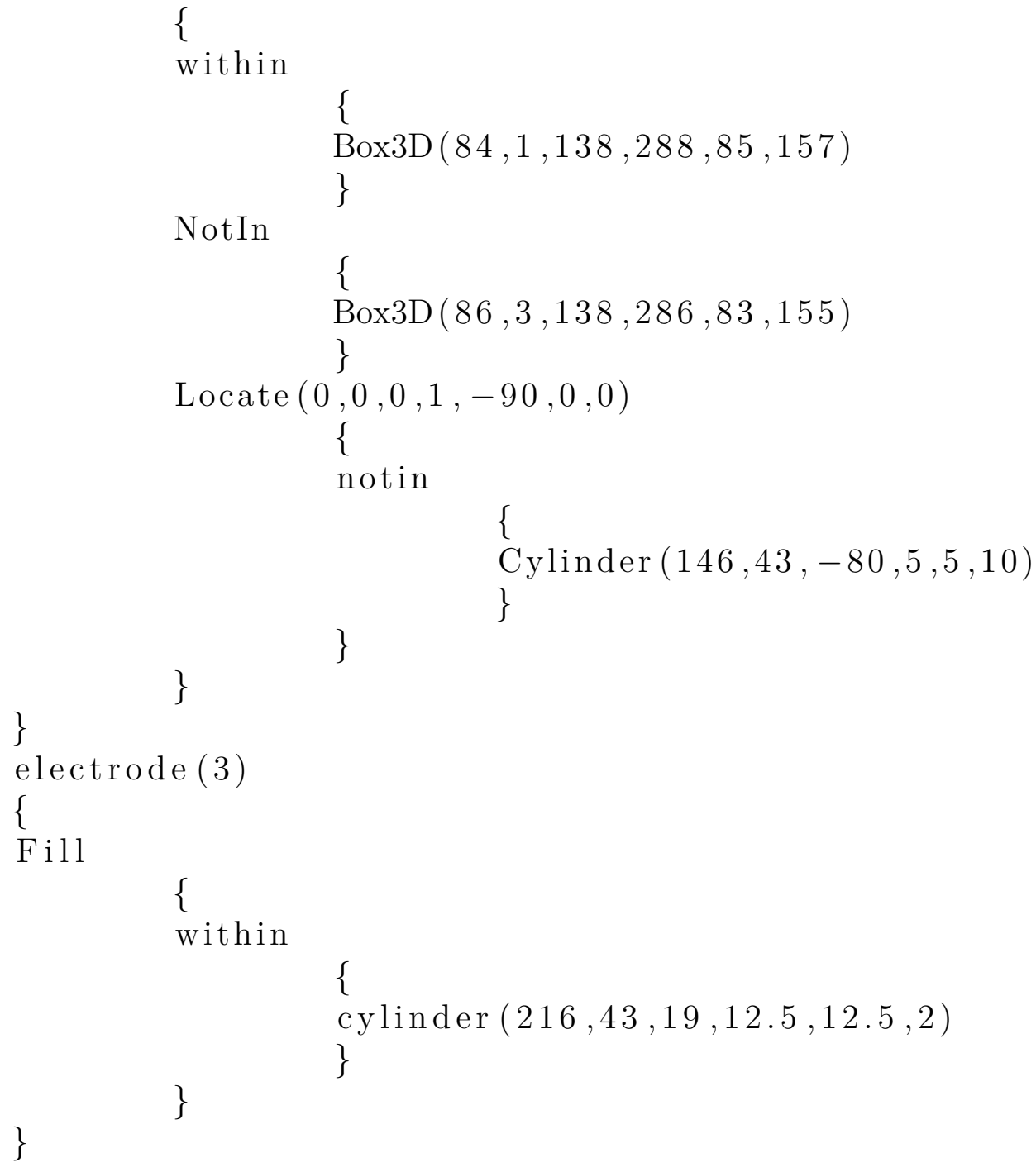




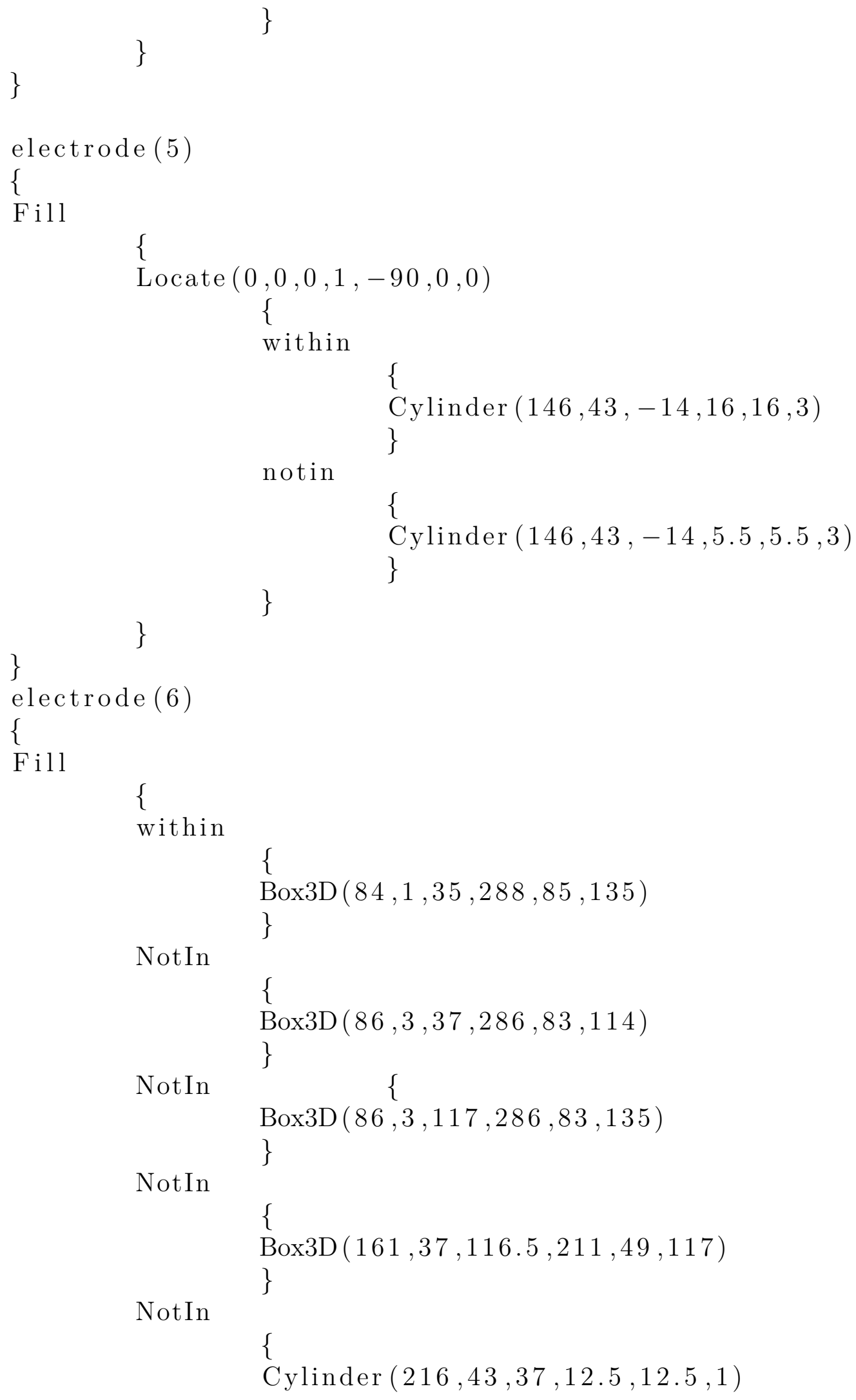


Appendix B SIMION Geometry and Lua Program Files

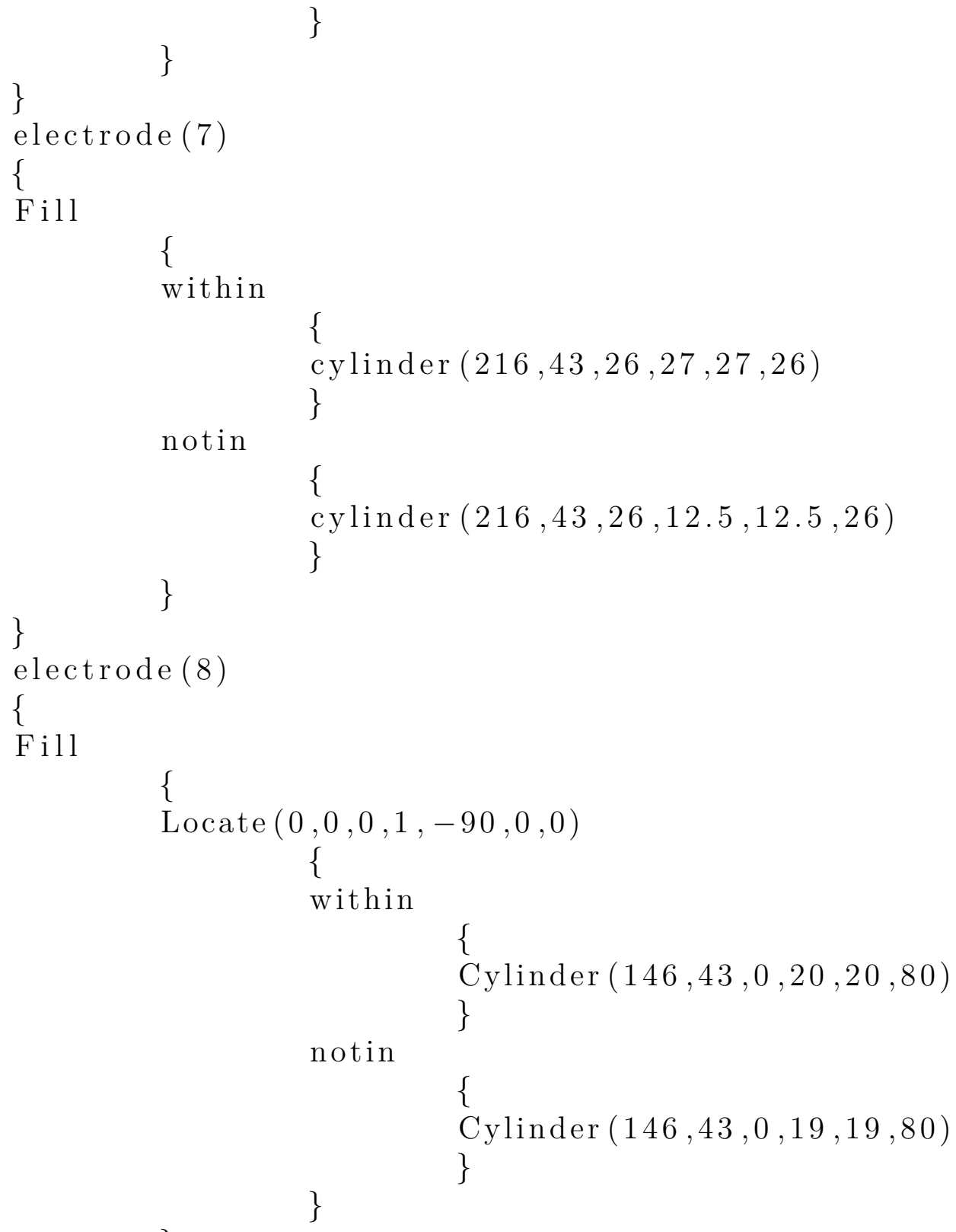

\section{B.0.2 Lua Program}

simion.workbench_program ()

adjustable pulse_voltage $=1000$

adjustable focus_voltage $=0$

adjustable detector_voltage $=8000$

adjustable p_on $=100$ 
adjustable p_duration $=200$

$$
\begin{aligned}
\text { function segment_init_p__values }() \\
\text { adj_elect } 01=\text { focus_voltage } \\
\text { adj_elect } 02=0 \\
\text { adj_elect } 03=\text { detector_voltage } \\
\text { adj_elect } 04=0 \\
\text { adj_elect } 06=0 \\
\text { adj_elect } 07=0
\end{aligned}
$$

end

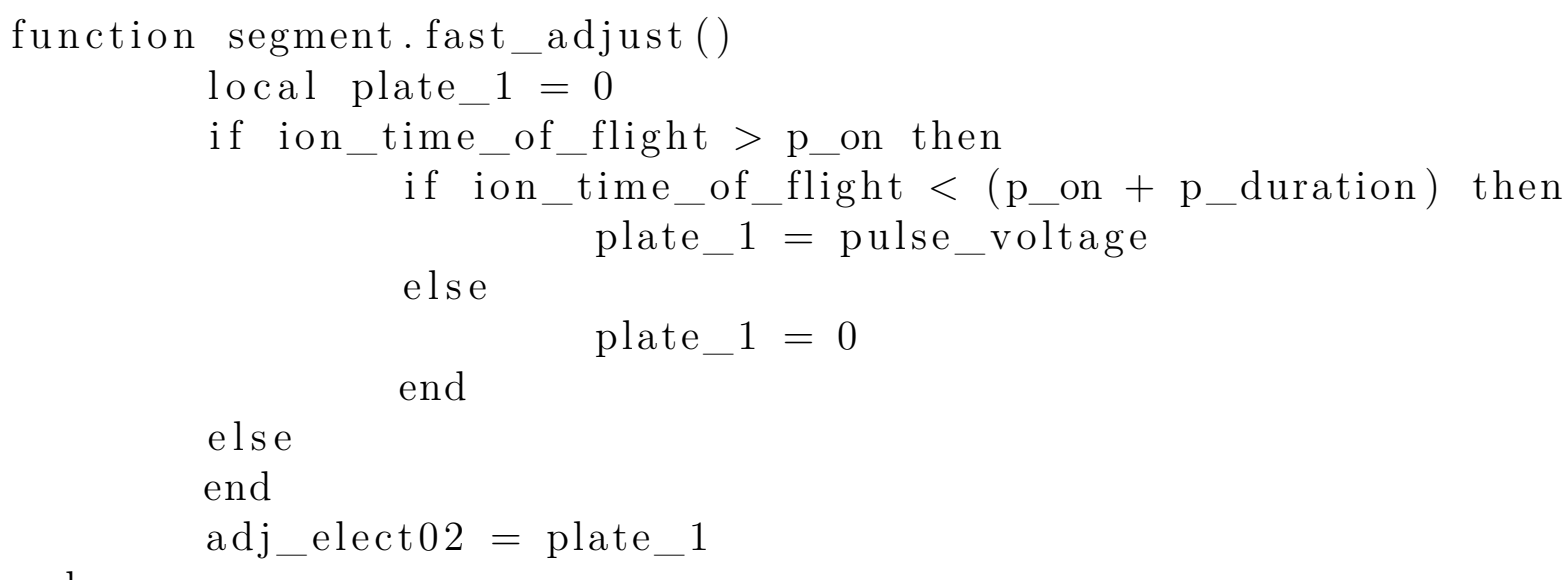


Appendix B SIMION Geometry and Lua Program Files 


\section{Acknowledgements}

First, I have to thank all of the members of the Wodtke group I have been fortunate enough to know and work with these last seven years, both in Santa Barbara and in Göttingen. This group has had the most diverse and talented people working in it and I can't imagine being anywhere else. I will miss you all. In particular I'd like to acknowledge the postdocs I have been fortunate enough to work with and who are my scientific role models. First, there are Petros Samartzis and Daniel Matsiev who introduced me to the wonders of coffee. Aside from being some of the most caffeinated people I know, they seem to have boundless knowledge and talking things through with them was always enlightening. Myung Hwa Kim was a whirlwind in the lab and always kept me on my toes. Christof Bartels is one of the best scientists I have been fortunate enough to work with and has helped me immensely in transitioning to life in Germany. I would also like to mention Valérie Blanchet. Though we only worked together a brief time, I always kept her advice for success in science in mind.

Of course, one person was responsible for bringing all these people together and giving me the opportunity to know them. Alec will always have my deep and unending gratitude for taking a chance with me. I know of no other professor who would be so willing to take a bachelor student whose project was mostly a failure and not only accept them into their group for a $\mathrm{PhD}$, but also send them to Taiwan to conduct a research experiment using preciously limited time at a synchrotron facility without supervision.

I'd also like to mention some of my other teachers who were particularly impactful on my scientific development. Sharon Veenhof was my high school chemistry teacher and without her encouragement, I'm certain I would never have gone as far as I have. Leroy Laverman was my undergraduate advisor and is the one mostly responsible for my interest in physical chemistry and for suggesting I work with Alec.

None of this would be possible without the support of my family. My Mom and Dad have had to sacrifice endlessly for me throughout the years and continue to do so even now so that I could follow my dream all the way to Germany. Without their support, none of this would have been possible.

Finally, I would like to thank my loving partner Ryan. He has had to endure a lot from me over the years. Being pulled away from a comfortable life in Santa Barbara to live without a job in a crummy apartment in a foreign country is more of a sacrifice then I would want anyone to make for me. Without him I don't think I could have survived these past two years away from all our friends and family. I don't think there is any way for me to match the support you have given me, but I will spend the rest of my life trying to anyway. 


\section{Life Run}

\section{General Information}

Name: $\quad$ Christopher Scott Larson

Date of Birth: August 26, 1983

Birthplace: $\quad$ Los Angeles, California, USA

Nationality: American

\section{Education}

1991-1995: Dixie Canyon Elementary School

1995-1998: St. Francis De Sales School

1998-2001: Bishop Alemany High School

\section{Higher Education}

2001-2005: Undergraduate studies in the College of Creative Studies at the University of California, Santa Barbara

06/2005: Awarded Bachelor of Science in Creative Studies, Emphasis: Chemistry

2005-2010: Graduate studies with Prof. Alec Wodtke at the University of California, Santa Barbara

10/2010: Transfer of graduate studies from UCSB to Georg-August-Universität Göttingen

2010-2012: Completion of dissertation with Prof. Alec Wodkte at Georg-August-Universität Göttingen

11/2012: Dissertation Defense 